\title{
Pore-spanning membranes - a versatile tool to analyze SNARE-mediated single vesicle fusion
}

\section{Dissertation}

\author{
for the award of the degree \\ Doctor rerum naturalium \\ within the doctoral program \\ Physics of Biological and Complex Systems \\ of the Georg-August-University School of Science (GAUSS)
}

submitted by

\section{Raphael Hubrich}

from Bremen

Göttingen 2018 



\section{Members of the thesis advisory committee}

Prof. Dr. Claudia Steinem

Institut für Organische und Biomolekulare Chemie

Georg-August-Universität Göttingen

Prof. Dr. Reinhard Jahn

Max-Planck-Institut für Biophysikalische Chemie Göttingen

Prof. Dr. Ulf Diederichsen

Institut für Organische und Biomolekulare Chemie

Georg-August-Universität Göttingen

\section{Members of the examination board}

$1^{\text {st }}$ Referee: Prof. Dr. Claudia Steinem

Institut für Organische und Biomolekulare Chemie

Georg-August-Universität Göttingen

$2^{\text {nd }}$ Referee: Prof. Dr. Reinhard Jahn

Max-Planck-Institut für Biophysikalische Chemie Göttingen

\section{Further members of the examination board}

Prof. Dr. Silvio Rizzoli

Institut für Neuro- und Sinnesphysiologie

Universitätsmedizin Göttingen

Dr. Sebastian Kruss

Institut für Physikalische Chemie

Georg-August-Universität Göttingen

Prof. Dr. Michael Meinecke

Institut für Zelluläre Biochemie

Universitätsmedizin Göttingen

\section{Date of oral examination}

29.03.2018 



\section{Declaration}

I, Raphael Hubrich, hereby certify that my doctoral thesis entitled "Pore-spanning membranes

- a versatile tool to analyze SNARE-mediated single vesicle fusion" has been written independently and with no other sources and aids than quoted.

Göttingen, 2018

Raphael Hubrich 

Abstract Signal transmission in neurons and hormone secretion in neuroendocrine cells such as chromaffin cells are fundamental biological processes including SNARE- (soluble N-ethylmaleimidesensitive-factor attachment receptor) mediated membrane fusion as the key step of exocytosis. The exact mechanism of this process, and in particular how a transient rise of the intracellular $\mathrm{Ca}^{2+}$-concentration triggers or even accelerates fusion remains unclear until today.

In this work, an established in vitro fusion assay based on planar pore-spanning membranes (PSMs) was employed to analyze SNARE-mediated single vesicle fusion. The investigation focused on the impact of $\mathrm{PI}(4,5) \mathrm{P}_{2}$ and synaptotagmin-1 (syt-1) on SNARE-mediated fusion. It was found that a $\mathrm{PI}(4,5) \mathrm{P}_{2}$ content of 2 mol\% in the t-SNAREs (syx-1A, SNAP25) harboring PSM resulted in a remarkable fusion efficiency of $\sim 92 \%$ of the detected v-SNARE (syb 2) containing vesicles. Moreover, the coreconstitution of the $\mathrm{Ca}^{2+}$-sensor syt-1 into the vesicular membrane aside syb 2 resulted in a significant increase in fusion kinetics in the presence of $100 \mu \mathrm{M} \mathrm{Ca}^{2+}$. This was concluded from the finding that, under equal conditions, the docking lifetime in the absence of syt-1 was determined to be twice as long (+syt-1: $\tau_{\text {docking }}^{\text {max }}=22 \pm 2 \mathrm{~s} v s .-$ syt-1: $\tau_{\text {docking }}^{\max }=44 \pm 1 \mathrm{~s}$ ).

Aside from synthetic vesicle fusion, SNARE-mediated fusion of natural dense core vesicles, the chromaffin granules (CGs), was investigated. CGs were isolated from bovine glands to monitor and analyze single CG fusion behavior on artificial PSMs. Interestingly, CGs showed similar fusion kinetics on PSMs as observed for synthetic vesicles. This was revealed by highly comparable distributions of the docking lifetime. However, after the onset of fusion CGs exhibited a much more diverse fusion behavior in comparison to that of synthetic vesicles.

Moreover, a detailed mobility analysis of SNARE-bound CGs on PSMs was performed. CGs were found to be mobile on both parts of the PSM, the free-standing (f-PSM) and the solid supported one (s-PSM), with mean diffusion coefficients of $0.34 \mu \mathrm{m}^{2} / \mathrm{s}$ (f-PSM) and $0.12 \mu \mathrm{m}^{2} / \mathrm{s}$ (s-PSM). The model system of PSMs therefore constitutes the first planar artificial membrane on which mobility of SNARE-bound CGs was monitored and analyzed. In general, a very diverse diffusion behavior of CGs on PSMs was observed, which is in good agreement with that monitored in live chromaffin cells. 
Zusammenfassung Sowohl in der neuronalen Reizweiterleitung als auch bei der Sekretion von Hormonen in neuroendokrinen Zellen, wie den chromaffinen Zellen, stellt die SNARE- (soluble $\mathrm{N}$ ethylmaleimide-sensitive-factor attachment receptor) vermittelte Membranfusion den Schlüsselschritt der Exocytose dar. Der exakte Ablauf dieses Prozesses und insbesondere die Art und Weise, wie eine transiente Erhöhung der intrazellulären $\mathrm{Ca}^{2+}$-Konzentration den Prozess auslöst bzw. beschleunigt, konnte bis heute nicht endgültig aufgeklärt werden.

Im Rahmen dieser Arbeit wurde ein auf dem Modellsystem der porenüberspannenden Membran (PSM) basierender in vitro Fusionsassay zur Detektion und Analyse SNARE-vermittelter Einzelfusionsereignisse von Proteoliposomen eingesetzt. Der Fokus lag hierbei auf der Untersuchung des Einflusses von PI(4,5) $\mathrm{P}_{2}$ und Synaptotagmin-1 (syt-1) auf die SNARE-vermittelte Membranfusion. Es konnte gezeigt werden, dass ein Anteil von 2 mol\% des polyanionischen Phospholipids PI(4,5) $\mathrm{P}_{2}$ in der PSM zu einer sehr hohen Fusionseffizienz von $~ 92 \%$ führte. Darüber hinaus konnte in Anwesenheit des $\mathrm{Ca}^{2+}$-Sensors syt-1 in der Membran der Proteoliposomen bei einer eingestellten $\mathrm{Ca}^{2+}$-Konzentration von $100 \mu \mathrm{M}$ eine signifikante Steigerung der Fusionskinetik registriert werden. Diese Erkenntnis spiegelt die Tatsache wider, dass die beobachtete mittlere Lebensdauer eines gedockten Proteoliposoms vor dem Einsetzen der Fusion in der Gegenwart von syt-1 nur halb so lang war wie in dessen Abwesenheit (+syt-1: $\tau_{\text {docking }}^{\max }=22 \pm 2 \mathrm{~s} v s .-$ syt-1: $\tau_{\text {docking }}^{\max }=44 \pm 1 \mathrm{~s}$ ).

Neben dem Fusionsverhalten synthetischer Proteoliposomen wurde auch das von natürlichen sekretorischen Vesikeln, den chromaffinen Granula (CGs) analysiert. Diese wurden hierzu aus Rindernebennieren isoliert und ihr Einzelfusionsverhalten auf PSM untersucht. Interessanterweise zeigten diese natürlichen Vesikel eine vergleichbare Fusionskinetik wie synthetische Proteoliposomen, was aus der sehr ähnlichen Verteilung der ermittelten Lebensdauer des gedockten Zustandes gefolgert wurde. Nach dem Einsetzen der Fusion jedoch, angezeigt durch Lipidvermischung zwischen Vesikelmembran und PSM, offenbarten die CGs ein deutlich vielfältigeres Fusionsverhalten als die synthetischen Proteoliposomen.

Des Weiteren wurde die Mobilität SNARE-gebundener CGs auf PSMs genauer untersucht. CG Diffusion wurde hierbei auf beiden Bereichen der PSM, dem festkörperunterstützten (s-PSM) sowie dem freitragenden (f-PSM) Bereich detektiert und mittlere Diffusionskoeffizienten von $0.12 \mu \mathrm{m}^{2} / \mathrm{s}$ (s-PSM) und $0.34 \mu \mathrm{m}^{2} / \mathrm{s}$ (f-PSM) ermittelt. Das Modellsystem der PSM stellt somit die erste planare, artifizielle Membran dar, auf der CG Mobilität beobachtet und analysiert werden konnte. Insgesamt wurde ein sehr vielfältiges Diffusionsverhalten der CGs auf PSMs beobachtet, welches sehr gut mit jenem übereinstimmt, das in lebenden chromaffinen Zellen detektiert wurde. 


\section{Contents}

1 Introduction ...............................................................................................................1

1.1 SNARE-mediated membrane fusion in neuronal signal transmission........................

1.2 In vitro reconstitution of SNARE-mediated fusion .............................................

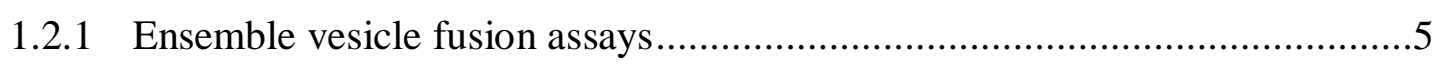

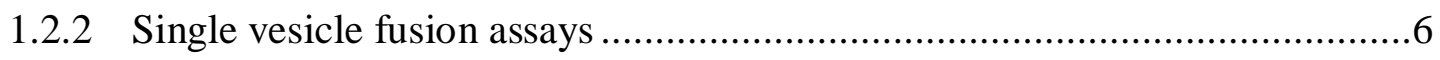

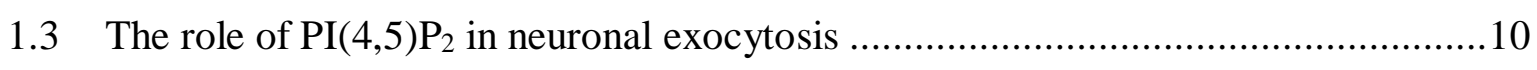

1.4 The role of synaptotagmin-1 in $\mathrm{Ca}^{2+}$-evoked exocytosis ......................................12

1.5 Chromaffin granules and SNARE-mediated exocytosis......................................15

2 Scope......................................................................................................................................17

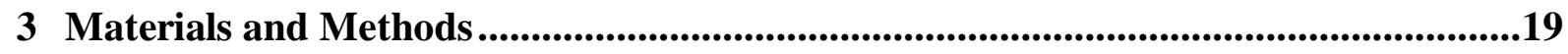

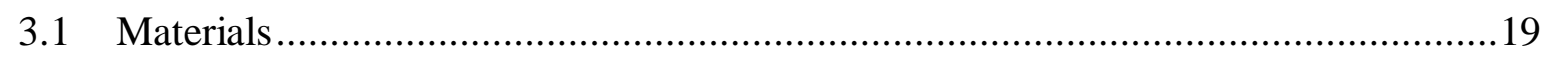

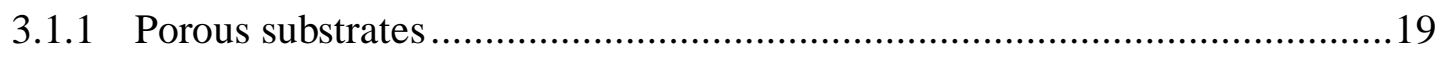

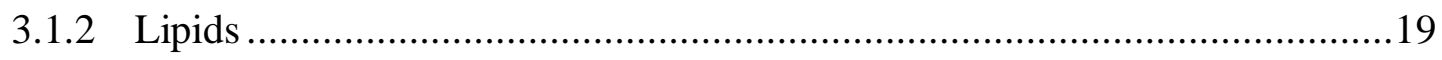

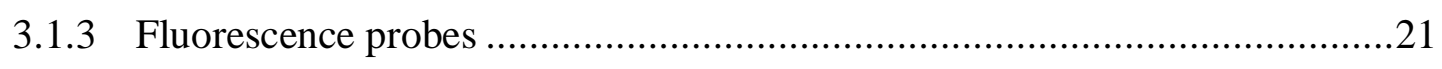

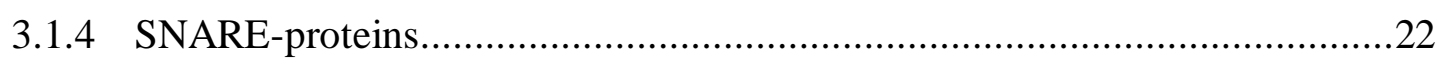

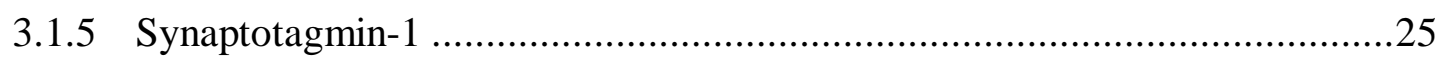

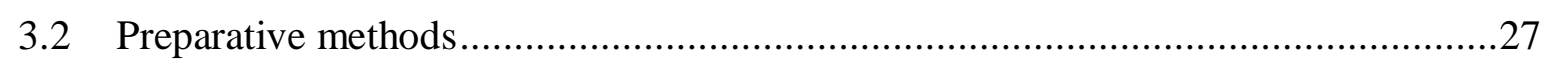

3.2.1 SDS-polyacrylamide gel electrophoresis (SDS-PAGE) ...........................27

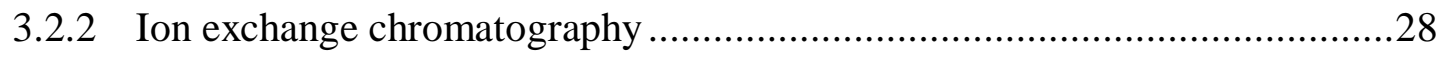

3.2.3 Isolation and purification of SNARE-proteins.......................................29

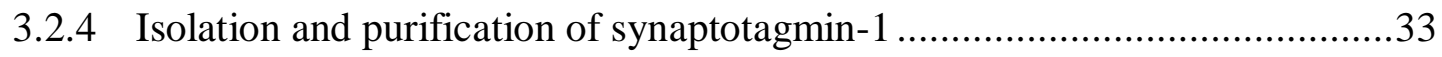

3.2.5 Reconstitution of SNARE-proteins into liposomes...................................36

3.2.6 Preparation of pore-spanning membranes (PSMs) ................................40

3.2.7 Co-reconstitution of syb 2 and syt-1 into large proteoliposomes .................41

3.2.8 Isolation and purification of chromaffin granules...................................41

3.2.9 Fluorescent labeling of chromaffin granules ........................................42 
3.2.10 Density gradient centrifugation .........................................................43

3.2.11 Phosphate determination assay ........................................................4

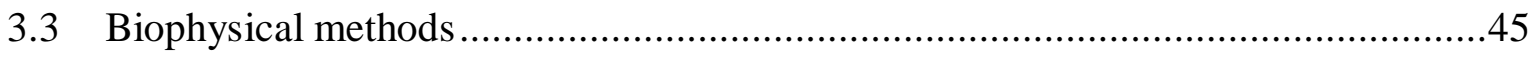

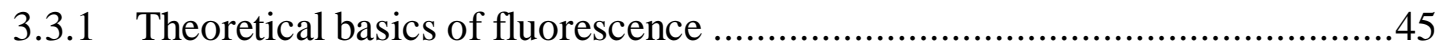

3.3.2 Förster resonance energy transfer (FRET) .........................................4

3.3.3 Fluorescence spectroscopy..............................................................49

3.3.4 Confocal laser scanning microscopy (CLSM) ............................................

3.3.5 Reflectometric interference spectroscopy (RIfS) ..................................54

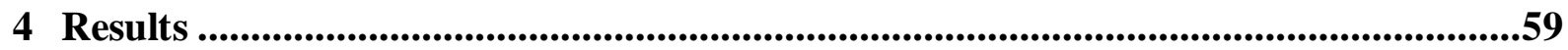

4.1 Basic properties of the artificial model system .............................................59

4.1.1 Reconstitution of SNAREs into liposomes and pore-spanning membranes...60

4.1.2 Mobility of SNAREs and lipids in pore-spanning membranes.....................61

4.1.3 Characteristics of single vesicle fusion events......................................62

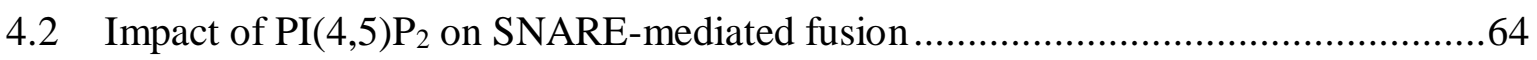

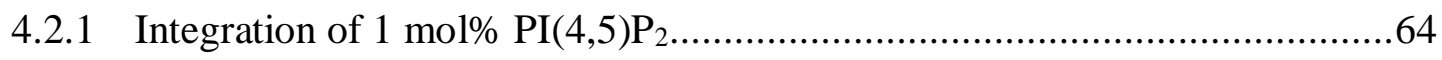

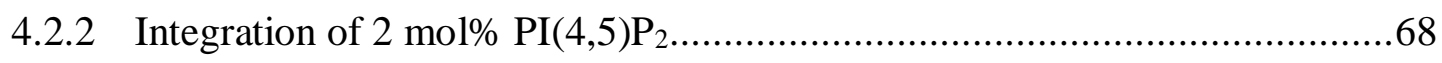

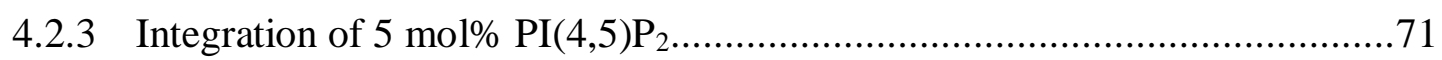

4.2.4 Comparison of SNARE-mediated single vesicle fusion on pore-spanning membranes with different $\mathrm{PI}(4,5) \mathrm{P}_{2}$ contents ........................................ 73

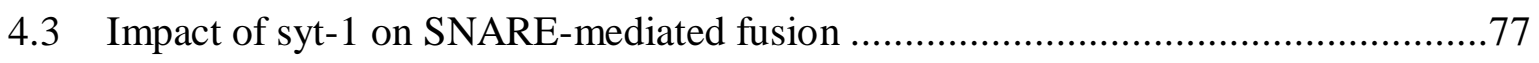

4.3.1 Isolation and purification of syt-1 …............................................... 77

4.3.2 Reconstitution of syt-1 into liposomes and analysis of the $\mathrm{Ca}^{2+}$-independent

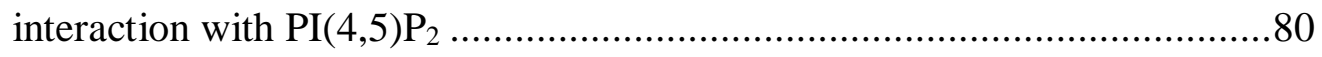

4.3.3 Co-reconstitution of syt-1 and syb 2 into liposomes and analysis of single vesicle fusion events .................................................................. 83

4.4 Verification of fusion pore formation by content labeling ...............................90

4.4.1 Analysis of the influx of Atto488-DPPE into the vesicle ...........................93

4.5 Characterization of the fusion of chromaffin granules with pore-spanning membranes 
4.5.1 Diffusion behavior of chromaffin granules on pore-spanning membranes ....98

4.5.2 Analysis of the influx of Atto488-DPPE into the CG membrane 107

5 Discussion 111

5.1 Impact of $\mathrm{PI}(4,5) \mathrm{P}_{2}$ on SNARE-mediated single vesicle fusion 111

5.2 Impact of syt-1 on SNARE-mediated single vesicle fusion 116

5.3 SNARE-mediated fusion of chromaffin granules with pore-spanning membranes . 122

5.3.1 Diffusion behavior of chromaffin granules on PSMs. 129

6 Conclusion 137

7 Appendix 139

7.1 Amino acid sequences of SNAREs and synaptotagmin-1 139

7.1.1 Amino acid sequence of synaptobrevin 2 (syb 2, aa 1-116) 139

7.1.2 Amino acid sequence of syntaxin-1A (syx-1A, aa 183-288) 139

7.1.3 Amino acid sequence of SNAP25a (aa 1-206) 139

7.1.4 Amino acid sequence of synaptotagmin-1 (syt-1, aa 1-421). 140

7.2 Calculation of the areas of catchment segments $A_{\mathrm{x}}$ for distances $r_{\mathrm{x}}>r_{\text {crit }}$

7.3 List of symbols and abbreviations 142

7.4 List of chemicals and consumables 144

7.5 List of devices and software 145

7.5.1 Devices 145

7.5.2 Software. 146

8 Bibliography 147 



\section{Introduction}

Biological membranes enclose cells and organelles, maintain their interior environment and regulate - due to selective permeability - the exchange of molecules across this border. They consist of a lipid bilayer, mainly phospho- and glycolipids as well as integral and peripheral proteins and sterols like cholesterol. Since 1972 all characterizations with regard on structure and properties of native as well as artificial membranes base on the fluid mosaic model introduced by SINGER and NICHOLSON. They described membranes in general as a twodimensional oriented solution of integral proteins in a viscous phospholipid bilayer solvent. ${ }^{[1]}$

Besides the plasma membrane, which confines the boundary of a cell, eukaryotic cells are further subdivided by intracellular membranes into many different compartments - the organelles. Those membrane-enclosed organelles constitute specific reaction compartments with characteristic differences in contents and $\mathrm{pH}$ in comparison to the cytosol. ${ }^{[2]}$ Every organelle has to be equipped with the required set of enzymes, proteins and cofactors to fulfill their specific functions. This compartmentation of the cell necessitates a highly regulated communication between the organelles to ensure selective exchange of substances. One way to exchange substances between two distinct organelles is by vesicular transport. To this end, a vesicle, filled with the cargo of interest, has to be pinched off from the donor compartment in the first step. Subsequently, this transport vesicle has to reach and recognize the target membrane of the acceptor compartment to undergo membrane fusion. Here, the content of the transport vesicle is released into the lumen of the acceptor compartment and beyond that, its membrane as well as membrane components are integrated into the target membrane. A special type of vesicular transport is the membrane fusion of a transport vesicle with the plasma membrane of a cell. In this case, the vesicle content is released into extracellular space - this process is named exocytosis.

\subsection{SNARE-mediated membrane fusion in neuronal signal transmission}

An exocytotic process of high biological relevance was found in synaptic boutons of vertebrate neurons. Here, synaptic vesicles fuse with the presynaptic membrane as a result of a transient $\mathrm{Ca}^{2+}$-influx through voltage-gated $\mathrm{Ca}^{2+}$-channels, which is evoked by incoming action 
potentials. Upon neuronal exocytosis, synaptic vesicles release their neurotransmitter content into the synaptic cleft for signal transmission to the postsynaptic neuron (Figure 1-1). ${ }^{[3]}$

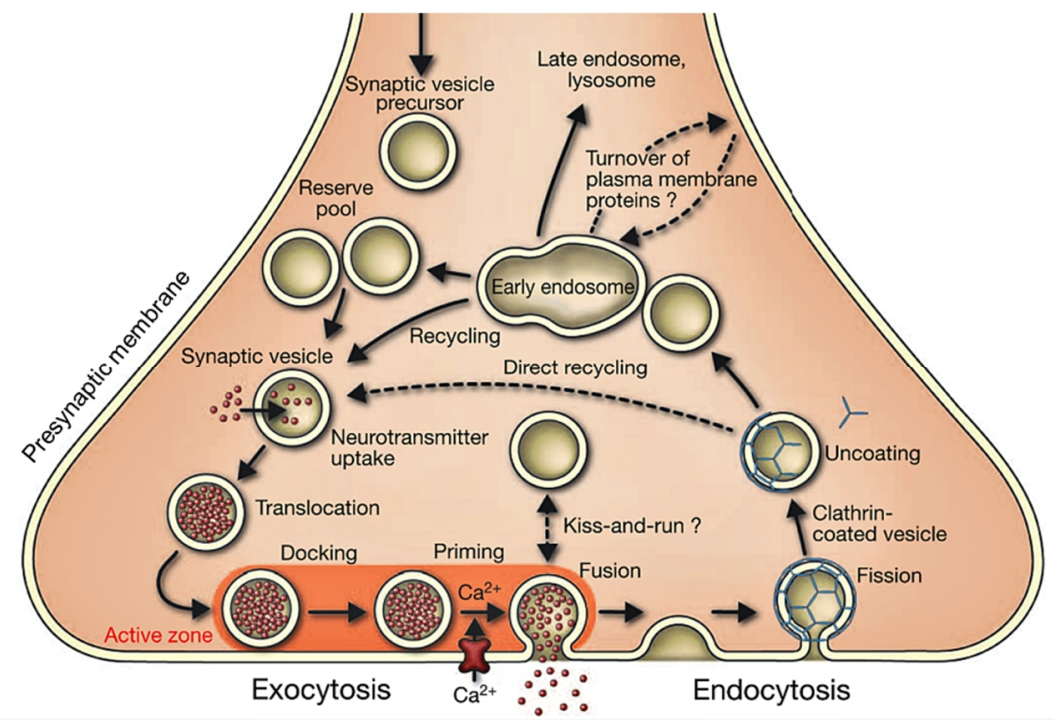

Figure 1-1 Schematic illustration of trafficking pathways in a synaptic bouton. Neurotransmitter filled synaptic vesicles (SVs) are stored in the cytoplasm, whereas a part of them is translocated to release sites in the active zone. These mature SVs, docked in the active zone, have to be activated for release in a series of metabolic energy requiring steps, called priming. The class of primed SVs is referred to as readily releasable pool, which fuses with the presynaptic membrane in a highly cooperative manner upon $\mathrm{Ca}^{2+}$ influx through voltage-gated $\mathrm{Ca}^{2+}{ }_{-}$channels evoked by incoming action potentials. SV exocytosis is followed by clathrin-mediated endocytosis and various recycling pathways.[3]

As proven by a series of knock-out experiments with genetic modified mice as well as by investigation of the mode of action of several neurotoxins, the soluble N-ethylmaleimide sensitive factor (NSF) attachment receptors (SNAREs) were identified as key players in neuronal exocytosis. The existence of SNAREs was discovered in a number of consecutive studies by ROTHMAN and coworkers in the late 1980s. ${ }^{[4],[5],[6]}$ In 1993, they were the first who isolated neuronal SNARE-proteins via affinity chromatography from bovine brain, and made the remarkable finding that the isolated SNARE-protein complex comprised of the three SNAREs syntaxin-A (syx-A), SNAP25 (synaptosome associated protein of $25 \mathrm{kDa}$ ) and synaptobrevin 2 (syb 2) in a stoichiometry of 1:1:1. ${ }^{[7]}$ Only one of these SNARE-proteins, namely syb 2, was found to be localized in the membrane of synaptic vesicles, therefore also referred to as VAMP-2 (vesicle associated membrane protein-2) or just classified as vesicular SNARE (v-SNARE). Syx-A and SNAP25, on the contrary, were found to be localized in the presynaptic membrane, which is the target membrane for the synaptic vesicle fusion. Thus, these two SNAREs were classified as target membrane SNAREs (t-SNAREs). Syb 2 and syx-A are embedded in their related membranes via $\alpha$-helical transmembrane domains (TMDs), whereas SNAP25 is anchored into the presynaptic membrane via four, posttranslationally connected palmitoyl linkers. The most characteristic feature, shared by all members of the 
SNARE-protein family is the so called SNARE-motif. This refers to a conserved homology domain of 60-70 amino acids arranged in heptad repeats which upon interaction form highly stable tetrameric four helix bundles - the SNARE-core complex (Figure 1-2). ${ }^{[8],[9],[10]}$

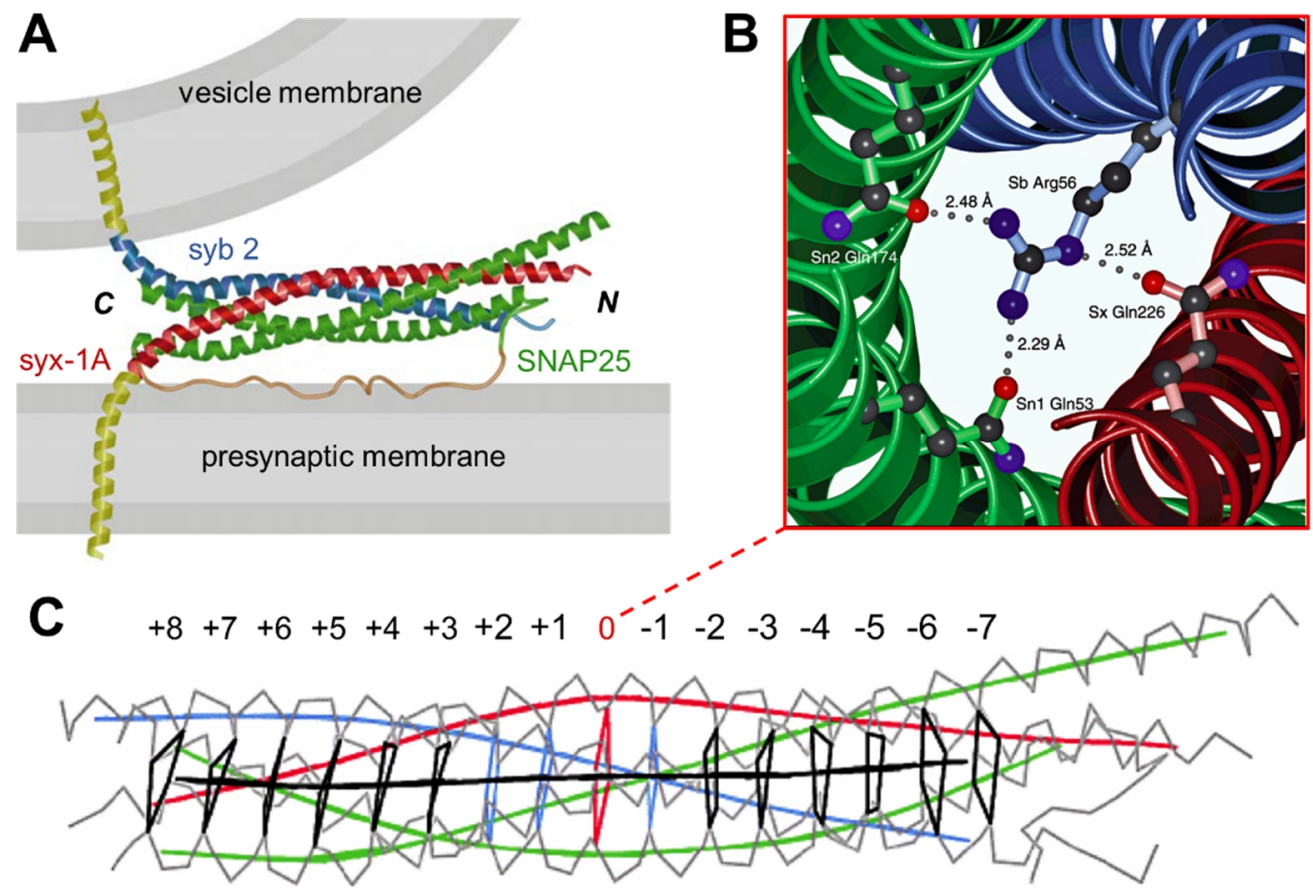

Figure 1-2 Schematic illustrations of the SNARE-core complex, based on X-ray crystallography data. (A) Hypothetic model of a trans-SNARE complex as it joins two membranes. (B) Conserved interactions in the ionic ' 0 ' layer of the SNARE-core complex. (C) Exemplary organization of interactions in 16 layers of the tetrameric four helix bundle ( $\mathrm{C}_{\alpha}$-traces in grey). Modified according to SUTTON. ${ }^{[10]}$

The two neuronal t-SNAREs, syx-1A and SNAP25, are known to be colocalized in the presynaptic membrane forming an active 1:1 complex to which syb 2, the v-SNARE, can bind. Upon contact, the three neuronal SNAREs start to assemble in trans at the $N$-terminal sites of their SNARE-motifs, followed by the formation of a tight bundle of four parallel $\alpha$-helices, each contributed by a different SNARE-motif. The SNARE-core complex assembly progresses in a 'zipper'-like fashion towards the $C$-terminal membrane anchors, thus pulling the two distinct membranes tightly together. ${ }^{[11]}$ As the formation of the tight four helix bundle is accompanied by a significant release of energy, it was determined to supply the main energy to initiate fusion. ${ }^{[12],[3]}$ After fusion, the SNARE-core complex resides in the presynaptic membrane in its low-energy cis-configuration with the TMDs of both, syx-1A and syb 2, in the same membrane. Recycling for another round of exocytosis starts with the disassembly of the cis-SNARE-core complex by the AAA+-ATPase NSF in conjunction with its SNAP (soluble NSF attachment protein) cofactor, followed by endocytosis of syb 2 .

The above described sequence of events, leading to synaptic vesicle exocytosis is a very elegant but simplistic one, only striking major observations made for neuronal signal transmission. By 
taking the high number of proteins and cofactors into account which are found to be involved in synaptic exocytosis, the picture of the exact mechanism of neurotransmitter release gets more and more enigmatic. Extensive investigations of composition and amounts of vesicle trafficking proteins in isolated synaptic boutons, performed by RIZZOLI and coworkers, resulted in a threedimensional model of an "average" synapse displaying this highly crowded compartment in atomic detail (Figure 1-3).
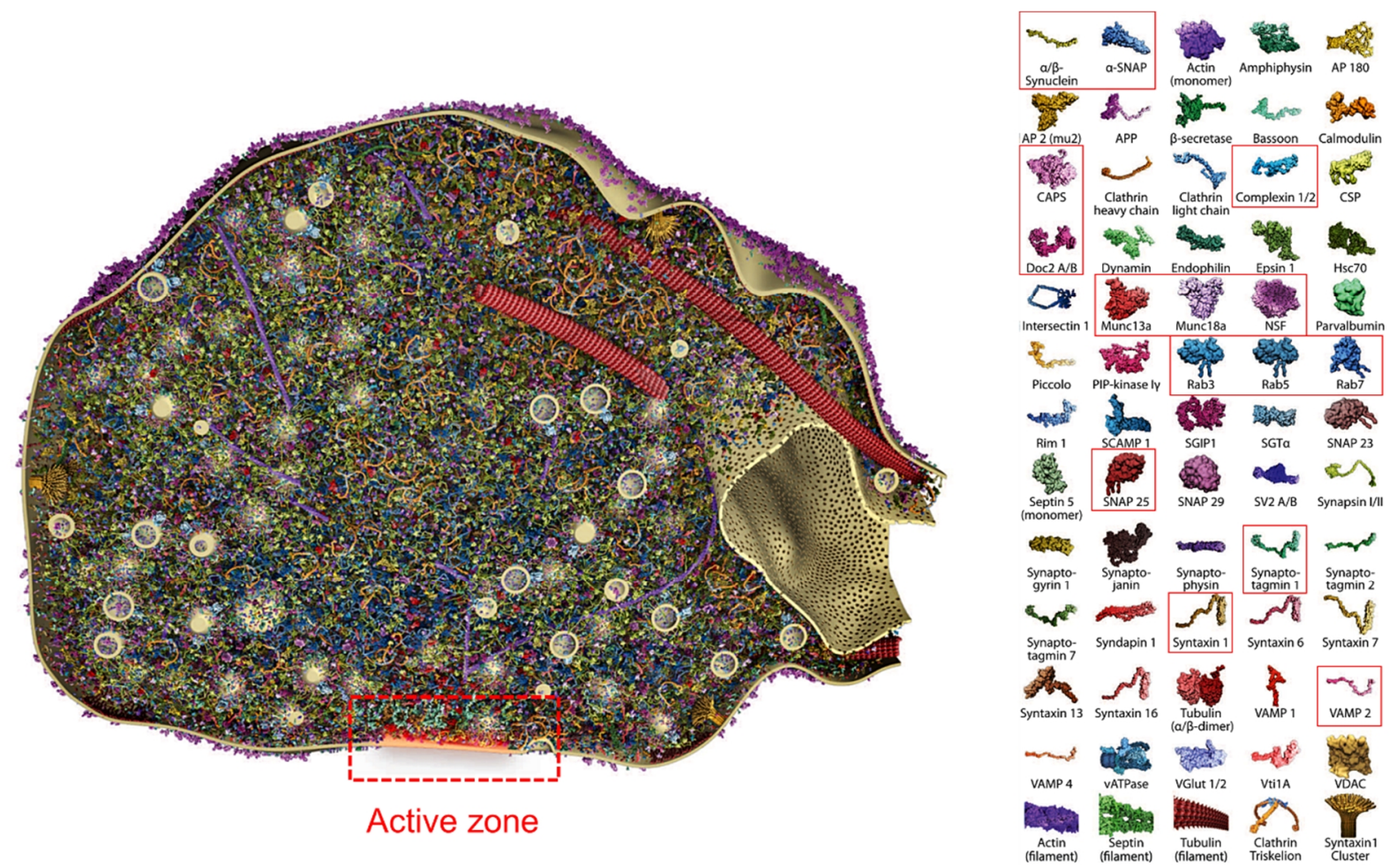

Figure 1-3 Schematic 3D model of a synaptic bouton developed by RIzzoLI and coworkers, indicating 60 different proteins in the copy numbers and localizations according to high resolution fluorescence microscopy imaging data and to the literature. The proteins highlighted in red boxes are known to be involved in neuronal exocytosis. ${ }^{[13]}$

At the first glance, it becomes obvious that in vivo experiments in such a crowded compartment make it very hard to assign proteins, which are known to be involved in neuronal exocytosis, their specific function in this process. Especially, the effect of how $\mathrm{Ca}^{2+}$-influx triggers the highly synchronic exocytosis of numerous SVs from the readily releasable pool on a submillisecond timescale is in focus of research for decades now, but the exact mechanism remains still unknown. However, the consensus has emerged that the proteins synaptotagmin-1 and complexin play an important role in $\mathrm{Ca}^{2+}$-triggered exocytosis as well as the presence of the acidic phospholipids phosphatidylserine in both membranes and phosphatidyl-4,5-bisphosphate in the presynaptic membrane. ${ }^{[14],[15],[16]}$ But to shed some light on the detailed function of individual proteins, lipids and other cofactors in neuronal signal transmission, investigation of in vitro reconstituted SNARE mediated fusion under well-defined conditions became a crucial tool. 


\subsection{In vitroreconstitution of SNARE-mediated fusion}

In the past 20 years numerous different in vitro approaches to study SNARE-mediated fusion were developed. They allow to study fusion under well-defined conditions, and therefore to focus on specific regulative proteins, lipids and cofactors in more detail in contrast to in vivo experiments. For this purpose, synaptic proteins have to be expressed in suitable hosts (e.g. $E$. coli), extracted, purified and reconstituted into artificial lipid bilayers. The most appropriate assays to analyze SNARE-mediated membrane fusion in vitro can be generally divided into two classes - ensemble and single-vesicle approaches - that are briefly introduced in the following. A more detailed and critical review about in vitro systems to investigate SNARE-mediated membrane fusion was recently published by BRUNGER et al. and is highly recommendable. ${ }^{[17]}$

\subsubsection{Ensemble vesicle fusion assays}

Ensemble fusion assays between two distinct vesicle populations can be generally divided into lipid or content mixing approaches. In 1998, WEBER et al. were the first who showed that spontaneous fusion of two distinct vesicle populations can be induced in vitro by integrating the two t-SNAREs, SNAP25 and syx-1A, into one vesicle population and the v-SNARE, syb 2, into the other population. Here, vesicle-vesicle fusion was verified by means of a lipid mixing assay, introduced by STRUCK et al. in 1981, which bases on a Förster resonance energy transfer (FRET) between the lipid-anchored fluorophores NBD-PE and Rh-PE. ${ }^{[18],[19]}$ However, membrane fusion in this case was not controllable and exhibited slow kinetics. To enhance SNARE-mediated membrane fusion in vitro POBBATI et al. developed a t-SNARE complex comprised of the two t-SNAREs and stabilized by the binding of a short fragment of the syb 2 SNARE-motif (residues 49-96), referred to as $\Delta$ N49-complex. In this complex a truncated version of syx-1A (residues 183-288) was used, lacking the regulatory $\mathrm{H}_{\mathrm{abc}}$ domain, as well as a mutant of SNAP25a, in which all cysteines were replaced by alanine to prevent cross linking or side reactions during expression. This complex provided drastically faster kinetics in vesiclevesicle bulk fusion experiments (Figure 1-4B), which was assigned to three main aspects: firstly, prevention of the spontaneous formation of an inactive syx-1A/SNAP25a 2:1-complex due to the binding of syb 2 (49-96). Secondly, preorganization of the tetrameric SNARE bundle, also evoked by the binding of syb 2 (49-96), leaving free an $\mathrm{N}$-terminal binding site for full length syb 2 and thirdly, by using the truncated version of syx-1A which excludes inactivation of syx-1A through the regulatory $\mathrm{H}_{\mathrm{abc}}$ domain. ${ }^{[20],[21],[22]}$ 
A

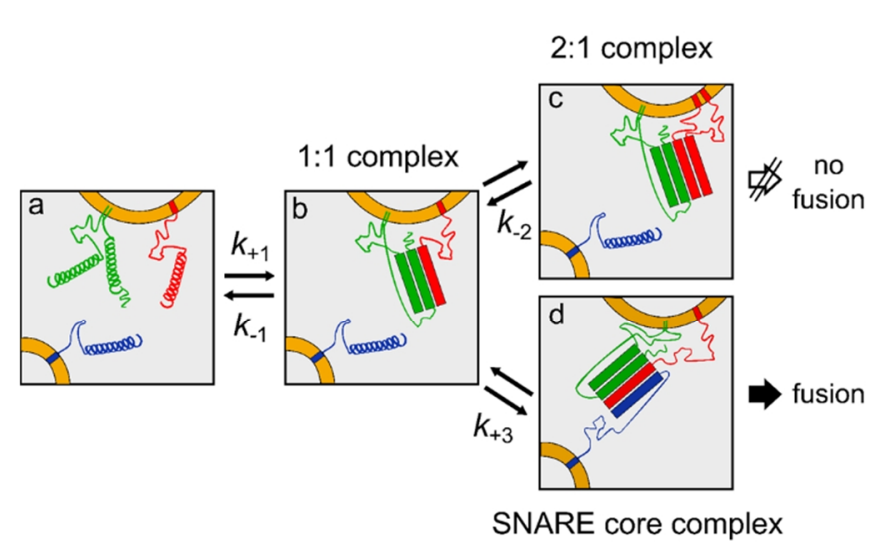

-SNAP25a "syx-1A $\square$ syb 2 asyb 2 (49-96)
B

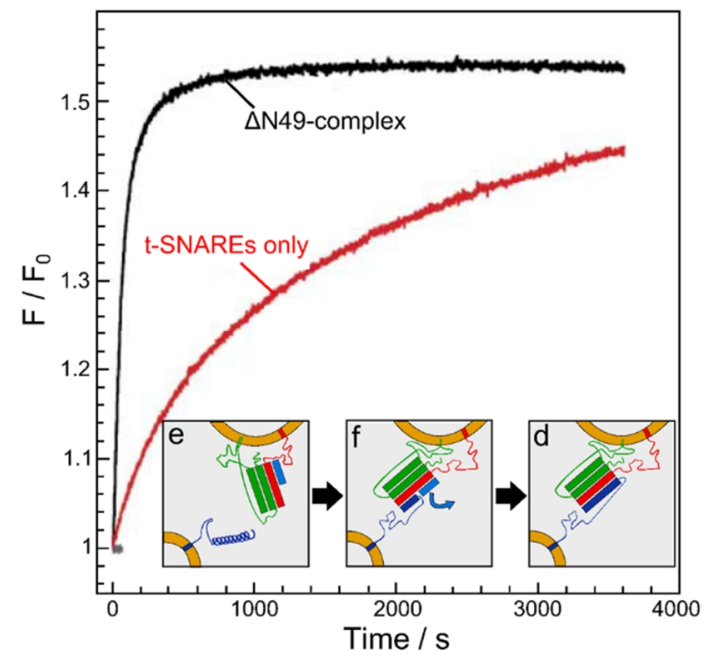

Figure 1-4 Comparison of in vitro vesicle-vesicle bulk fusion kinetics catalyzed either by the t-SNAREs only or the $\Delta$ N49-complex. (A) Schematic illustration of the kinetic model of the SNARE assembly pathway. First, the monomeric t-SNAREs syx-1A (red) and SNAP25a (green) (a) slowly form a transient 1:1 acceptor complex (b) that provides a rapid binding site for syb 2 (dark blue) or a second syx-1A. The binding of syb 2 to the 1:1 complex results in the formation of a SNARE core complex (d) to initiate fusion, whereas the binding of a second syx-1A leads to the formation of a 2:1 complex (c) which is off-pathway. Rate constants were determined for soluble portions of SNAREs with $\mathrm{k}_{+1}=6000 \mathrm{M}^{-1} \mathrm{~s}^{-1}, \mathrm{k}-1=0.01 \mathrm{~s}^{-1}, \mathrm{k}-2=0.0001 \mathrm{~s}^{-1}$ and $\mathrm{k}_{+3}=5 \cdot 10^{5} \mathrm{M}^{-1} \mathrm{~S}^{-1}$.[23] (B) Curves of liposome bulk fusion experiments analyzed by means of fluorescence spectroscopy. The black curve represents liposome fusion catalyzed by the $\Delta$ N49-complex and the red curve was recorded for liposome fusion catalyzed by t-SNAREs only. The $\triangle$ N49-complex (e) is a 1:1 acceptor complex that is stabilized by the binding of the v-SNARE fragment syb 2 (49-96) (light blue) which can be displaced by syb 2 (f) resulting in a fusion initiating SNARE core complex. Modified according to PoBBATI.[21]

The development of the preorganized $\Delta \mathrm{N} 49$-complex was a major step forward regarding the kinetics of SNARE-mediated liposome bulk fusion in vitro but the significant drawback of ensemble vesicle fusion assays that one cannot distinguish between different fusion states remained. Neither with inner leaflet lipid mixing assays, where the fluorophores in the outer leaflet are quenched, ${ }^{[24],[25]}$ nor with content mixing assays ${ }^{[22],[26],[27]}$ one can determine the percentages of simultaneous occurring fusion states like docking, hemifusion and full fusion. But to investigate SNARE-mediated membrane fusion in detail, the resolution to monitor the intermediate states is paramount.

\subsubsection{Single vesicle fusion assays}

To overcome the disadvantage of ensemble vesicle fusion assays that can only be used to monitor the average fusion behavior of liposomes (often in the order of AVOGADRO's number), single vesicle fusion assays have been developed. The ability to investigate fusion on the single particle level allows one to perform statistical analysis of a population of individual fusion trajectories to gain insights into the detailed mechanism of SNARE-mediated membrane fusion. 
Approaches to analyze fusion on the single vesicle level can be classed as vesicle-vesicle or vesicle-planar bilayer assays.

There are a multitude of approaches to investigate vesicle-vesicle fusion on the single particle level. In order to analyze vesicle-vesicle fusion of "free" vesicles, a method was developed to observe single vesicle species diffusing in and out of a small confocal volume (femtoliter) by alternating-laser excitation (ALEX) with donor- and acceptor-dye excitation lasers. ${ }^{[28],[29]}$ In this method, v- and t-SNARE vesicles were labeled with donor and acceptor lipid dyes, giving rise to characteristic fluorescent bursts by which a discrimination between a single vesicle, a pair of docked vesicles or a hemifused/fused vesicle was possible. Another approach to analyze the fusion behavior of freely diffusing vesicles on the single particle level is based on fluorescence cross-correlation spectroscopy (FCCS) in combination with a donor fluorescence lifetime analysis. ${ }^{[30]}$ Similar to the ALEX experiments, donor- and acceptor-dye labeled vesicles are mixed and imaged by confocal microscopy in a small confocal volume but, in this case, simultaneous excitation of both fluorophores was applied. By separate detection of the two distinct fluorescence signals a fluorescence cross-correlation function as well as a fluorescence lifetime can be calculated. Both, the ALEX and the FCCS method suffer from the fact that only a few vesicles pass through the excitation volume at any given time, limiting the time-resolution of these methods to $\sim 30 \mathrm{~s}$ and $\sim 90 \mathrm{~s}$, respectively. ${ }^{[17]}$ Another drawback of these two approaches is the fact that one cannot distinguish between the full and hemifused state. In order to achieve that and to improve the time-resolution, single particle assays were developed based on simultaneous detection of content and lipid mixing (cf. Figure 1-5).
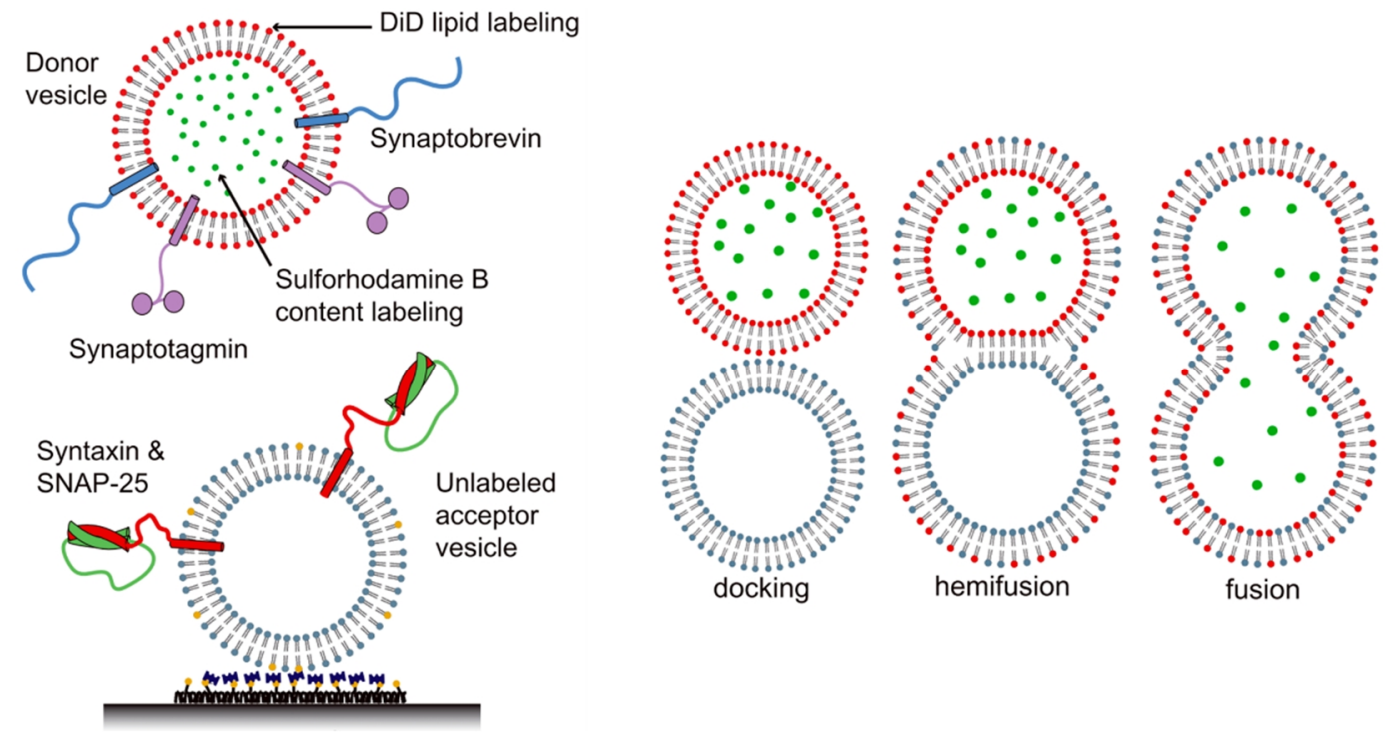

Figure 1-5 Schematic illustration of the setup to simultaneously monitor content and lipid mixing of vesiclevesicle fusion on the single particle level. This approach enables to distinguish between the fusion states docking, hemifusion and full fusion. ${ }^{[17]}$ 
For this purpose, t-SNARE vesicles were tethered to a passivated surface by neutravidin-biotin linkages, content and membrane labeled v-SNARE vesicles were added and single vesiclevesicle fusion events were detected by total internal reflection fluorescence microscopy (TIRFM). This assay enables to analyze fusion on a sub-100 ms time scale and allows to discriminate between a variety of different vesicle-vesicle fusion events by simultaneously monitoring lipid and content mixing as schematically depicted in Figure 1-5. ${ }^{[31],[32]}$

Although single vesicle-vesicle assays allow to discriminate between fusion intermediates and offer a high time-resolution, they do not resemble the situation found in a synaptic bouton quite well, where highly curved synaptic vesicles fuse with a rather planar presynaptic membrane. For a better imitation of the membrane geometries found in a synapse, a second class of single particle assays were developed by several groups in which fusion between v-SNARE doped vesicles and a t-SNAREs containing planar lipid bilayer was in the focus of investigation. Here, the definition of a planar lipid bilayer is relatively broad including giant unilamellar vesicles $\left(\right.$ GUVs) ${ }^{[33],[34],[35]}$ as well as different kinds of solid supported membranes (SSMs). In case of SSMs, most approaches were based on TIRFM to detect single vesicle fusion either by lipid or content mixing or both. ${ }^{[36]-[40]}$ Besides rather inconsistent findings among these assays concerning the docking lifetimes and SNAP25 dependencies, all suffer from the poor lateral mobility of the membrane components, in particular of the proteins reconstituted into the SSM. Moreover, the SSMs lack a second aqueous compartment due to the close contact to the solid support and therefore do not provide enough space for the incoming lipid material upon fusion. To overcome the reduced lateral mobility of membrane components in SSMs on glass, approaches were developed to prepare planar membranes decoupled from the support by polyethylene glycol brushes. ${ }^{[41],[42]}$ But even in these planar membranes on polymer cushions a relatively low diffusion coefficient of $D=0.14 \mu \mathrm{m}^{2} / \mathrm{s}$ was determined for the embedded t-SNARE complex. Beyond that, all these SSMs, including membranes on polymer cushions, do not provide aqueous compartments on both sides of the lipid bilayer as present in the plasma membrane of a synapse.

As there are still a number of drawbacks associated with the current state-of-the-art planar membrane architectures used to analyze SNARE-mediated fusion in vitro, the demand for an enhanced artificial system remains. Planar pore-spanning membranes (PSMs) - an artificial lipid bilayer system decisively developed and improved in the group of CLAUDIA STEINEM were recently shown to be a suitable system to investigate SNARE-mediated fusion on a single particle level. ${ }^{[43],[44],[45]}$ PSMs are composed of a lipid bilayer which is spanned over a hydrophilically functionalized porous substrate with regular arranged cylindrical holes of a 
defined diameter. This results in two distinct parts of the planar lipid bilayer, the free-standing part (f-PSM) which is spanned over the pores to seal them and the solid supported part (s-PSM) which covers the area in between the pores (cf. Figure 1-6B). Together, these two distinct membrane parts form a continuous lipid bilayer as schematically depicted in Figure 1-6C which provides high protein mobility in the f-PSM as well as the space for incoming lipid material during fusion. ${ }^{[44],[45]}$
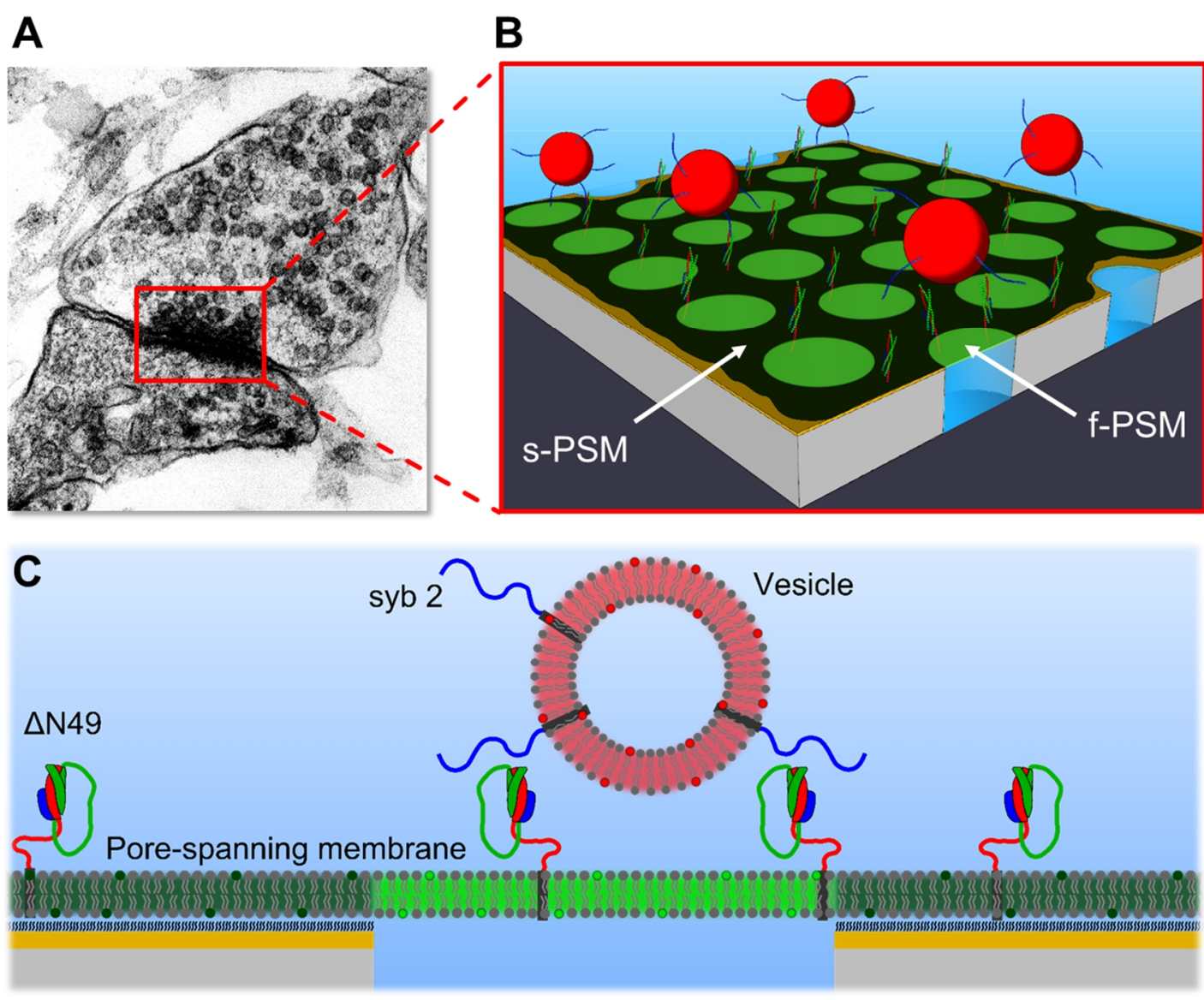

Figure 1-6 (A) Electron micrograph of a chemical synapse - the biological idol.[46] (B)+(C) Schematic illustrations of the model system of pore-spanning membranes (PSMs) to investigate SNARE-mediated single vesicle fusion in 3D and in cross section. The PSM (green) is fluorescently labeled with OG-DPPE and doped with the t-SNARE acceptor complex $\triangle$ N49, whereas the proteoliposomes (red) contain TxR-DPPE as a fluorescent marker and the v-SNARE syb 2.

As PSMs offer clear advantages compared to other artificial planar lipid bilayer system, they were already used to analyze SNARE-mediated membrane fusion on the single vesicle level. The assay, in this case, was based on lipid mixing and enabled to discriminate between the key steps of fusion (docking, intermediate fusion, full fusion). As an in vitro fusion machinery, the t-SNARE acceptor complex $\triangle \mathrm{N} 49$ was reconstituted into PSMs that were labeled with the fluorescent marker OregonGreen-DPPE (OG-DPPE) and the v-SNARE syb 2 was integrated into large unilamellar vesicles (LUVs) doped with TexasRed-DPPE (TxR-DPPE). Single vesicle fusion events were monitored with an upright confocal laser scanning microscope, 
where the two fluorescent dyes were excited with the same laser line $(488 \mathrm{~nm})$ but detected in two separate channels. The results of this study clearly demonstrated the unique potential of this model system. However, it offers many starting points for improvement either from the technical side or the biological. From the technical side, it would be preferable to monitor both content and lipid mixing simultaneously with a high time-resolution. From the biological side, on the contrary, it would be very interesting to integrate more lipids and proteins that are known from in vivo experiments to play a central role in SNARE-mediated fusion to analyze their impact on fusion in vitro. From the literature and experiments that are summarized in the model of an average synaptic bouton (cf. Figure 1-3) by RIZZOLI and coworkers, it becomes obvious that signal transmission is a very complex biological process that needs to be highly regulated by a multitude of lipids, proteins and other co-factors. But in connection with $\mathrm{Ca}^{2+}$-triggered synchronic neurotransmitter release there are two outstanding key players - namely synaptotagmin-1 and $\mathrm{PI}(4,5) \mathrm{P}_{2}$. The analysis of the impact of these two components on SNARE-mediated fusion in vitro was in the focus of this work, therefore they will be introduced in more detail in the following chapters.

\subsection{The role of $\mathrm{PI}(4,5) \mathrm{P}_{2}$ in neuronal exocytosis}

Phosphatidylinositol-4,5-bisphosphate $\left(\mathrm{PI}(4,5) \mathrm{P}_{2}\right.$, structure cf. Table 3-1) is with $\sim 1 \%$ of total lipids the most abundant phosphoinositide in the inner leaflet of mammalian plasma membranes. ${ }^{[47]}$ Early studies stated the role of $\mathrm{PI}(4,5) \mathrm{P}_{2}$ as a second messenger in the phospholipase-C signaling pathway. ${ }^{[48]}$ However, until today, the list of cellular functions of $\mathrm{PI}(4,5) \mathrm{P}_{2}$ is growing rapidly. Most of the functions rely on electrostatic $\mathrm{PI}(4,5) \mathrm{P}_{2}$-protein interactions and among these are membrane trafficking, attachment of the cytoskeleton to the plasma membrane, activation of enzymes as well as endo- and exocytosis. ${ }^{[48],[49]}$ Especially the role of $\mathrm{PI}(4,5) \mathrm{P}_{2}$ in neuronal exocytosis is in focus of current research. Here, a common vision emerged in which $\mathrm{PI}(4,5) \mathrm{P}_{2}$ mediates clustering of the t-SNARE syntaxin-1A (syx-1A) into microdomains serving as hot spots for synaptic vesicle priming and fusion. ${ }^{[49],[50],[51],[52],[53]}$ The results demonstrate that $\mathrm{PI}(4,5) \mathrm{P}_{2}$ is the predominant lipid in syx-1A microdomains, found in the inner-leaflet of neuroendocrine PC12 cells (Figure 1-7). 

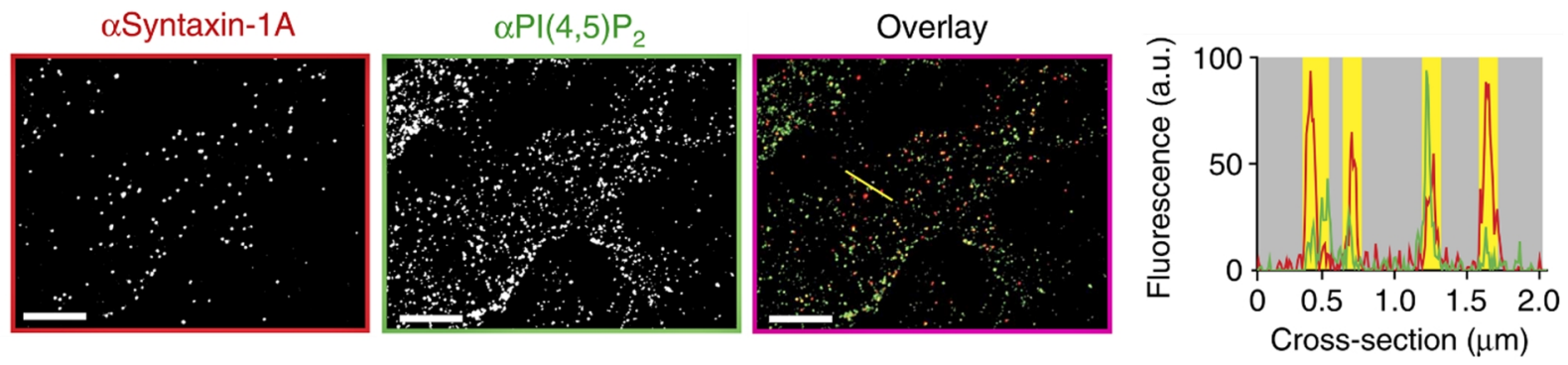

Figure 1-7 Syntaxin-1A (red frame) colocalizes with $\mathrm{PI}(4,5) \mathrm{P}_{2}$ (green frame) in plasma membrane sheets of PC12 cells. Syntaxin-1A as well as $\mathrm{PI}(4,5) \mathrm{P}_{2}$ were immunostained and imaged by two-color STED microscopy (scale bars: $2 \mu \mathrm{m}$ ). The graph shows the fluorescence intensity profiles of the cross-section depicted as yellow bar in the overlay (syntaxin-1A: red line, $\mathrm{PI}(4,5) \mathrm{P}_{2}$ : green line). ${ }^{[52]}$

In these microdomains of about $70 \mathrm{~nm}$ in diameter, $\mathrm{PI}(4,5) \mathrm{P}_{2}$ was found to accumulate over $80 \%$ of the total surface area. ${ }^{[51]}$ It is believed, that the microdomain formation is based on electrostatic interactions between the anionic headgroup of $\mathrm{PI}(4,5) \mathrm{P}_{2}$ and the polybasic juxtamembrane sequence (260-KARRKK-265) of syx-1A (Figure 1-8). ${ }^{[52]}$ Moreover, it was found that the syx-1A microdomain formation in PC12 cells as well as in vitro is $\mathrm{Ca}^{2+}$ independent but can be enhanced by increasing the $\mathrm{Ca}^{2+}$ concentration. ${ }^{[16]}$ This leads to the formation of new microdomains and to the connection of multiple $\mathrm{PI}(4,5) \mathrm{P}_{2} / \mathrm{syx}-1 \mathrm{~A}$ microdomains to larger mesoscale domains which was analyzed by atomic force microscopy (AFM) and two-color stimulation emission depletion (STED) nanoscopy. ${ }^{[54]}$

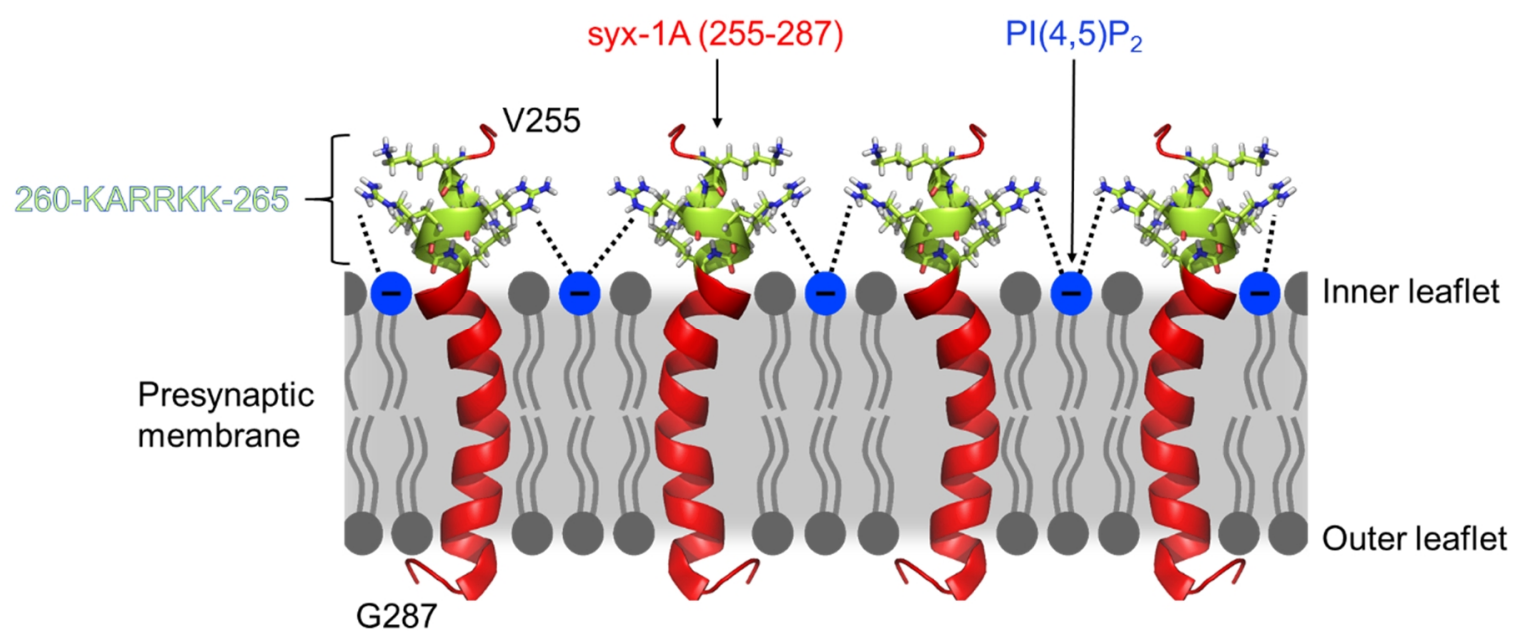

Figure 1-8 Schematic illustration of $\mathrm{PI}(4,5) \mathrm{P}_{2}$ (blue) induced sequestering of syx-1A (red; PDB ID 2M8R) in the presynaptic membrane. The $\mathrm{Ca}^{2+}$-independent cluster formation is based on electrostatic interactions between the polybasic juxtamembrane sequence 260-KARRKK-265 of syx-1A and the anionic headgroup of $\mathrm{PI}(4,5) \mathrm{P}_{2}$ and can be enhanced by $\mathrm{Ca}^{2+-i o n s . ~}$ 


\subsection{The role of synaptotagmin-1 in $\mathrm{Ca}^{2+-e v o k e d ~ e x o c y t o s i s ~}$}

In a chemical synapse, action potentials give rise to a $\mathrm{Ca}^{2+}$-influx into the synaptic bouton through voltage-gated $\mathrm{Ca}^{2+}$-channels. This increase in intracellular $\mathrm{Ca}^{2+}$-concentration is accompanied by the fusion of synaptic vesicles with the presynaptic membrane to release neurotransmitter molecules into the synaptic cleft. After passing the synaptic cleft, the neurotransmitter molecules bind to receptors in the postsynaptic neuron to induce a new action potential. ${ }^{[55],[56],[57]}$

Besides $\mathrm{Ca}^{2+}$-dependent exocytosis, $\mathrm{Ca}^{2+}$-independent exocytosis was also observed in a few special types of synapses. ${ }^{[58]}$ However, the $\mathrm{Ca}^{2+}$-independent process was found to be spontaneous and asynchronous, whereas the $\mathrm{Ca}^{2+}$-triggered exocytosis leads to a highly synchronous secretion of neurotransmitter molecules and was determined to be the most abundant mechanism in neurotransmission. ${ }^{[59],[60]}$ Therefore, the existence of one or more molecular sensors for $\mathrm{Ca}^{2+}$ is a basic prerequisite for signal transmission between neurons. In vertebrate synapses, synaptotagmin-1, -2 and -9 were found to be the main candidates for a $\mathrm{Ca}^{2+}$ sensor to trigger fast synchronous neurotransmitter release. ${ }^{[61],[62],[63]}$

Up till now, 17 isoforms of the synaptotagmin (syt) protein family were discovered, ${ }^{[64]}$ but as the focus of this work was on syt-1 and its impact on in vitro SNARE-mediated membrane fusion, only this isoform will be characterized in detail. Syt-1 is a $\mathrm{Ca}^{2+}$-binding protein, which is specifically localized in the membrane of synaptic vesicles and that of secretory granules in endocrine cells. ${ }^{[65]}$ It is comprised of a short unstructured $N$-terminal intravesicular part, followed by an $\alpha$-helical transmembrane domain (TMD) and its main part, the cytosolic region. The cytosolic part of syt- 1 harbors the two $\mathrm{Ca}^{2+}$-binding domains, $\mathrm{C} 2 \mathrm{~A}$ and $\mathrm{C} 2 \mathrm{~B}$, which can bind 3 and $2 \mathrm{Ca}^{2+}$ ions, respectively. The aspartate residues that coordinate the $\mathrm{Ca}^{2+}$-ions are localized in flexible loops at the distal tips of the C2-domains (Figure 1-9). From isothermal titration calorimetry (ITC) experiments with isolated $\mathrm{C} 2 \mathrm{~A}$ and $\mathrm{C} 2 \mathrm{~B}$, models as well as affinities for $\mathrm{Ca}^{2+}$-binding were obtained. The study was performed by RADHAKRISHNAN et al. and resulted for the $\mathrm{C} 2 \mathrm{~A}$ domain in a three-site sequential binding model with dissociation constants of $K_{\mathrm{D} 1}=119 \mu \mathrm{M}, K_{\mathrm{D} 2}=465 \mu \mathrm{M}$ and $K_{\mathrm{D} 3} \approx 1.7 \mathrm{mM}$. For the C2B domain, in contrast, a one-site binding model was obtained, indicating two independent binding sites for $\mathrm{Ca}^{2+}$ with dissociation constants of $K_{\mathrm{D} 1} \approx K_{\mathrm{D} 2} \approx 200 \mu \mathrm{M} .{ }^{[66]}$ Figure 1-9 shows ribbon plots of both $\mathrm{C} 2$ domains in their $\mathrm{Ca}^{2+}$-bound state obtained by nuclear magnetic resonance spectroscopy (NMR) and X-ray crystallography. ${ }^{[67],[68]}$ 

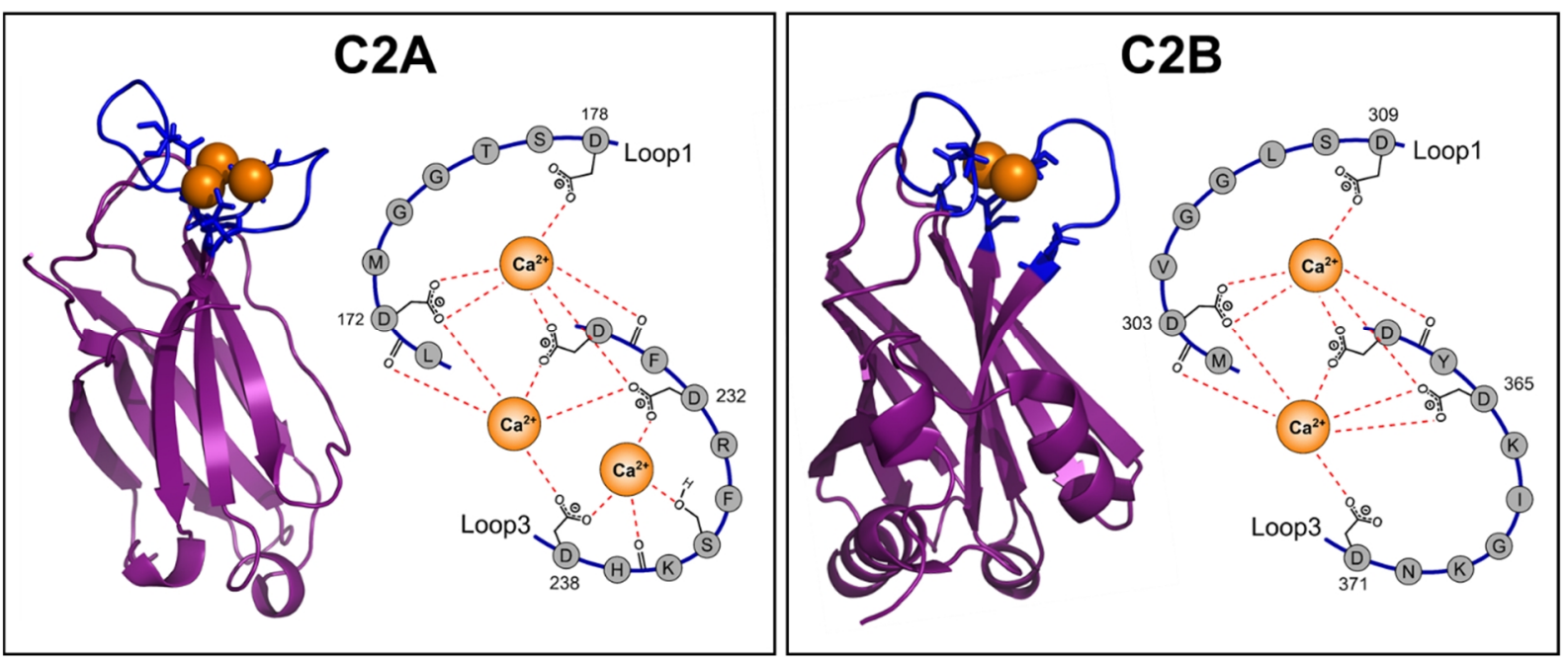

Figure 1-9 Ribbon plots and schematic illustrations of the $\mathrm{Ca}^{2+}$-binding loops of the C2A (PDB ID 1BYN) and C2B (PDB ID 1UOW) domains of synaptotagmin-1. The C2A and C2B domains exhibit three and two binding sites for $\mathrm{Ca}^{2+}$, respectively. The residues that coordinate the $\mathrm{Ca}^{2+}$-ions (orange) are localized in flexible loops at the distal tips of the C2-domains and are depicted in blue. Modified according to CHAPMAN.[69]

As the coordination sites of the bound $\mathrm{Ca}^{2+}$-ions are occupied incompletely by the $\mathrm{C} 2$-domains, the $\mathrm{Ca}^{2+}$-affinities for the isolated domains as well as for the $\mathrm{C} 2 \mathrm{AB}$ tandem domain $\left(K_{\mathrm{D}} \neq 50 \mu \mathrm{M}-3 \mathrm{mM}\right)^{[66]}$ were determined to be relatively low. Here, the interaction with lipids in the target membrane comes into play. In 1990, PERIN et al. were the first who showed that the C2-domains of syt-1 specifically interact with acidic phospholipids and beyond that they suggested that $\mathrm{Ca}^{2+}$-binding may also play a role in this interaction. This hypothesis was confirmed by BROSE et al., who observed a drastic increase in $\mathrm{Ca}^{2+}$-binding in the presence of liposomes containing up to $50 \mathrm{~mol} \%$ of phospholipids with the acidic headgroup phosphatidylserine (PS). ${ }^{[70]}$ Further investigations of the syt-1/membrane-interactions reinforced the model of a $\mathrm{Ca}^{2+}$-dependent insertion of the $\mathrm{C} 2$-domains into the target membrane as a result of negative surface charge neutralization. ${ }^{[71]}$ It was concluded that $\mathrm{Ca}^{2+}$-binding changes the surface charge of the binding loops from negative to positive. This electrostatic switch enables the C2-domains to interact with negatively charged phospholipids in the target membrane, which complete the coordination spheres of the bound $\mathrm{Ca}^{2+}$-ions leading to enhanced $\mathrm{Ca}^{2+}$-affinities (Figure 1-10). 
$\mathrm{C} 2 \mathrm{~A}$

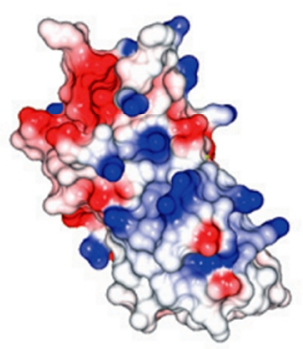

$\mathrm{C} 2 \mathrm{~B}$

00

$+\mathrm{Ca}^{2+}$
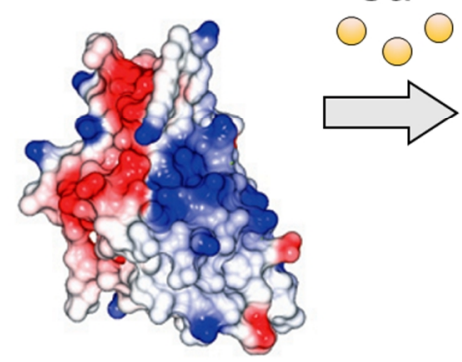

$\mathrm{C} 2 \mathrm{~A}$

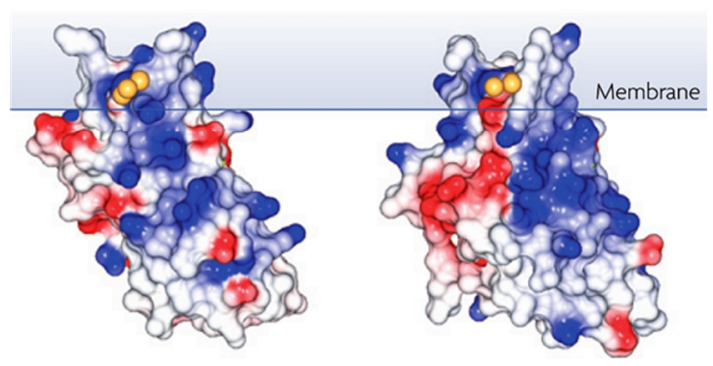

Figure 1-10 Schematic illustration of the $\mathrm{Ca}^{2+-}$ dependent membrane interactions of the C2-domains. $\mathrm{Ca}^{2+-}$ ions (yellow spheres) are coordinated by aspartate residues, which are localized in the flexible binding loops on top of synaptotagmin's C2A and C2B domains. $\mathrm{Ca}^{2+-}$ binding switches the surface potential of the binding sites from negative to positive and enables the C2-domains to interact with acidic phospholipids in membranes and beyond that to partly insert into the membrane. Modified according to MARTENS. ${ }^{\text {[72] }}$

Recent findings of HONIGMANN et al. refined this model of syt-1 interactions drastically. ${ }^{[16]}$ They determined the crystal structure of the C2B domain bound to PS and developed a highresolution model of how syt-1 can bridge two different membranes. In this model, it is suggested that $\mathrm{PI}(4,5) \mathrm{P}_{2}$, which was found to be the dominant lipid in microdomains of syx-1A (cf. Chapter 1.3), acts as a connector between the $\mathrm{C} 2 \mathrm{~B}$ domain and syx-1A in a $\mathrm{Ca}^{2+}$ independent manner. This interaction was found to be selective and of electrostatic nature between the polyanionic headgroup of $\mathrm{PI}(4,5) \mathrm{P}_{2}$ and the polybasic sequences in syx-1A (260KARRKK-265) and C2B (324-KKKK-327). This $\mathrm{Ca}^{2+}$-independent interaction of the $\mathrm{C} 2 \mathrm{~B}$ domain was proposed to enhance the recruitment of vesicles to $\mathrm{PI}(4,5) \mathrm{P}_{2} /$ syx-1A-microdomains in PC12 cells serving as hot spots for exocytosis. By increasing the $\mathrm{Ca}^{2+}$-concentration, the tandem $\mathrm{C} 2 \mathrm{AB}$ domain was found to tether the two opposite membranes. As a prerequisite for $\mathrm{Ca}^{2+}$-dependent tethering, the liposome membrane had to contain at least $5 \%$ of the anionic lipid PS in accordance with previous findings of high affinities of the C2-domains to acidic phospholipids in their $\mathrm{Ca}^{2+}$-bound state. A schematic illustration of the proposed syt-1 binding model by HONIGMANN et al., including a $\mathrm{Ca}^{2+}$-independent vesicle recruitment to sites of exocytosis, followed by $\mathrm{Ca}^{2+}$-dependent tethering of the two opposite membranes is shown in Figure 1-11. 


\section{$\mathrm{Ca}^{2+}-$ independent binding}

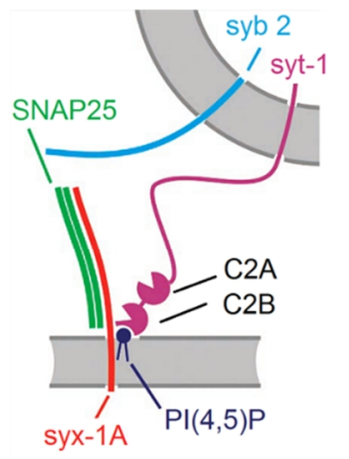

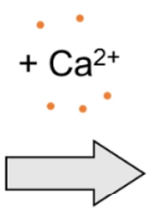

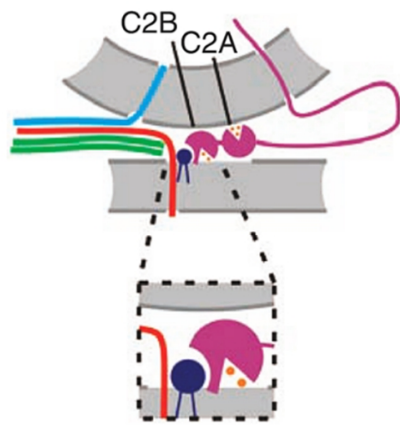

parallel orientation of the $\mathrm{C} 2 \mathrm{~B}$ domain
$\mathrm{Ca}^{2+}$-dependent tethering

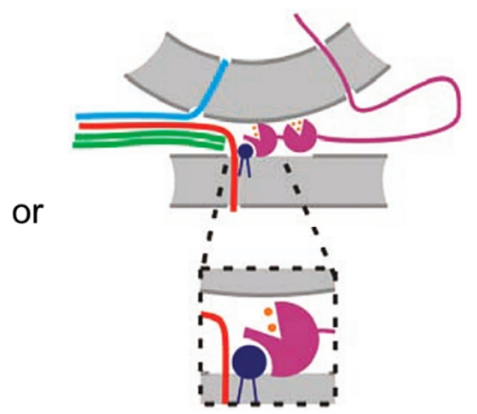

perpendicular orientation of the $\mathrm{C} 2 \mathrm{~B}$ domain

Figure 1-11 Schematic illustration of the proposed model by HoNIGMANN et al. of vesicle recruitment and tethering induced by the $\mathrm{C} 2$-domains of syt-1. (left) $\mathrm{Ca}^{2+}$-independent binding of the polybasic sequence of synaptotagmins $\mathrm{C} 2 \mathrm{~B}$ domain to microdomains enriched in $\mathrm{PI}(4,5) \mathrm{P}_{2}$ and syx-1A. (right) $\mathrm{Ca}^{2}{ }^{2-}$ dependent tethering by synaptotagmins $\mathrm{C} 2 \mathrm{~A}$ and $\mathrm{C} 2 \mathrm{~B}$ domains, in which the $\mathrm{C} 2 \mathrm{~B}$ domain is oriented parallel, meaning that the $\mathrm{Ca}^{2+}$-binding site interacts with the plasma membrane, or oriented perpendicular, signifying a back binding to the vesicle membrane. Modified according to HONIGMANN. ${ }^{[16]}$

\subsection{Chromaffin granules and SNARE-mediated exocytosis}

The fight-or-flight response is a physiological reaction that occurs under conditions of fear or stress and leads to a rapid surge of adrenal catecholamines in the bloodstream. It was first described by the physiologist WALTER B. CANNON in 1915 as a visceral function of the sympathetic nervous system to prepare animals for fighting or fleeing in potentially dangerous situations. ${ }^{[73],[74]}$ This response is thought to be initiated by a set of central autonomic neurons in the hypothalamus and brainstem providing dual input to the sympathetic preganglionic neurons to regulate adrenal medullary secretion and cardiovascular function. ${ }^{[75]}$ Taken together, both, the cardiac and adrenal sympathetic outflow systems, prepare the body for peak physical condition, for example by increasing the blood flow to muscles, the heart rate and the blood sugar level. ${ }^{[76]}$

As mentioned before, the adrenal medulla (center of the adrenal gland) is the major sympathetic effector in this process. It consists of cells that secrete hormones and neuroactive peptides into systemic circulation upon extreme emotional or physical situations via exocytosis. Those neuroendocrine cells are called chromaffin cells and within these cells the neuroactive compounds are stored in dense-core secretory granules - referred to as chromaffin granules (CGs). ${ }^{[77],[78]}$ CGs represent a very interesting model to study SNARE-mediated membrane fusion because they use the same set of SNARE-proteins for recognition and fusion as neurons. ${ }^{[79],[80],[81]}$ Through stimulation of the adrenal medulla CGs fuse in a $\mathrm{Ca}^{2+}$-triggered 
process with the plasma membrane of the chromaffin cell to release their neuroactive content into circulation. It was found that CGs exhibit a great variety of exo- and endocytic modes depending on the stimulus intensity which is directly coupled to the physiological demand. ${ }^{[82],[83],[76],[84]}$ Exocytic modes that are discussed in this context are the "kiss-and-run" mode which only allows the release of catecholamines and other small molecules through a narrow fusion pore and the "cavicapture" (granule cavity capture) mode where the initial fusion pore expands to release even small peptides but re-seals after a certain time. During these two modes the granule $\Omega$-shape remains almost intact and allows a fast endocytic recycling, whereas during "full collapse" exocytosis, granules lose their $\Omega$-shape completely and merge with the plasma membrane to release their complete content into the extracellular space. ${ }^{[85],[86],[84]}$

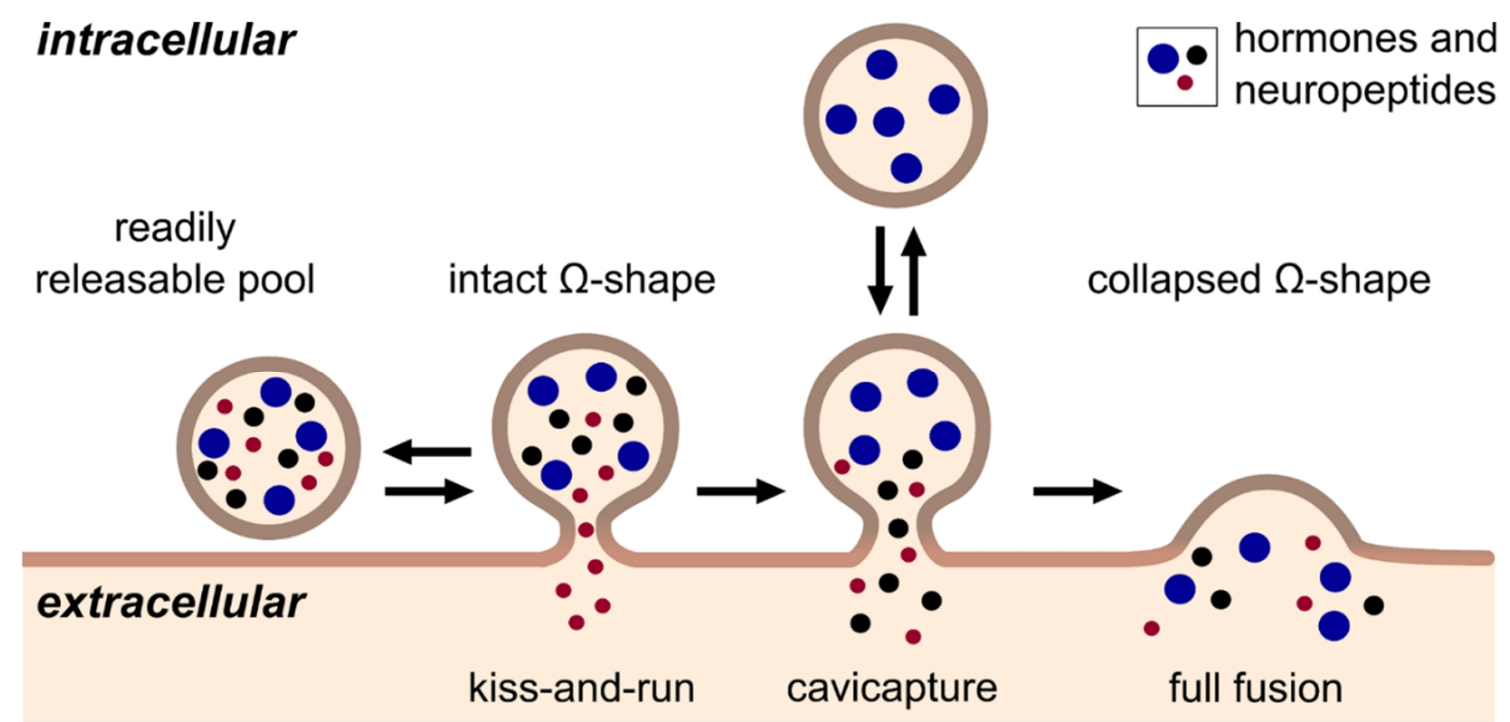

Figure 1-12 Different modes of exocytosis in neuroendocrine cells. Mature granules, which are primed at the plasma membrane, constitute the readily releasable pool. Large proteins (blue dots), small neuropeptides (black dots) and small hormones like catecholamines (red dots) can be released differentially depending on the stimulus intensity. During "kiss-and-run" mode, only small molecules (catecholamines) are released through a narrow fusion pore, whereas "cavicapture" (granule cavity capture) allows the partial release of small neuropeptides. As the granule $\Omega$-shape remains almost intact in these two modes, retrieval of intact granules is easily conceived. In case of full fusion however, the granule $\Omega$-shape completely collapses into the plasma membrane to release the whole intra-granular content into the extracellular space. This mode is caused by strong stimuli and has to be compensated by elaborate endocytosis. Modified according to HouY.[86]

This great variety in exocytic fusion behavior, depicted in Figure 1-12, predestinates CGs to be analyzed in detail in a suited model system on the single vesicle level. 


\section{Scope}

At the heart of neuronal exocytosis is the $\mathrm{Ca}^{2+}$-triggered fusion of synaptic vesicles from the readily releasable pool with the presynaptic membrane upon incoming action potentials. This highly regulated synchronous secretion of neurotransmitter filled vesicles leads to stimulus conduction and was determined to take place on a sub-millisecond timescale. ${ }^{[87],[88]}$ Proteins that were clearly identified to play a crucial role in this process of membrane fusion are the SNAREs (soluble N-ethylmaleimide-sensitive-factor attachment receptors), which are known to provide the energy required to overcome the energy barrier for membrane fusion. WEBER et $a l$. were the first who proved that a minimalistic machinery, comprised of the three neuronal SNAREs syntaxin-1A, SNAP25 and synaptobrevin 2, is capable to induce membrane fusion in vitro. ${ }^{[19]}$ This was the starting point for a multitude of different in vitro approaches to study SNARE-mediated membrane fusion. However, fusion kinetics observed in the majority of these in vitro assays were determined to be slower by several orders of magnitude compared to the fusion rates found in synapses and neuroendocrine cells. This discrepancies between in vivo and in vitro experiments can be attributed to the simplicity of most in vitro approaches, concerning spatial and temporal organization of the SNAREs as well as lacking important regulative proteins, lipids, or other cofactors. Beyond that, the $\mathrm{Ca}^{2+}$-triggered dramatic acceleration of synaptic vesicle fusion, as it is observed in vivo, was not sufficiently reproduced in vitro until now and the exact mechanism remains enigmatic. Only the role of synaptotagmin-1 (syt-1) as the main candidate for a $\mathrm{Ca}^{2+}$-sensor in neuronal exocytosis is widely agreed upon. ${ }^{[69],[15],[89]}$

A versatile model system to monitor single vesicle fusion in vitro is based on pore-spanning membranes (PSMs). As PSMs consist of supported as well as free-standing lipid bilayer parts with planar geometry, they resemble the properties of a presynaptic membrane quite well. In this work, they were used to investigate the impact of the phosphoinositide $\mathrm{PI}(4,5) \mathrm{P}_{2}$ on SNARE-mediated fusion, as it was shown that $\mathrm{PI}(4,5) \mathrm{P}_{2}$ localizes to hot spots of synaptic vesicle exocytosis in neuroendocrine $\mathrm{PC} 12$ cells and is thought to preorganize the t-SNAREs. ${ }^{[51]}{ }^{[16]}$ Further, the model system was used to analyze the influence of syt-1 on SNARE-mediated fusion in the absence and presence of $\mathrm{Ca}^{2+}$, to gain a deeper understanding of the $\mathrm{Ca}^{2+}$-trigger in neuronal exocytosis.

In the last part of the work, the fusion and diffusion behavior of natural secretory chromaffin granules with and on PSMs was in the focus of investigation. 



\section{Materials and Methods}

\subsection{Materials}

\subsubsection{Porous substrates}

For the preparation of pore-spanning membranes (PSMs), porous substrates made of silicon nitride were used as the basic grid. They were purchased from fluXXion B. V. (Eindhoven, The Netherlands) and Aquamarijn B. V. (Zutphen, The Netherlands). These substrates, produced in a photolithographic process, with a square area of $5 \times 5 \mathrm{~mm}^{2}$, had a porous part of $800 \mathrm{~nm}$ thickness in the center surrounded by a $1 \mathrm{~mm}$ wide supporting frame. In this work, substrates with pore diameters of $1.2 \mu \mathrm{m}$ (Figure 3-1A) and $5 \mu \mathrm{m}$ (Figure 3-1B) were used. The pores were arranged in a hexagonal lattice with a surface porosity of $35-40 \%$.

A

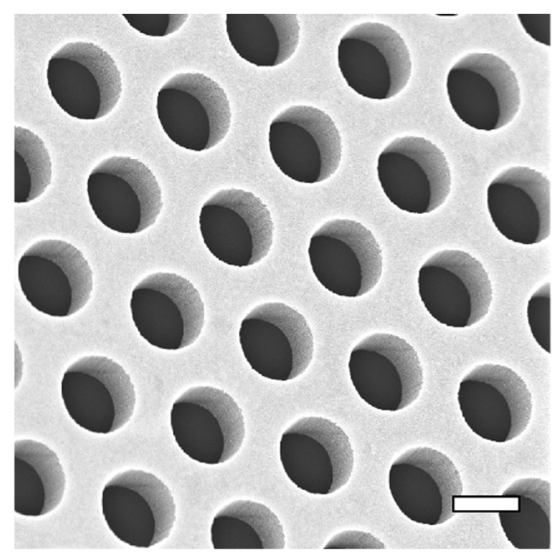

B

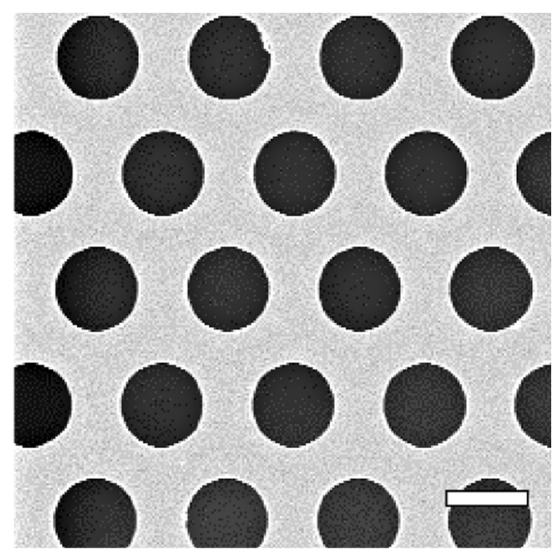

Figure 3-1 Scanning electron micrographs of porous silicon nitride substrates with hexagonal arrangement. (A) Substrate with a pore diameter of $1.2 \mu \mathrm{m}$. Scale bar: $1 \mu \mathrm{m}$. (B) Substrate with a pore diameter of $5 \mu \mathrm{m}$. Scale bar: $5 \mu \mathrm{m} .{ }^{\text {990] }}$

\subsubsection{Lipids}

The lipids to prepare PSMs as well as proteoliposomes were chosen to roughly mimic the lipid composition of synaptic plasma membranes and synaptic vesicles. ${ }^{[91]}{ }^{[92]}$ The basic lipid mixture used for the preparation of PSMs was composed of DOPC/POPE/POPS/PI(4,5) $\mathrm{P}_{2} /$ cholesterol (48:20:10:2:20), whereas the amounts of $\mathrm{PI}(4,5) \mathrm{P}_{2}$ and DOPC were varied depending on the focus of the experiment. For proteoliposome preparation, a standard mixture of DOPC/POPE/POPS/cholesterol (50:20:10:20) was used for the experiments without synaptotagmin-1 (syt-1). For the experiments with syt-1, the amount of POPS was reduced to 5 mol\% while the amount of DOPC was increased to $55 \mathrm{~mol} \%$. An overview of the chemical structures 
and molecular weights for cholesterol and all phospholipids used, excepting the fluorescently labeled ones, is compiled in Table 3-1.

Table 3-1 Chemical structures and molecular weights of cholesterol and phospholipids used in this work.

\section{Lipid}

Molecular weight

cholesterol

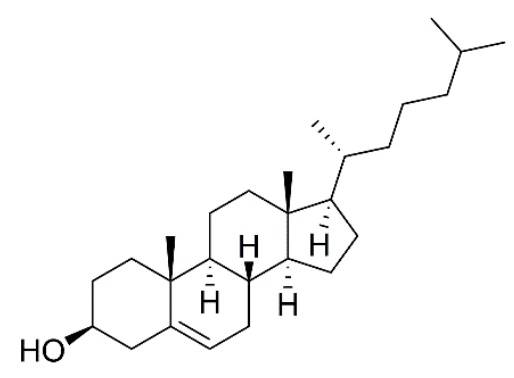

$386.7 \mathrm{~g} / \mathrm{mol}$

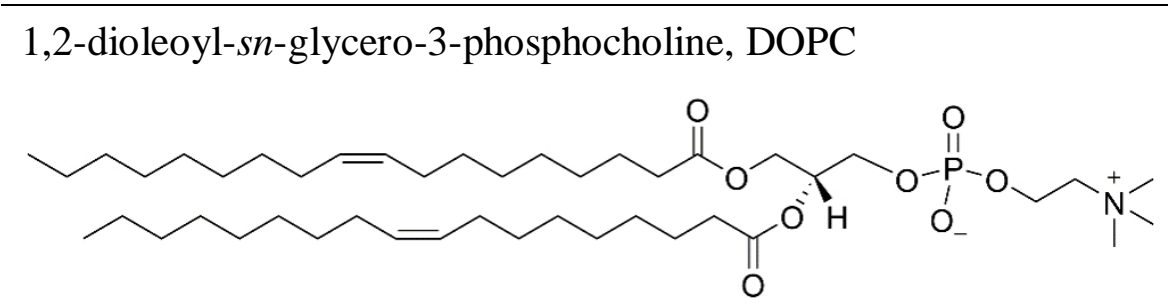

$786.1 \mathrm{~g} / \mathrm{mol}$

1-palmitoyl-2-oleoyl-sn-glycero-3-phospho-L-serine, POPS<smiles>CCCCCCCCCCCCCCCCCCCCCCCC(=O)OC[C@H](O)COP(=O)(O[NH3+])OCC([NH3+])C(=O)[O-]</smiles>

$784.0 \mathrm{~g} / \mathrm{mol}$

1-palmitoyl-2-oleoyl-sn-glycero-3-phosphoethanolamine, POPE

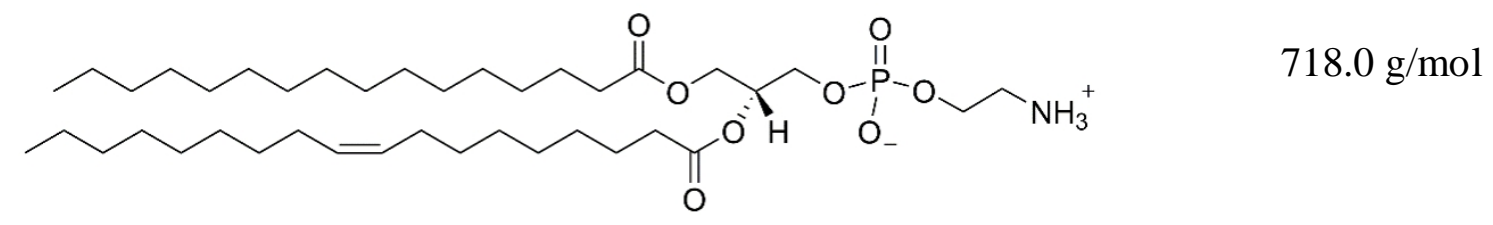

1,2-dipalmitoyl-sn-glycero-3-phosphatidylinositol-4,5-bisphosphate,

$\mathrm{PI}(4,5) \mathrm{P}_{2}$

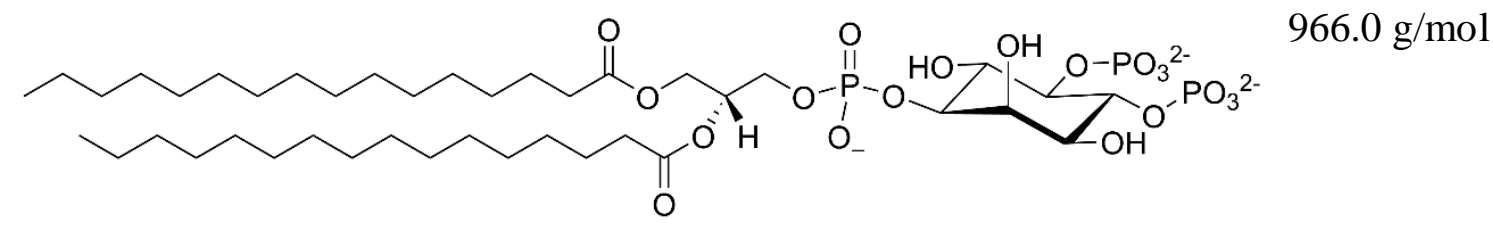




\subsubsection{Fluorescence probes}

The main method used in this work for the investigation of SNARE-mediated single vesicle fusion was confocal laser scanning fluorescence microscopy. For this purpose, proteoliposomes, PSMs as well as the natural secretory vesicles, the chromaffin granules (CGs), had to be doped with fluorescent probes. Artificial membranes - PSMs and proteoliposomes were labeled with the lipid coupled fluorescent dyes Atto488-DPPE and TexasRed-DPPE, respectively, whereas the CGs were doped with the lipophilic fluorescent marker DiD-C 18 . For content labeling of artificial proteoliposomes the water soluble dye sulforhodamine B was used. The chemical structures as well as the maxima for the absorption and emission wavelengths are shown in Table 3-2.

Table 3-2 Chemical structures as well as wavelengths for maximal absorption $\left(\boldsymbol{\lambda}_{\mathbf{a b s}}^{\max }\right)$ and emission ( $\left.\boldsymbol{\lambda}_{\mathbf{e m}}^{\max }\right)$ of fluorescent probes used in this work.

\begin{tabular}{lll}
\hline Fluorescent probe & $\lambda_{\mathrm{abs}}^{\max }$ & $\lambda_{\mathrm{em}}^{\max }$ \\
\hline
\end{tabular}

TexasRed-1,2-dipalmitoyl-sn-glycero-3-phosphoethanolamine,

TxR-DPPE, $M=1382 \mathrm{~g} / \mathrm{mol}$

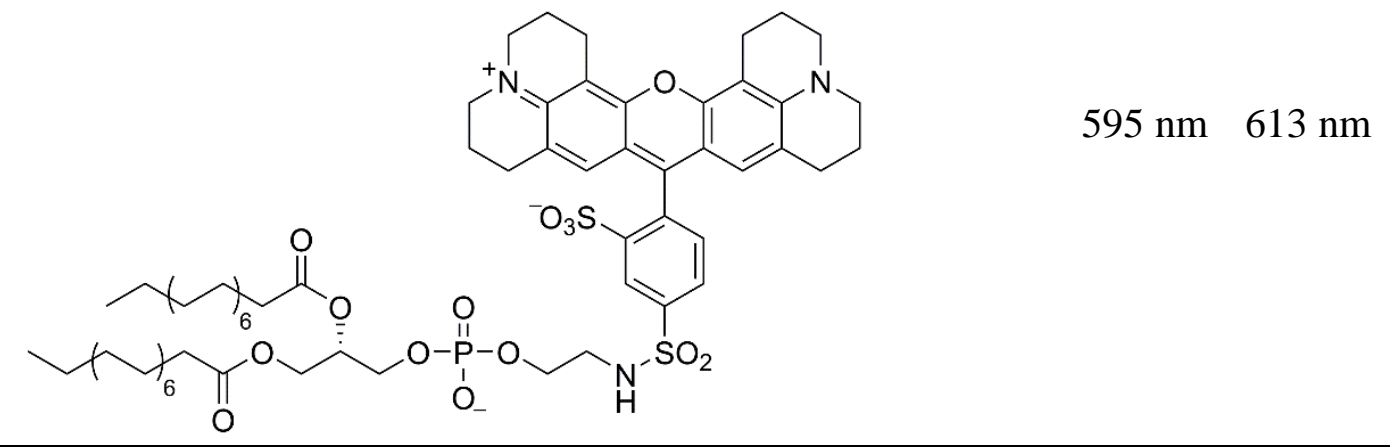

Atto488-1,2-dipalmitoyl-sn-glycero-3-phosphoethanolamine,

Atto488-DPPE, $M=1263 \mathrm{~g} / \mathrm{mol}$

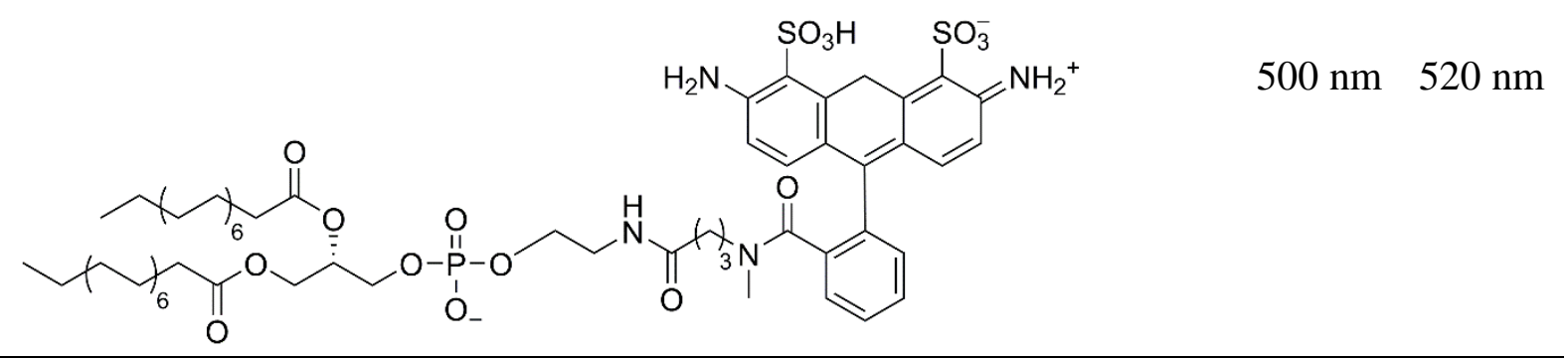


2-((1E,3E)-5-((Z)-3,3-dimethyl-1-octadecylindolin-2-ylidene)penta-1,3-

dien-1-yl)-3,3-dimethyl-1-octadecyl-3H-indol-1-ium perchlorate,

DiD-C $18, M=960 \mathrm{~g} / \mathrm{mol}$

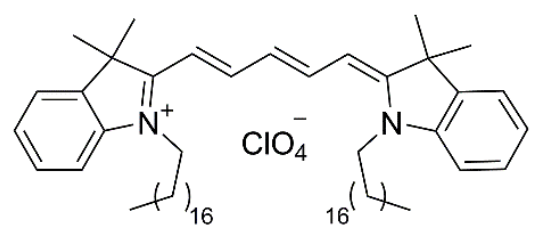

$649 \mathrm{~nm} \quad 667 \mathrm{~nm}$

2-(6-(diethylamino)-3-(diethyliminio)-3H-xanthen-9-yl)-5-

sulfobenzenesulfonate, Sulforhodamine B, $M=559 \mathrm{~g} / \mathrm{mol}$

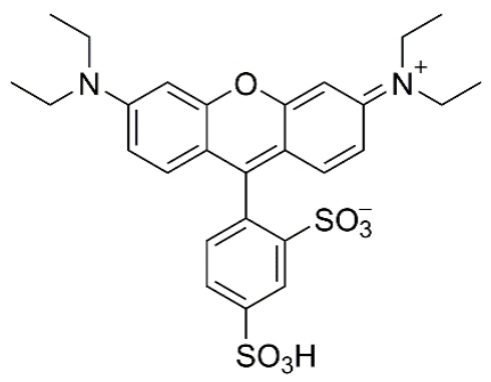

$564 \mathrm{~nm} 582 \mathrm{~nm}$

\subsubsection{SNARE-proteins}

Neuronal SNARE-proteins from the species rattus norvegicus were used as a fusion machinery. They can be subdivided in classes of target membrane SNAREs (t-SNARE) and vesicular SNAREs (v-SNAREs). In a chemical synapse, t-SNAREs are localized in the presynaptic membrane, called target membrane, whereas v-SNAREs are integrated into the membrane of synaptic vesicles.

To induce membrane fusion in our artificial system (cf. Chapter 1.2.2), the v-SNARE synaptobrevin 2 (residues 1-116, syb 2) and a modified t-SNARE complex, the $\Delta$ N49-complex, were utilized. $\triangle \mathrm{N} 49$ is a t-SNARE acceptor complex comprised of the two t-SNAREs SNAP25a (residues 1-206) and syntaxin-1A (residues 183-288, syx-1A) which is stabilized by the binding of a short fragment of the syb 2 SNARE domain (residues 49-96). This complex (Figure 3-2B) was developed by РоBBATI et al. and was shown to enhance the kinetics of in vitro SNARE-mediated fusion drastically in comparison to monomeric t-SNAREs. ${ }^{[21]}$ From their study, it was concluded that partial binding of syb 2 (49-96) to a 1:1 complex of syx-1A and SNAP25a prevents the binding of a second syx-1A, which would lead to the formation of a fusion inhibiting 2:1 complex. Whereas syx-1A is not able to bind to $\Delta \mathrm{N} 49$, full length syb 2 is. It can bind to the unoccupied $N$-terminal part of the SNARE-motif to displace the short fragment and induce fusion by formation of a highly stable tetrameric SNARE-bundle (Figure 
3-2A). For control experiments, designed to assure SNARE specific fusion, $\Delta$ N49 was blocked by the addition of syb 2 (residues 1-96) prior to fusion experiments. This syb 2 variant is comprised of the full SNARE-domain of syb 2 lacking the $C$-terminal transmembrane part.

A

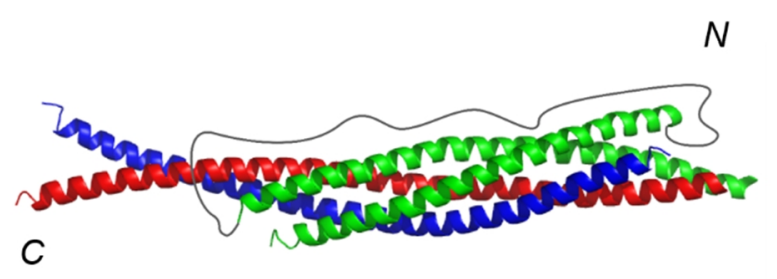

B

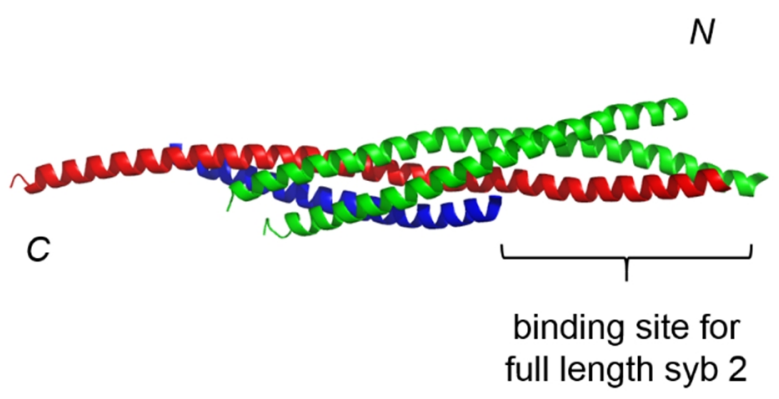

Figure 3-2 (A) Ribbon plot of the tetrameric bundle of neuronal SNAREs comprised of syx-1A (residues 183-288, red), SNAP25a (residues 1-206, green) and syb 2 (residues 1-116, blue), derived from X-ray diffraction.[10] (B) Ribbon plot of the t-SNARE acceptor complex $\Delta$ N49 made up by syx-1A (residues 183288, red), SNAP25a (residues 1-206, green) and a fragment of syb 2 (residues 49-96, blue). In this complex the N-terminal part is unoccupied and allows for binding of full length syb 2 (residues 1-116) to displace the syb 2 fragment.[21] [90]

\section{SNAP25a}

In a chemical synapse, the t-SNARE SNAP25a is anchored in the presynaptic membrane via four palmitoyl linkers, which are posttranslationally connected to cysteines at positions 85,88 , 90 and 92 in the amino acid (aa) sequence (cf. Chapter 7.1.3). To exclude side reactions during heterologous expression of SNAP25a in E. coli and to prevent cross linking, a mutant was used in which the four cysteines were replaced by alanine. Values for important properties of SNAP25a are listed in Table 3-3.

Table 3-3 Values for molecular mass (M), isoelectric point (pI) and extinction coefficient ( $\varepsilon$ ) of SNAP25a.

\begin{tabular}{lccc}
\hline SNAP25a & $\mathbf{M} / \mathbf{k D a}$ & $\mathbf{p I}$ & $\boldsymbol{\varepsilon} / \mathbf{M}^{\mathbf{- 1}} \cdot \mathbf{c m}^{-\mathbf{1}}$ \\
\hline (residues 1-206) & 23.186 & 4.66 & 7240 \\
\hline
\end{tabular}

SNAP25a has a total length of 206 amino acids and exhibits two $\alpha$-helical parts (residues 7-82 and residues 142-201) harboring the two SNARE-motifs that are involved in the formation of the parallel four helix SNARE-bundle (Figure 3-2A). A schematic drawing of the secondary structure of SNAP25a, highlighting all parts of interest, is depicted in Figure 3-3. 


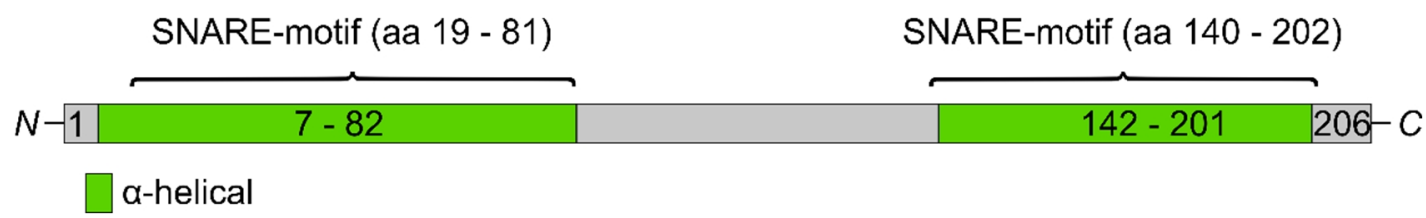

Figure 3-3 Schematic picture of the secondary structure of SNAP25a (residues 1-206). The $\alpha$-helical parts are highlighted in green, whereas unstructured parts are depicted in grey.[93]

\section{Syntaxin-1A}

The t-SNARE syntaxin-1A (syx-1A) was used in a shortened version, lacking the $N$-terminal $\mathrm{H}_{\mathrm{abc}}$-domain, which constitutes a substantial part of the cytosolic region of the protein. The $\mathrm{H}_{\mathrm{abc}}$-domain was shown to regulate opening and closing of the t-SNARE under physiological conditions. ${ }^{[94]}[95]$ To control open and closed states, regulatory factors and proteins like munc-18 are needed. So, for simplification and enhancement of fusiogenity in our model system, the mutant syx-1A (residues 183-288) was used comprising the SNARE-motif (residues 192-254) and the transmembrane domain (TMD, residues 266-288) as main parts of the protein. Values for important characteristics of this mutant are listed in Table 3-4.

Table 3-4 Values for molecular mass (M), isoelectric point (pI) and extinction coefficient $(\varepsilon)$ of syx-1A.

\begin{tabular}{lccc}
\hline syx-1A & M/ kDa & pI & $\varepsilon / \mathbf{M}^{-\mathbf{1}} \cdot \mathbf{c m}^{-\mathbf{1}}$ \\
\hline (residues 183-288) & 12.035 & 5.90 & 4470 \\
\hline
\end{tabular}

A schematic drawing of the secondary structure of syx-1A (183-288) is shown in Figure 3-4. The $\alpha$-helical parts (residues 192-254 and residues 261-284) in the aa sequence (highlighted in green) clearly overlap with the functional motifs (SNARE-motif and TMD) of the protein.

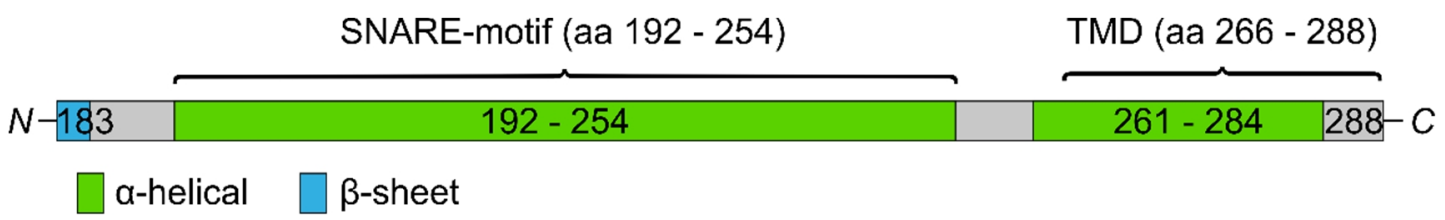

Figure 3-4 Schematic picture of the secondary structure of syntaxin-1A (residues 183-288). The $\alpha$-helical parts are highlighted in green and the $\beta$-sheets in blue, whereas unstructured parts are depicted in grey.[96] 


\section{Synaptobrevin 2}

Synaptobrevin 2 (residues 1-116, syb 2), also referred to as full length syb 2, is a SNAREprotein localized in vivo in the membrane of synaptic vesicles and, therefore, belongs to the class of v-SNAREs. Syb 2 exhibits a $C$-terminal transmembrane domain (TMD, residues 95114) with $\alpha$-helical character serving as a membrane anchor. The SNARE-motif (residues 3191), also showing an $\alpha$-helical secondary structure is connected to the TMD via a short linker region (Figure 3-5).

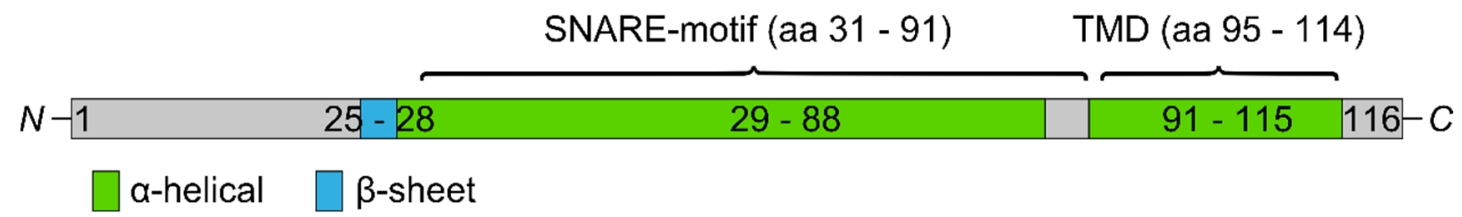

Figure 3-5 Schematic picture of the secondary structure of synaptobrevin 2 (residues 1-116). The $\alpha$-helical parts are highlighted in green and the $\beta$-sheets in blue, whereas unstructured parts are depicted in grey. ${ }^{[97]}$

Besides the full length syb 2, also shortened versions of the protein were used. So, for formation of the stabilized t-SNARE acceptor complex $\Delta \mathrm{N} 49$ the fragment syb 2 (residues 49-96) was needed and for control experiments to verify SNARE specificity, the water soluble fragment syb 2 (residues 1-96) carrying the full SNARE-motif was utilized. Table 3-5 compiles values for important characteristics of all variants in length of syb 2.

Table 3-5 Values for molecular mass (M), isoelectric point (pI) and extinction coefficient $(\varepsilon)$ of all variants in length of syb 2.

\begin{tabular}{lccc}
\hline syb 2 & $\mathbf{M} / \mathbf{k D a}$ & $\mathbf{p I}$ & $\boldsymbol{\varepsilon} / \mathbf{M}^{\mathbf{- 1}} \cdot \mathbf{c m}^{\mathbf{- 1}}$ \\
\hline (residues 1-116) & 12.691 & 7.84 & 13980 \\
(residues 1-96) & 10.517 & 8.04 & 12660 \\
(residues 49-96) & 5.627 & 9.16 & 12490 \\
\hline
\end{tabular}

\subsubsection{Synaptotagmin-1}

Synaptotagmin-1 (residues 1-421, syt-1) is one of the main candidates as a $\mathrm{Ca}^{2+}{ }_{- \text {sensor in }}$ neuronal signal transmission. It is comprised of a short and unstructured intravesicular part (residues 1-57) at the $N$-terminus, followed by a single $\alpha$-helical transmembrane domain (TMD, residues 58-79) and a cytosolic part (residues 80-421). An unstructured linker region in the cytosolic part (residues 80-142) connects the TMD with the two $\mathrm{Ca}^{2+}$ binding domains $\mathrm{C} 2 \mathrm{~A}$ 
(residues 143-244) and C2B (residues 274-377). ${ }^{[98]}$ A schematic drawing, which illustrates the arrangement of the aforementioned domains, is shown in Figure 3-6.

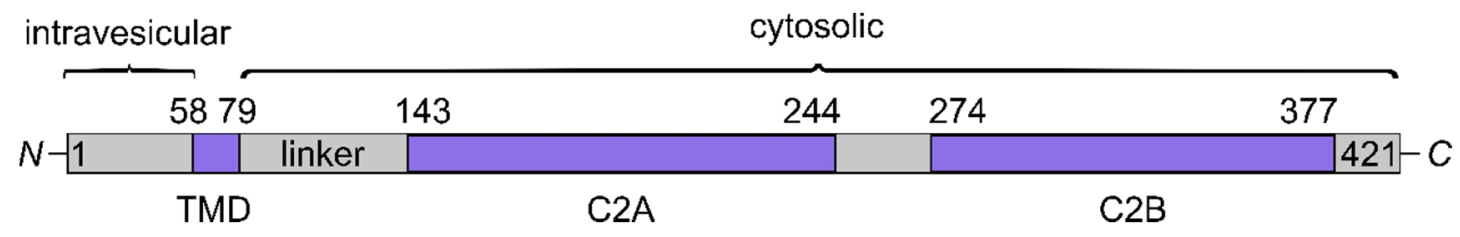

Figure 3-6 Schematic display of the arrangement of important domains of syt-1. The transmembrane domain (TMD, residues 58-79) splits the protein into the unstructured intravesicular part (residues 1-57) and the cytosolic part (residues 80-421). The cytosolic part harbors the two $\mathrm{Ca}^{2+-b i n d i n g ~ d o m a i n s ~} \mathrm{C} 2 \mathrm{~A}$ (residues 143-244) and C2B (residues 274-377).[98]

Characteristic properties of syt-1 are listed in Table 3-6 and the aa sequence in Chapter 7.1.4.

Table 3-6 Values for molecular mass (M), isoelectric point (pI) and extinction coefficient ( $\varepsilon$ ) of syt-1.

\begin{tabular}{lccc}
\hline syt-1 & M/ kDa & pI & $\varepsilon / \mathbf{M}^{\mathbf{- 1} \cdot \mathbf{c m}^{\mathbf{- 1}}}$ \\
\hline (residues 1-421) & 47.4 & 8.57 & 38390 \\
\hline
\end{tabular}




\subsection{Preparative methods}

\subsubsection{SDS-polyacrylamide gel electrophoresis (SDS-PAGE)}

Electrophoresis describes a procedure to separate macromolecules in an electric field. A common technique for separating peptides and proteins by electrophoresis uses a discontinuous polyacrylamide gel as a support medium and sodium dodecyl sulfate (SDS) for denaturation and electrical masking. This method is called sodium dodecyl sulfate polyacrylamide gel electrophoresis (SDS-PAGE) and is a convenient procedure to separate mixtures of proteins depending on their molecular weight. For this purpose, proteins are mixed with the anionic detergent SDS leading to denaturation of the proteins and a net negative charge with a constant charge-to-mass ratio. Applying a voltage across the gel causes the proteins to move towards the anode with a migration speed in the electric field which is only a function of their molecular weight.

In this work, Tricine-SDS-PAGE analysis was performed - a variant developed by SCHÄGGER leading to a better resolution for proteins with a molecular weight smaller than $30 \mathrm{kDa} .^{\text {[99] [100] }}$ This modified version of SDS-PAGE is based on a Tricine-Tris buffer system and a discontinuous gel that consists of a stacking and a separating partition. The compositions of buffers and gels that were used are listed in Table 3-7 and Table 3-8. For the preparation of a $10 \%$ acrylamide gel, $3.4 \mathrm{~mL}$ of the separating gel mixture was transferred to a casting chamber (Mini-PROTEAN Multi-Casting Chamber, Bio Rad, CA, USA) and carefully overlayed with $1 \mathrm{~mL}$ of the stacking gel mixture. The gel was allowed to polymerize for at least $2 \mathrm{~h}$ before being used or stored at $4{ }^{\circ} \mathrm{C}$ for maximum 3 weeks.

Table 3-7 Buffer compositions used for SDS-PAGE analysis.

\section{Composition}

Cathode buffer (10x)

Anode buffer (10x)

Gel buffer (3x)

Sample buffer(2x)

Acrylamide solution
Tris $(1 \mathrm{M})$, Tricine $(1 \mathrm{M}), \mathrm{SDS}(1 \%(w / v)), \mathrm{pH} \sim 8.25$

Tris (2 M), $\mathrm{HCl}(225 \mathrm{mM}), \mathrm{pH} 8.9$

Tris $(3 \mathrm{M}), \mathrm{HCl}(1 \mathrm{M}), \mathrm{SDS}(0.3 \%(w / v)), \mathrm{pH} 8.45$

Tris $(125 \mathrm{~mm})$, SDS $(5 \%(w / v))$, Glycerin $(15 \%$ $(w / v))$, DTT $(0.3 \mathrm{mM})$, Bromophenol blue $(0.02 \%$ $(w / v)), \mathrm{pH} 6.8$

Acrylamide/Bisacrylamide (29:1) (30 \%) 
Table 3-8 Composition and volume fractions for the preparation of four $10 \%$ acrylamide gels.

\begin{tabular}{lcc}
\hline & Stacking gel & Separating gel \\
\hline Acrylamide & $600 \mu \mathrm{L}$ & $5000 \mu \mathrm{L}$ \\
Gel buffer & $1125 \mu \mathrm{L}$ & $3180 \mu \mathrm{L}$ \\
$\mathbf{H}_{\mathbf{2}} \mathbf{O}$ & $2775 \mu \mathrm{L}$ & $1710 \mu \mathrm{L}$ \\
Glycerin (50 \% (w/v)) & -- & $3180 \mu \mathrm{L}$ \\
APS (10\%(w/v)) & $30 \mu \mathrm{L}$ & $75 \mu \mathrm{L}$ \\
TEMED & $6 \mu \mathrm{L}$ & $9 \mu \mathrm{L}$ \\
\hline
\end{tabular}

For SDS-PAGE analysis, the protein samples $(0.5-5 \mu \mathrm{L})$ were mixed with an equivalent volume of sample (2x) buffer and loaded onto the gel, which was installed in an electrophoresis chamber (Mini-PROTEAN Tetra Vertical Electrophoresis Cell, Bio Rad, CA, USA) beforehand. Anode and cathode buffers were added with amounts as stated on the chambers and a voltage of $70 \mathrm{~V}$ was applied across the gel. After $20 \mathrm{~min}$, the voltage was increased to $200 \mathrm{~V}$ until the bromophenol blue front reached the border of the gel. The gel was removed from the chamber, washed by addition of distilled water $(150 \mathrm{~mL})$, exposed to microwave radiation (30-45 s, $800 \mathrm{~W}$ ) and placed on a shaker for $2 \mathrm{~min}$. The procedure was repeated twice, using fresh water for each washing step. For staining, $100 \mathrm{~mL}$ Coomassie G-250 solution ( $80 \mathrm{mg} / \mathrm{mL}$ Coomassie $\mathrm{G}-250,3 \mathrm{~mL}$ conc. $\mathrm{HCl}$ ) was added to the gel, followed by microwaving for $\sim 30 \mathrm{~s}$ at $800 \mathrm{~W}$. After $15 \mathrm{~min}$ of incubation on a shaker, the staining solution was discarded and the gel was rinsed thoroughly with distilled water to remove residual staining solution. To further destain the background, $150 \mathrm{~mL}$ of distilled water was added and incubated for at least $2 \mathrm{~h}$ on a shaker.

\subsubsection{Ion exchange chromatography}

Ion exchange chromatography (IEC) is a method to separate biomolecules according to differences in their net surface charge. Today, IEC is one of the most frequently used techniques for protein purification. It takes advantage of the unique relationship between $\mathrm{pH}$ and net surface charge for a specific protein and utilizes it for separation. IEC is based on the reversible interactions between charged proteins and an oppositely charged, stationary ion exchange matrix. These interactions can be controlled in order to favor binding or elution of specific 
proteins to achieve separation. At the $\mathrm{pH}$ equivalent to its isoelectric point (pI), a protein has no net charge and will not interact with a charged matrix. However, at a pH above its pI, a protein will bind to a positively charged matrix (anion exchanger) and, at a pH below its $\mathrm{pI}$, a protein will bind to a negatively charged matrix (cation exchanger). In addition to the ionic interactions based on COULOMB's law, other types of binding - like VAN DER WAALS forces may occur but are negligible. So, by choosing the right matrix and controlling the ionic strength of the mobile phase, proteins can be separated and concentrated using IEC. The ionic strength of the mobile phase can be adjusted by buffer compositions and applied gradients, starting with low ionic concentrations and moving towards higher ones.

IEC was always carried out at $4{ }^{\circ} \mathrm{C}$ and all used buffers had a $\mathrm{pH}$ value of 7.4. Therefore, the SNARE-protein SNAP25a as well as the t-SNARE acceptor complex $\triangle$ N49 were purified on a MonoQ anion exchange column, whereas the purification of synaptotagmin-1 was performed using a MonoS cation exchanger. Before the purification, using the ÄKTApurifier 10 FPLC (GE Healthcare, Little Chalfont, UK), all protein samples were diluted with low salt ÄKTA buffer A below their elution conductivity and sterilely filtered. The ion exchange matrix was equilibrated in ÄKTA buffer A and the protein sample was loaded onto the column. A buffer gradient composed of ÄKTA buffers A and B was pumped through the column to elute the proteins separately. For each protein, the buffer gradient of A and B was adapted individually as shown in the respective chromatograms. The purification elutions were analyzed in real time by an UV-vis detector $(\lambda=280 \mathrm{~nm})$ and fractionated with an auto sampler (Frac-920, GE Healthcare, Little Chalfont, UK). The collected fractions were subsequently analyzed by SDSPAGE to verify successful protein purification.

\subsubsection{Isolation and purification of SNARE-proteins}

For all SNARE-proteins, DNA sequences, originating from rattus norvegicus, were inserted into a pET28a(+) vector and heterologously expressed in E. coli (BL21 (DE3)). The pET28a(+) vectors containing the individual SNARE sequences were kindly provided by the group of REINHARD JAHN (Max-Plack-Institut für biophysikalische Chemie, Göttingen). Each SNARE was provided with a hexahistidine-tag (His6-tag) to facilitate segregation from other proteins by $\mathrm{Ni}^{2+}$-affinity chromatography in the first step of purification. The His ${ }_{6}$-tag was subsequently cut off by thrombin digestion, followed by the final purification and concentration step of the respective SNARE using ion exchange chromatography. 
The isolation and purification protocol is broadly similar for all SNAREs and was detailed in former dissertations by SCHWENEN and KUHLMANN. ${ }^{[101]}{ }^{[90]}$ Therefore, it will just be described in general in the following.

The isolation of each SNARE-protein started with the transformation of the specific DNA sequence containing pET28a(+) vector into competent E. coli cells (BL21 (DE3)). Glycerol stocks of these transformed cells were then used to inoculate lysogeny broth (LB)-medium ( $75 \mathrm{~mL}, 30 \mu \mathrm{g} / \mathrm{mL}$ kanamycin sulfate) at $37^{\circ} \mathrm{C}$ overnight as a preculture. The main culture was started on the next day by inoculating terrific broth (TB)-medium $(10 \times 250 \mathrm{~mL}, 30 \mu \mathrm{g} / \mathrm{mL}$ kanamycin sulfate), each with $5 \mathrm{~mL}$ preculture, followed by incubation at $37{ }^{\circ} \mathrm{C}$ until an optical density $O D_{600}=0.85-0.95$ was reached. By addition of IPTG $(c($ IPTG $)=0.25 \mathrm{mM})$, the expression was induced and incubation at $37{ }^{\circ} \mathrm{C}$ continued for $2.5-3 \mathrm{~h}$. The cell suspension was centrifuged for $20 \mathrm{~min}$ at $4500 \mathrm{rpm}$ (Allegra TM X-22R, rotor: SX4250, Beckmann Coulter, Brea, CA, USA) to pellet the cells. The supernatant was discarded and the pellet resuspended in resuspension buffer $(80 \mathrm{~mL})$ to be stored at $-20{ }^{\circ} \mathrm{C}$ at least overnight. The frozen, resuspended cells were defrosted at room temperature and the lysis was started by addition of lysozyme $(10 \mathrm{mg}), \mathrm{MgCl}_{2}\left(c\left(\mathrm{MgCl}_{2}\right)=1 \mathrm{mM}\right)$, DNase I $(2 \mathrm{mg})$ and one tablet of cOmplete (EDTA-free) for protease inhibition. The resulting suspension was stirred for $30 \mathrm{~min}$ at room temperature, homogenized with a Teflon potter and passed through a microfluidizer (Microfluidizer LM10, Microfluidics Corp., MA, USA) for three cycles at 1000 bar for efficient disruption of the cells. The lysate was filled up with extraction buffer $(1: 1(v / v))$ and a final concentration of $6 \mathrm{M}$ urea was adjusted prior to centrifugation (3K30, rotor: 19776, Sigma, Osterode am Harz, Germany) for $1 \mathrm{~h}$ at $14000 \mathrm{rpm}$ and $4{ }^{\circ} \mathrm{C}$. The supernatant was mixed with $\mathrm{Ni}^{2+}$-agarose beads $(8-12 \mathrm{~mL})$ and incubated on a roller mixer for $2.5 \mathrm{~h}$ at $4{ }^{\circ} \mathrm{C}$. The slurry was filtered using a BioRad Econo-Column $(2.5 \times 10 \mathrm{~cm})$ and the protein loaded beads were washed with washing buffer $(4 \times 50 \mathrm{~mL})$. Fractional elution by adding elution buffer $(3 \times 8-10 \mathrm{~mL})$ and direct supplementation with DTT $(c($ DTT $)=10 \mathrm{mM})$ yielded the desired protein still carrying the His6-tag. After SDS-PAGE analysis of the elution fractions, those with a high protein concentration were combined, thrombin $\left(c^{\prime}(\right.$ thrombin $\left.)=0.15 \mathrm{mg} / \mathrm{mL}\right)$ was added for His6-tag removal and the solution was dialyzed against dialysis buffer $(1 \mathrm{~L})$ overnight at $4{ }^{\circ} \mathrm{C}$. Dialysis tubings with molecular weight cutoffs (MWCO) of 4-6 kDa or 12-14 kDa were used, depending on the size of the particular protein. The cleavage of the His6-tag was verified by SDS-PAGE and, if completed successfully, the protein was further purified and concentrated by ion exchange chromatography (IEC). For IEC, the ÄKTApurifier 10 FPLC (GE Healthcare, Little Chalfont, UK) was used and the collected ÄKTA fractions were analyzed by SDS-PAGE 
to finally combine the fractions with the highest yields and purities. The obtained SDS-PAGE gels, after cell disruption and IEC, as well as the corresponding IEC chromatograms for SNAP25a and $\triangle \mathrm{N} 49$ are shown in the following. The proteins syb 2, in all modifications (residues 49-96, residues 1-96, residues 1-116), and syx-1A (residues 183-288) were purified by former PhD students in our lab LANDO SCHWENEN and JAN KUHLMANN.

\section{SNAP25a}

SDS-PAGE gels after cell disruption and $\mathrm{Ni}^{2+}$-affinity chromatography as well as His6-tag cleavage of an exemplary SNAP25a isolation are shown in Figure 3-7.

A

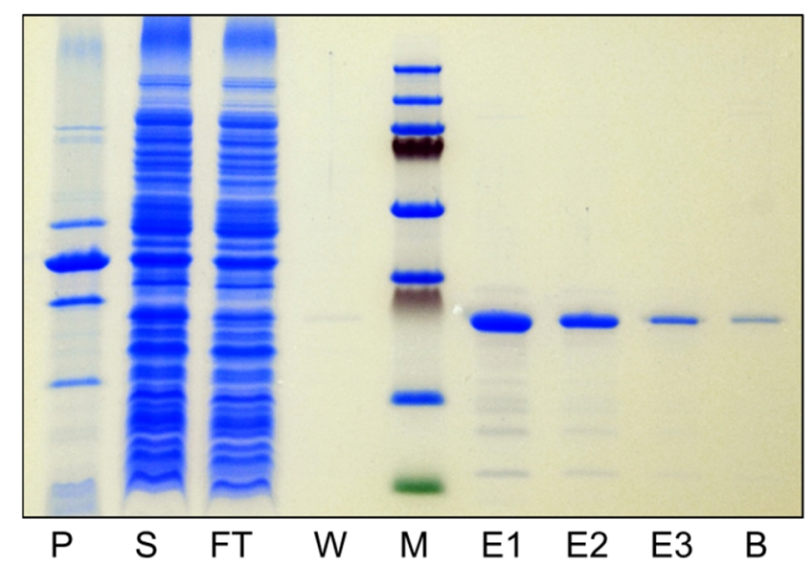

B

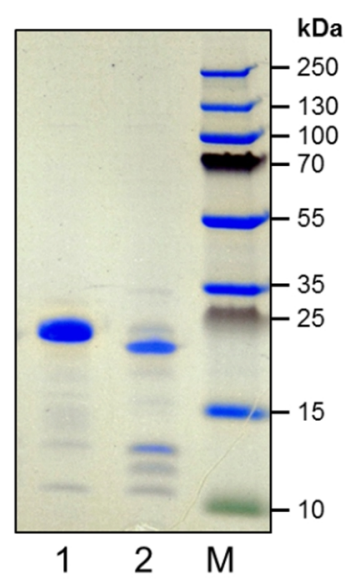

Figure 3-7 SDS-PAGE analysis of an exemplary SNAP25a ( $\mathrm{M}=23.186 \mathrm{kDa})$ isolation. (A) Gel for the control of cell disruption, expression and $\mathrm{Ni}^{2+}$-affinity chromatography (P: pellet, $\mathrm{S}$ : supernatant, FT: flow through, W: wash, M: marker, E1-E3: elution fractions, B: beads). (B) Gel for the control of His6-tag cleavage (1: before cleavage (pooled elution fractions before thrombin addition), 2: after cleavage (pooled elution fractions after thrombin addition and dialysis overnight).

After $\mathrm{Ni}^{2+}$-affinity chromatography (lanes E1-E3 in Figure 3-7A), a dominant band occurs at $\sim 23 \mathrm{kDa}$, which was assigned to SNAP25a - the target protein. Lane 1 in Figure 3-7B represents the combined elution fractions E1-E3 before addition of thrombin to cleave the His 6 -tag. Lane 2 in Figure 3-7B shows the same sample after addition of thrombin and dialysis overnight at $4{ }^{\circ} \mathrm{C}$. A slight shift to a lower molecular weight of the SNAP25a-band in comparison to lane 1 is observable, verifying the successful cleavage of the His 6 -tag.

After the confirmation of successful expression and removal of the His6-tag by SDS-PAGE, SNAP25a was further purified using the strong anion exchange column MonoQ 10/100 (GE Healthcare, Little Chalfont, UK). The resulting ÄKTA chromatogram as well as the SDSPAGE analysis of the elution fractions of interest are depicted in Figure 3-8. 
A

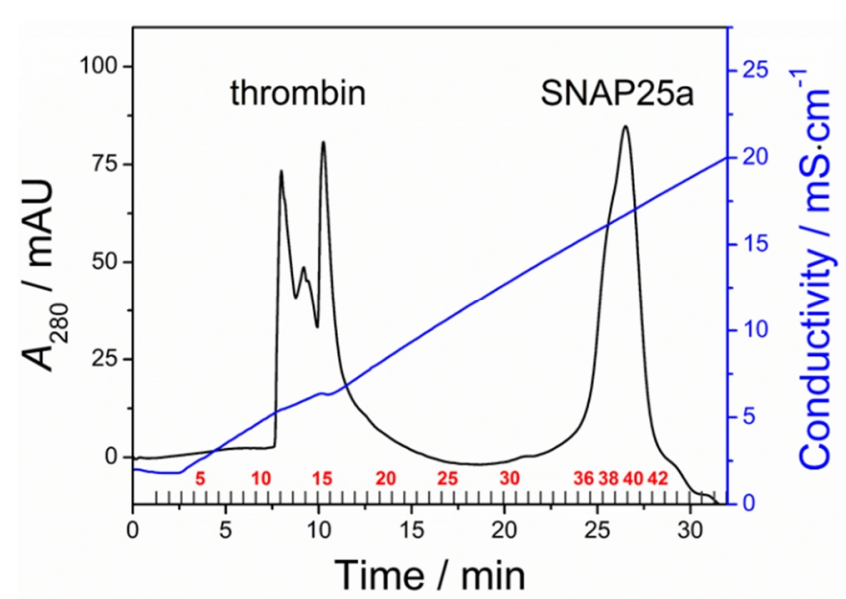

B

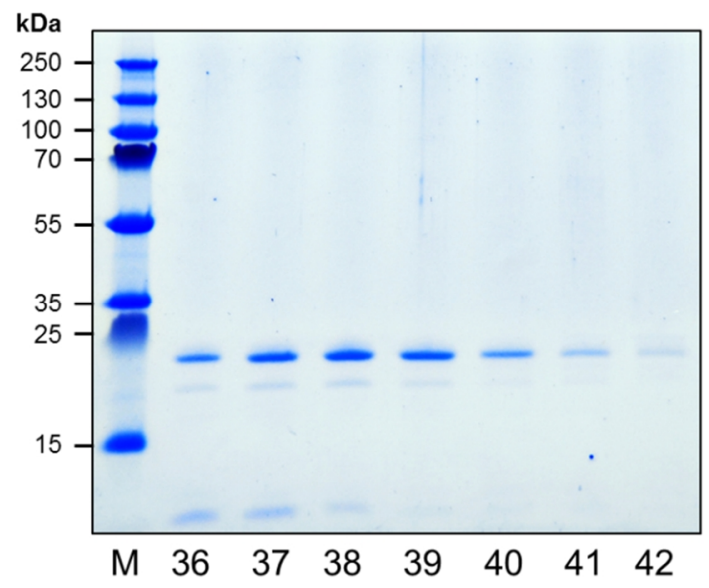

Figure 3-8 (A) Ion exchange chromatogram obtained after purification of SNAP25a on a MonoQ 10/100 column. SNAP25a elutes in a conductivity range between $14.85 \mathrm{mS} / \mathrm{cm}$ and $17.20 \mathrm{mS} / \mathrm{cm}$ (fractions 36-42). (B) SDS-PAGE analysis of the elution fractions 36-42 showing a dominant band at $\sim 23 \mathrm{kDa}$.

The dominant band in the elution fractions $36-40$ at $\sim 23 \mathrm{kDa}$ was assigned to the target SNARE-protein SNAP25a. It elutes in a conductivity range between $14.85 \mathrm{mS} / \mathrm{cm}$ and $17.20 \mathrm{mS} / \mathrm{cm}$ with a maximum value for $A_{280}$ at $16.68 \mathrm{mS} / \mathrm{cm}$ (Figure 3-8A). Fractions 37-40 were combined and the total protein concentration determined by UV-vis spectroscopy using the NanoDrop 2000c (Thermo Fischer Scientific, Waltham, MA, USA). Estimating a calculated extinction coefficient for the sequence of SNAP25a (cf. Chapter 7.1.3) of $\varepsilon=7240 \mathrm{M}^{-1} \cdot \mathrm{cm}^{-1}$, a total of $4 \mathrm{mg}$ SNAP25a was isolated from $2.5 \mathrm{~L}$ main culture.

\section{$\Delta$ N49-complex}

Intensive studies of SNARE-mediated liposome fusion by POBBATI et al. showed that in vitro fusion was significantly enhanced using the $\Delta$ N49-complex $(\Delta N 49)$ as a pre-organized t-SNARE acceptor complex. ${ }^{[21]} \Delta \mathrm{N} 49$ is a preassembled complex of the two t-SNAREs SNAP25a and syx-1A, stabilized by a short fragment of the syb 2 SNARE-domain (residues 49-96). The formation of $\Delta \mathrm{N} 49$ was accomplished by the assembly of syx 1A, SNAP25a and syb 2 (residues 49-96) in a final ratio of 1:2:2. Prior to assembly, SNAP25a and syb 2 (residues 49-96) were supplemented with CHAPS (1 \%, (w/v)). Syx-1A and syb 2 (residues 4996) were pre-assembled for $30 \mathrm{~min}$ at room temperature, then SNAP25a was added and the resulting mixture was incubated overnight at $4{ }^{\circ} \mathrm{C}$. For the subsequent purification and concentration of $\Delta \mathrm{N} 49$ by IEC, the anion exchange column MonoQ 5/50 was used. The obtained ÄKTA chromatogram as well as the corresponding SDS-PAGE analysis of the elution fractions of interest for an exemplary $\Delta \mathrm{N} 49$ formation are shown in Figure 3-9. 
A

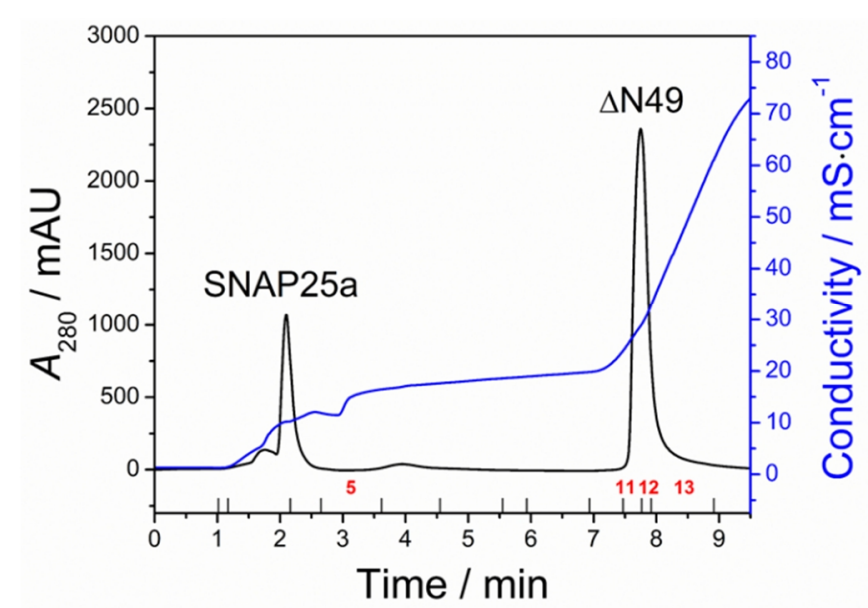

B

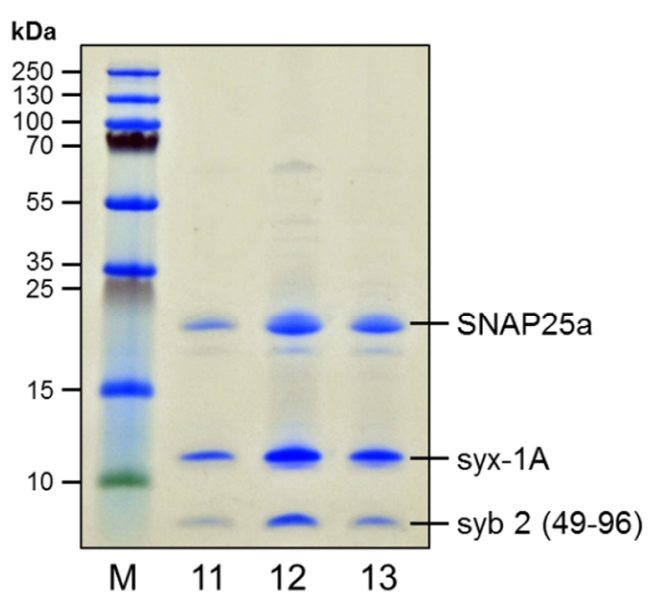

Figure 3-9 (A) Ion exchange chromatogram obtained after purification of $\Delta$ N49 on a MonoQ $5 / 50$ column. $\Delta \mathrm{N} 49$ elutes in a conductivity range between $26.22 \mathrm{mS} / \mathrm{cm}$ and $32.36 \mathrm{mS} / \mathrm{cm}$ (fractions 11-13). (B) SDSPAGE analysis of the elution fractions $11-13$ showing three dominant bands at $\sim 23 \mathrm{kDa}, \sim 12 \mathrm{kDa}$ and $\sim 5 \mathrm{kDa}$ referring to SNAP25a, syx-1A and syb 2 (residues 49-96).

The three dominant bands in the elution fractions $11-13$ at $\sim 23 \mathrm{kDa}, \sim 12 \mathrm{kDa}$ and $\sim 5 \mathrm{kDa}$ (Figure 3-9B) were assigned to the three monomeric SNAREs SNAP25a, syx-1A and syb 2 (49-96) making up the $\Delta \mathrm{N} 49$-complex and therefore, proving its successful formation. $\Delta \mathrm{N} 49$ elutes in a conductivity range between $26.22 \mathrm{mS} / \mathrm{cm}$ and $32.36 \mathrm{mS} / \mathrm{cm}$ with a maximum value for $A_{280}$ at $28.80 \mathrm{mS} / \mathrm{cm}$ (Figure 3-9A). The peak at a retention time of $\sim 2 \mathrm{~min}$, starting to elute at a conductivity of $14.55 \mathrm{mS} / \mathrm{cm}$ (fractions 3-4), was assigned to SNAP25a, which was set in in excess for the complex formation. The excess of syb 2 (49-96) was not observed in the ÄKTA chromatogram. Due to its $\mathrm{pI}$ of 9.16 it did not bind to the anion exchange matrix but immediately passed through during addition of the mixture onto the column.

\subsubsection{Isolation and purification of synaptotagmin-1}

Synaptotagmin-1 (syt-1, residues 1-421) - the main $\mathrm{Ca}^{2+}$ sensor of neuronal exocytosis - was recombinantly expressed using the E. coli strain BL21-CodonPlus (DE3)-RIPL. This specific strain enables efficient high-level expression of heterologous proteins by containing extra copies of the t-RNA genes $\arg U$, ileY, leuW and proL. These additional t-RNA genes prevent a restriction in the translation process by filling the gap generated by certain t-RNAs, which are rare in the $E$. coli host strain but needed for recombinant protein expression.

A glycerol stock of transformed E. coli (BL21-CodonPlus (DE3)-RIPL) cells, carrying a pET-28a(+) vector with the inserted DNA sequence encoding for syt-1, was kindly provided by the group of REINHARD JAHN (Max-Planck-Institute for biophysical chemistry). The DNA- 
sequence was inserted into the multiple cloning site using the restriction enzymes $N d e$ I and $X h o$ I. Due to that, a His6-tag and a thrombin cut sequence were attached to the $N$-terminus of syt-1, facilitating the segregation from other proteins in the lysate by applying $\mathrm{Ni}^{2+}$-affinity chromatography as the first step of purification. The His $6^{-t a g}$ was subsequently cut off by thrombin digestion, followed by the final purification and concentration step using ion exchange chromatography (IEC). Table 3-9 and Table 3-10 show the composition of the media and buffers that were used for the expression of syt-1, which will be detailed in the following.

Table 3-9 Composition of the expression media.

\section{Composition}

\begin{tabular}{ll}
\hline LB-medium & Trypton $(10 \mathrm{~g} / \mathrm{L})$, yeast extract $(5 \mathrm{~g} / \mathrm{L}), \mathrm{NaCl}(5 \mathrm{~g} / \mathrm{L})$ \\
TB-medium & Trypton $(13.5 \mathrm{~g} / \mathrm{L})$, yeast extract $(26.5 \mathrm{~g} / \mathrm{L})$, glycerin $(4.5 \mathrm{~g} / \mathrm{L})$ \\
TB-salt & $\mathrm{K}_{2} \mathrm{HPO}_{4}(125 \mathrm{~g} / \mathrm{L}), \mathrm{KH}_{2} \mathrm{PO}_{4}(23 \mathrm{~g} / \mathrm{L})$ \\
\hline
\end{tabular}

Table 3-10 List of buffer compositions used for syt-1 isolation and purification.

\section{Composition}

Resuspension buffer

Extraction buffer

Wash buffer

Elution buffer

Dialysis buffer

ÄKTA buffer A

ÄKTA buffer B
Tris (20 mM), $\mathrm{NaCl}(500 \mathrm{mM})$, imidazole (8 mM), $\mathrm{pH}=7.4$

Tris (20 mM), $\mathrm{NaCl}(500 \mathrm{mM})$, imidazole $(8 \mathrm{mM})$, sodium cholate $(10 \%(w / v)), \mathrm{pH}=7.4$

Tris (20 mM), $\mathrm{NaCl}(300 \mathrm{~mm})$, imidazole (50 mM), CHAPS $(1 \%(w / v)), \mathrm{pH}=7.4$

Tris (20 mM), $\mathrm{NaCl}$ (300 mM), imidazole (400 mM), CHAPS $(1 \%(w / v))$, DTT $(1 \mathrm{mM})^{*}, \mathrm{pH}=7.4$

Tris (20 mM), $\mathrm{NaCl}$ (300 mM), imidazole (400 mM), CHAPS $(1 \%(w / v))$, EDTA $(1 \mathrm{mM})$, DTT $(1 \mathrm{mM})^{*}, \mathrm{pH}=7.4$

HEPES (20 mM), CHAPS (1 \% ( $w / v))$, EDTA (1 mM), DTT $(1 \mathrm{mM})^{*}, \mathrm{pH}=7.4$

HEPES $(20 \mathrm{mM}), \mathrm{NaCl}(1 \mathrm{M})$, CHAPS $(1 \%(w / v))$, EDTA $(1 \mathrm{mM})$, DTT $(1 \mathrm{mM})^{*}, \mathrm{pH}=7.4$

The isolation protocol for syt-1 is broadly similar to the one carried out for the isolation of SNARE-proteins. From cryo glycerol stocks precultures in LB-medium $(2 \times 75 \mathrm{~mL}$, supplemented with $30 \mu \mathrm{g} / \mathrm{mL}$ kanamycin sulfate) were grown overnight at $37^{\circ} \mathrm{C}$ at $175 \mathrm{rpm}$. 
The main culture was started by inoculation of TB-medium $(10 \times 250 \mathrm{~mL}$, supplemented with $30 \mu \mathrm{g} / \mathrm{mL}$ kanamycin sulfate), each with $5 \mathrm{~mL}$ of the overnight preculture and incubated at $37^{\circ} \mathrm{C}$ and $160 \mathrm{rpm}$ until an optical density of $O D_{600}=0.85-0.95$ was reached. At this point, IPTG $((c($ IPTG $)=0.25 \mathrm{mM})$ was added to start the lac-operon controlled expression of syt-1, followed by further incubation at $37^{\circ} \mathrm{C}$ and $160 \mathrm{rpm}$ for $3 \mathrm{~h}$. To harvest the cells, the medium suspension was centrifuged for $20 \mathrm{~min}$ at $4{ }^{\circ} \mathrm{C}$ and $4500 \mathrm{rpm}$ (Allegra TM X-22R, rotor: SX4250, Beckmann Coulter, Brea, CA, USA). The supernatant was discarded and the pellet resuspended in resuspension buffer $(80 \mathrm{~mL})$ to be stored at $-20{ }^{\circ} \mathrm{C}$ at least overnight. The frozen, resuspended cells were defrosted at room temperature and the lysis was started by addition of lysozyme $(10 \mathrm{mg}), \mathrm{MgCl}_{2}\left(c\left(\mathrm{MgCl}_{2}\right)=1 \mathrm{mM}\right)$, DNase I (2 mg) and one tablet of cOmplete (EDTA-free) for protease inhibition. This suspension was stirred for $30 \mathrm{~min}$ at room temperature, homogenized with a Teflon potter and passed for three cycles through a microfluidizer (Microfluidizer LM10, Microfluidics Corp., MA, USA) at 1000 bar for efficient disruption of the cells. The lysate was filled up with extraction buffer $(1: 1(v / v))$ and stirred for $30 \mathrm{~min}$ at room temperature prior to centrifugation (3K30, rotor: 19776, Sigma, Osterode am Harz, Germany) for $1 \mathrm{~h}$ at $14000 \mathrm{rpm}$ and $4{ }^{\circ} \mathrm{C}$. All following steps were performed at $4{ }^{\circ} \mathrm{C}$. The supernatant was mixed with $\mathrm{Ni}^{2+}$-agarose beads $(10 \mathrm{~mL})$ and incubated on a roller mixer for $3 \mathrm{~h}$. The slurry was transferred to centrifuge tubes and centrifuged for $4 \mathrm{~min}$ at $1000 \mathrm{rpm}$ (Allegra TM X-22R, rotor: SX4250, Beckmann Coulter, Brea, CA, USA). After taking a sample ( $10 \mu \mathrm{L}$, flow through) for SDS-PAGE analysis, the supernatant was discarded and the residual protein loaded beads were resuspended in washing buffer $(10 \mathrm{~mL})$ and incubated for $10 \mathrm{~min}$ on a roller mixer. Again, the suspension was centrifuged for $4 \mathrm{~min}$ at $1000 \mathrm{rpm}$ and a sample from the supernatant (10 $\mu \mathrm{L}$, washing step 1) was taken for SDS-PAGE analysis. This procedure was repeated twice, but with an increased volume of washing buffer $(20 \mathrm{~mL})$. After the third and last step of washing, the suspension of protein loaded beads was not centrifuged but filtered using a BioRad Econo-Column $(2.5 \times 10 \mathrm{~cm})$. Fractional elution by adding elution buffer $(3 \times 10 \mathrm{~mL})$ and direct supplementation with DTT $(c($ DTT $)=10 \mathrm{mM})$ yielded syt-1, still carrying the His6-tag. After SDS-PAGE analysis of the elution fractions, those with a high protein concentration were combined, thrombin $\left(c^{\prime}(\right.$ thrombin $\left.)=0.15 \mathrm{mg} / \mathrm{mL}\right)$ was added for His6-tag removal and the solution was dialyzed against dialysis buffer $(1 \mathrm{~L})$ overnight at $4{ }^{\circ} \mathrm{C}$. Dialysis tubings with a molecular weight cutoff (MWCO) of 12-14 kDa were used. On the next day, the dialyzed solution was further purified by addition of fresh $\mathrm{Ni}^{2+}$-agarose beads $(4 \mathrm{~mL})$ followed by filtration. This step segregated uncut syt-1, still carrying the His 6 -tag, from syt-1, which was successively cleaved by thrombin. The cleavage of the His ${ }_{6}$-tag was verified by 
SDS-PAGE and, if it was completed successfully, the protein was further purified and concentrated by ion exchange chromatography (IEC). IEC of syt-1 was carried out on an ÄKTApurifier 10 FPLC system (GE Healthcare, Little Chalfont, UK) using the strong cation exchange column MonoS 5/50. A gradient of ÄKTA buffer A and B was applied and the collected ÄKTA fractions were analyzed by SDS-PAGE to combine the fractions with the highest purities and yields.

\subsubsection{Reconstitution of SNARE-proteins into liposomes}

Freshly degassed SNARE buffer (20 mM HEPES, $100 \mathrm{mM} \mathrm{KCl,} 1$ mM DTT, 0.1 mM EGTA, $\mathrm{pH}$ 7.4) was used for all preparation steps. DTT was always added freshly after $30 \mathrm{~min}$ of degassing under vacuum and the buffer was stored protected from light. The osmotic concentration was always in average $220 \pm 5 \mathrm{mOsmol} / \mathrm{kg}$.

\section{Preparation of lipid films}

The lipid mixtures that were used for the preparation of proteoliposomes consisted in general of the phospholipids DOPC, POPE, POPS and PI $(4,5) \mathrm{P}_{2}$ plus the sterol cholesterol. The ratios of the named phospholipids and cholesterol were varied depending on the focus of the experiment, they are listed in Table 3-11 and Table 3-12.

Table 3-11 Lipid composition for the preparation of proteoliposomes doped with $\Delta \mathrm{N} 49$, used for the final preparation of pore-spanning membranes (PSMs).

\section{Mixture 1}

\begin{tabular}{ccccccc}
\hline & PI(4,5)P & DOPC & POPE & POPS & Chol & Atto488-DPPE \\
$\mathbf{m o l} \%$ & $0-5$ & $45-50$ & 19 & 10 & 20 & 1 \\
\hline
\end{tabular}

Table 3-12 Lipid composition for the preparation of proteoliposomes doped with syb 2 or with syb 2 and syt-1.

\section{Mixture 2}

\begin{tabular}{lccccc}
\hline & DOPC & POPE & POPS & Chol & TxR-DPPE \\
$\mathbf{m o l} \%$ & $45-50$ & 19 & $5-10$ & 20 & 1 \\
\hline
\end{tabular}

For the preparation of lipid mixtures with a defined composition, stock solutions of the individual lipids were combined. The stock solutions were prepared by dissolving the respective 
lipid in chloroform with an exception of $\mathrm{PI}(4,5) \mathrm{P}_{2}$, which was dissolved in methanol/chloroform/water (2:1:0.8). After combining the defined volumes of each lipid stock solution to achieve the desired molar ratios, the solvents were evaporated by purging with nitrogen gas and heating to $30{ }^{\circ} \mathrm{C}$. To remove any residual solvent, the lipid films were further dried under vacuum for at least $3 \mathrm{~h}$ at $30^{\circ} \mathrm{C}$. All lipid films were prepared with a total mass of $0.5 \mathrm{mg}$. If not used immediately, the obtained lipid films were sealed with Parafilm(M) and stored at $4{ }^{\circ} \mathrm{C}$, protected from light.

\section{Preparation of small proteoliposomes (proteo-SUVs)}

The reconstitution of SNARE-proteins into small unilamellar vesicles (SUVs) was performed in accordance with a protocol developed by HERNANDEZ. ${ }^{[102]}$ That protocol is based on a detergent mediated protein reconstitution forming mixed micelles composed of lipids, detergent and protein in the first step. The subsequent formation of SUVs doped with the applied transmembrane protein out of the mixed micelles suspension was done by rapid dilution and multiple gel filtration steps to efficiently remove the detergent. The following reconstitution protocol is the general procedure used for the insertion of syb 2 and $\triangle \mathrm{N} 49$ into SUVs. For the co-reconstitution of syb 2 and syt-1 into liposomes the protocol had to be changed due to a higher sensitivity for denaturation of syt-1 and is explained separately in Chapter 3.2.7.

The reconstitution protocol started with the solubilization of the lipid film in SNARE-buffer $(50 \mu \mathrm{L})$ containing the detergent $n$-octyl- $\beta$-D-thioglucopyranosid $(n$-OG, $c(n$-OG) $=75 \mathrm{mM})$. Protein solution was added to this mixture adjusting a final protein to lipid ratio of $\mathrm{p} / \mathrm{l}=1: 500$. The resulting suspension of mixed micelles was incubated for $45 \mathrm{~min}$ at $0{ }^{\circ} \mathrm{C}$ and the detergent was removed via rapid dilution and size exclusion chromatography (illustra NAP-10 G25 column, GE Healthcare, Little Chalfont, UK) in SNARE-buffer. A second size exclusion step in MilliQ was performed subsequently to remove salts and residual detergent yielding proteoSUVs in MilliQ which served as the basis for the preparation of large and giant unilamellar proteoliposomes.

\section{Preparation of large proteoliposomes (proteo-LUVs)}

To obtain large unilamellar proteoliposomes (proteo-LUVs), the proteo-SUV suspension was transferred to a $5 \mathrm{~mL}$ round bottom flask and dried overnight at $4{ }^{\circ} \mathrm{C}$ in a desiccator over saturated sodium chloride solution providing a relative humidity of $76 \%$. This high humidity as well as the slow drying process overnight protected the proteins to a certain degree from 
denaturation and resulted in the formation of proteo-lipid multi bilayer stacks (MBSs). These proteo-lipid MBSs were then re-hydrated by incubation in SNARE-buffer $(500 \mu \mathrm{L})$ for at least $30 \mathrm{~min}$ at room temperature resulting in a suspension of multilamellar proteoliposomes. Extrusion (mini extruder, LiposoFast, Avestin) of this suspension through a polycarbonate membrane with a defined pore size yielded large unilamellar proteo-LUVs. For extrusion, polycarbonate membranes with pore diameters of $1000 \mathrm{~nm}$ and $400 \mathrm{~nm}$ were used, leading to mean diameters for the resulting proteo-LUVs of $320 \mathrm{~nm}$ and $240 \mathrm{~nm}^{[90]}$, respectively. The mean diameters of the proteo-LUVs were determined by dynamic light scattering (DLS). A boxplot of the determined mean vesicle sizes prepared using polycarbonate membranes with nominal pore diameters of $\varnothing=1000 \mathrm{~nm}$ is shown in Figure 3-10A. Further, extrusion was carried out using polycarbonate membranes with nominal pore diameters of $\varnothing=400 \mathrm{~nm}$. An intensity-weighted mean diameter distribution of proteoliposomes prepared using the smaller pore sized polycarbonate membranes was measured by JAN KUHLMANN and is depicted in Figure 3-10B.

A

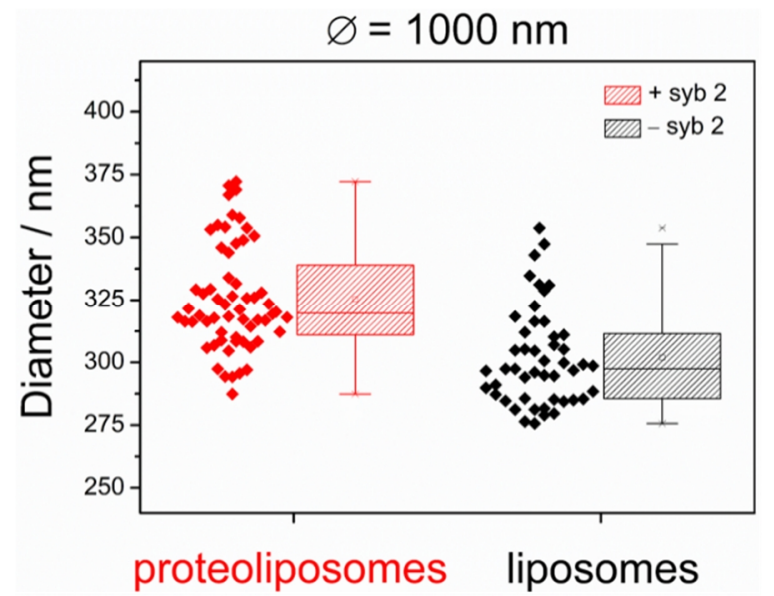

B

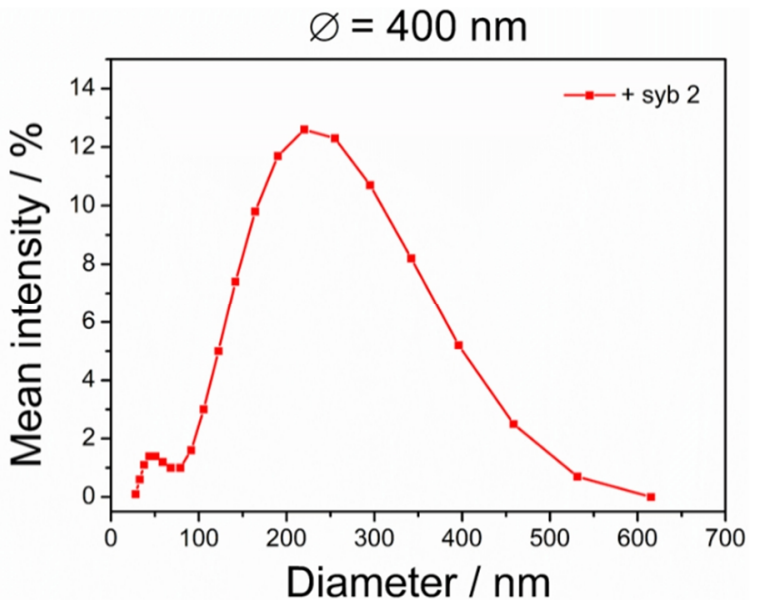

Figure 3-10 DLS-determined vesicle diameters of liposomes and proteoliposomes prepared via extrusion. (A) Boxplot of the determined mean diameters of liposomes (-syb 2) and proteoliposomes (+syb 2) prepared by extrusion through a poly carbonate membrane with nominal pore sizes of $\emptyset=1000 \mathrm{~nm}$. (B) Size distribution of proteoliposomes ( +syb 2) extruded through a polycarbonate membrane with nominal pore sizes of $\emptyset=400 \mathrm{~nm}$. This data was recorded by KUHLMANN.[90]

\section{Preparation of content labeled proteo-LUVs}

For the preparation of content labeled proteo-LUVs, the protocol was basically the same as for the preparation of membrane labeled proteo-LUVs. However, the lipid coupled fluorophore TxR-DPPE was displaced by POPE in the lipid films for proteo-SUV preparation. The unlabeled proteo-SUV suspension was transferred to a $5 \mathrm{~mL}$ round bottom flask and dried 
overnight at $4{ }^{\circ} \mathrm{C}$ to form MBSs. These proteo-lipid MBSs were then re-hydrated by incubation in $500 \mu \mathrm{L}$ content-label-buffer ( $25 \mathrm{~mm}$ HEPES, $50 \mathrm{mM} \mathrm{KCl,} 43 \mathrm{~mm}$ Sulforhodamine B, $1 \mathrm{~mm}$ DTT, $\mathrm{pH}$ 7.4) for at least $30 \mathrm{~min}$ at room temperature resulting in a suspension of multilamellar proteoliposomes. Extrusion (mini extruder, LiposoFast, Avestin) of this suspension through a polycarbonate membrane with a defined pore size yielded proteo-LUVs which were filled with the water soluble dye Sulforhodamine B. Subsequently, the proteo-LUV suspension was submitted to size exclusion chromatography (illustra NAP-10 G25 column, GE Healthcare, Little Chalfont, UK) in SNARE-buffer, to remove content-label-buffer which was not encapsulated, obtaining a suspension of content labeled proteo-LUVs in SNARE-buffer.

\section{Preparation of giant proteoliposomes (proteo-GUVs)}

The formation of giant unilamellar proteoliposomes (proteo-GUVs) started with the application of proteo-SUV suspension droplets $(2 \mu \mathrm{L})$ onto two indium tin oxide (ITO) covered glass slides, followed by drying in a desiccator over saturated sodium chloride solution overnight at $4{ }^{\circ} \mathrm{C}$. The slow drying process under high humidity conditions resulted in the formation of proteolipid MBSs on the ITO surfaces. The two glass slides were assembled into an electroformation chamber in which the proteo-lipid MBSs covered ITO surfaces faced each other in the middle of the chamber, as schematically shown in Figure 3-11.

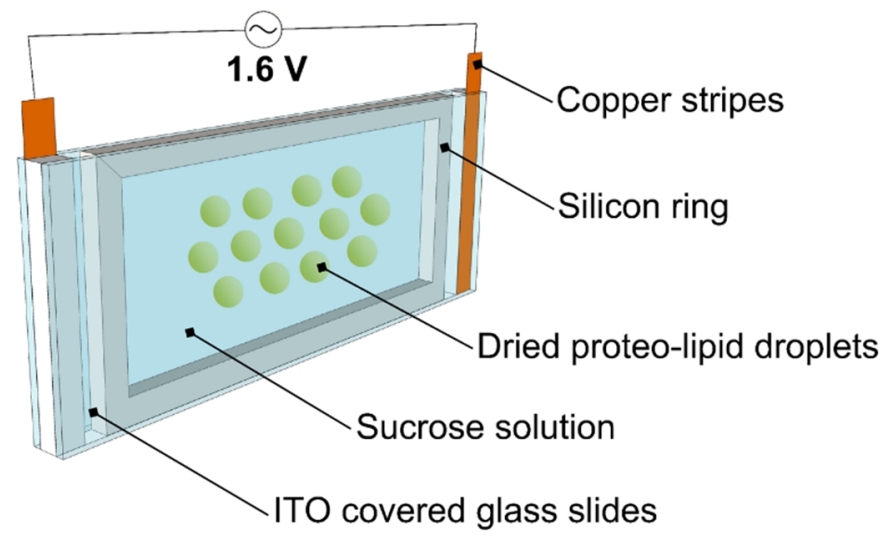

Figure 3-11 Schematic setup of an assembled electroformation chamber. A sinusoidal voltage of $1.6 \mathrm{~V}$ (peak to peak) with a frequency of $12 \mathrm{~Hz}$ was applied for electroformation on both copper electrodes for $3 \mathrm{~h}$.

A silicon ring between the two ITO slides served as sealing and allowed to fill the chamber with sucrose solution (200 mM). Via copper stripes, a sinusoidal voltage (1.6 V (peak to peak), $12 \mathrm{~Hz}$ ) was applied to the ITO surfaces for $3 \mathrm{~h}$ leading to proteo-GUV formation by swelling and pinching off from the proteo-lipid MBSs. The proteo-GUVs were harvested in fractions of $600 \mu \mathrm{L}$ by carefully pipetting from top. Each fraction was analyzed by widefield fluorescence microscopy to choose the fraction with the highest purity and amount of proteo-GUVs. 


\subsubsection{Preparation of pore-spanning membranes (PSMs)}

For the preparation of pore-spanning membranes (PSMs), porous substrates were rinsed with ethanol and dried under a stream of nitrogen gas. The substrates were cleaned in argon plasma (60 s, $p=0.015$ bar, $P=60 \%$ ) for further elimination of surface impurities using a Zepto plasma cleaner (Diener Electronic, Ebbhausen, Germany). Onto the cleaned substrates, a thin layer of titanium was applied by sputter coating (Cressington Sputter Coater 108auto, Watford, UK) for $25 \mathrm{~s}$ at $40 \mathrm{~mA}$ and 0.4 mbar, which served as crystallization nuclei for the subsequent coating with gold. That was done by thermal evaporation in high vacuum ( $p=5 \cdot 10^{-6}$ mbar) using a MED020 coating system (Bal-Tec, Leica, Wetzlar, Germany) to ensure an orthogonally applied gold layer with a thickness of 30-40 nm. Orthogonal coating exclusively on top of the porous substrate and not in the pore walls is of crucial importance for the subsequent formation of PSMs. The gold coated substrates were incubated in 6-mercapto-1-hexanol (6-MH, $c=1 \mathrm{mM}$ in $n$-propanol, overnight at $4{ }^{\circ} \mathrm{C}$ ) to form a densely packed self-assembled monolayer (SAM) on top of the substrate. For the last step of PSM preparation, the hydrophilically functionalized substrates were properly rinsed with ethanol and MilliQ and placed in a measuring chamber filled with SNARE buffer. GUV suspension (10-20 $\mu \mathrm{L})$ in isoosmolar sucrose solution was added above the porous part of the substrate, followed by incubation for at least $30 \mathrm{~min}$ at room temperature. PSMs were formed due to spontaneous rupture of GUVs adhered to the hydrophilic surface of the porous substrate. Residual lipid material as well as unspreaded GUVs were gently rinsed away by buffer exchange with a pipette. A schematic overview of all preparation steps is shown in Figure 3-12.

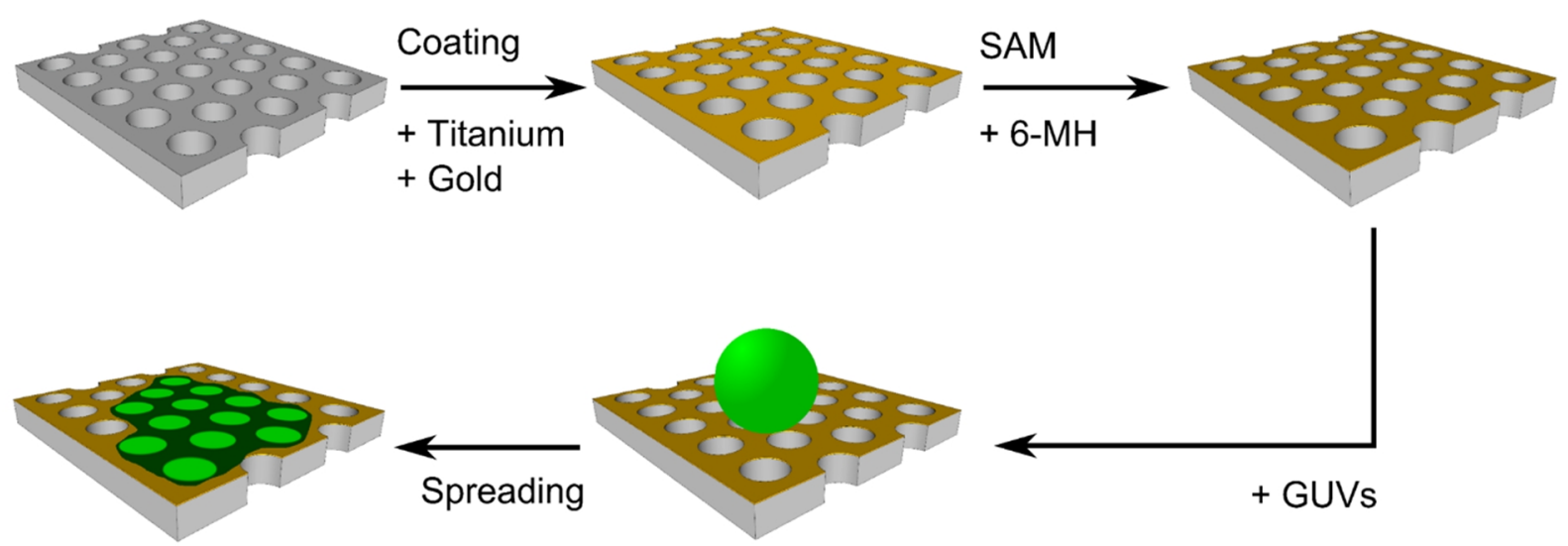

Figure 3-12 Schematic overview of the preparation steps to generate pore-spanning membranes (PSMs). Firstly, the substrates are coated with titanium and an orthogonal gold layer, followed by incubation in a 6-MH solution to form a self-assembled monolayer on top. Subsequent addition of GUVs to this hydrophilically functionalized surface leads to spreading and the formation of PSMs.[90] 


\subsubsection{Co-reconstitution of syb 2 and syt-1 into large proteoliposomes}

The so-called direct insertion method was applied for co-reconstitution of syb 2 and syt-1 into large unilamellar vesicles (LUVs). In this method, preformed LUVs were destabilized with the detergent $n$-OG, followed by addition and insertion of the particular proteins. To subsequently remove the detergent, size exclusion chromatography and dialysis were performed.

The co-reconstitution protocol started with the preparation of LUVs. For this purpose, a lipid film (0.5 mg, composition cf. Table 3-12) was allowed to swell in SNARE buffer (500 $\mu \mathrm{L}$, composition cf. Chapter 3.2.3) for $30 \mathrm{~min}$ at room temperature. During this incubation period, the mixture was vortexed three times for $30 \mathrm{~s}$ in a 5 min time interval, yielding an opaque suspension of multilamellar vesicles. From this suspension, LUVs were obtained by extrusion (LiposoFast-Basic, Avestin, Ottawa, Canada) through a polycarbonate membrane with a nominal pore size of $400 \mathrm{~nm}(31 \mathrm{x})$. The resulting LUVs were destabilized by addition of $n$-OG $(c(n-\mathrm{OG})=26 \mathrm{mM})$, followed by incubation for $10 \mathrm{~min}$ at room temperature. Subsequently, protein solutions of syb 2 and syt-1 were added, adjusting a protein/lipid-ratio of 1:500 and 1:1000, respectively. This mixture was further incubated at room temperature for $30 \mathrm{~min}$, followed by size exclusion chromatography in MilliQ/SNARE buffer (9:1) using a G-25 prepacked column (illustra NAP-25 G-25, GE Healthcare, Little Chalfont, UK). After size exclusion, the proteo-LUVs containing fraction $(850 \mu \mathrm{L})$ was submitted to concentration (Concentrator 5301, Eppendorf, Hamburg, Germany) resulting in a final volume of $\sim 400 \mu \mathrm{L}$. The suspension was transferred to a dialysis cassette (Slide-A-Lyzer, $0.1-0.5 \mathrm{~mL}$, $M W C O=3.5 \mathrm{kDa}$, Thermo Fischer Scientific, Waltham, MA, USA) and dialyzed against SNARE-buffer $(500 \mathrm{~mL})$ supplemented with BioBeads $(1 \mathrm{~g})$ overnight at $4{ }^{\circ} \mathrm{C}$. To enhance detergent removal, a second size exclusion step (illustra NAP-10 G-25 column, GE Healthcare, Little Chalfont, UK), this time in SNARE buffer, was performed, yielding the proteo-LUVs used for fusion experiments.

\subsubsection{Isolation and purification of chromaffin granules}

To analyze chromaffin granule (CG) fusion in vitro and on a single vesicle level, CGs had to be isolated and purified from bovine adrenal medulla. The isolation was performed in the lab of REINHARD JAHN (Max-Plack-Institut für biophysikalische Chemie, Göttingen) with the kind help of YONGSOO PARK. The whole isolation was performed at $4{ }^{\circ} \mathrm{C}$ and started with dissecting a bovine adrenal gland $(810 \mathrm{~g})$ to gain pure adrenal medulla tissue. After mincing the obtained tissue with a scissor in $50 \mathrm{~mL} 0.3 \mathrm{M}$ sucrose buffer (10 mM HEPES, $0.3 \mathrm{M}$ sucrose, $200 \mu \mathrm{M}$ 
PMSF, pH 7.4), the debris was homogenized in a Glass/Teflon homogenizer (IKA Labortechnik; Germany) at $1200 \mathrm{rpm}$ (7-8 strokes). The homogenate was centrifuged (1000 x g, $15 \mathrm{~min}, 4^{\circ} \mathrm{C}$, rotor: SS-34), the pellet discarded and the supernatant was centrifuged again $\left(12000 \mathrm{xg}, 15 \mathrm{~min}, 4{ }^{\circ} \mathrm{C}\right.$, rotor: SS-34). After the second step of centrifugation, the supernatant was discarded and the pellet resuspended in $35 \mathrm{~mL} 0.3 \mathrm{M}$ sucrose buffer. This procedure was repeated twice, the pellets were pooled and resuspended in a maximum volume of $0.5 \mathrm{~mL} 0.3 \mathrm{M}$ sucrose buffer. After this differential centrifugation, the crude granule fraction (CGF) was separated from contaminating organelles on a continuous sucrose density gradient (from $0.3 \mathrm{M}$ to $2 \mathrm{M}$ ). The gradient was prepared by transferring $5 \mathrm{~mL} 2 \mathrm{M}$ sucrose buffer $(10 \mathrm{mM}$ HEPES, $2 \mathrm{M}$ sucrose, $\mathrm{pH} 7.4$ ) to a centrifuge tube and carefully adding $5 \mathrm{~mL} 0.3 \mathrm{M}$ sucrose buffer on top. A linear and continuous gradient was achieved with the Gradient Master (BioComp Instruments, Canada) on top of which the CGF was loaded. Due to their high protein content, CGs exhibit a buoyant density that is higher than most of the other organelles, with the result that a subsequent ultracentrifugation $\left(110000 \mathrm{xg}, 1 \mathrm{~h}, 4{ }^{\circ} \mathrm{C}\right.$, rotor: $\left.\mathrm{SW} 41 \mathrm{Ti}\right)$ yielded mature CGs in the bottom fraction of the gradient. Subsequent to ultracentrifugation, the supernatant was discarded, the pellet (pure chromaffin granules, CGs) was gently washed twice with $1 \mathrm{~mL}$ k-glutamate buffer $\left(120 \mathrm{mM} \mathrm{KC}_{5} \mathrm{H}_{8} \mathrm{NO}_{4}, 20 \mathrm{mM} \mathrm{KCH}_{3} \mathrm{COO}, 20 \mathrm{mM}\right.$ HEPES, $\mathrm{pH}$ 7.4) and the CGs were resuspended in $250 \mu \mathrm{L} \mathrm{k-glutamate}$ buffer. Prior to their storage at $-80{ }^{\circ} \mathrm{C}$, the resuspended CGs were divided in aliquots of $4 \mu \mathrm{L}$. The high purity of mature CGs using this isolation protocol was confirmed by PARK, performing western blots, which showed the depletion of markers of other organelles in the bottom fraction. Beyond that, a heterogeneous size distribution of purified CGs with an average diameter of $167.7 \pm 14.3 \mathrm{~nm}$ was determined using cryo-electron microscopy. ${ }^{[103]}$

\subsubsection{Fluorescent labeling of chromaffin granules}

To detect single vesicle fusion of chromaffin granules (CGs) with pore-spanning membranes (PSMs) by means of fluorescence microscopy, the CGs had to be marked with a fluorescent probe. For this purpose the far-red fluorescent and lipophilic dye DiD-C 18 (Table 3-2) was chosen.

The labeling protocol started with the dilution of a $\mathrm{CG}$ aliquot $(4 \mu \mathrm{L})$ in SNARE buffer $(150 \mu \mathrm{L})$, followed by the addition of DiD-C ${ }_{18}$ labeling solution $(4.05 \mu \mathrm{L}$, Table $3-13)$ to adjust a final concentration of $c\left(\mathrm{DiD}-\mathrm{C}_{18}\right)=2.5 \mu \mathrm{M}$. 
Table 3-13 Composition of the DiD-C18 labeling solution.

\section{Volume fraction}

\begin{tabular}{lll}
\hline DiD-C18-stock & DiD-C $_{18}(1 \mathrm{mM}$ in DMSO $)$ & 1 \\
n-OG-solution & $n$-OG (50 mM in SNARE buffer $)$ & 9 \\
\hline
\end{tabular}

The resulting mixture was incubated for $1 \mathrm{~h}$ at room temperature, transferred to a dialysis cassette (Slide-A-Lyzer, $0.1-0.5 \mathrm{~mL}, M W C O=3.5 \mathrm{kDa}$ ) and dialyzed against SNARE buffer $(500 \mathrm{~mL})$ supplemented with Bio-Beads $(1 \mathrm{~g})$ for $2.5 \mathrm{~h}$ at $4{ }^{\circ} \mathrm{C}$.

\subsubsection{Density gradient centrifugation}

Density gradient centrifugation (DGC) is a procedure to separate molecules or cell components through a medium of graded density. For this purpose, the sample is placed on top of a gradient and exposed to centrifugal force. The molecules or cell components are separated and 'banded' in the gradient based on their buoyant density and can be collected as pure fractions.

DGC was used to qualitatively analyze the reconstitution efficiency of transmembrane proteins into vesicles. To this end, proteoliposome suspension $(40 \mu \mathrm{L})$ was mixed with $80 \%$-Nycodenz solution $(40 \mu \mathrm{L}, 80 \%(w / v))$ and transferred to an ultracentrifuge tube. The resulting mixture was gradually overlayed with $30 \%$-Nycodenz solution $(40 \mu \mathrm{L}, 30 \%(w / v))$ and SNARE buffer $\left(20 \mu \mathrm{L}\right.$ ). Ultracentrifugation for $2.5 \mathrm{~h}$ at $45000 \mathrm{rpm}$ and $4{ }^{\circ} \mathrm{C}$ (Centricon T-1065, rotor: TST 60.4, Kontron Instruments, Osterode am Harz, Germany) led to the formation of a continuous Nycodenz gradient from $0-40 \%(w / v)$, in which the buffer filled proteoliposomes on top of the gradient were segregated from unreconstituted protein in the bottom fractions (Figure 3-13). After centrifugation, 7 fractions of $20 \mu \mathrm{L}$ were gradually collected from top and further analyzed by SDS-PAGE with regard to their protein content. 


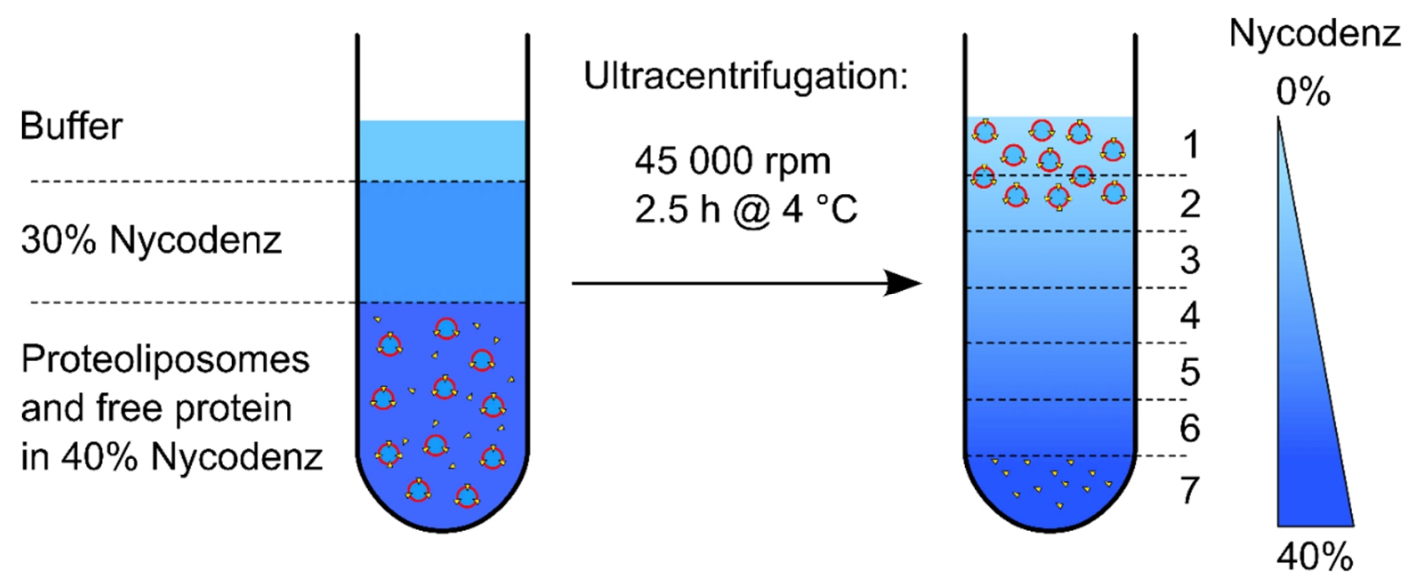

Figure 3-13 Schematic illustration of the procedure of density gradient centrifugation (Nycodenz assay) to segregate proteoliposomes (red circles) from unreconstituted, free protein (yellow triangle) by ultracentrifugation in a Nycodenz gradient (0-40 \% (w/v)).

\subsubsection{Phosphate determination assay}

The protocol for the preparation of proteoliposomes, independent of their size (LUVs or SUVs), resulted in slight differences for each individual preparation regarding the proteoliposome concentration of the obtained suspension. To verify comparability, especially in bulk fusion experiments of two different liposome populations, the lipid concentration was determined prior to the fusion experiment. This was done indirectly by analyzing the phosphate content of a defined volume of the proteoliposome suspension utilizing an assay based on the reaction of in situ generated phosphomolybdate with malachite green to form a colored complex under acidic conditions. ${ }^{[104]}[105]$

The protocol started with combining solutions of malachite green $(0.2 \%(w / v)$ in MilliQ) and ammonium molybdate tetrahydrate $\left(\left(\mathrm{NH}_{4}\right)_{6} \mathrm{Mo}_{7} \mathrm{O}_{24} \cdot 4 \mathrm{H}_{2} \mathrm{O}, 10 \%(w / v)\right.$ in $\left.4 \mathrm{M} \mathrm{HCl}\right)$ in a ratio of 3:1, followed by centrifugation for $5 \mathrm{~min}$ at $3800 \mathrm{rpm}$ (Allegra TM X-22R, rotor: SX4250, Beckman Coulter, Brea, CA, USA). The resulting supernatant was referred to as phosphatereagent. For the development of a calibration curve, a dilution series of phosphate standards (Table 3-14) was prepared.

Table 3-14 Dilution series of phosphate standards used for the development of a calibration curve.

\begin{tabular}{lcccccccccc}
\hline Phosphate standard & $\mathbf{0}$ & $\mathbf{1}$ & $\mathbf{2}$ & $\mathbf{3}$ & $\mathbf{4}$ & $\mathbf{5}$ & $\mathbf{6}$ & $\mathbf{7}$ & $\mathbf{8}$ & $\mathbf{9}$ \\
\hline $\mathbf{H C l}(\mathbf{1 . 2} \mathbf{M}) / \mathbf{\mu L}$ & 600 & 595 & 590 & 585 & 580 & 575 & 570 & 560 & 550 & 540 \\
$\mathbf{K H}_{2} \mathbf{P O}_{4}(\mathbf{1 0 0} \mathbf{~ \mu M}) / \mathbf{\mu L}$ & 0 & 5 & 10 & 15 & 20 & 25 & 30 & 40 & 50 & 60 \\
$\mathbf{n}\left(\mathbf{P O}_{4}{ }^{3-}\right) / \mathbf{n m o l}$ & 0.0 & 0.5 & 1.0 & 1.5 & 2.0 & 2.5 & 3.0 & 4.0 & 5.0 & 6.0 \\
\hline
\end{tabular}


To determine the phosphate content of a proteoliposome suspension, samples of 20-50 $\mu \mathrm{L}$ were taken and submitted to incineration by adding $200 \mu \mathrm{L}$ of perchloric acid $(70 \%(v / v))$ and incubating for $\sim 1 \mathrm{~h}$ at $220{ }^{\circ} \mathrm{C}$. Subsequently, the remaining solid was dissolved in $600 \mu \mathrm{L} \mathrm{HCl}$ $(1.2 \mathrm{M})$ and $200 \mu \mathrm{L}$ of the phosphate-reagent was added to the samples as well as to each of the ten phosphate standards. After incubation at room temperature for $10 \mathrm{~min}$, the absorption at $\lambda=660 \mathrm{~nm}\left(A_{660}\right)$ was measured. This procedure was carried out three times for each standard and each sample, whereas the phosphate standard 0 (Table 3-14) was taken as a blank. The measured $A_{660}$ values of the standards were plotted against their phosphate content yielding a calibration curve, to which a linear regression was fitted. Insertion of the averaged $A_{660}$ values of the samples taken from proteoliposome suspensions into the linear Equation provided the amount of phosphate in these samples.

\subsection{Biophysical methods}

In the frame of this work, microscopic as well as spectroscopic methods based on the principal of fluorescence were applied to analyze in vitro SNARE-mediated membrane fusion.

\subsubsection{Theoretical basics of fluorescence}

The process of spontaneous photon emission of a molecule that was electronically excited beforehand is called fluorescence. During this process, the molecule (fluorophore) returns to its electronical ground state. Fluorescence exclusively occurs if the fluorophore is excited via electromagnetical radiation and decays within nanoseconds. ${ }^{[106]}$ The excitation is induced by the absorption of light with a fluorophore specific wavelength, leading to the transition of an electron from the electronic ground state $\left(S_{0}\right)$ to an electronically excited singlet state $\left(S_{1}\right)$. According to Franck-Condon's principle, a rule in spectroscopy based on quantum mechanics, the transition of an electron occurs most likely to a vibronically excited state vertical to the vibrational ground state $\left(v^{\prime \prime}=0\right)$ of $S_{0}$. This is caused by the much faster transition times of electrons $\left(\sim 10^{-15} \mathrm{~s}\right)$ in comparison to nuclei displacement $\left(\sim 10^{-13} \mathrm{~s}\right)$. Beyond that, the quantum mechanical consideration of this principle states that the intensity of a vibronic transition is proportional to the square of the overlap integral between the vibrational wavefunctions of the two states that are involved in the transition. ${ }^{[107]}$ Once in a vibrationally excited state of $S_{1}$, fluorophores quickly relax non-radiatively to the lowest vibrational level $\left(v^{\prime}=0\right)$ and, from 
there, return to the electronic ground state $\left(S_{0}\right)$ via photon emission. The process of fluorescence resembling the requirements quoted above is schematically depicted in Figure 3-14.

A

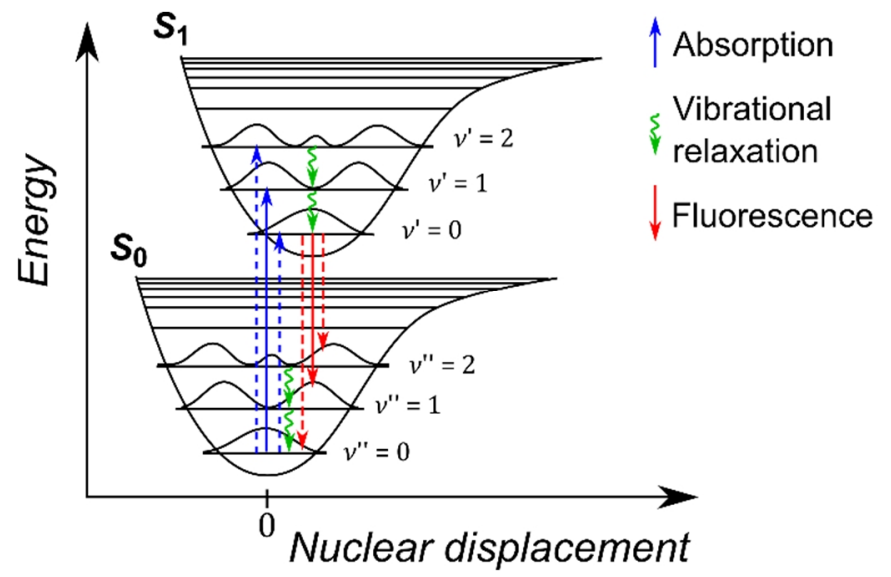

B

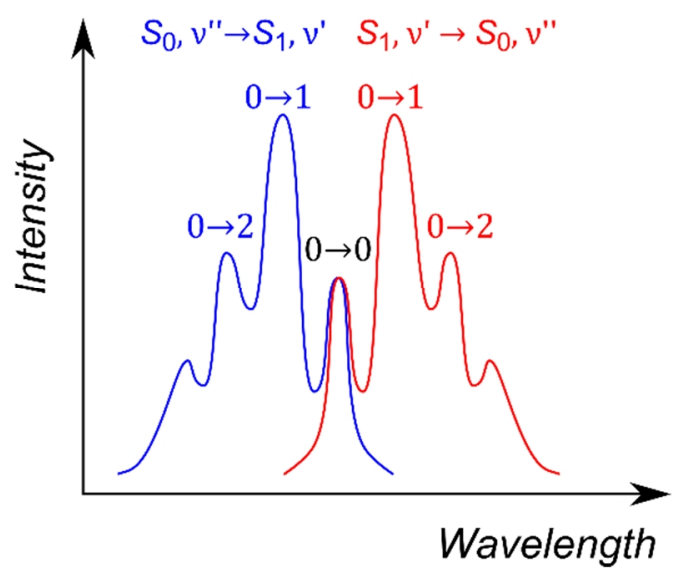

Figure 3-14 Schematic display of the process of fluorescence following Franck-Condon's principle modified according to SCHWAMBORN. ${ }^{[108]}$ (A) 2D potential curves for the electronic ground state ( $\mathrm{S}_{0}$ ) and the electronically excited state $\left(\mathrm{S}_{1}\right)$ of a fluorophore. The horizontal lines stand for the vibrational states $\mathrm{v}^{\prime \prime}$ of $\mathrm{S}_{0}$ and $\mathrm{v}^{\prime}$ of $\mathrm{S}_{1}$. Vibronic transitions due to light absorption $\left(\mathrm{S}_{0} \rightarrow \mathrm{S}_{1}\right)$ are depicted as blue arrows, whereas those for relaxation in terms of fluorescence are shown in red. Non-radiative vibrational relaxation within the individual electronic state $S_{0}$ or $S_{1}$ are depicted as green arrows. (B) Schematic spectrum for absorption and emission reflecting the probability for specific vibronic transitions between $\mathrm{S}_{0}$ and $\mathrm{S}_{1}$. ${ }^{[109]}$ [110]

In most cases, the photons emitted during relaxation exhibit a lower energy in comparison to those absorbed for excitation. The encountered energy difference between absorption and emission is called Stokes shift, named after the Irish physicist GEORGE G. STOKES. It is the result of mainly two actions: non-radiative vibrational relaxation due to collisions or dissipation due to solvent reorganization. The second action is attributed to the fact that a fluorophore is a dipole, surrounded by a solvent. After entering an electronically excited state, its dipole moment will change, causing a delayed and energy consuming rearrangement of the solvent molecules in direct surrounding. Figure 3-15 shows the absorption and emission spectrum for the fluorophore HisZiFiT with a Stokes shift of $35 \mathrm{~nm}$.

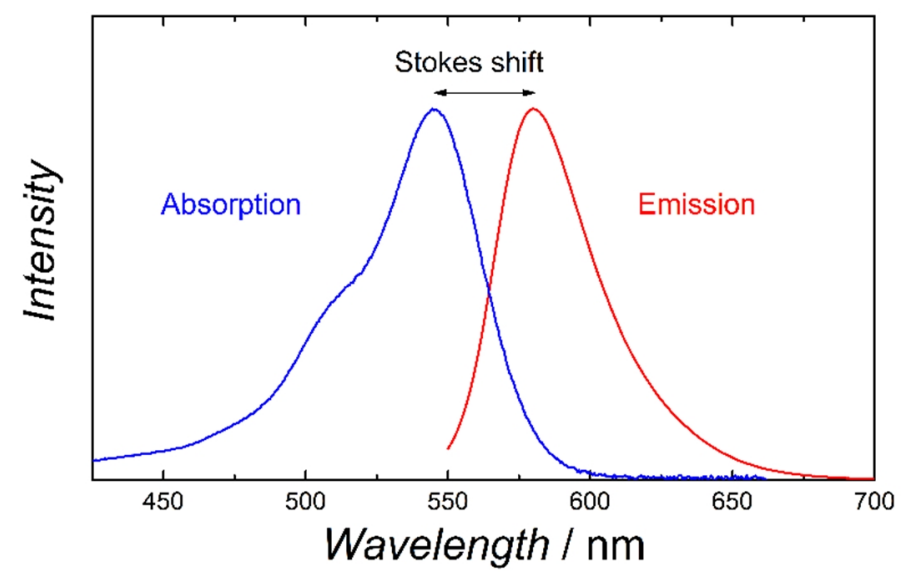

Figure 3-15 Absorption and emission spectrum of the fluorescent dye HisZiFiT with a Stokes shift of $35 \mathrm{~nm}$. 


\subsubsection{Förster resonance energy transfer (FRET)}

For excited atoms or molecules, a multitude of relaxation processes are known. Aside from radiative deactivation in terms of fluorescence or phosphorescence, also non-radiative processes, like vibrational relaxation or internal conversion in combination with vibrational relaxation can transition excited molecules to the ground state $S_{0}$. Another non-radiative relaxation pathway of an excited fluorophore is based on dipole-dipole coupling with a second fluorophore in its ground state. Here, the excited donor-fluorophore relaxes to its ground state via resonance energy transfer to an acceptor-fluorophore. This leads to excitation of the acceptor-fluorophore, which, in turn, can undergo deactivation through vibrational relaxation followed by fluorescence. This mechanism, resulting in an energy transfer between two distinct fluorophores (donor and acceptor), was named Förster resonance energy transfer (FRET) after its discoverer, THEODOR FÖRSTER. The fact that FRET efficiency ( $\left.E_{\mathrm{FRET}}\right)$ was determined to be reciprocal proportional to the sixth power of the distance between donor and acceptor $(R)$, makes FRET an extremely sensitive tool to analyze small changes in fluorophore distances. This dependence is expressed in Equation (3-1).

$$
E_{\mathrm{FRET}}=\frac{1}{1+\left(\frac{R}{R_{0}}\right)^{6}}
$$

In this Equation (3-1), $R_{0}$ stands for the Förster radius, a constant which has to be determined experimentally and is specific for every pair of donor and acceptor $\left(E_{\mathrm{FRET}}\left(R_{0}\right)=50 \%\right)$. A sequence of all steps, which are passed during FRET between two fluorophores, is schematically illustrated in a Jablonski diagram in Figure 3-16 as well as an exemplary plot of $E_{\mathrm{FRET}}$ as a function of the fluorophore distance.

A

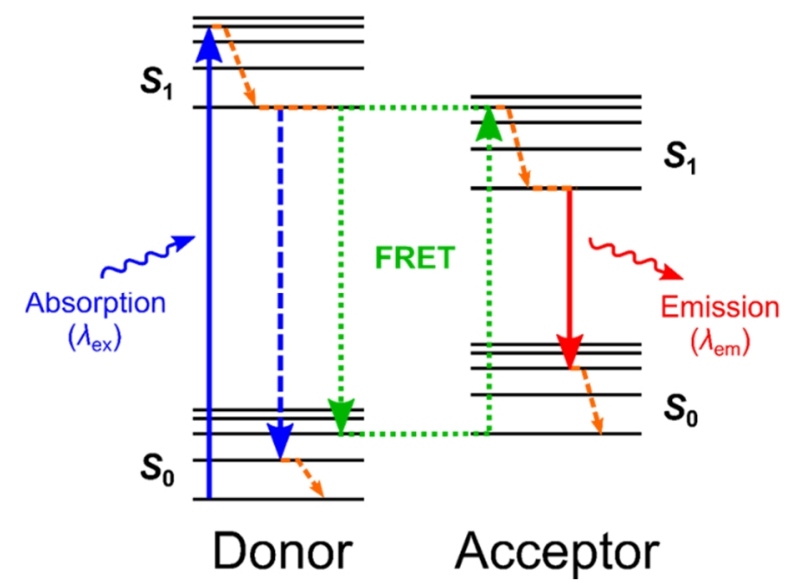

B

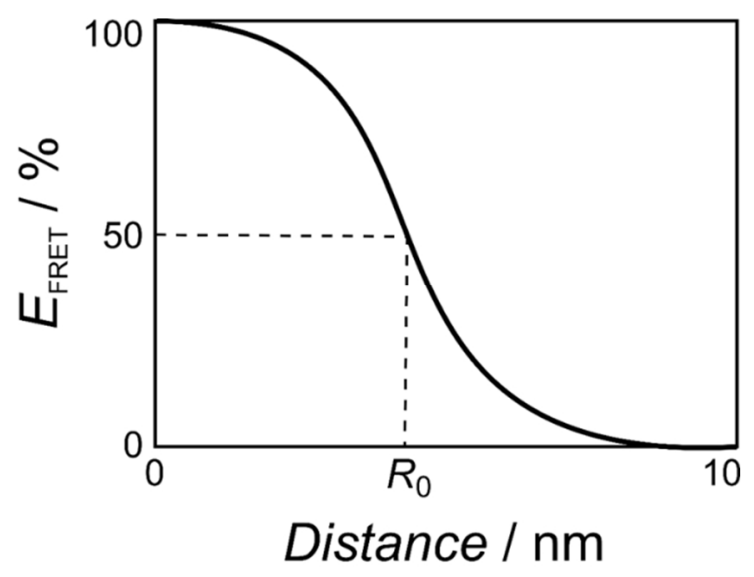

Figure 3-16 (A) Jablonski diagram showing all steps occurring during FRET between a donor- and an acceptor-fluorophore. (B) Exemplary plot of $\mathrm{E}_{\mathrm{FRET}}$ as a function of fluorophore distance. 
From Figure 3-16B and Equation (3-1) it becomes obvious that one main criterion for FRET between a donor- and an acceptor-fluorophore is spatial proximity ( 10-80 ̊). Furthermore, the energy transferred by the donor has to fit the energy which is needed to excite the acceptor. In order to fulfil that, the emission spectrum of the donor has to significantly overlap with the absorption spectrum of the acceptor.

In this work, the FRET-pair Atto488-DPPE and TxR-DPPE was used to analyze lipid mixing of two distinct artificial membrane systems. The absorption and emission spectra of both fluorophores are depicted in Figure 3-17.

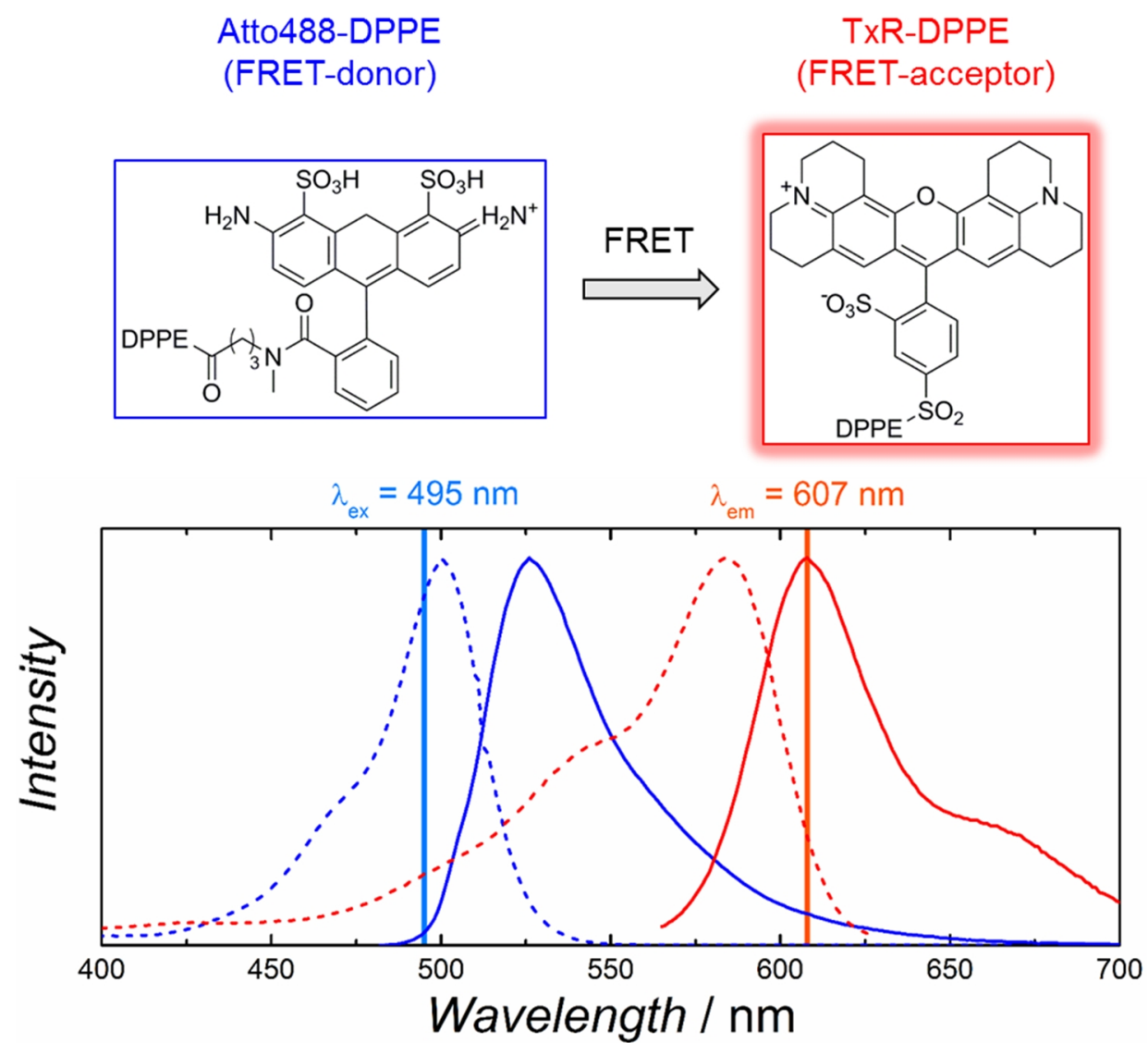

Figure 3-17 Schematic illustration of the FRET-effect between the two lipid-coupled fluorophores Atto488DPPE (FRET-donor) and TxR-DPPE (FRET-acceptor). The two fluorophores exhibit a significant overlap between the emission spectrum of the donor (solid curve, blue) and the absorption spectrum of the acceptor (dashed curve, red) which is a prerequisite for FRET. By exciting the donor at $\lambda_{\mathrm{ex}}=495 \mathrm{~nm}$, emission of the acceptor can be detected at $\lambda_{\mathrm{em}}=607 \mathrm{~nm}$ if both fluorophores are in close spatial proximity. 


\subsubsection{Fluorescence spectroscopy}

Fluorescence spectroscopy is a form of electron spectroscopy in which the samples contain fluorescent probes (fluorophores). By exciting these fluorophores with light of a specific wavelength and taking advantage of the Stokes shift, it is possible to detect the red-shifted fluorescence exclusively. For this purpose, to isolate the incident light and the fluorescent light, diffraction grating monochromators or filters are used. A simplistic design of the setup of a fluorescence spectrometer is shown in Figure 3-18.

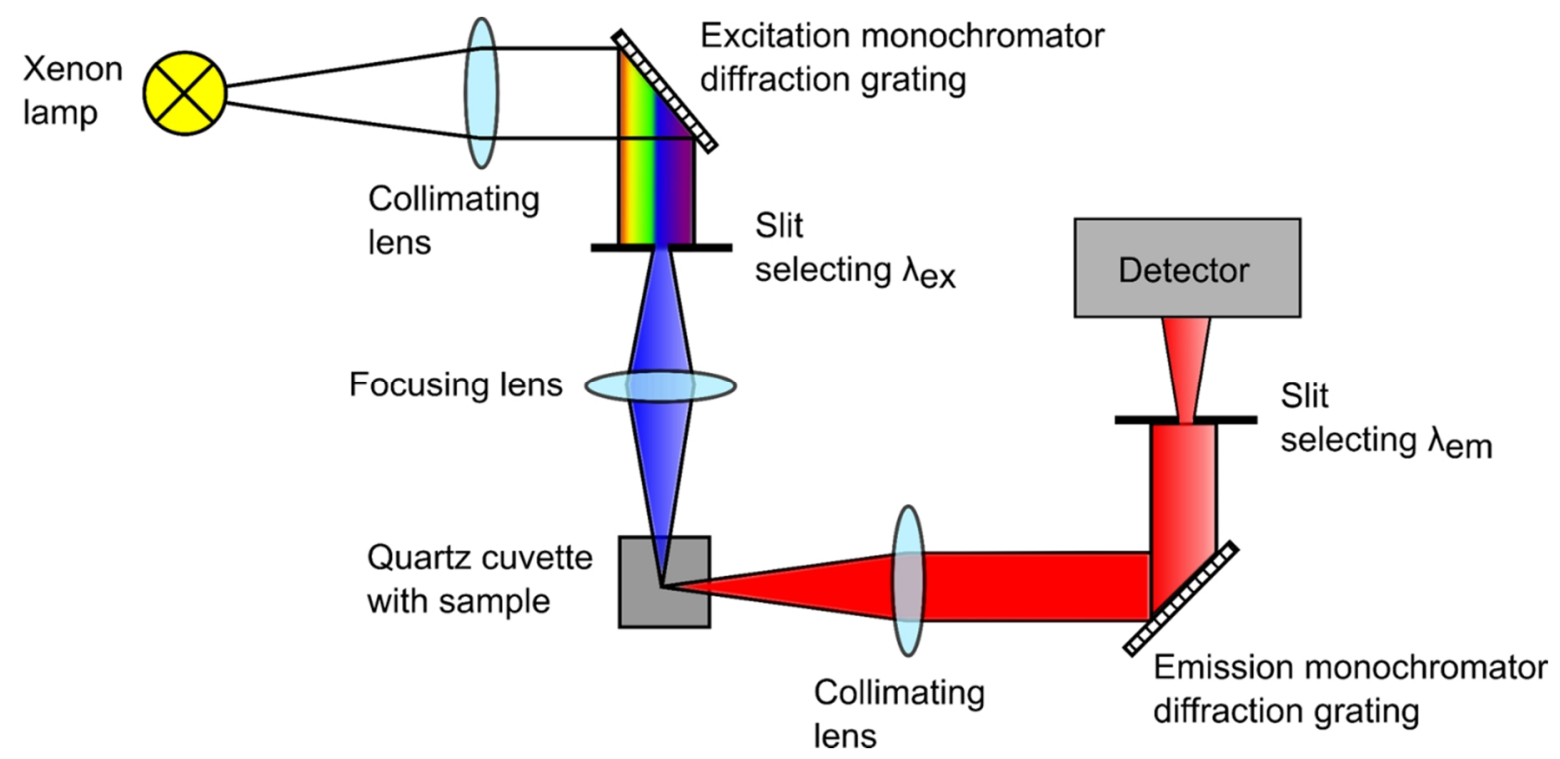

Figure 3-18 Schematic display of the setup of a fluorescence spectrometer modified according to SCHWAMBORN. ${ }^{[108]}$ Light, with the desired wavelength for excitation $\left(\lambda_{\text {ex }}\right)$, can be obtained by a diffraction grating in combination with an aperture with a defined slit width (Excitation monochromator). The emitted fluorescence is collected orthogonally to the excitation beam to minimize the detection of excitation light. It is focused by a collimating lens on an emission monochromator, with which the emission wavelength of interest can be selected for detection.

Fluorescence spectroscopy was performed to analyze SNARE-mediated vesicle-vesicle bulk fusion, utilizing the FRET-effect between Atto488-DPPE and TxR-DPPE as an indicator for lipid mixing. For this purpose, two populations of vesicles were prepared, one containing $1 \mathrm{~mol} \%$ Atto488-DPPE and the other $1 \mathrm{~mol} \%$ TxR-DPPE. To induce membrane fusion, $\Delta \mathrm{N} 49$ was reconstituted into the Atto488-DPPE doped population and syb 2 was integrated into the TxR-DPPE labeled population. Both proteins were reconstituted in a protein/lipid ratio of 1:500. Due to the fact that FRET is extremely sensitive to the distance between donor(Atto488-DPPE) and acceptor-fluorophore (TxR-DPPE), the resonance energy transfer was only observable upon lipid mixing between the two distinct vesicle populations in case of successful SNARE-mediated fusion. SNARE specificity was proven by blocking the $\Delta$ N49 containing vesicle population with an excess of syb 2 (1-96) prior to the fusion experiment. 


\section{Experimental procedure}

SNARE-mediated vesicle-vesicle bulk fusion experiments were all carried out using the fluorescence spectrometer FP-6500 (JASCO, Pfungstadt, Germany). An overview of the sequence of steps performed during bulk fusion experiments is shown in Figure 3-19.

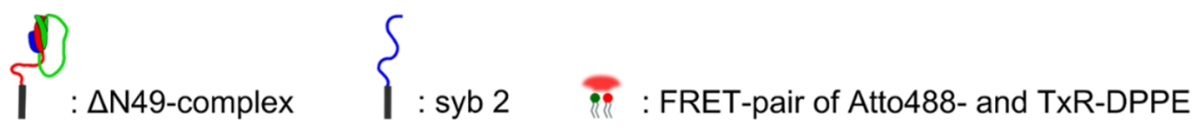

1. Addition of vesicle population 1 ( $\triangle N 49$; Atto488-DPPE) in SNARE buffer to a quartz cuvette:

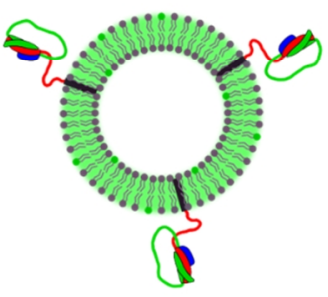

Population 1 record of the emission spectrum of vesicle popultaion 1

Excitation: $495 \mathrm{~nm}$

Detection: $500-700 \mathrm{~nm}$

2. Start of the time dependent record of FRET-acceptor fluorescence and addition of vesicle population 2 (syb 2; TxR-DPPE):

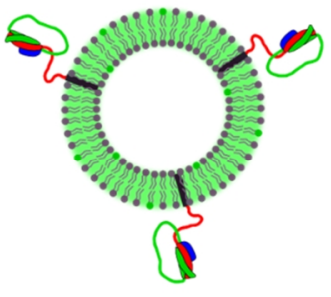

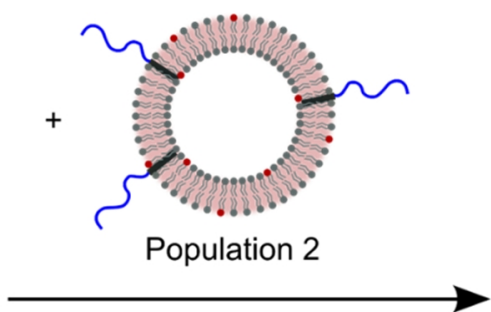

Excitation: $495 \mathrm{~nm}$

Detection: $607 \mathrm{~nm}$ vs. time
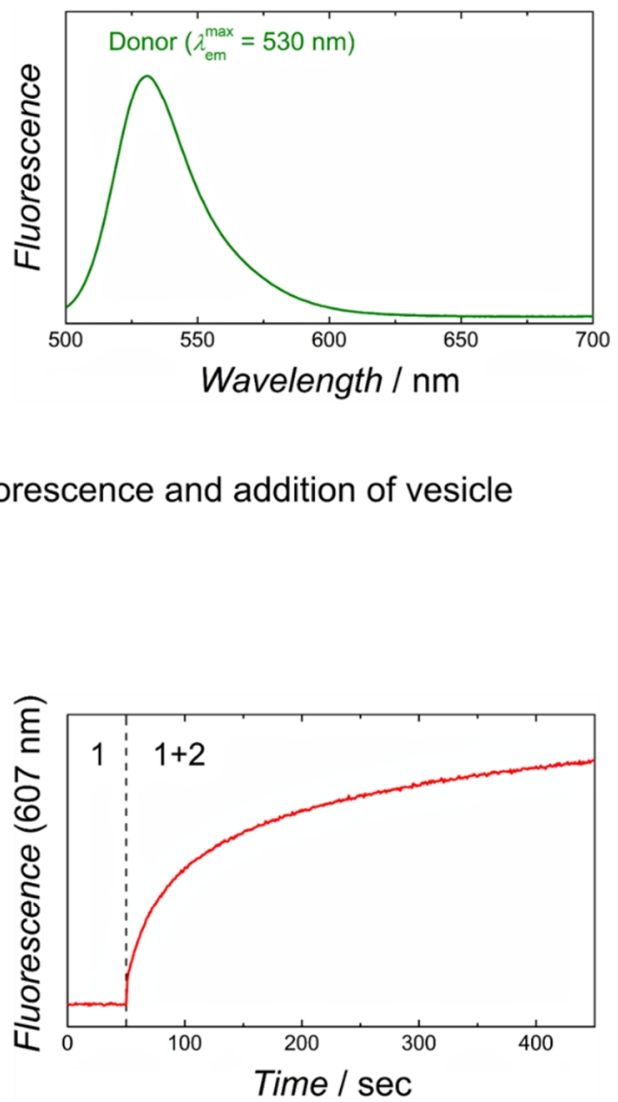

3. Record of the emission spectrum after the fusion experiment:

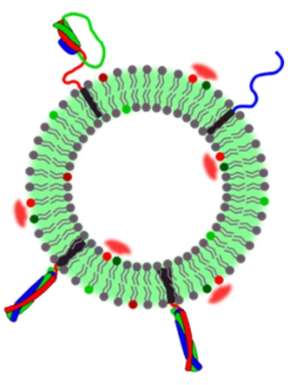

Population 1+2 record of the emission spectrum of vesicle popultaion $1+2$ after fusion

Excitation: $495 \mathrm{~nm}$

Detection: $500-700 \mathrm{~nm}$

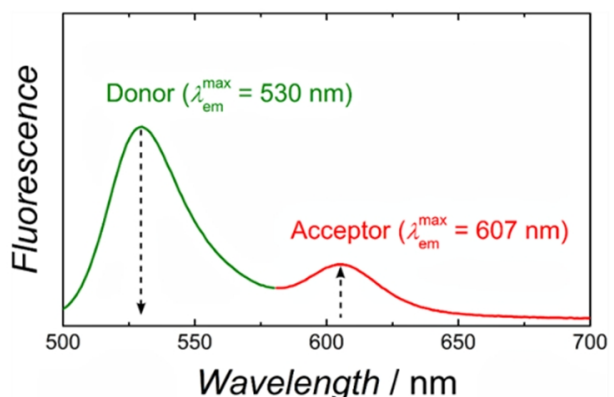

Figure 3-19 Sequence of steps carried out during SNARE-mediated vesicle-vesicle bulk fusion experiments. 
The protocol started with the addition of vesicle population $1(75 \mu \mathrm{L}, \Delta \mathrm{N} 49$, Atto488-DPPE) in SNARE buffer $(550 \mu \mathrm{L})$ to a quartz cuvette, followed by the record of an emission spectrum of the FRET-donor in absence of the FRET-acceptor $\left(\lambda_{\mathrm{ex}}=495 \mathrm{~nm}\right.$, detection range: 500 $700 \mathrm{~nm})$. Subsequently, a time dependent measurement of the fluorescence intensity at $\lambda=607 \mathrm{~nm}(F(607 \mathrm{~nm}))$ was started, which corresponds to the maximum emission wavelength of the FRET-acceptor. This measurement was performed with an excitation wavelength still at $\lambda_{\mathrm{ex}}=495 \mathrm{~nm}$. Vesicle population $2(75 \mu \mathrm{L}$, syb 2, TxR-DPPE) was added, followed by further recording of $F(607 \mathrm{~nm})$ for $\sim 30 \mathrm{~min}$. After finishing the time dependent measurement of $F(607 \mathrm{~nm})$, an emission spectrum of this mixture of population 1 and 2 after fusion was recorded $\left(\lambda_{\mathrm{ex}}=495 \mathrm{~nm}\right.$, detection range: $500-700 \mathrm{~nm}$ ). All vesicle-vesicle bulk fusion experiments were carried out at $37^{\circ} \mathrm{C}$, if not stated differently. The volumes given above were used standardly to control the reconstitution of active SNAREs into liposomes. For quantitative analysis of vesicle-vesicle bulk fusion under different conditions (e.g. lipid mixtures $\left( \pm \mathrm{PI}(4,5) \mathrm{P}_{2}\right)$, proteins $\left( \pm\right.$ syt-1) or salts $\left.\left( \pm \mathrm{Ca}^{2+}\right)\right)$, the proteoliposome concentration of both populations was indirectly determined by phosphate assay. To assure comparable conditions, the applied volumes of vesicle suspensions were calculated to result in a phosphate content of $n\left(\mathrm{PO}_{4}{ }^{3-}\right)=10 \mathrm{nmol}$ and the volume of the SNARE buffer was always adjusted to result in a total volume of $700 \mu \mathrm{L}$ in the quartz cuvette. For control experiments, vesicle population 1 (with reconstituted $\Delta \mathrm{N} 49$ ) was incubated with a protein solution of syb 2 (1-96) prior to the fusion experiment.

\subsubsection{Confocal laser scanning microscopy (CLSM)}

For the determination of SNARE-mediated single vesicle fusion, the method of confocal laser scanning microscopy (CLSM) was applied. In CLSM, the sample is scanned with a collimated laser beam and only fluorescence of a spot in a defined focal plane is detected. This is achieved by galvanometer mirrors modifying the light path to allow for spot-by-spot scanning of the sample in $\mathrm{x}-\mathrm{y}$ plane and a detector pinhole, which blocks fluorescent stray light not originating from the focal plane. The advantage of this method is, that only a small focal volume of the sample is illuminated, which minimizes photo damage and fluorophore bleaching. However, this principle results in a limited image acquisition rate by the scanning velocity. An illustration of the general setup and light path of a confocal microscope is shown in Figure 3-20. 


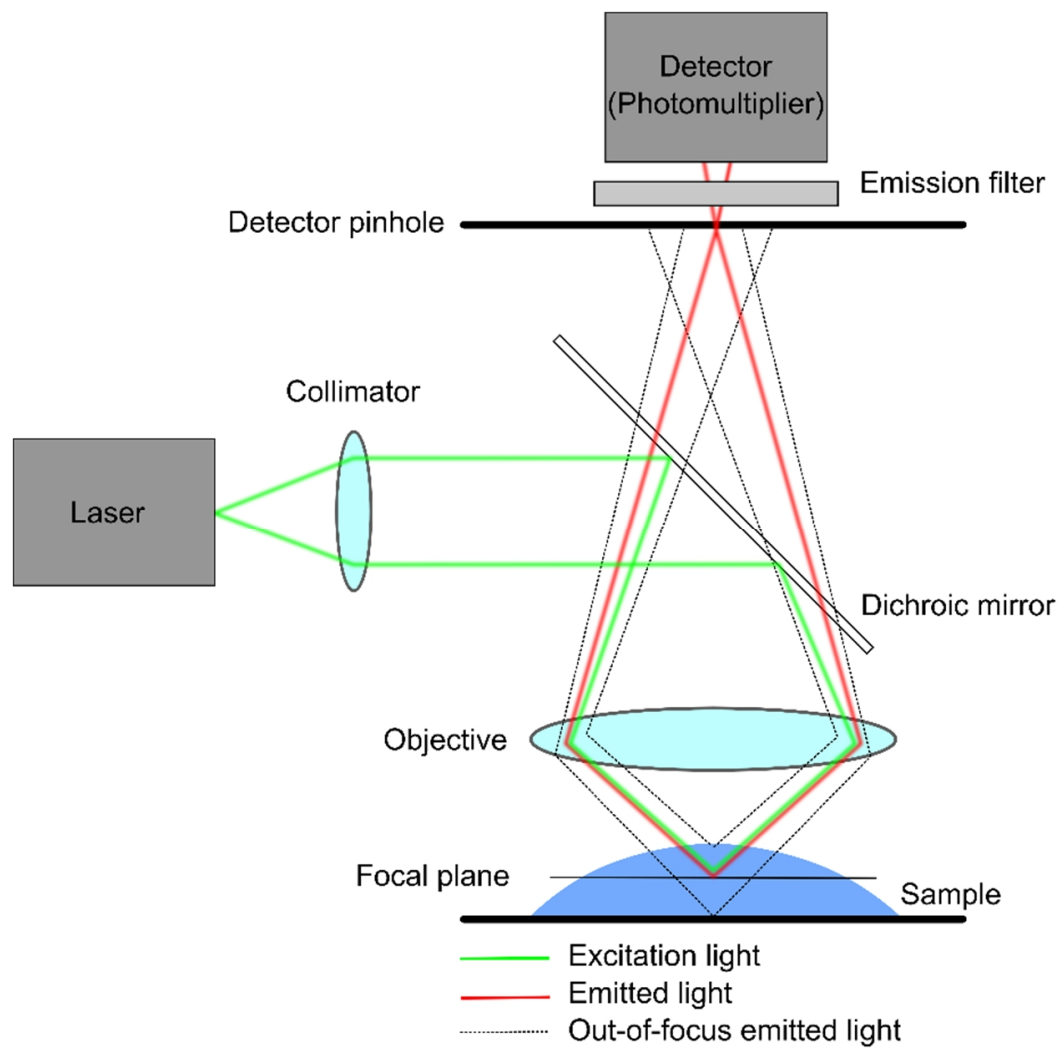

Figure 3-20 Schematic drawing of the general setup and light path of a confocal microscope, modified according to SCHÜTTE.[111]

The central element of a confocal microscope is the adjustable detector pinhole, which protects the detector, a photomultiplier, from out-of-focus emitted light (dashed lines Figure 3-20). The lateral resolution achieved in confocal microscopy is limited by Abbe's law. By applying this law, the resolution $\left(d_{\text {FWHM }}\right)$ to separate two objects by the full width at half maximum of the intensity point spread function is given by Equation (3-2)

$$
d_{\mathrm{FWHM}}=\frac{0.51 \cdot \lambda_{\mathrm{ex}}}{n \sin \alpha}=\frac{0.51 \cdot \lambda_{\mathrm{ex}}}{N A}
$$

with $\lambda_{\text {ex: }}$ excitation wavelength, $N A=n \sin \alpha$ : numerical aperture of the objective, $n$ : refractive index of the imaged medium and $\alpha$ : the opening angle of the objective. This leads to a resolution of a confocal microscope in the range of $200-400 \mathrm{~nm}$, if visible light is applied for excitation. By minimizing the pinhole diameter, the resolution can be enhanced up to $\sim 1.4$ times. However, this gain in resolution is accompanied by significant loss in detected intensity, which is unfavorable if a very high resolution is not required. ${ }^{[112]}$ 


\section{Experimental procedure}

SNARE-mediated single vesicle fusion of artificial proteoliposomes as well as chromaffin granules (CGs) with pore-spanning membranes (PSMs) was analyzed by means of dual color confocal laser scanning fluorescence microscopy. For this purpose, the upright LSM 710 (ZEISS, Jena, Germany) equipped with a water immersion objective WPlan-APOCHROMAT (63x, NA = 1.0, ZEISS, Jena, Germany) was used. By utilizing a spectral detector (photomultiplier tube, PMT), two channel recording in specific and distinct detection ranges was performed. As a fluorescent marker for the PSMs, Atto488-DPPE was inserted, while the artificial proteoliposomes were labeled with TxR-DPPE and the CGs with DiD-C 18 . Excitation wavelengths that were used for the individual fluorescent probe as well as the ranges for spectral separated detection are listed in Table 3-15.

Table 3-15 Excitation wavelengths and detection ranges for the fluorescent probes Atto488-DPPE, TxRDPPE and DiD-C 18 used for CLSM-measurements.

\begin{tabular}{lccc}
\hline & PSMs & Proteoliposomes & CGs \\
\hline Fluorescent probe & Atto488-DPPE & TxR-DPPE & DiD-C 18 \\
Excitation wavelength & $488 \mathrm{~nm}$ & $561 \mathrm{~nm}$ & $633 \mathrm{~nm}$ \\
Detection range & $495-545 \mathrm{~nm}$ & $610-700 \mathrm{~nm}$ & $640-729 \mathrm{~nm}$ \\
\hline
\end{tabular}

PSMs were prepared as described in Chapter 3.2.6 and positioned under the microscope. Proteoliposome suspension $(0.5-1.0 \mu \mathrm{L})$ or CG suspension $(0.5-1.0 \mu \mathrm{L})$ was carefully injected above the PSM patch in the focal plane and image acquisition was started. Time series of 2500 images with a frame rate of $8 \mathrm{~Hz}$, a resolution of $256 \times 256$ pixel $^{2}$ and a color depth of 16 bit were recorded. The area of the imaged PSM patch was always $\sim 40 \mathrm{x} 40 \mu \mathrm{m}^{2}$ and the detector pinhole was adjusted to a diameter of $300 \mathrm{~nm}$, decreasing the focal resolution, but allowing for better detection of vesicles above the PSM patch.

To investigate SNARE-mediated single vesicle fusion, time resolved fluorescence intensities from both channels (Atto488- and TxR-channel) were extracted. This was done in the first step by a threshold based localization of vesicles that docked to the PSM, followed by placing a region of interest (ROI) of $2 \times 2-5 \times 5$ pixel $^{2}$ on the center of mass of these vesicles and reading out the fluorescence intensities of both channels within this ROI. For this kind of analysis, the programs Matlab, Zen 2012 and ImageJ were applied, it is detailed in the dissertation of L. SCHWENEN. ${ }^{[101]}$ For identification of mobile vesicles and tracking, the software TrackNTrace was used. ${ }^{[113]}$ 


\subsubsection{Reflectometric interference spectroscopy (RIfS)}

Reflectometric interference spectroscopy (RIfS) is a biosensing technique which was used to monitor the binding of syt-1 doped small unilamellar vesicles (SUVs) to $\mathrm{PI}(4,5) \mathrm{P}_{2}$ containing solid supported membranes (SSMs). RIfS experiments were carried out by FRANZISKA DöRR during her bachelor thesis supervised from June to September 2016. Therefore, the theoretical background of this method as well as the experimental procedure are explained briefly.

\section{Theoretical background}

In RIfS, the interference of visible light is utilized to measure differences in optical thicknesses of thin films. With this technique, kinetics of binding processes at interfaces can be investigated without the need for additional labels but only the physical properties of light.

When light travels through a medium with the refractive index $n_{1}$ and hits the interface of a second optically transparent medium with the refractive index $n_{2}$ at an incidence angle $\alpha_{1}$, it will be partly refracted or reflected at this interface (Figure 3-21).

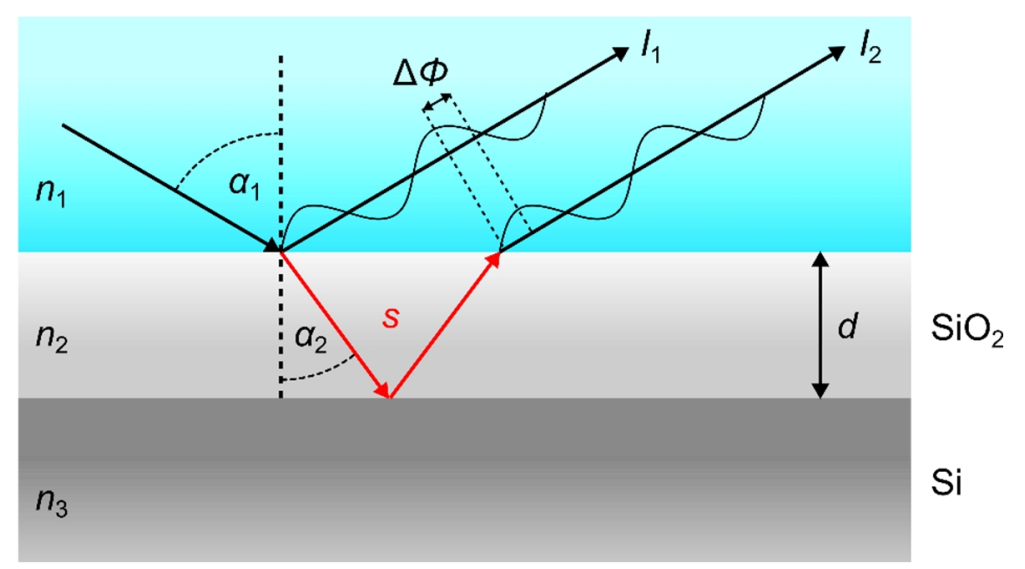

Figure 3-21 Schematic illustration of the light pathway in RIfS. Incoming light is partly reflected ( $\left.\mathrm{I}_{1}\right)$ or refracted $\left(\mathrm{I}_{2}\right)$ at the interface between two media with distinct refractive indices $\mathrm{n}_{1}$ and $\mathrm{n}_{2}$. The refracted part of the light is reflected while hitting an optically intransparent medium with the refractive index $n_{3}$, followed by reentering the medium $n_{1}$. This leads to an overlap of $I_{1}$ and $I_{2}$ causing constructive or destructive interference which depends on the phase difference $\Delta \phi$ between the two waves.

In case of $n_{1}<n_{2}$, part of the incoming light will be refracted towards the normal at the angle $\alpha_{2}$. When this refracted part passes now through the second medium and hits the surface of an optically intransparent third medium with the refractive index $n_{3}$, it will be nearly completely reflected. During reentering the first medium, the light will be refracted again, this time away from the normal at the angle $\alpha_{1}$. The additional traveling distance $s$ of the refracted part of the incoming light $\left(I_{2}\right)$ through the second medium causes a phase difference $\Delta \phi$ to the reflected 
part $\left(I_{1}\right) \cdot{ }^{[14]}[115]$ Here, $s$ of $I_{2}$ depends on the physical thickness $d$ of the second medium, on the angle of refraction $\alpha_{2}$ as well as on the refractive index $n_{2}$ and is given by Equation (3-3).

$$
s=\frac{2 \cdot n_{2} \cdot d}{\cos \alpha_{2}}
$$

For $\alpha_{1}=\alpha_{2}=0$ - the incoming light hits the surface perpendicular - Equation (3-3) can be simplified to:

$$
s=2 \cdot n_{2} \cdot d=2 \cdot O T
$$

The resulting product of the physical thickness $d$ and the refractive index $n_{2}$ is referred to as optical thickness $O T=n_{2} \cdot d$ of the second medium. Under perpendicular illumination, the connection between the phase shift $\Delta \phi$ and $O T$ can be described as done in Equation (3-5).

$$
\Delta \phi=\frac{2 \pi}{\lambda} \cdot O T
$$

Hence, for constructive interference, $\Delta \phi$ has to be a multiple of the wavelength $\lambda$, whereas destructive interference is observed in case of $\Delta \phi=\lambda / 2$. This leads to an interference pattern which depends on the wavelength used for illumination, on the physical thickness of the layer investigated and on its refractive index. One main criterion for the occurrence of interference is that the coherence length of the light must be larger than the optical path difference. If white light is used for illumination, this condition results in a physical thickness of the investigated layer of $0.5-10 \mu \mathrm{m}$ necessary to cause interference. The resulting intensity $R$ of the two reflected light rays $I_{1}$ and $I_{2}$ under perpendicular illumination can be expressed as shown in Equation (3-6), depending on $O T$ and $\lambda .^{[116]}$

$$
R(\lambda, O T)=\frac{r_{12}^{2}+r_{23}^{2}+2 \cdot r_{12} \cdot r_{23} \cdot \cos \left(\frac{4 \pi}{\lambda} \cdot O T\right)}{1+r_{12}^{2} \cdot r_{23}^{2}+r_{12} \cdot r_{23} \cdot \cos \left(\frac{4 \pi}{\lambda} \cdot \Delta \phi\right)}
$$

With $r$ being the Fresnel coefficient $r_{\mathrm{ij}}=\left(n_{\mathrm{i}}-n_{\mathrm{j}}\right) /\left(n_{\mathrm{i}}+n_{\mathrm{j}}\right)$ induced by the media's refractive indices $n_{\mathrm{i} / \mathrm{j}}$. Using Equation (3-6), the optical thickness $O T$ of the investigated layer can be calculated from a recorded reflectivity spectrum $R(\lambda)$ if the refractive indices $n$ of all involved media are known. 


\section{Experimental procedure}

RIfS was used to analyze binding of syt- 1 doped vesicles to $\mathrm{PI}(4,5) \mathrm{P}_{2}$ containing solid supported membranes (SSMs). For this purpose, SSMs with different amounts of $\mathrm{PI}(4,5) \mathrm{P}_{2}(0$, $1,2,5 \mathrm{~mol} \%)$ were prepared and syt- $1(\mathrm{p} / \mathrm{l}=1: 1000)$ was reconstituted into small and large unilamellar vesicles (SUVs and LUVs) using the protocol according to HERNANDEZ as described in (Chapter 3.2.5). For determination of binding affinities, proteoliposome suspension with a defined concentration was applied to a PI $(4,5) \mathrm{P}_{2}$ containing SSM within a closed loop, followed by rinsing in an open system. An observed increase in optical thickness $\Delta O T$ was assigned to vesicle binding to the $\mathrm{SSM}$ based on a syt-1/PI(4,5) $\mathrm{P}_{2}$ specific interaction. To verify syt-1/PI(4,5) $\mathrm{P}_{2}$ specificity, control experiments were performed by adding vesicles without protein to $\mathrm{PI}(4,5) \mathrm{P}_{2}$ containing membranes as well as by adding syt-1 doped proteoliposomes to SSMs without PI $(4,5) \mathrm{P}_{2}$. Experiments were carried out as follows:

- SSMs were prepared on hydrophilically functionalized silicon wafers with a $5 \mu \mathrm{m}$ silicon dioxide layer on top. This was achieved by spreading SUVs $(0.2 \mathrm{mg} / \mathrm{mL}$, composition cf. Table 3-16) in citrate buffer (20 mM Na-citrate, $50 \mathrm{~mm} \mathrm{KCl}, 0.1$ mMEDTA, $0.1 \mathrm{mM} \mathrm{NaN}_{3}$, $\mathrm{pH}$ 4.8) until a constant $O T$ was detected, followed by rinsing the system with SNARE buffer.

Table 3-16 Lipid composition of SUVs used for the preparation of SSMs for RIfS measurements.

\section{Lipid mixture for SSM preparation}

\begin{tabular}{cccccc}
\hline & PI(4,5) $\mathrm{P}_{2}$ & DOPC & POPE & POPS & Chol \\
$\mathbf{m o l} \%$ & $0-5$ & $45-50$ & 20 & 10 & 20 \\
\hline
\end{tabular}

- To verify comparable conditions between the measurements for different $\mathrm{PI}(4,5) \mathrm{P}_{2}$ contents, the proteoliposome concentration was determined indirectly via phosphate assay (Chapter 3.2.11) and a phosphate concentration of $c^{\prime}\left(\mathrm{PO}_{4}{ }^{3-}\right)=6.0 \mathrm{ng} / \mu \mathrm{L}$ was adjusted. The concentration-adjusted suspension was added to the SSM and time-resolved changes in optical thickness were measured ( $\triangle O T$ vs. $t$ ). Changes of the optical thickness $O T$ were converted into the changes in physical thickness $d$ according to Equation (3-4), assuming a refractive index for the lipid bilayer of $n_{\text {lipid }}=1.49 .{ }^{[117],[118]}$ 
Table 3-17 Lipid composition of syt-1 doped proteoliposomes used for RIfS measurements.

\section{Lipid mixture for proteoliposome preparation}

\begin{tabular}{cccccc}
\hline & TxR-DPPE & DOPC & POPE & POPS & Chol \\
$\mathbf{m o l} \%$ & 1 & 55 & 19 & 5 & 20 \\
\hline
\end{tabular}

For reflectivity measurements, an area of $1 \mathrm{~mm}^{2}$ of the wafer surface was illuminated by a tungsten halogen lamp and intensity spectra were recorded using a Nanocalc-2000 vis/NIR spectrometer (Ocean Optics, Largo, FL, USA). Data analysis and calculation of $O T$ was performed on the fly, whereby a Matlab-based script developed by MILENA STEPHAN ${ }^{[119]}$ and revised by INGO MEY was used. This software extracts the recorded reflectivity $R(\lambda, O T)$ between 500-700 nm and fits Equation (3-6) to the data to calculate OT. By this procedure, the changes in optical thickness $\triangle O T$ of a thin layer on top of the hydrophilically functionalized $\mathrm{SiO}_{2}$ surface can be recorded in a time-resolved manner on a sub-nanometer scale (Figure $3-22 \mathrm{C})$.

A

B
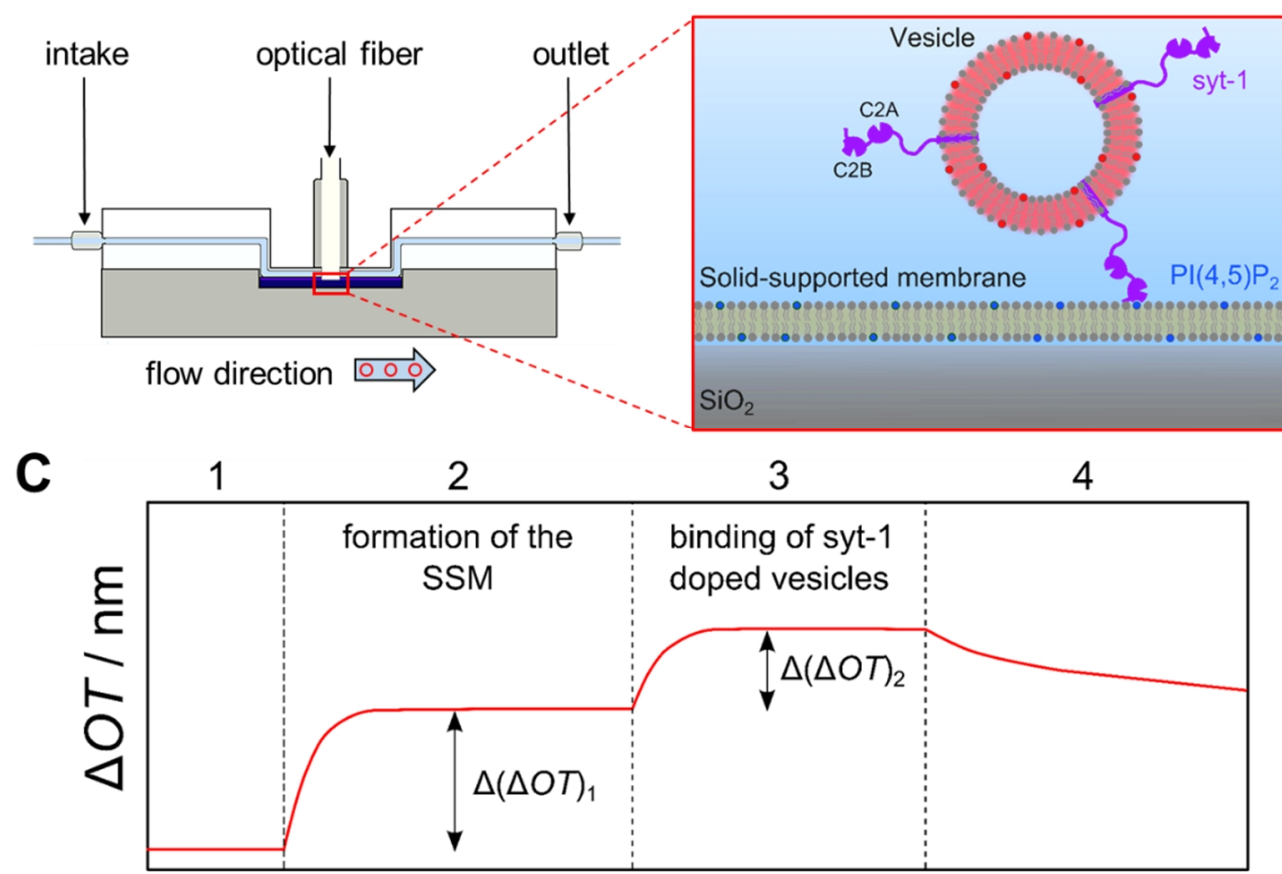

Time / $\mathrm{min}$

Figure 3-22 (A) Schematic illustration of the flow chamber for RIfS-measurements. (B) Close up of the schematic cross section of the measuring area. The $\mathrm{Ca}^{2+}$-independent, electrostatic interaction between the poly lysine stretch of syt-1's C2B domain (324-KKKK-327) and the anionic headgroup of PI(4,5) $\mathrm{P}_{2}$ mediates the binding of syt- 1 doped vesicles to $\mathrm{PI}(4,5) \mathrm{P}_{2}$ containing membranes. (C) Exemplary, schematic course of time-resolved $\Delta \mathrm{OT}$ during a RIfS-measurement. After recording a baseline (1), the addition of $\mathrm{PI}(4,5) \mathrm{P}_{2}$ containing SUVs causes the first increase in optical thickness $\left(\Delta \mathrm{OT}_{1}\right)$ due to the formation of a solid supported membrane (SSM) by spreading on top of the hydrophilically functionalized $\mathrm{SiO}_{2}$ surface (2). Subsequent addition of syt-1 doped vesicles lead to a second increase in optical thickness $\left(\Delta \mathrm{OT}_{2}\right)$ by binding to the $\mathrm{PI}(4,5) \mathrm{P}_{2}$ containing SSM (3). After reaching a constant value, reversibility of the syt-1/ $\mathrm{PI}(4,5) \mathrm{P}_{2}-$ binding is analyzed by rinsing the chamber with buffer (4). 



\section{$4 \quad$ Results}

SNARE-mediated fusion of two distinct lipid bilayers as it occurs for example prior to neurotransmitter release in a chemical synapse has been in focus of research for decades now. Many interesting and important observations were made but there is still no clear picture of the exact mechanism of neuronal fusion up till now.

To obtain a better resolution of this picture, a suited model system to study SNARE-mediated fusion under well-defined conditions in vitro can be helpful. A model system which resembles the natural situation, especially the membrane geometries found in a synaptic bouton quite well was recently developed in our laboratory. ${ }^{[43],[44],[45]}$ This in vitro approach is based on SNAREmediated fusion of two distinct artificial membrane systems - namely proteoliposomes and pore-spanning membranes (PSMs) - to observe and characterize fusion events on a single vesicle level. As this assay constitutes the basis for the investigation of SNARE-mediated single vesicle fusion performed in the context of this work, its main properties will be presented briefly in the following chapter.

\subsection{Basic properties of the artificial model system}

As a fusion machinery, the t-SNARE complex $\triangle \mathrm{N} 49$, comprised of SNAP25a, syx-1A (183288) and syb 2 (49-96), was reconstituted into PSMs and the v-SNARE syb 2 into liposomes. The reconstitution of both, syb 2 and $\Delta \mathrm{N} 49$, was performed according to the protocol of HERNANDEZ. ${ }^{[102]}$ It is based on co-micellization of detergent, lipids and proteins, followed by rapid dilution and size exclusion chromatography to remove the detergent and yields small unilamellar proteoliposomes (proteo-SUVs). These proteo-SUVs were the basis for the preparation of syb 2 containing large proteoliposomes (proteo-LUVs) via extrusion and $\Delta \mathrm{N} 49$ containing giant proteoliposomes (proteo-GUVs) via electroformation. By spreading these proteo-GUVs on hydrophilically functionalized porous substrates, $\triangle \mathrm{N} 49$ containing PSMs were prepared. The reconstitution and preparation protocols are detailed in Chapter 3.2.5.

As this model system was designed to be the basis for further enhancement and variation in conditions for the investigation of SNARE-mediated single vesicle fusion, it had to be thoroughly characterized in terms of functional and efficient SNARE reconstitution as well as protein and lipid mobility in PSMs. This was performed by and in collaboration with L. SCHWENEN and J. KUHLMANN and is summarized in Chapter 4.1.1 and Chapter 4.1.2. 


\subsubsection{Reconstitution of SNAREs into liposomes and pore-spanning membranes}

For the verification of successful and functional reconstitution of syb 2 into LUVs and $\Delta \mathrm{N} 49$ into GUVs, each step of the reconstitution protocol was analyzed in detail. In both cases, syb 2 and $\Delta \mathrm{N} 49$, the introduced molar protein to lipid ratio was $\mathrm{p} / \mathrm{l}=1: 500$. A lipid mixture of DOPC/POPE/POPS/cholesterol (5:2:1:2) was used, matching the lipid compositions found in nerve terminals quite well. ${ }^{[120]}$ To check for functional reconstitution of syb 2 into SUVs and LUVs as well as for $\triangle \mathrm{N} 49$ into SUVs and GUVs, vesicle-vesicle bulk fusion experiments were carried out as described in Chapter 3.3.3. SNARE specific fusion of the distinct vesicle populations was observed for proteo-SUVs as well as for proteo-LUVs and -GUVs as shown in Figure 4-1.

A

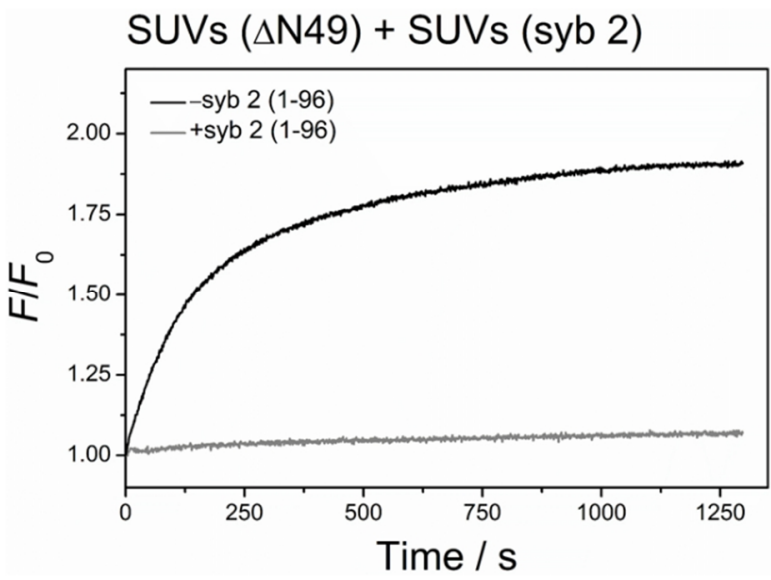

B

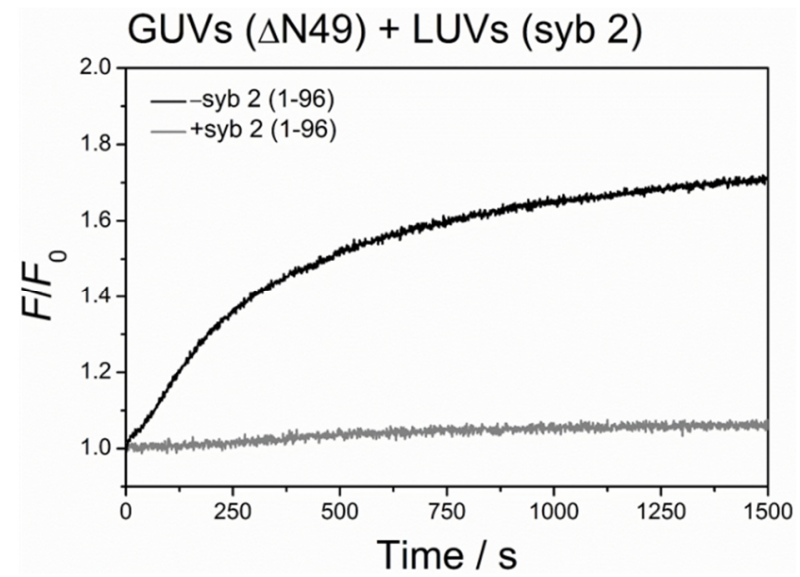

Figure 4-1 Time-resolved fluorescence intensity curves of the FRET-acceptor (TxR-DPPE) at $607 \mathrm{~nm}$ in vesicle-vesicle bulk fusion experiments while exciting the FRET-donor (Atto488-DPPE) at $495 \mathrm{~nm}$. An increase of the FRET-acceptor's fluorescence intensity indicates lipid mixing of the distinct vesicle populations upon fusion. (A) Bulk fusion experiment of SUVs ( $\triangle$ N49, Atto488-DPPE) with SUVs (syb 2, TxRDPPE) (black) and the corresponding control experiment, for which the $\triangle$ N49 doped SUVs were incubated with syb 2 (1-96) prior to fusion (grey). (B) Bulk fusion experiment of GUVs ( $\triangle$ N49, Atto488-DPPE) with LUVs (syb 2,TxR-DPPE) (black) and the corresponding control experiment, for which the $\Delta$ N49 doped GUVs were incubated with syb 2 (1-96) prior to fusion (grey).

Bulk fusion experiments, carried out after each step of the reconstitution protocol, were used for qualitative validation of functional SNARE reconstitution. A quantitative analysis of the reconstitution efficiencies for syb 2 into SUVs and LUVs as well as for $\triangle N 49$ into SUVs and GUVs was performed by J. KuHLMAnN. ${ }^{[90]}$ Those investigations indicated a $~ 90 \%$ reconstitution efficiency for both, syb 2 and $\Delta N 49$, into SUVs and LUVs, whereas the reconstitution efficiency for $\triangle \mathrm{N} 49$ into GUVs was found to be only $\sim 1 \%$. Nevertheless, functional reconstitution of SNAREs into both model systems (proteoliposomes and PSMs) was proven with these experiments, resulting in a SNARE density of $\sim 120 \Delta$ N49-complexes 
per $\mu \mathrm{m}^{2}$ GUV membrane $(\mathrm{p} / 1 \approx 1: 50000)$ and $\sim 500$ syb 2/proteoliposome $(\mathrm{p} / 1 \approx 1: 550)$. An average proteoliposome diameter of $240 \mathrm{~nm}$ and a mean lipid area of $0.65 \mathrm{~nm}^{2}$ was assumed for these calculations. ${ }^{[00]}$

\subsubsection{Mobility of SNAREs and lipids in pore-spanning membranes}

Pore-spanning membranes (PSMs) were prepared by spreading proteo-GUVs on top of hydrophilically functionalized porous substrates as detailed in Chapter 3.2.6. Since functional reconstitution of $\triangle \mathrm{N} 49$ into GUVs was proven (cf. Chapter 4.1.1), their functionality in PSMs can be assumed as well. As SNARE-mobility is prerequisite for the successful assembly of tight SNARE-bundles to induce fusion, the mobility of SNAREs and lipids in PSMs had to be analyzed. PSMs feature two distinct membrane areas, the solid supported part (s-PSM) on the rim and the free-standing part (f-PSM) within the pores (cf. Figure 4-2). Therefore, mobilities of SNAREs and lipids were determined in each of the parts individually.

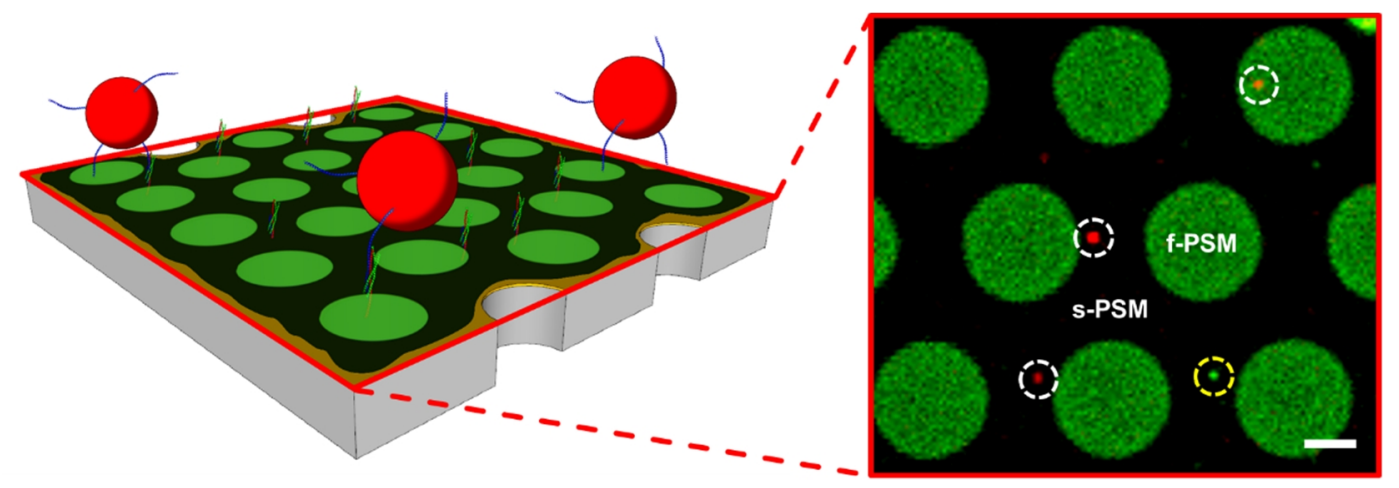

Figure 4-2 (left) Schematic illustration of a PSM patch, fluorescently labeled with Atto488-DPPE and doped with $\triangle$ N49. TxR-DPPE labeled proteoliposomes with reconstituted syb 2 are depicted in red. (right) Fluorescence micrograph of a PSM patch with docked proteo-LUVs (highlighted in dashed white circles) on both membrane areas, the solid supported part (s-PSM) and the free-standing part (f-PSM) as well as an already fused proteo-LUV (highlighted in a dashed yellow circle). (scale bar: $2 \mu \mathrm{m}$ ).

The diffusion behavior of SNAREs and lipids in f-PSMs were analyzed using fluorescence correlation spectroscopy (FCS), whereas the mobilities in s-PSMs had to be determined by indirect fluorescence recovery after photobleaching (FRAP). FCS experiments were partly performed by L. SCHWENEN ${ }^{[44],[101]}$ and the indirect FRAP measurements were carried out by J. KUHLMANN ${ }^{[90]}$. For the analysis of SNARE mobility, fluorescently labeled full length syx-1A (Alexa488-syx-1A) as well as two differently labeled sxy-1A transmembrane domains (Atto647N-syx-1A-TMD and Oregon Green (OG)-syx-1A-TMD) served as references. Lipid diffusion coefficients were determined for the fluorescent probes Atto488-DPPE and KK114DPPE. The obtained diffusion coefficients of SNAREs and lipids in the distinct membrane parts (s-PSM and f-PSM) are listed in Table 4-1. 
Table 4-1 Diffusion coefficients D of fluorescently labeled syx-1A, syx-1A-TMDs and DPPE in the two parts of pore-spanning membranes. Mobilities in the free-standing part (f-PSM) were determined via FCS, whereas the diffusion coefficients in the solid supported part (s-PSM) were obtained by indirect FRAP.

\begin{tabular}{|c|c|c|}
\hline & $D(\mathrm{f}-\mathrm{PSM}) / \mu^{2} \cdot \mathrm{s}^{-1}$ & $D(\mathrm{~s}-\mathrm{PSM}) / \mu \mathrm{m}^{2} \cdot \mathrm{s}^{-1}$ \\
\hline Alexa488-syx-1A & $2.3 \pm 0.5$ & \\
\hline OG-syx-1A-TMD & & $1.0 \pm 0.3^{*}$ \\
\hline Atto647N-syx-1A-TMD & $3.4 \pm 0.2 * *$ & \\
\hline Atto488-DPPE & $7.7 \pm 0.4 * *$ & $3.0 \pm 0.5^{*}$ \\
\hline KK114-DPPE & $7.4 \pm 0.3$ & \\
\hline
\end{tabular}

With these measurements, the mobility of lipids and SNAREs in both parts of a PSM was demonstrated, providing evidence that a continuous lipid bilayer is formed. Furthermore, from the experimental verification that syx-1A is mobile in both parts of the PSM, it can be concluded that the $\Delta$ N49-complex, which is anchored in the PSM via the syx-1A-TMD, exhibits approximately the same diffusion coefficients. However, it should be noted that the immobile fraction of syx-1A in the s-PSM was not determinable by FRAP owing to the quenched fluorescence by the underlying gold layer. Nevertheless, it can be concluded from FCS, indirect FRAP and FLIP (fluorescence loss in photobleaching) experiments that the requirement of SNARE-mobility in the model system of PSMs is fulfilled. [44],[45],[90],[101]

\subsubsection{Characteristics of single vesicle fusion events}

Pore-spanning membranes fulfill many prerequisites a model system should provide for the investigation of SNARE-mediated single vesicle fusion, e.g. protein and lipid mobility as well as a planar and freestanding lipid bilayer part (cf. Chapter 4.1.1 and Chapter 4.1.2). To monitor single vesicle fusion events between $\triangle \mathrm{N} 49$ containing PSMs doped with Atto488-DPPE and syb 2 containing LUVs labeled with TxR-DPPE, dual color confocal fluorescence microscopy was applied. The recorded time series clearly demonstrate that SNARE-mediated membrane fusion occurs between PSMs and proteo-LUVs and that key steps like docking, intermediate fusion and full fusion can be assigned. The time-resolved intensity traces of an exemplary fusion event which captures all important observables that were extracted to analyze SNAREmediated fusion on the single particle level is shown in Figure 4-3. The docking of the proteoLUV to the PSM is characterized by a sharp increase of fluorescence intensity in the red channel (TxR-DPPE fluorescence), whereas the fluorescence intensity in green channel (Atto488-DPPE 
fluorescence) is not affected during this fusion state. At the onset of fusion, lipid mixing takes place between the LUV membrane and the PSM. This leads to a simultaneous flux of Atto488DPPE from the PSM into LUV membrane and vice versa for TxR-DPPE. Owing to the fact that Atto488 (donor) and TxR (acceptor) is a FRET-pair, the start of lipid mixing is indicated by a FRET-induced dip in the green channel and a simultaneous sharp and short increase in the red channel. The following increase in the green channel is evoked by the constant influx of Atto488-DPPE into the LUV membrane leading to a saturation level. At the same time, TxRDPPE radially distributes into the PSM which is characterized by the synchronous decrease in the red channel. Such an increase in the green channel to a constant saturation level accompanied by a simultaneous decrease in the red channel to an intensity level between the docking and the baseline level was interpreted as an intermediate fusion state with a stable $\Omega$-shaped post-fusion structure - probably hemifusion. The second FRET followed by a decrease in both fluorescence intensities indicated full fusion and results in the complete merger of the $\Omega$-shaped post-fusion structure of the LUV into the PSM.

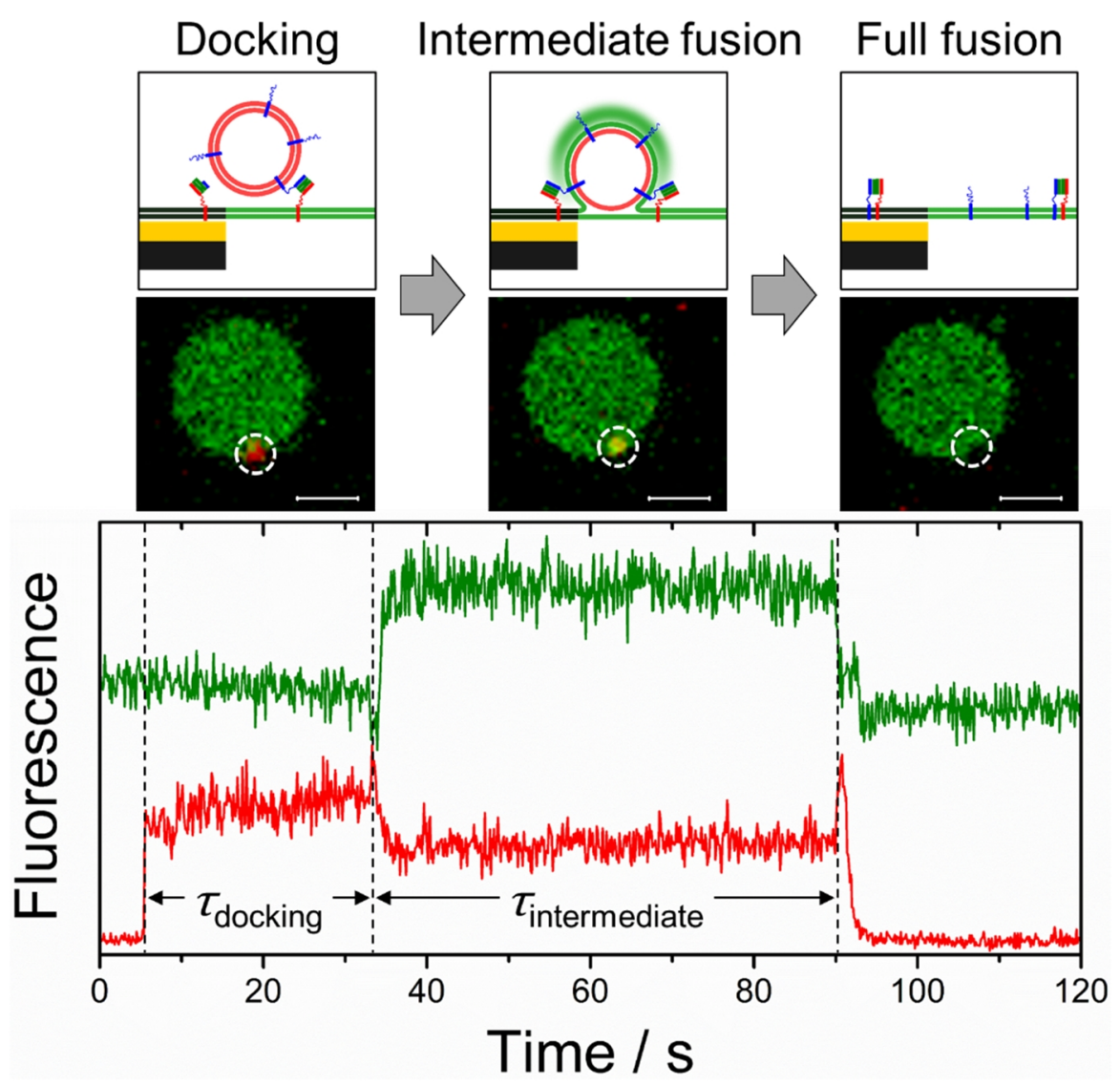

Figure 4-3 (bottom) Time-resolved fluorescence intensity traces of a TxR-DPPE doped vesicle with reconstituted syb 2 (red) fusing with an Atto488-DPPE labeled PSM containing $\Delta$ N49 (green). Key steps of single vesicle fusion events like docking, intermediate fusion as well as full fusion, can unambiguously be assigned to the time dependent intensities in both fluorescence channels. (top) Merged fluorescence micrographs of both channels corresponding to vesicle docking, intermediate fusion with an $\Omega$-shaped postfusion structure and full fusion (from left to right; scale bar: $2 \mu \mathrm{m}$ ). Above the fluorescence micrographs, schematic illustrations for each fusion state are depicted. 
Time-resolved fluorescence intensity traces of each individual proteoliposome that docked to the PSM within the recorded time series of $305 \mathrm{~s}$ ( $\triangleq 2500$ frames) were extracted with a semiautomated Matlab software written by B. GEIL and is detailed in the dissertation of L. SCHWENEN. ${ }^{[101]}$ The read out time traces allowed to class each detected event with either a full fusion, a stalled intermediate fusion or a stalled docking event depending on the fusion state that was reached until the end of the time window of observation. Moreover, the lifetimes of the docking state $\tau_{\text {docking }}$ and the intermediate fusion state $\tau_{\text {intermediate }}$ were extracted (cf. Figure 4-3) - if ascertainable.

SNARE-specificity of fusion was verified by control experiments, in which the $\Delta N 49$, incorporated into PSMs, was blocked by syb 2 (1-96) prior to proteo-LUV addition. In this case, neither fusion nor docking occurred. The same accounts for experiments in the absence of SNAP25a, where only syx-1A was reconstituted into the PSM.

\subsection{Impact of PI $(4,5) P_{2}$ on SNARE-mediated fusion}

The establishment of pore-spanning membranes and proteoliposomes as a model system to investigate neuronal SNARE-mediated fusion in vitro constituted the basis for further increase the complexity towards a more physiological model. For this purpose, the phosphoinositide $\mathrm{PI}(4,5) \mathrm{P}_{2}$ became the focus of attention as it accounts for a rapidly growing list of cellular functions and among these are membrane targeting as well as endo- and exocytosis. ${ }^{[4]}$ From the findings of VAN DEN BOGAART and coworkers that PI $(4,5) \mathrm{P}_{2}$ is the dominant inner leaflet lipid in clustered microdomains of syx-1A in the plasma membrane of PC12 cells, ${ }^{[51]}$ the question arose whether integration of $\mathrm{PI}(4,5) \mathrm{P}_{2}$ into the PSM's lipid composition would affect SNARE-mediated fusion in our model system. To this end, the PI(4,5) $\mathrm{P}_{2}$ content in the PSM was gradually increased from $1 \mathrm{~mol} \%$ to $2 \mathrm{~mol} \%$ to $5 \mathrm{~mol} \%$ to analyze its impact on SNAREmediated single vesicle fusion.

\subsubsection{Integration of $1 \mathrm{~mol} \% \mathrm{PI}(4,5) \mathrm{P}_{2}$}

As a first step, a PI(4,5) $\mathrm{P}_{2}$ content of $1 \mathrm{~mol} \%$ was adjusted in the lipid films (Table 4-2) used for the reconstitution of $\Delta \mathrm{N} 49(\mathrm{p} / \mathrm{l}=1: 500)$ into proteo-SUVs by the co-micellization method. The resulting proteo-SUVs were dried, rehydrated in sucrose solution and submitted to electroformation to obtain proteo-GUVs. The obtained proteo-GUVs, in turn, were spread on hydrophilically functionalized porous substrates yielding PSM patches with reconstituted $\Delta$ N49 
and a $\mathrm{PI}(4,5) \mathrm{P}_{2}$ content of $1 \mathrm{~mol} \%$. The lipid mixture used for the reconstitution of syb 2 into proteo-SUVs $(\mathrm{p} / \mathrm{l}=1: 500)$ that constituted the basis for proteo-LUV preparation via extrusion is shown in Table 4-2. The corresponding reconstitution and preparation protocols are detailed in Chapter 3.2.5 and Chapter 3.2.6.

Table 4-2 Composition of the lipid films used for the reconstitution of $\Delta$ N49 and syb 2 into proteo-SUVs. The amounts of lipids are stated in mol\%.

\begin{tabular}{cccccccc}
\hline & PI(4,5)P & DOPC & POPE & POPS & Chol & $\begin{array}{c}\text { Atto488 } \\
\text {-DPPE }\end{array}$ & $\begin{array}{c}\text { TxR- } \\
\text { DPPE }\end{array}$ \\
\hline $\mathbf{\Delta N 4 9}$ & 1 & 49 & 19 & 10 & 20 & 1 & -- \\
syb 2 & -- & 50 & 19 & 10 & 20 & -- & 1 \\
\hline
\end{tabular}

To verify functional reconstitution, vesicle-vesicle bulk fusion experiments were performed after each step of the reconstitution protocol on the path towards investigation of single vesicle fusion on PSMs. Figure 4-4A shows the normalized and time-resolved increase of the FRETacceptor's (TxR-DPPE) intensity at $607 \mathrm{~nm}$ as the mean of 8 bulk fusion experiments of proteoSUVs ( $\triangle$ N49) with proteo-SUVs (syb 2). As a kinetic parameter, the time needed to reach $50 \%$ of the total intensity was determined to be $\tau_{1 / 2}=128 \pm 11 \mathrm{~s}$. In addition, vesicle-vesicle bulk fusion experiments of proteo-GUVs $(\Delta \mathrm{N} 49)$ with proteo-LUVs (syb 2) were carried out (Figure 4-4B). The mean increase of 6 measurements resulted in an average value for $\tau_{1 / 2}$ of $125 \pm 24 \mathrm{~s}$.

A

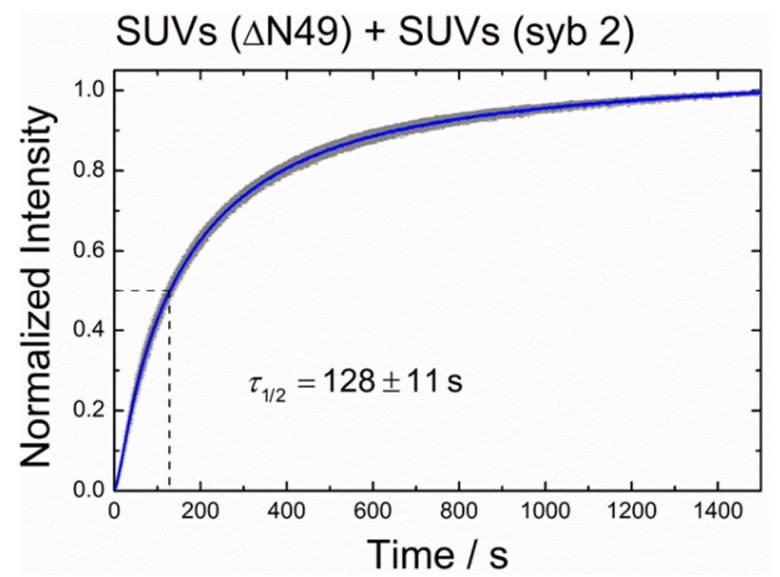

B

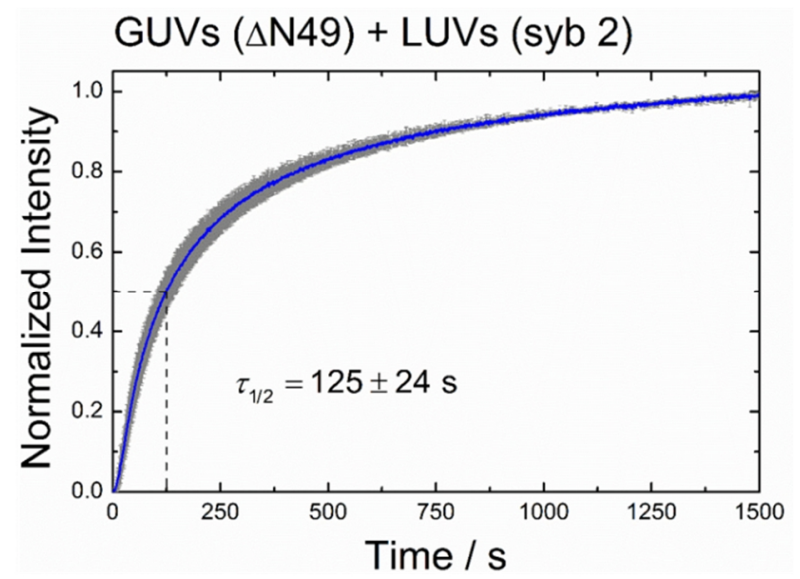

Figure 4-4 Normalized and time-resolved increase of the FRET-acceptor's (TxR-DPPE) fluorescence intensity in SNARE-mediated vesicle-vesicle bulk fusion experiments (blue line). Error bars are depicted in grey. (A) Average curve of 8 bulk fusion experiments of proteo-SUVs $(\Delta N 49)$ with proteo-SUVs (syb 2) leading to a value for $\tau_{1 / 2}$ of $128 \mathrm{~s}$. (B) Average curve of 6 bulk fusion experiments of proteo-GUVs $(\Delta N 49)$ with proteo-LUVs (syb 2) leading to a value for $\tau_{1 / 2}$ of $125 \mathrm{~s}$.

After the confirmation of functional reconstitution of SNARE-proteins into GUVs and LUVs, the $\triangle$ N49 doped GUVs were utilized for PSM preparation to analyze SNARE-mediated fusion 
on a single vesicle level. In 27 recorded time series on different PSM patches from 4 individual SNARE reconstitutions, 537 proteo-LUVs were detected and evaluated with the regard on their fusion behavior. This concerned, in a first step, the categorization of each detected event into the fusion state reached until the end of the recorded time series. Here, the fusion states were categorized into stalled docking, stalled intermediate fusion and full fusion (cf. Figure 4-3). The classification of the events into fusion states was conducted separately for each time series followed by the calculation of the respective percentages. The analysis resulted in a mean fusion efficiency of $79 \pm 5 \%$. This means that in average $21 \pm 3 \%$ of the vesicles that docked to the PSM with a $\mathrm{PI}(4,5) \mathrm{P}_{2}$ content of $1 \mathrm{~mol} \%$ were stalled in the docking state and did not proceed to fusion within the time window of observation. Among the $79 \%$ of the vesicles that fused with the PSM, $47 \pm 6 \%$ proceeded to full fusion, whereas $53 \pm 5 \%$ remained in an intermediate fusion state. The resulting statistics concerning fusion states of proteo-LUVs on PSMs with a $\mathrm{PI}(4,5) \mathrm{P}_{2}$ content of $1 \mathrm{~mol} \%$ are depicted in Figure 4-5.

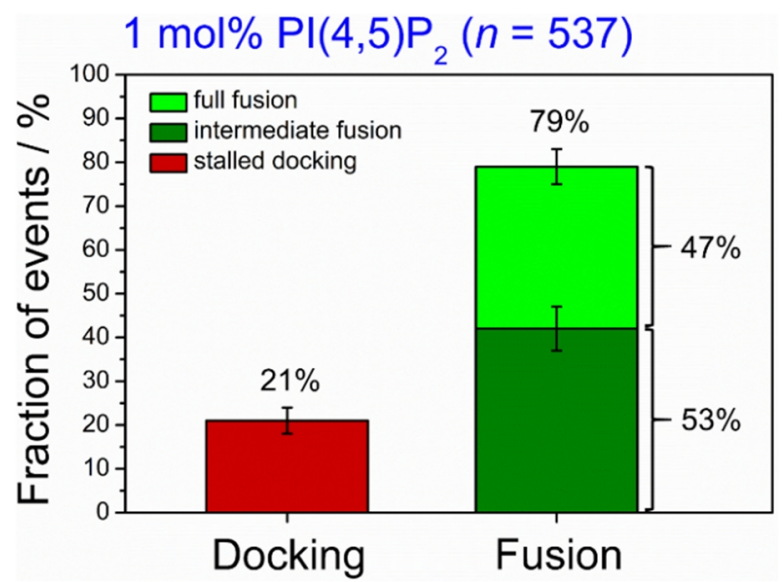

Figure 4-5 Statistical analysis of the fusion behavior of 537 detected proteo-LUVs (syb 2) docked to PSMs $(\triangle \mathrm{N} 49)$ with a $\mathrm{PI}(4,5) \mathrm{P}_{2}$ content of $1 \mathrm{~mol} \%$. The underlying data was extracted from 27 recorded time series on different PSM patches of 4 individual SNARE reconstitutions. The depicted values for the fraction of events represent the mean percentage obtained by a separate evaluation of the fusion states in each recorded time series resulting in a fusion efficiency of $79 \pm 5 \%$. The standard deviation from the resulting mean percentage of each fusion state is depicted as error bar (black).

Besides the categorization of fusion events, the lifetime of the docking state $\tau_{\text {docking }}$ was extracted from the intensity traces of 427 proteo-LUVs on PSMs doped with 1 mol\% PI(4,5) $\mathrm{P}_{2}$. Binning of the data according to the FREEDMAN-DIACONIS rule ${ }^{[121]}$ resulted in the histogram shown in Figure 4-6A. A log-normal distribution (Equation (4-1)) was fitted to the histogram of binned numbers of the determined docking lifetimes $N\left(\tau_{\text {docking }}\right)$

$$
N\left(\tau_{\text {docking }}\right)=\frac{A}{\sqrt{2 \pi} \cdot \sigma \cdot \tau_{\text {docking }}} \cdot \exp \left(-\frac{\left(\ln \left(\tau_{\text {docking }}\right)-\mu\right)^{2}}{2 \sigma^{2}}\right),
$$


with the variable parameters $A$ : area under the curve, $\mu$ : expectation and $\sigma^{2}$ : variance. The maximum of Equation (4-1) is called mode and describes the value for $\tau_{\text {docking }}$ that is most likely to be observed. The mode $\tau_{\text {docking }}^{\max }$ was calculated using Equation (4-2).

$$
\tau_{\text {docking }}^{\max }=\exp \left(\mu-\sigma^{2}\right)
$$

As a parameter to describe the broadness of the distribution of $N\left(\tau_{\text {docking }}\right)$ the full width at half maximum (FWHM) was determined. This analysis of the determined lifetimes of the docking state for proteo-LUVs on PSMs with a $\mathrm{PI}(4,5) \mathrm{P}_{2}$ content of $1 \mathrm{~mol} \%$ resulted in a value for $\tau_{\text {docking }}^{\max } 38 \pm 3 \mathrm{~s}$ and a FWHM of $47 \mathrm{~s}$ (Figure 4-6A).

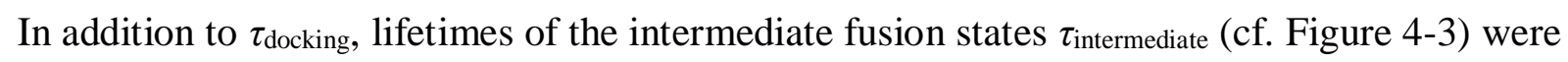
extracted from the intensity traces of 212 events which proceeded to full fusion within the time window of observation. To the resulting histogram of the binned numbers of $\tau_{\text {intermediate }}$ (Figure 4-6B), the mono exponential Equation (4-3) was fitted

$$
N\left(\tau_{\text {intermediate }}\right)=N_{0} \cdot \exp \left(-k \cdot \tau_{\text {intermediate }}\right)
$$

with $N_{0}$ : total number of intermediate fusion states that proceeded to full fusion and $k$ : the rate constant of the decay of the intermediate fusion state. From the resulting rate constant $k$, the average lifetime of the intermediate fusion state $\bar{\tau}_{\text {intermediate }}$ was calculated as the reciprocal.

A

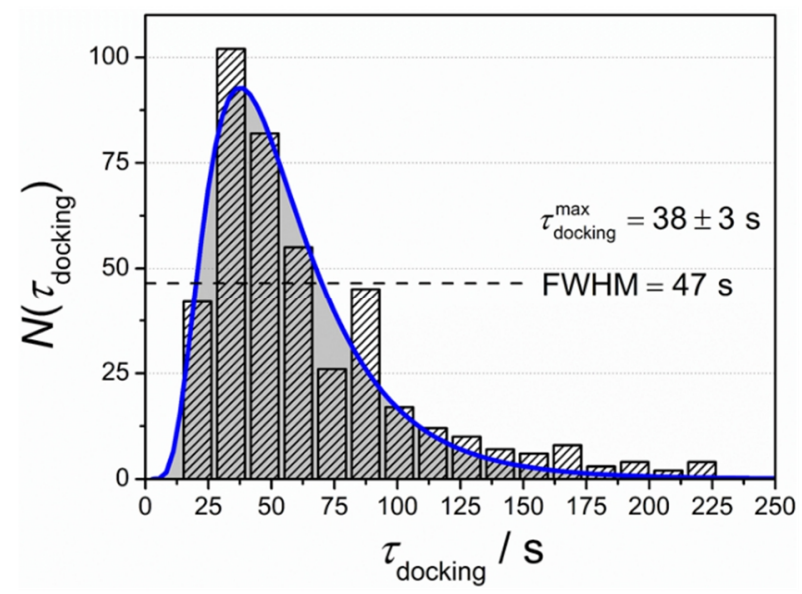

B

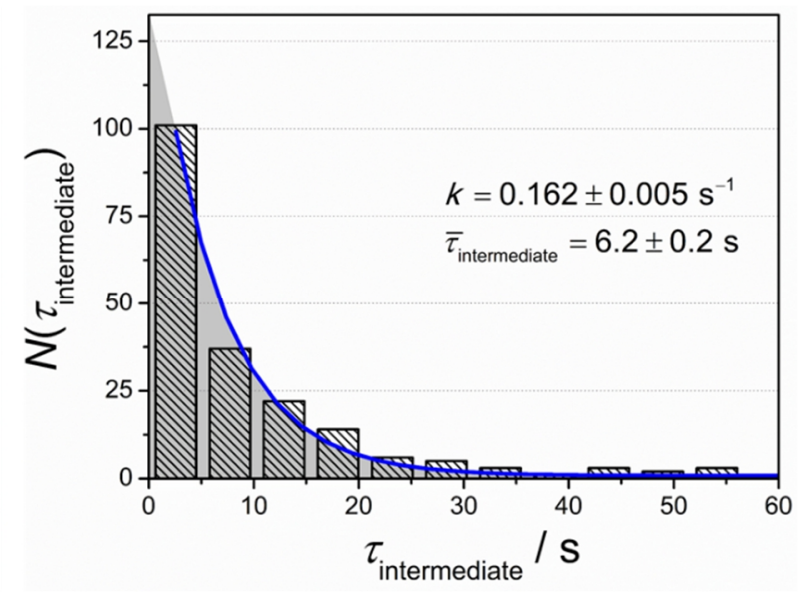

Figure 4-6 (A) Histogram of lifetimes of the docking state $\tau_{\text {docking }}$ of 427 proteo-LUVs on PSMs with a $\mathrm{PI}(4,5) \mathrm{P}_{2}$ content of $1 \mathrm{~mol} \%$. Fitting of a log-normal distribution (Equation (4-1)) to the data resulted in a maximum value for the docking lifetime of $38 \mathrm{~s}$ and a FWHM of $47 \mathrm{~s}$. (B) Histogram of lifetimes of the intermediate fusion state $\tau$ intermediate of a total number of 212 detected events on PSMs doped with $1 \mathrm{~mol} \%$ $\mathrm{PI}(4,5) \mathrm{P}_{2}$. Fitting of a mono exponential decay (Equation (4-3)) to the data resulted in a rate constant $\mathrm{k}$ for the decay of the intermediate fusion state of $0.162 \mathrm{~s}^{-1}$ and an average lifetime $\bar{\tau}_{\text {intermediate }}$ of $6.2 \mathrm{~s}$. 
The analysis of the obtained values for $\tau_{\text {intermediate }}$ yielded a rate constant $k$ for the decay of the intermediate fusion state of $0.162 \pm 0.005 \mathrm{~s}^{-1}$ and an average lifetime $\bar{\tau}_{\text {intermediate }}$ of this fusion state of $6.2 \pm 0.2 \mathrm{~s}$ before the onset of collapse of the proteo-LUV post fusion structure $(\Omega$ shape) into the PSM.

\subsubsection{Integration of $2 \mathrm{~mol} \% \mathrm{PI}(4,5) \mathrm{P}_{2}$}

As a second step, a PI(4,5) $\mathrm{P}_{2}$ content of 2 mol\% was adjusted in the lipid films (Table 4-3) used for the reconstitution of $\Delta \mathrm{N} 49$ into proteo-SUVs $(\mathrm{p} / \mathrm{l}=1: 500)$. Following the preparation protocol detailed in Chapter 3.2.6, $\Delta \mathrm{N} 49$ containing PSMs with a $\mathrm{PI}(4,5) \mathrm{P}_{2}$ of $2 \mathrm{~mol} \%$ were prepared.

Table 4-3 Composition of the lipid films used for $\Delta$ N49 reconstitution into proteo-SUVs that constituted the basis for proteo-GUV and PSM preparation. The amounts of lipids are stated in mol\%.

\begin{tabular}{ccccccc}
\hline PI(4,5)( $\mathbf{2}_{\mathbf{2}}$ & DOPC & POPE & POPS & Chol & $\begin{array}{c}\text { Atto488- } \\
\text { DPPE }\end{array}$ \\
\hline $\mathbf{\Delta N 4 9}$ & 2 & 48 & 19 & 10 & 20 & 1 \\
\hline
\end{tabular}

The lipid mixture used for the reconstitution of syb 2 into proteo-SUVs $(\mathrm{p} / \mathrm{l}=1: 500)$ that were needed for proteo-LUV preparation remained the same and is shown in Table 4-2. Again, vesicle-vesicle bulk fusion experiments were performed to verify SNARE functionality after each preparation step. Figure 4-7A shows the normalized and time-resolved increase of the FRET-acceptor's (TxR-DPPE) intensity at $607 \mathrm{~nm}$ as the mean of 7 bulk fusion experiments of proteo-SUVs ( $\triangle$ N49) with proteo-SUVs (syb 2) resulting in a value for $\tau_{1 / 2}$ of $122 \pm 13 \mathrm{~s}$. Vesicle-vesicle bulk fusion experiments of proteo-GUVs $(\Delta \mathrm{N} 49)$ with proteo-LUVs (syb 2$)$ were carried out as well (Figure 4-7B). The mean increase of 7 measurements resulted in an average value for $\tau_{1 / 2}$ of $154 \pm 24 \mathrm{~s}$. 
A

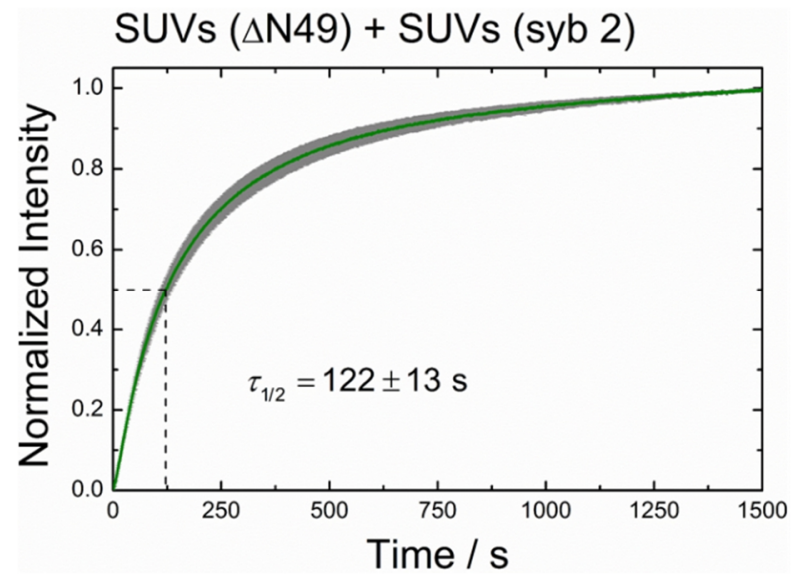

B

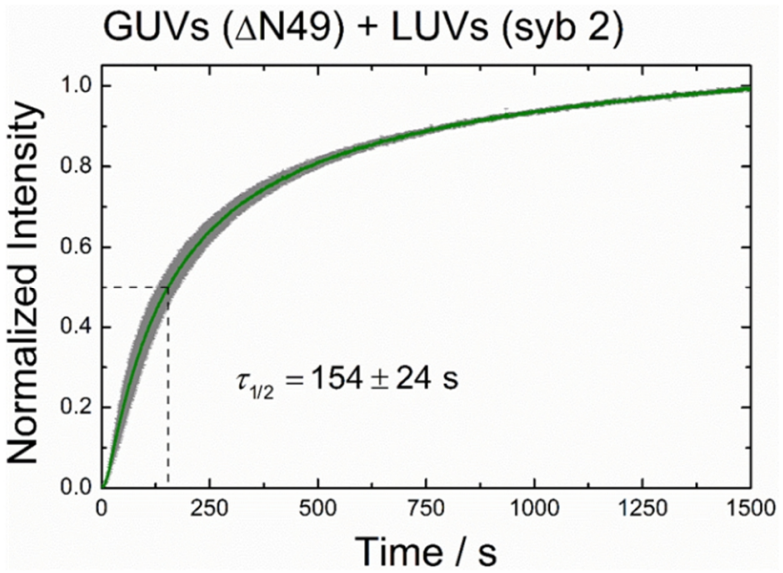

Figure 4-7 Normalized and time-resolved increase of the FRET-acceptor's (TxR-DPPE) fluorescence intensity in SNARE-mediated vesicle-vesicle bulk fusion experiments ( green line). Error bars are depicted in grey. (A) Average curve of 7 bulk fusion experiments of proteo-SUVs ( $\Delta$ N49) with proteo-SUVs (syb 2) leading to a value for $\tau_{1 / 2}$ of $122 \mathrm{~s}$. (B) Average curve of 7 bulk fusion experiments of proteo-GUVs $(\Delta N 49)$ with proteo-LUVs (syb 2) leading to a value for $\tau_{1 / 2}$ of $154 \mathrm{~s}$.

After the confirmation of functional reconstitution of SNARE-proteins into GUVs and LUVs, the $\triangle \mathrm{N} 49$ doped GUVs were utilized for PSM preparation to investigate SNARE-mediated fusion on a single vesicle level. In 23 recorded time series on different PSM patches of 2 individual SNARE reconstitutions, 994 proteo-LUVs were detected that docked to PSMs with a $\mathrm{PI}(4,5) \mathrm{P}_{2}$ content of $2 \mathrm{~mol} \%$ and evaluated concerning their fusion behavior. The separate analysis of the 23 different time series led to a remarkable mean for the observed fusion efficiency of $92 \pm 4 \%$. Only $8 \pm 2 \%$ of the vesicles that docked to the PSM were stalled in the docking state and did not proceed to fusion within the time window of observation. Among the $92 \%$ of the vesicles that fused with the PSM, in average $48 \pm 4 \%$ proceeded to full fusion, whereas $52 \pm 4 \%$ remained in an intermediate fusion state. The resulting statistics of this analysis of the fusion states are depicted in a bar diagram in Figure 4-8. 


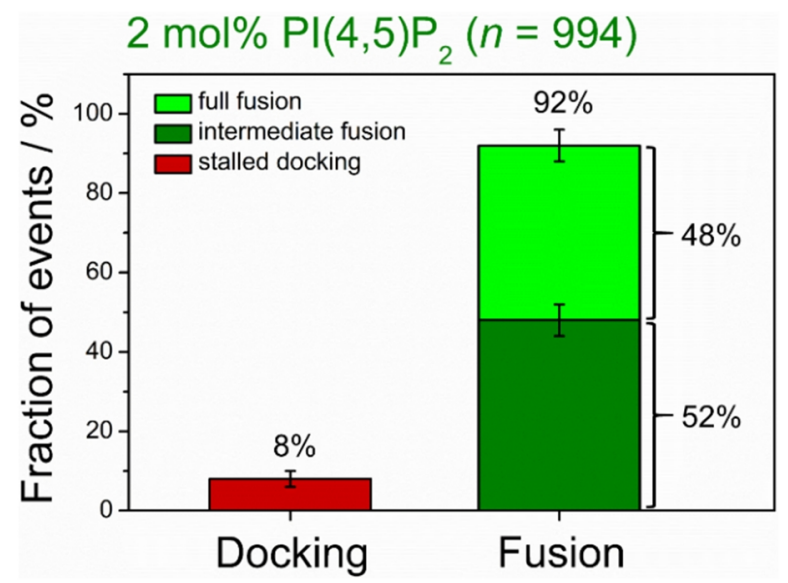

Figure 4-8 Statistical analysis of the fusion behavior of 994 detected proteo-LUVs (syb 2) docked to PSMs $(\Delta \mathrm{N} 49)$ with a $\mathrm{PI}(4,5) \mathrm{P}_{2}$ content of $2 \mathrm{~mol} \%$. The underlying data was extracted from 23 recorded time series on different PSM patches of 2 individual SNARE reconstitutions. The depicted values for the fraction of events represent the mean percentage obtained by a separate evaluation of the fusion states in each recorded time series resulting in a fusion efficiency of $92 \pm 4 \%$. The standard deviation from the resulting mean percentage of each fusion state is depicted as error bar (black).

Besides classification of the single vesicle fusion events, lifetimes of the docking state of 769 proteo-LUVs on PSMs doped with $2 \mathrm{~mol} \% \mathrm{PI}(4,5) \mathrm{P}_{2}$ were determined. Fitting of Equation (4-1) to the histogram of $N\left(\tau_{\text {docking }}\right)$ resulted in a value for $\tau_{\text {docking }}^{\max } 44 \pm 1 \mathrm{~s}$ (Figure $4-9 \mathrm{~A}$ ) and a FWHM of $55 \mathrm{~s}$. Further, lifetimes of the intermediate fusion state $\tau_{\text {intermediate }}$ of 425 single vesicle fusion events were extracted, a histogram of $N\left(\tau_{\text {intermediate }}\right)$ is shown in Figure 4-9B. By fitting a mono exponential decay (Equation (4-3)) to the data, a rate constant $k$ for the decay of the intermediate fusion state of $0.147 \pm 0.018 \mathrm{~s}^{-1}$ and the corresponding average lifetime $\bar{\tau}_{\text {intermediate }}$ of $6.8 \pm 0.8 \mathrm{~s}$ were obtained.

A

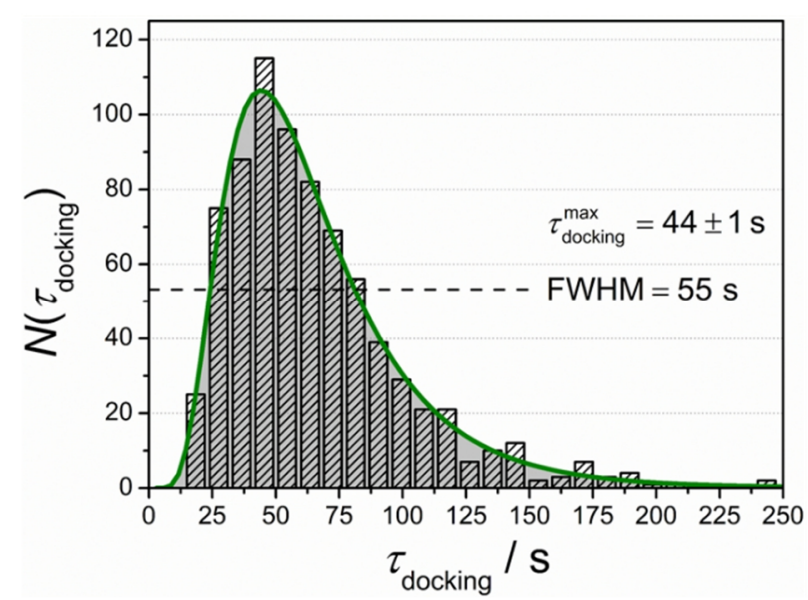

B

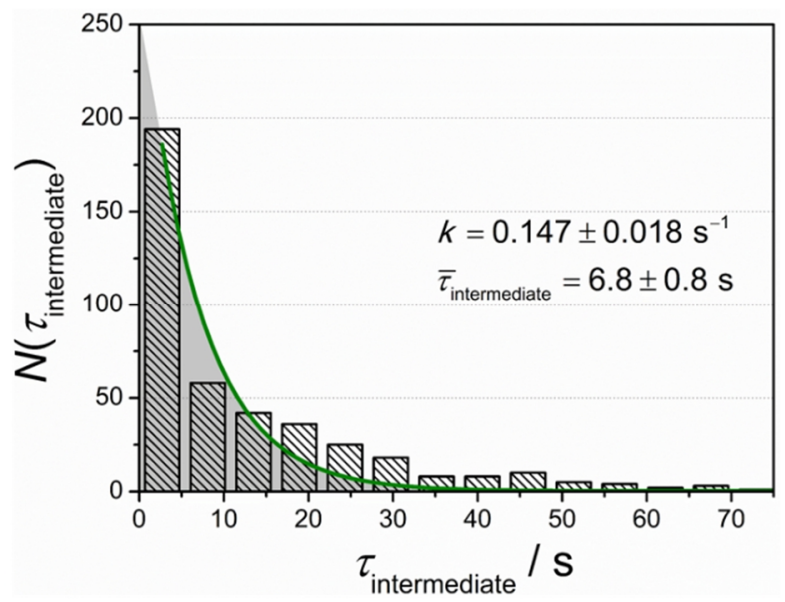

Figure 4-9 (A) Histogram of lifetimes of the docking state $\tau_{\text {docking }}$ of 769 proteo-LUVs on PSMs with a $\mathrm{PI}(4,5) \mathrm{P}_{2}$ content of $2 \mathrm{~mol} \%$. Fitting of a log-normal distribution (Equation (4-1)) to the data resulted in a maximum value for the docking lifetime of $44 \mathrm{~s}$ and a FWHM of $55 \mathrm{~s}$. (B) Histogram of lifetimes of the

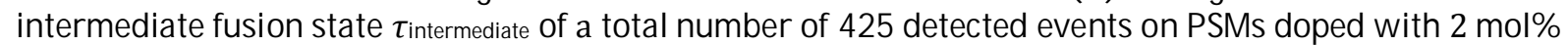
$\mathrm{PI}(4,5) \mathrm{P}_{2}$. Fitting of a mono exponential decay (Equation (4-3)) to the data resulted in a rate constant $\mathrm{k}$ for the decay of the intermediate fusion state of $0.147 \mathrm{~s}^{-1}$ and an average lifetime $\bar{\tau}_{\text {intermediate }}$ of $6.8 \mathrm{~s}$. 


\subsubsection{Integration of $5 \mathrm{~mol} \% \mathrm{PI}(4,5) \mathrm{P}_{2}$}

As a third step, after investigation of single vesicle fusion on PSMs with a $\mathrm{PI}(4,5) \mathrm{P}_{2}$ content of 1 and $2 \mathrm{~mol} \%$, the PI(4,5) $\mathrm{P}_{2}$ content was increased to $5 \mathrm{~mol} \%$. The composition of the lipid films used for the reconstitution of $\Delta \mathrm{N} 49$ into proteo-SUVs $(\mathrm{p} / \mathrm{l}=1: 500)$ is listed in Table 4-4. The obtained proteo-SUVs were dried, rehydrated in sucrose solution and submitted to electroformation to yield proteo-GUVs for PSM preparation.

Table 4-4 Composition of the lipid films used for $\Delta$ N49 reconstitution into proteo-SUVs that constituted the basis for proteo-GUV and PSM preparation. The amounts of lipids are stated in mol\%.

\begin{tabular}{ccccccc}
\hline & PI(4,5)P & DOPC & POPE & POPS & Chol & $\begin{array}{c}\text { Atto488- } \\
\text { DPPE }\end{array}$ \\
\hline $\mathbf{\Delta N 4 9}$ & 5 & 45 & 19 & 10 & 20 & 1 \\
\hline
\end{tabular}

The lipid mixture used for the reconstitution of syb 2 into proteo-SUVs $(p / 1=1: 500)$ for the subsequent preparation of proteo-LUVs remained the same and is shown in Table 4-2. In case of a $\mathrm{PI}(4,5) \mathrm{P}_{2}$ content of $5 \mathrm{~mol} \%$, vesicle-vesicle bulk fusion experiments were performed as well to confirm the preserved functionality of the SNAREs after each step of the reconstitution protocol. Figure 4-10A shows the normalized and time-resolved increase of the FRETacceptor's (TxR-DPPE) intensity at $607 \mathrm{~nm}$ as an average curve of 7 bulk fusion experiments of proteo-SUVs ( $\triangle \mathrm{N} 49)$ with proteo-SUVs (syb 2) leading to a value for $\tau_{1 / 2}$ of $114 \pm 6 \mathrm{~s}$. In vesicle-vesicle bulk fusion experiments of proteo-GUVs ( $\triangle$ N49) with proteo-LUVs (syb 2) (Figure 4-10B), the average curve of 6 measurements resulted in a value for $\tau_{1 / 2}$ of $144 \pm 45 \mathrm{~s}$.

A

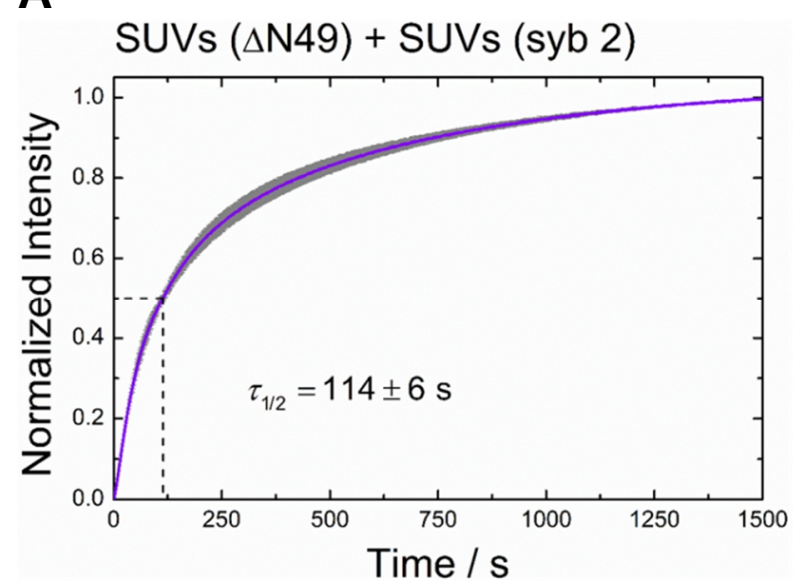

B

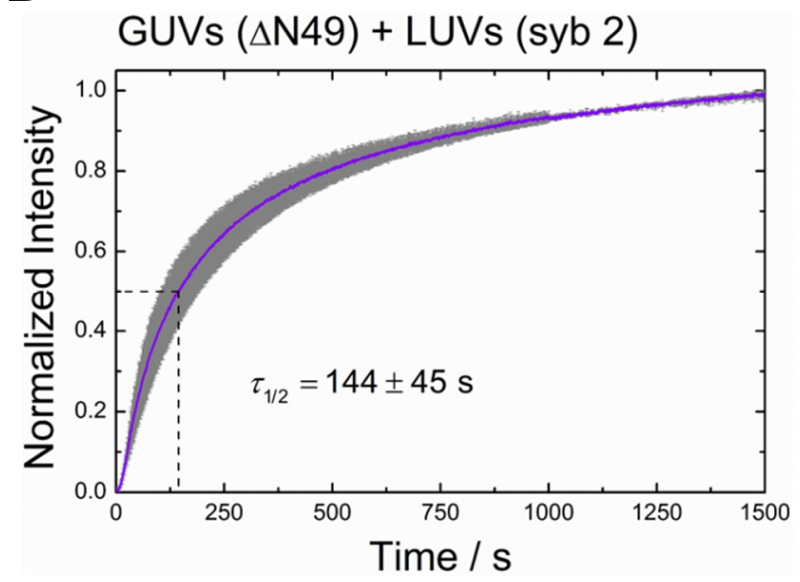

Figure 4-10 Normalized and time-resolved increase of the FRET-acceptor's (TxR-DPPE) fluorescence intensity in SNARE-mediated vesicle-vesicle bulk fusion experiments (violet line). Error bars are depicted in grey. (A) Average curve of 7 bulk fusion experiments of proteo-SUVs $(\Delta N 49)$ with proteo-SUVs (syb 2 ) leading to a value for $\tau_{1 / 2}$ of $114 \mathrm{~s}$. (B) Average curve of 6 distinct bulk fusion experiments of proteo-GUVs $(\Delta \mathrm{N} 49)$ with proteo-LUVs (syb 2) leading to a value for $\tau_{1 / 2}$ of $144 \mathrm{~s}$. 
After verification of functional reconstitution of the SNAREs into GUVs and LUVs, the $\triangle N 49$ doped GUVs were used for PSM preparation to investigate SNARE-mediated fusion on a single vesicle level. In 25 recorded time series on different PSM patches of 4 individual SNARE reconstitutions, 677 proteo-LUVs were monitored that docked to PSMs with a PI(4,5) $\mathrm{P}_{2}$ content of $5 \mathrm{~mol} \%$. By classifying the detected events separately in each of the 25 time series, a mean fusion efficiency of $83 \pm 3 \%$ was observed. Consequently, $17 \pm 2 \%$ of the vesicles that docked to the PSM were stalled in the docking state and did not proceed to fusion within the time window of observation. Among the $83 \%$ of the proteoliposomes that fused with the PSM, in average $51 \pm 4 \%$ proceeded to full fusion, whereas $49 \pm 4 \%$ remained in an intermediate fusion state. The resulting statistics of this analysis are compiled in a bar diagram in Figure 4-11.

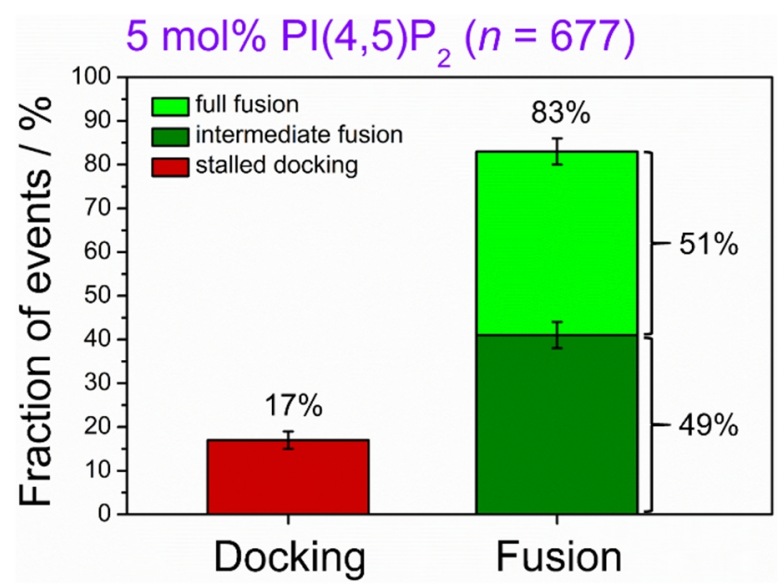

Figure 4-11 Statistical analysis of the fusion behavior of 677 detected proteo-LUVs (syb 2) docked to PSMs $(\Delta \mathrm{N} 49)$ with a PI $(4,5) \mathrm{P}_{2}$ content of $5 \mathrm{~mol} \%$. The underlying data was extracted from 25 recorded time series on different PSM patches of 4 individual SNARE reconstitutions. The depicted values for the fraction of events represent the mean percentage obtained by a separate evaluation of the fusion states in each recorded time series resulting in a fusion efficiency of $83 \pm 3 \%$. The standard deviation from the resulting mean percentage of each fusion state is depicted as error bar (black).

In case of a PI(4,5) $\mathrm{P}_{2}$ content of $5 \mathrm{~mol} \%$ in the PSMs, lifetimes of the docking state of 456 detected proteo-LUVs that fused with the PSM were determined. Fitting of Equation (4-1) to the obtained histogram of $N\left(\tau_{\text {docking }}\right)$ resulted in a value for $\tau_{\text {docking }}^{\text {max }} 33 \pm 2 \mathrm{~s}$ and a FWHM of $42 \mathrm{~s}$ (Figure 4-12A). From the intensity traces of 248 single vesicle fusion events, the lifetimes of the intermediate fusion state $\tau_{\text {intermediate }}$ were extracted. A histogram of the binned numbers of $\tau_{\text {intermediate }}$ is shown in Figure 4-12B. By fitting a mono exponential decay (Equation (4-3)) to the data, a rate constant $k$ for the decay of the intermediate fusion state of $0.248 \pm 0.014 \mathrm{~s}^{-1}$ and an average lifetime $\bar{\tau}_{\text {intermediate }}$ of $4.0 \pm 0.2 \mathrm{~s}$ were obtained. 
A

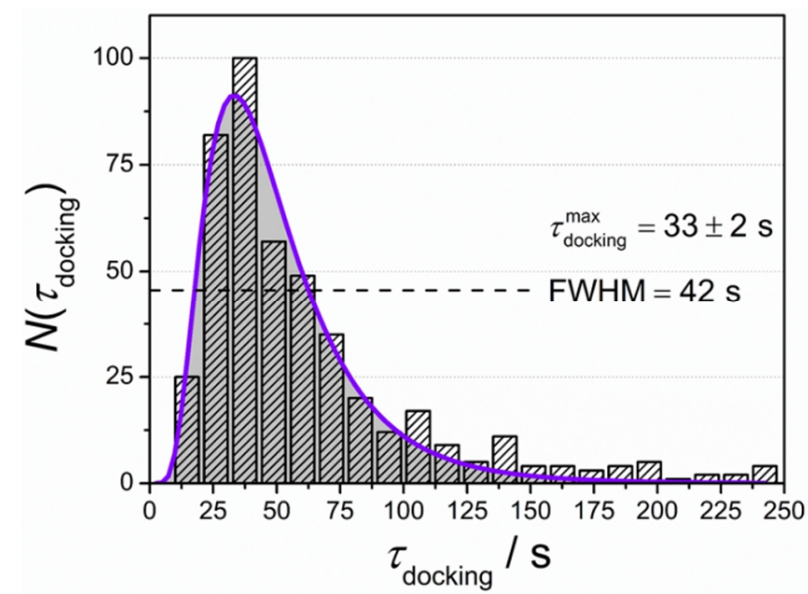

B

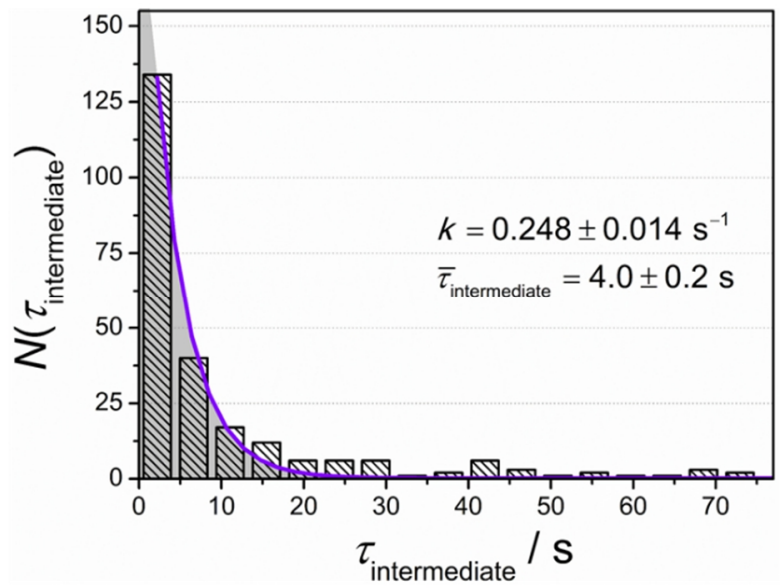

Figure 4-12 (A) Histogram of lifetimes of the docking state $\tau_{\text {docking }}$ of 456 proteo-LUVs on PSMs with a $\mathrm{PI}(4,5) \mathrm{P}_{2}$ content of $5 \mathrm{~mol} \%$. Fitting of a log-normal distribution (Equation (4-1)) to the data resulted in a maximum value for the docking lifetime of $33 \mathrm{~s}$ and a FWHM of $42 \mathrm{~s}$. (B) Histogram of lifetimes of the intermediate fusion state $\tau_{\text {intermediate }}$ of a total number of 248 detected events on PSMs doped with $5 \mathrm{~mol} \%$ $\mathrm{PI}(4,5) \mathrm{P}_{2}$. Fitting of a mono exponential decay (Equation (4-3)) to the data resulted in a rate constant $\mathrm{k}$ for the decay of the intermediate fusion state of $0.248 \mathrm{~s}^{-1}$ and an average lifetime $\bar{\tau}_{\text {intermediate }}$ of $4.0 \mathrm{~s}$.

\subsubsection{Comparison of SNARE-mediated single vesicle fusion on pore- spanning membranes with different $\mathrm{PI}(4,5) \mathrm{P}_{2}$ contents}

One major goal of this work was to obtain new insights into the impact of the phosphoinositide $\mathrm{PI}(4,5) \mathrm{P}_{2}$ on SNARE-mediated single vesicle fusion in vitro, as it was observed that $\mathrm{PI}(4,5) \mathrm{P}_{2}$ is the dominant inner leaflet lipid in PC12 cells at sites of fusion. ${ }^{[51]}$ For this purpose, the $\mathrm{PI}(4,5) \mathrm{P}_{2}$ content in PSMs containing the t-SNARE complex $\Delta \mathrm{N} 49$ was gradually increased from $1 \mathrm{~mol} \%$ to $2 \mathrm{~mol} \%$ to $5 \mathrm{~mol} \%$. The fusion counterpart, syb 2 doped LUVs, however, was always prepared with the same lipid mixture and the same amount of protein. An overview on the statistical analysis of the fusion states that were monitored for docked proteo-LUVs on PSMs with varying $\mathrm{PI}(4,5) \mathrm{P}_{2}$ contents is shown in a bar diagram in Figure 4-13. The detected events, to that end, were categorized into the fusion states docking, intermediate fusion or full fusion depending on the progress until the end of the time window of observation (cf. Figure 4-3). Concerning the fusion efficiency for proteo-LUVs docked to PSM with a PI(4,5) $\mathrm{P}_{2}$ content of $1 \mathrm{~mol} \%(79 \pm 4 \%)$ or $5 \mathrm{~mol} \%(83 \pm 3 \%)$ no significant difference was observed. For proteoliposomes docked to PSMs with a $\mathrm{PI}(4,5) \mathrm{P}_{2}$ content of $2 \mathrm{~mol} \%$, however, a significantly higher fusion efficiency of $92 \pm 4 \%$ was observed. With regard on the vesicles that fused with the PSM, the relation between intermediate fusion and full fusion was determined to be the same ( 1:1) on PSMs with different amounts of PI(4,5) $\mathrm{P}_{2}$. 


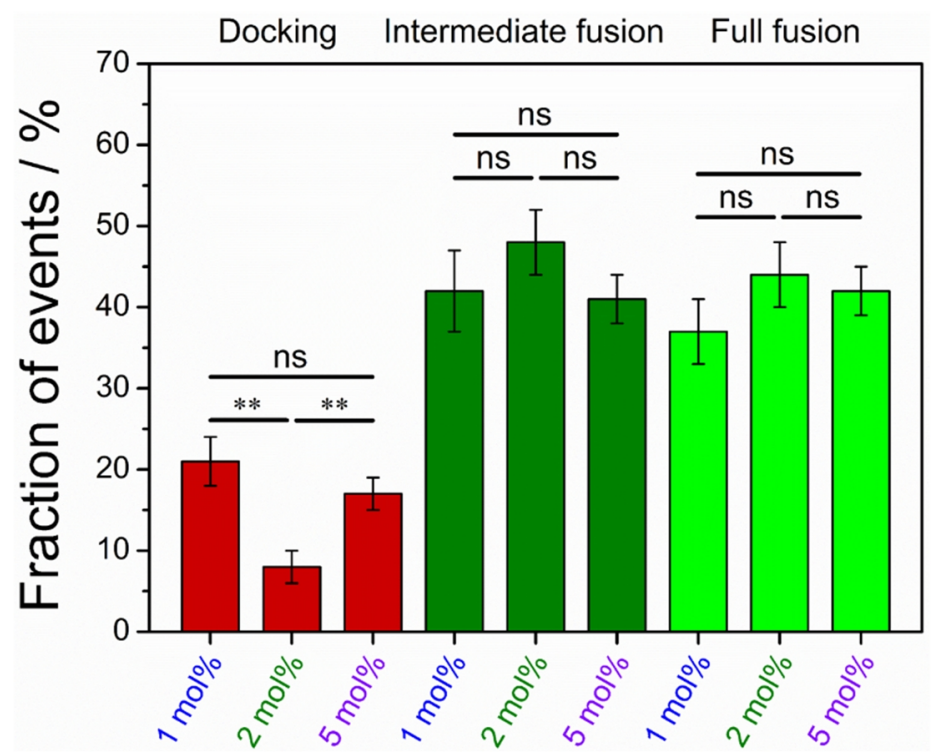

Figure 4-13 Comparison of the mean fractions of fusion states monitored for single vesicle events $n$ on PSMs with different amounts of PI $(4,5) \mathrm{P}_{2}(1 \mathrm{~mol} \%(\mathrm{n}=537), 2 \mathrm{~mol} \%(\mathrm{n}=994)$ and $5 \mathrm{~mol} \%(\mathrm{n}=677))$. Statistical independent two-sample t-test: $* *$ : $<<0.01$, ns: not significant.

From a more detailed analysis of the lifetime of the docking state $\tau_{\text {docking }}$ of proteo-LUVs on PSMs with different amounts of $\mathrm{PI}(4,5) \mathrm{P}_{2}$, it was hoped to gain new findings concerning the influence of $\mathrm{PI}(4,5) \mathrm{P}_{2}$ on the kinetics of SNARE-mediated single vesicle fusion in vitro. To this end, docking lifetimes (cf. Figure 4-3) were extracted from the intensity traces of single vesicle fusion events monitored on PSMs with different amounts of $\mathrm{PI}(4,5) \mathrm{P}_{2}$. The determined values for $\tau_{\text {docking }}$ were binned according to the FREEDMAN-DIACONIS rule ${ }^{[121]}$ and plotted in a histogram. This was performed separately for each $\mathrm{PI}(4,5) \mathrm{P}_{2}$ content $(1,2$ and $5 \mathrm{~mol} \%)$. The maxima of the resulting docking time distributions were determined by fitting a log-normal distribution (Equation (4-1)) to the data and calculating the mode of the obtained fit function

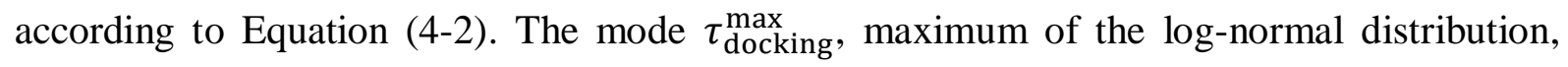
describes the value for the docking lifetime that is most likely to be observed for the proteoLUV docked to PSMs with a certain $\mathrm{PI}(4,5) \mathrm{P}_{2}$ content. To quantify the broadness of the resulting docking time distribution the full width at half maximum (FWHM) of the log-normal fit function was calculated. The resulting value for $\tau_{\text {docking }}^{\max }$ and the corresponding FWHM for each $\mathrm{PI}(4,5) \mathrm{P}_{2}$ content is listed in Table 4-5. 
Table 4-5 Values for $\tau_{\text {docking }}^{\max }$ and the corresponding FWHM of the log-normal distributions (Equation (4-1)) fitted to the histograms of determined docking lifetimes of proteo-LUVs on PSMs with different $\mathrm{PI}(4,5) \mathrm{P}_{2}$ contents.

\begin{tabular}{cccc}
\hline & 1 mol\% PI(4,5)P & 2 mol\% PI(4,5) $\mathbf{P}_{\mathbf{2}}$ & $\mathbf{5}$ mol\% PI(4,5) $\mathbf{P}_{\mathbf{2}}$ \\
\hline $\boldsymbol{\tau}_{\text {docking }}^{\text {max }}$ & $38 \pm 3 \mathrm{~s}$ & $44 \pm 1 \mathrm{~s}$ & $33 \pm 2 \mathrm{~s}$ \\
FWHM & $47 \mathrm{~s}$ & $55 \mathrm{~s}$ & $42 \mathrm{~s}$ \\
\hline
\end{tabular}

In case of docking lifetimes, again only a minor difference between 1 and $5 \mathrm{~mol} \% \mathrm{PI}(4,5) \mathrm{P}_{2}$ was observed. A more significant difference in the distribution of docking lifetimes was determined for proteo-LUVs on PSMs with a $\mathrm{PI}(4,5) \mathrm{P}_{2}$ content of $2 \mathrm{~mol} \%$ in comparison to 1 and $5 \mathrm{~mol} \%$. Here, the maximum was shifted by a factor of $\sim 1.2$ to a longer docking lifetime and the distribution was found to be broader. In the histograms of the docking lifetimes for the different $\mathrm{PI}(4,5) \mathrm{P}_{2}$ contents (Figure 4-14) a clear in- and decrease in the frequency of events can be observed. Such a course is typical for processes which involve multiple steps, whereas mechanisms involving only a single step would result in a mono exponential decay. ${ }^{[122]}$ As many biochemical processes consist of more than one step, VAN OIJEN and coworkers introduced a general kinetic model (Equation (4-4)) which describes the probability of a transition from state $A$ to state $B$ at a time $\tau$

$$
p_{A \rightarrow B}(\tau)=\frac{k^{N} \cdot \tau^{N-1}}{\Gamma(N)} \cdot \exp (-k \cdot \tau)
$$

where $N$ is the number of hidden transition steps, $k$ the rate constant and $\Gamma(N)$ the gamma function. This model assumes a uniform rate constant $k$ for all intermediate steps, what is a reasonable assumption, as only the slowest step in the entire reaction significantly contributes to $p_{A \rightarrow B}(\tau) .{ }^{[122]}$ This model was fitted to the docking lifetime distributions of proteo-LUVs on PSMs with different $\mathrm{PI}(4,5) \mathrm{P}_{2}$ contents to get a hint on the number of hidden reaction steps that are passed during the transition from docking to fusion. Fitting of the kinetic model to the data was conducted assuming an integer number of steps $N=2-5$. To estimate the quality of the fit for each $N$, the corresponding reduced $\chi^{2}$ values were compared. One finds the lowest reduced $\chi^{2}$ values for $N=4$, resulting in rate constants for the different amounts of $\mathrm{PI}(4,5) \mathrm{P}_{2}$ of $k_{1 \mathrm{~mol} \%}=0.075 \pm 0.003 \mathrm{~s}^{-1}, k_{2 \mathrm{~mol} \%}=0.065 \pm 0.001 \mathrm{~s}^{-1}$ and $k_{5 \mathrm{~mol} \%}=0.084 \pm 0.003 \mathrm{~s}^{-1}$ (cf. Figure 4-14D). 

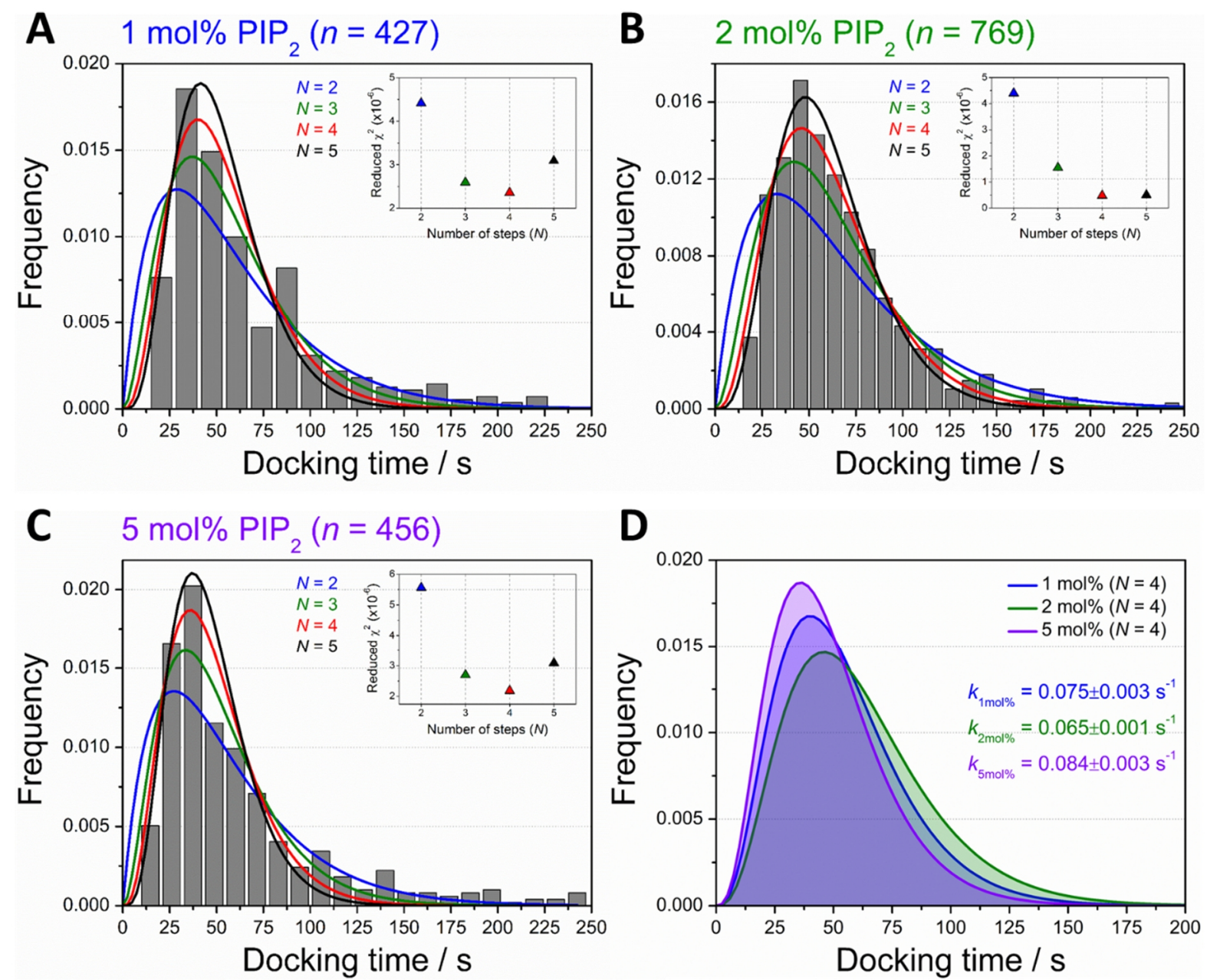

Figure 4-14 (A)-(C) Histograms of docking lifetimes of proteo-LUVs docked to PSMs with different amounts of $\mathrm{PI}(4,5) \mathrm{P}_{2}$. Equation (4-4) with $\mathrm{N}=2-5$ was fitted to the data to determine the number of hidden steps that are passed during the transition from docking to fusion. The insets depict the reduced $\chi^{2}$ values derived from the different fits indicating $\mathrm{N}=4$ as the number of steps where the data is modeled best. (D) Plot of the resulting fit functions (Equation (4-4)) with $\mathrm{N}=4$ for each $\mathrm{PI}(4,5) \mathrm{P}_{2}$ content leading to the rate constants $\mathrm{k}_{1 \mathrm{~mol} \%}=0.075 \pm 0.003 \mathrm{~s}^{-1}, \mathrm{k}_{2 \mathrm{~mol} \%}=0.065 \pm 0.001 \mathrm{~s}^{-1}$ and $\mathrm{k}_{5 \mathrm{~mol} \%}=0.084 \pm 0.003 \mathrm{~s}^{-1}$.

Another observable that was extracted from the intensity traces of proteo-LUVs that fused with PSMs containing different amounts of $\mathrm{PI}(4,5) \mathrm{P}_{2}$ was the lifetime of the intermediate fusion state defined as the time between the onset of lipid mixing and the start of decay of the $\Omega$-shaped post-fusion structure of the LUV into the PSM (cf. Figure 4-3). In contrast to the distributions of the docking lifetimes, the distributions of intermediate fusion lifetimes (cf. Figure 4-6B, Figure 4-9B and Figure 4-12B) were characterized by a single decrease suggesting a simple one step mechanism of this process. By fitting a mono exponential decay (Equation (4-3)) to the data, the rate constant $k$ for the decay of the intermediate fusion state and the corresponding average lifetime $\bar{\tau}_{\text {intermediate }}$ as the reciprocal of $k$ could be obtained. The determined values for $k$ and $\bar{\tau}_{\text {intermediate }}$ of the intermediate fusion state of proteo-LUVs fusing with PSMs containing different amounts of $\mathrm{PI}(4,5) \mathrm{P}_{2}$ are listed in Table 4-6. 
Table 4-6 Values for the rate constant $\mathrm{k}$ and the corresponding average lifetime $\bar{\tau}_{\text {intermediate }}$ of the intermediate fusion state of proteo-LUVs fusing with PSMs containing different amounts of $\mathrm{PI}(4,5) \mathrm{P}_{2}$.

\begin{tabular}{|c|c|c|c|}
\hline & $1 \mathrm{~mol}^{2} \mathrm{PI}(4,5) \mathrm{P}_{2}$ & $2 \mathrm{~mol} \% \mathrm{PI}(4,5) \mathrm{P}_{2}$ & $5 \mathrm{~mol} \% \mathrm{PI}(4,5) \mathrm{P}_{2}$ \\
\hline $\boldsymbol{k}$ & $0.162 \pm 0.005 \mathrm{~s}^{-1}$ & $0.147 \pm 0.018 \mathrm{~s}^{-1}$ & $0.248 \pm 0.014 \mathrm{~s}^{-1}$ \\
\hline$\overline{\boldsymbol{\tau}}_{\text {intermediate }}$ & $6.2 \pm 0.2 \mathrm{~s}$ & $6.8 \pm 0.8 \mathrm{~s}$ & $4.0 \pm 0.2 \mathrm{~s}$ \\
\hline
\end{tabular}

With regard on the average lifetime of the intermediate fusion state of proteo-LUVs on PSMs containing 1 or $2 \mathrm{~mol} \% \mathrm{PI}(4,5) \mathrm{P}_{2}$, no significant difference was observed, whereas the average lifetime of the post-fusion structure was determined to be shorter on PSMs with 5 mol\% $\mathrm{PI}(4,5) \mathrm{P}_{2}$ by a factor of $\sim 1.6$.

\subsection{Impact of syt-1 on SNARE-mediated fusion}

The key step of neuronal signal transmission in a chemical synapse is the $\mathrm{Ca}^{2+}$-dependent synchronous secretion of neurotransmitter-loaded synaptic vesicles on a sub-millisecond time scale. In order to ensure this highly regulated synchronous process upon $\mathrm{Ca}^{2+}$-influx, a sensitive $\mathrm{Ca}^{2+}$-sensor is required. Syt-1 was determined to be a main candidate for a $\mathrm{Ca}^{2+}$-sensor in neuronal exocytosis but the exact mechanism, how syt-1 triggers $\mathrm{Ca}^{2+}$-evoked neurotransmitter release, still remains unclear. For this reason, investigation of the impact of syt-1 on SNAREmediated membrane fusion under well-defined conditions in vitro is of great interest to get a better understanding of syt-1's mode of action.

\subsubsection{Isolation and purification of syt-1}

Recombinant expression of syt-1 in a suited host strain as well as the subsequent extraction and purification of syt-1 was a prerequisite to analyze its impact on SNARE-mediated membrane fusion in vitro. To this end, transformed E. coli (BL21-CodonPlus (DE3)-RIPL) cells, carrying a pET-28a(+) vector with the inserted DNA sequence encoding for syt-1 (rattus norvegicus, aa 1-421), were utilized as a host strain for expression. After cell lysis, extraction of syt-t, carrying a His6-tag, was accomplished by $\mathrm{Ni}^{2+}$-affinity chromatography, followed by a thrombin catalyzed removal of the His 6 -tag and final purification by ion exchange chromatography (IEC). Successful expression of syt-1 and segregation from other contaminating proteins after cell lysis was verified by SDS-PAGE analysis. SDS-PAGE gels after cell lysis and $\mathrm{Ni}^{2+}$-affinity 
chromatography as well as after His6-tag cleavage of an exemplary syt-1 isolation are shown in Figure 4-15.
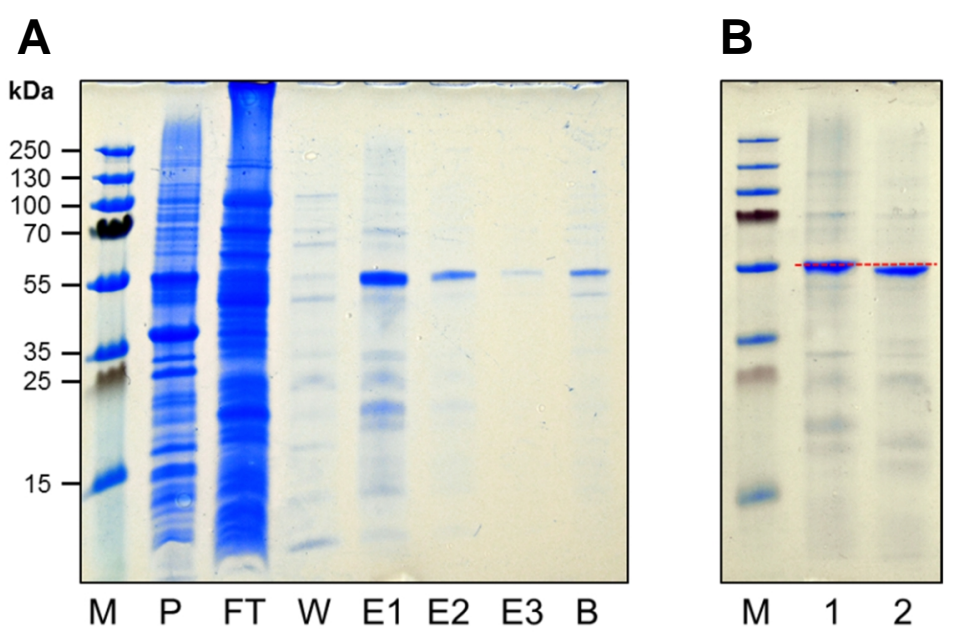

Figure 4-15 SDS-PAGE analysis of an exemplary syt-1 ( $\mathrm{M}=47.4 \mathrm{kDa})$ isolation. (A) Gel for the control of cell lysis, expression and $\mathrm{Ni}^{2+}$-affinity chromatography (M: marker, P: pellet, FT: flow through, W: wash, E1-E2: elution fractions, B: beads). (B) Gel for the control of His6-tag cleavage (M: Marker, 1: before cleavage (pooled elution fractions before thrombin addition), 2: after cleavage (pooled elution fractions after thrombin addition and dialysis overnight)). His6-tag removal leads to a minimal shift in molecular weight of syt-1 from $49.6 \mathrm{kDa}$ (+His6-tag) to $47.4 \mathrm{kDa}$ (-His6-tag) (red bar as a guide for the eye).

After the confirmation of successful expression and removal of the His 6 -tag by SDS-PAGE, syt-1 was further purified using the strong anion exchange column MonoQ 5/50 (GE Healthcare, Little Chalfont, UK). The resulting ÄKTA chromatogram as well as the SDSPAGE analysis of the elution fractions of interest are depicted in Figure 4-16.

A

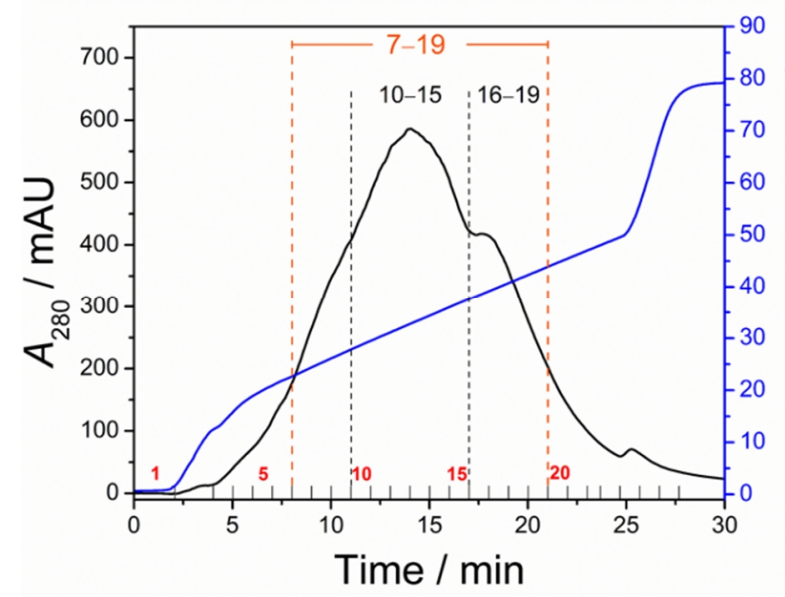

B

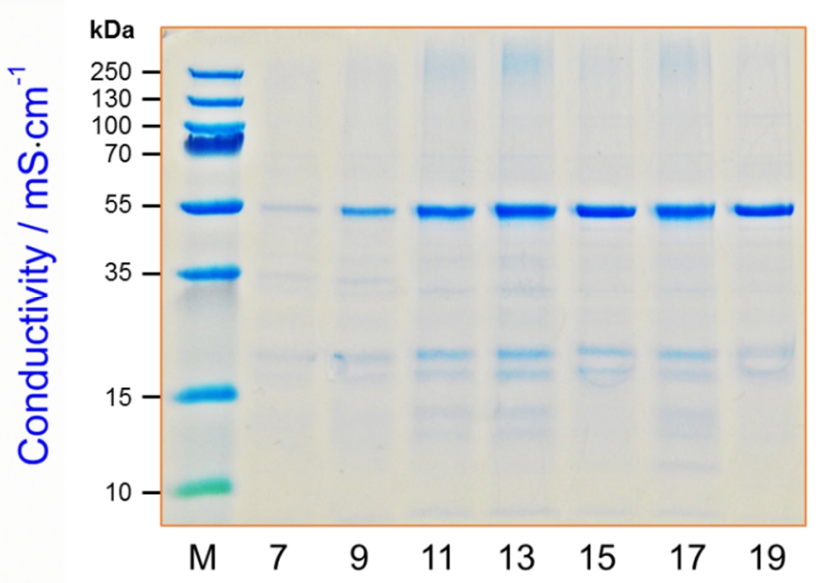

Figure 4-16 (A) Ion exchange chromatogram obtained after purification of syt- 1 on a MonoQ 5 / 50 column. Syt-1 $(\mathrm{M}=47.4 \mathrm{kDa})$ elutes in a conductivity range between $22.56 \mathrm{mS} / \mathrm{cm}$ and $43.87 \mathrm{mS} / \mathrm{cm}$ (fractions 719). (B) SDS-PAGE analysis of the elution fractions 7-19 showing a dominant band at $\sim 50 \mathrm{kDa}$.

The dominant band in the elution fractions $9-19$ at $\sim 50 \mathrm{kDa}$ was assigned to the target protein syt-1. It elutes in a conductivity range between $22.56-43.87 \mathrm{mS} / \mathrm{cm}$ with a maximum value 
for $A_{280}$ at $32.61 \mathrm{mS} / \mathrm{cm}$ (Figure 4-16A). As a shoulder was observed in the ÄKTA chromatogram (fractions 16-19), fractions 10-15 and 16-19 were combined separately. The total protein concentration for both pooled fractions was determined by UV-vis spectroscopy using the NanoDrop 2000c (Thermo Fischer Scientific, Waltham, MA, USA). Estimating a calculated extinction coefficient for the sequence of syt-1 (7.1.4) of $\varepsilon=38760 \mathrm{M}^{-1} \cdot \mathrm{cm}^{-1}$, syt-1 concentrations for $\operatorname{pool}_{1}(10-15)$ and $\operatorname{pool}_{2}(16-19) \quad$ of $\quad c_{1}(\mathrm{syt}-1)=26.4 \mu \mathrm{M}$ and $c_{2}$ (syt-1) $=17.1 \mu \mathrm{M}$ were determined. Taking the volumes of the two pools into account, $V\left(\right.$ pool $\left._{1}\right)=6 \mathrm{~mL}$ and $V\left(\right.$ pool $\left._{2}\right)=4 \mathrm{~mL}$, a total of $10.75 \mathrm{mg}$ syt-1 was isolated from $2.5 \mathrm{~L}$ main culture. As the occurrence of the shoulder in the ÄKTA chromatogram was the reason for separated pooling, both pools were further analyzed in more detail to check for a difference in contaminations by SDS-PAGE analysis. For this purpose, three different amounts of protein $(1.9 \mu \mathrm{g}, 4.4 \mu \mathrm{g}$ and $6.3 \mu \mathrm{g}$ ) of each pool were loaded onto the SDS gel and submitted to electrophoresis. As no significant difference in contamination was observed (Figure 4-17A), both pooled fractions were combined and further concentrated using a vivaspin 6 concentrator tube (Sartorius, Göttingen, Germany) with a MWCO of $5 \mathrm{kDa}$. Centrifugal concentration of the combined pools (fractions 10-19) resulted in a final concentration of $c$ (syt-1) $=63.3 \mu \mathrm{M}$. Again SDS-PAGE analysis of this concentrated, final syt-1 solution was performed with two different protein amounts as shown in Figure 4-17B.

A

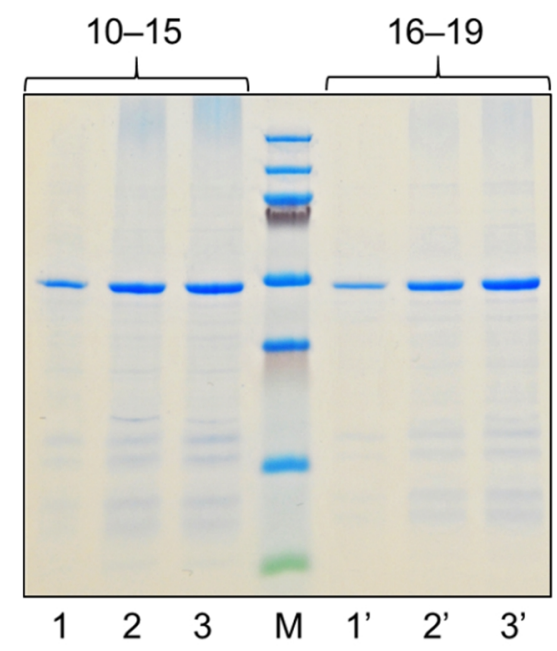

B

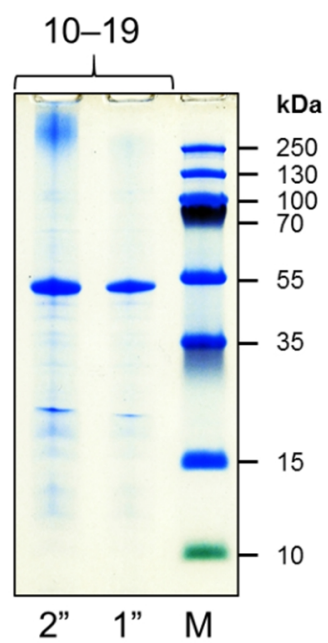

Figure 4-17 (A) SDS-PAGE analysis of the pooled ÄKTA fractions 10-15 and 16-19. Protein amounts of $1.9 \mu \mathrm{g}\left(1,1^{\prime}\right), 4.4 \mu \mathrm{g}\left(2,2^{\prime}\right)$ and $6.3 \mu \mathrm{g}\left(3,3^{\prime}\right)$ were loaded onto the gel (M: Marker). (B) SDS-PAGE analysis of the combined pools (ÄKTA fractions 10-19) after increasing the syt-1 concentration to $63.3 \mu \mathrm{M}$ using a vivaspin 6 centrifugal concentrator tube (MWCO $=5 \mathrm{kDa})$. Protein amounts of $3.5 \mu \mathrm{g}(1 ")$ and $7.0 \mu \mathrm{g}\left(2^{\prime \prime}\right)$ were analyzed (M: Marker).

The isolated syt- 1 of high purity was divided into aliquots $(V=11 \mu \mathrm{L})$ and stored at $-80{ }^{\circ} \mathrm{C}$. 


\subsubsection{Reconstitution of syt-1 into liposomes and analysis of the $\mathrm{Ca}^{2+}$ independent interaction with $\mathrm{PI}(4,5) \mathrm{P}_{2}$}

With the purified syt-1 in hands, the next step was to reconstitute it into artificial liposomes and to investigate its $\mathrm{Ca}^{2+}$-independent binding affinity to solid supported membranes (SSMs) doped with the polyanionic phospholipid $\mathrm{PI}(4,5) \mathrm{P}_{2}$. To this end, syt-1 was integrated into small unilamellar vesicles (SUVs) in a protein to lipid ratio of $\mathrm{p} / 1=1: 1000$ using the standard comicellization protocol according to HERNANDEZ (cf. Chapter 3.2.5). Successful reconstitution of syt-1 into SUVs was verified by density centrifugation in a Nycodenz gradient, by which unreconstituted, free protein is segregated from that one, which was integrated into the liposomes (cf. Chapter 3.2.10). After ultracentrifugation, fractions were carefully taken from top down and analyzed by SDS-PAGE analysis. A picture of the samples taken after ultracentrifugation as well as the resulting SDS gel is shown in Figure 4-18.
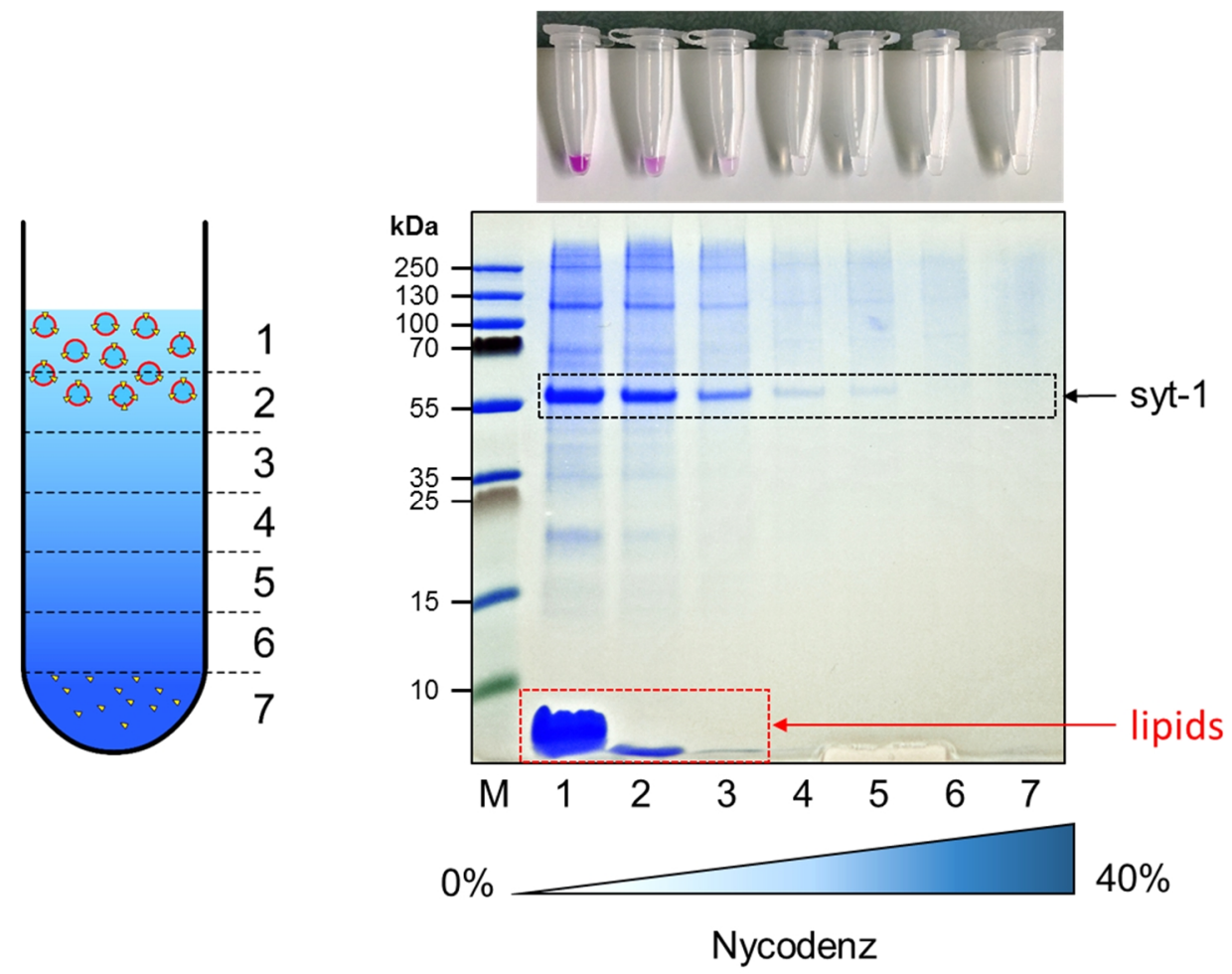

Figure 4-18 Nycodenz assay to analyse the reconstitution of syt-1 $(\mathrm{p} / \mathrm{l}=1: 1000)$ into SUVs by the comicellization method. Fractions (1-7) were taken from top down after ultracentrifugation of the syt-1/ SUVsuspension in a Nycodenz gradient (0-40 \% (w/v)) to segregate proteoliposomes from unreconstituted protein as schematically depicted on the left. SDS-PAGE analysis of the taken samples (1-7) verified successful reconstitution of syt-1 into SUVs by showing the most prominent bands in the fractions of lowest Nycodenz density and highest lipid content (fractions 1-3).

The strong colocalization of lipid material and syt-1 in the fractions of lowest Nycodenz density, as determined by SDS-PAGE analysis, verified successful reconstitution of syt-1 into SUVs. To ensure that syt-1 remains functional after reconstitution into SUVs, RIfS 
measurements were carried out to analyze syt-1-mediated binding of proteo-SUVs to SSMs doped with the polyanionic receptor lipid $\mathrm{PI}(4,5) \mathrm{P}_{2}$. Figure 4-19A shows the course of an exemplary RIfS measurement, monitoring the binding of syt-1 containing SUVs to a SSM with a PI(4,5) $\mathrm{P}_{2}$ content of $5 \mathrm{~mol} \%$. The experiment was started with the addition of SUVs composed of DOPC/POPE/POPS/Chol/PI(4,5) $\mathrm{P}_{2}(45: 20: 10: 20: 5,0.2 \mathrm{mg} / \mathrm{mL})$ in citrate buffer $(20 \mathrm{mM}$ Na-citrate, $50 \mathrm{mM} \mathrm{KCl}, 0.1 \mathrm{mM}$ EDTA, $0.1 \mathrm{mM} \mathrm{NaN}_{3}, \mathrm{pH} 4.8$ ) to a silicon wafer with a hydrophilic $\mathrm{SiO}_{2}$-layer of $5 \mu \mathrm{m}$. Spreading of the SUVs on top of the hydrophilic surface resulted in the formation of a $\mathrm{PI}(4,5) \mathrm{P}_{2}$ containing $\mathrm{SSM}$, leading to an increase of $\triangle O T$ to $7.3 \mathrm{~nm}$ (physical thickness $d=4.9 \mathrm{~nm}$ ) and was finished after $\sim 30 \mathrm{~min}$. The citrate buffer with a $\mathrm{pH}$ of 4.8 was needed to facilitate the spreading process in case of $\mathrm{PI}(4,5) \mathrm{P}_{2}$ concentrations of $5 \mathrm{~mol} \%$ to reduce the negative surface charge of the SUVs by protonating the $\mathrm{PI}(4,5) \mathrm{P}_{2}$ headgroup. ${ }^{[123]}$ For this reason a buffer exchange was carried out after the completed formation of the SSM by rinsing the measuring chamber in an open system with SNARE buffer for at least $10 \mathrm{~min}$ (flowrate: $0.9 \mathrm{~mL} / \mathrm{min}$ ). Rinsing was followed by the addition of syt-1 containing SUVs, leading to a second increase of $\triangle O T$. The difference between $\triangle O T$ after SSM formation and the maximum of $\triangle O T$ after proteo-SUV binding is referred to as $\Delta(\triangle O T)_{\max }$.

A

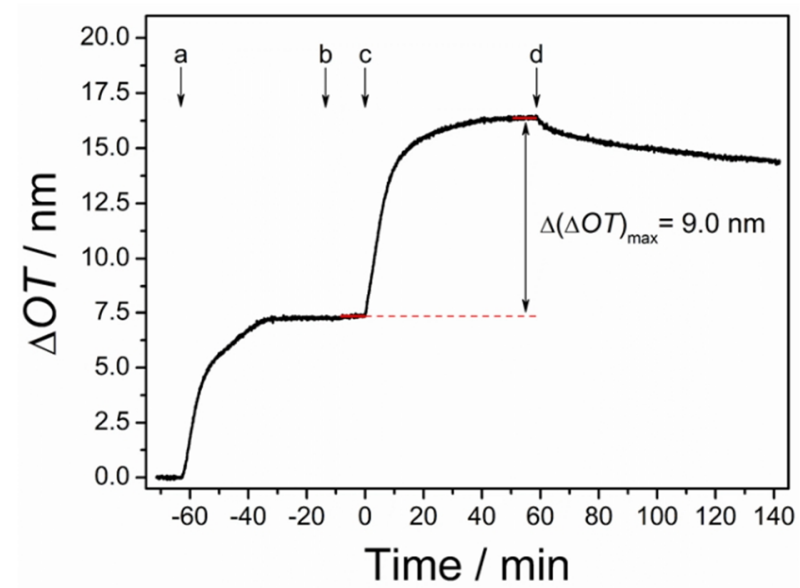

B

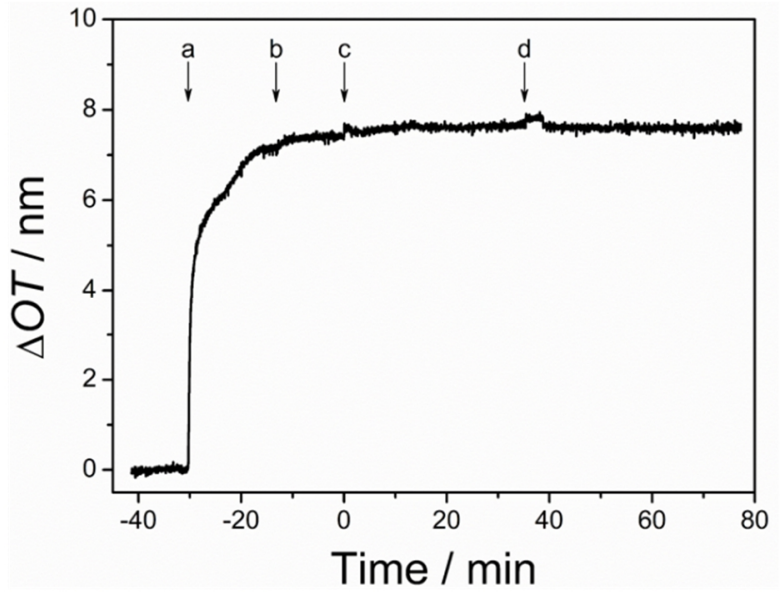

Figure 4-19 (A) Representative time-resolved course of $\Delta$ OT during a RIfS measurement to analyze the binding of syt-1 containing SUVs to SSMs doped with the receptor lipid PI $(4,5) \mathrm{P}_{2}$. In the depicted example the PI $(4,5) \mathrm{P}_{2}$ content of the SSM was adjusted to $5 \mathrm{~mol} \%$ (a: addition of SUVs in citrate buffer containing 5 mol\% PI(4,5) $\mathrm{P}_{2}$ to form a SSM; b: rinsing with SNARE buffer; c: addition of syt-1 containing SUVs; d: rinsing with SNARE buffer). (B) Corresponding control RIfS measurement on a SSM with a PI $(4,5) \mathrm{P}_{2}$ content of $5 \mathrm{~mol} \%$ (c: addition of SUVs without reconstituted syt-1; a,b,d: same as in (A)).

The course of the corresponding control experiment, in which vesicles without syt-1 were added to a SSM doped with 5 mol\% $\mathrm{PI}(4,5) \mathrm{P}_{2}$, is shown in Figure 4-19B. Here, no second increase in $\triangle O T$ was observed, ruling out unspecific vesicle adhesion to the $\mathrm{PI}(4,5) \mathrm{P}_{2}$ containing SSMs. Also for the case of SSMs lacking the receptor lipid $\mathrm{PI}(4,5) \mathrm{P}_{2}$, no increase in $\triangle O T$ was observed 
after the addition of syt-1 containing SUVs $(n=3)$. The binding of SUVs with reconstituted syt-1 to SSMs with a PI(4,5) $\mathrm{P}_{2}$ content of 1,2 and 5 mol\% was analyzed by means of RIfS ( $n=7$ for each $\mathrm{PI}(4,5) \mathrm{P}_{2}$ content). The mean of the obtained $\Delta(\Delta O T)_{\max }$-values with the corresponding standard deviations for each $\mathrm{PI}(4,5) \mathrm{P}_{2}$ content is depicted in Figure 4-20.

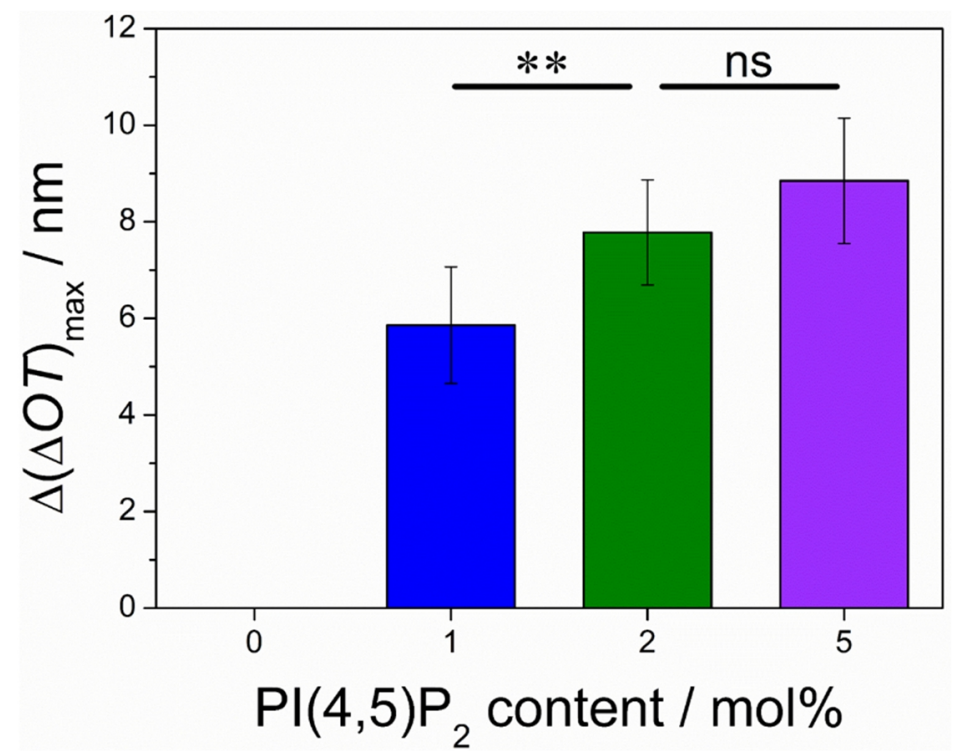

Figure 4-20 Bar diagram of the mean values of $\Delta(\Delta \mathrm{OT})_{\max }$ obtained by binding of SUVs with reconstituted syt-1 $(\mathrm{p} / \mathrm{l}=1: 1000)$ to SSMs with different $\mathrm{PI}(4,5) \mathrm{P}_{2}$ contents $\left(\mathrm{n}=7\right.$ for each $\mathrm{PI}(4,5) \mathrm{P}_{2}$ content). In case of SSMs with a composition of DOPC/ POPE/ POPS/ Chol (50:20:10:20), no binding of syt-1 doped SUVs to SSMs was observed $(\mathrm{n}=3)$. Statistical independent two-sample t-test: **: $\mathrm{p}<0.01$, ns: not significant.

The results from the Nycodenz assay and the RIfS measurements confirmed successful reconstitution of syt-1 into SUVs and verified the specific interaction between syt-1 and $\mathrm{PI}(4,5) \mathrm{P}_{2}$.

To prepare syt-1 containing LUVs, the proteo-SUV suspension was transferred to a $5 \mathrm{~mL}$ round bottom flask and dried overnight at $4{ }^{\circ} \mathrm{C}$ in a desiccator over saturated sodium chloride solution, followed by rehydration in SNARE buffer and extrusion through a polycarbonate membrane with pore sizes of $400 \mathrm{~nm}$ in diameter. With the obtained proteo-LUVs, RIfS measurements were performed to ensure the preserved functionality of syt-1 after these preparation steps. Unfortunately, no binding of the LUVs to $\mathrm{PI}(4,5) \mathrm{P}_{2}$ containing SSMs was monitored not even in the case of a $\mathrm{PI}(4,5) \mathrm{P}_{2}$ content of $5 \mathrm{~mol} \%$ in the SSM. For this purpose, another reconstitution protocol had to be developed to yield LUVs with functionally reconstituted syt-1. 


\subsubsection{Co-reconstitution of syt-1 and syb 2 into liposomes and analysis of single vesicle fusion events}

As the standard co-micellization method for the reconstitution of SNARE-proteins into LUVs included a drying process, this reconstitution protocol was not applicable for syt-1 which turned out to be more sensitive regarding denaturation and loss of functionality. Therefore, a milder method for the reconstitution of syt-1 into LUVs was required which circumvents these harsh conditions. The reconstitution protocol of choice, in this case, was the direct insertion method (cf. Chapter 3.2.7) in which preformed LUVs were destabilized by the mild detergent $n$-octyl- $\beta$-D-thioglucopyranoside ( $n$-OG) followed by the addition and insertion of syt- 1 . The subsequent removal of the detergent was achieved by overnight dialysis and two steps of size exclusion chromatography. Due to the fact that this reconstitution method excludes drying, it resulted in the functional incorporation of syt-1 into LUVs as confirmed by RIfS measurements. For LUVs doped with syt-1 by the direct insertion protocol, binding to SSMs with different amounts of $\mathrm{PI}(4,5) \mathrm{P}_{2}$ was observed. In this case, also the binding to SSMs without $\mathrm{PI}(4,5) \mathrm{P}_{2}$ (PC/PE/PS/Chol (50:20:10:20)) was monitored ( $n=3)$. However, the control experiment with LUVs prepared under the same conditions but without syt-1 did not show binding to SSMs with a $\mathrm{PI}(4,5) \mathrm{P}_{2}$ content of $5 \mathrm{~mol} \%(n=2)$. In Figure $4-21$ a histogram of the obtained means of $\Delta\left(\Delta O T_{\max }\right)$ for the binding of syt-1 containing LUVs to SSMs with $\mathrm{PI}(4,5) \mathrm{P}_{2}$ contents of $0 \mathrm{~mol} \%, 1 \mathrm{~mol} \%, 2 \mathrm{~mol} \%$ and $5 \mathrm{~mol} \%$ is shown.

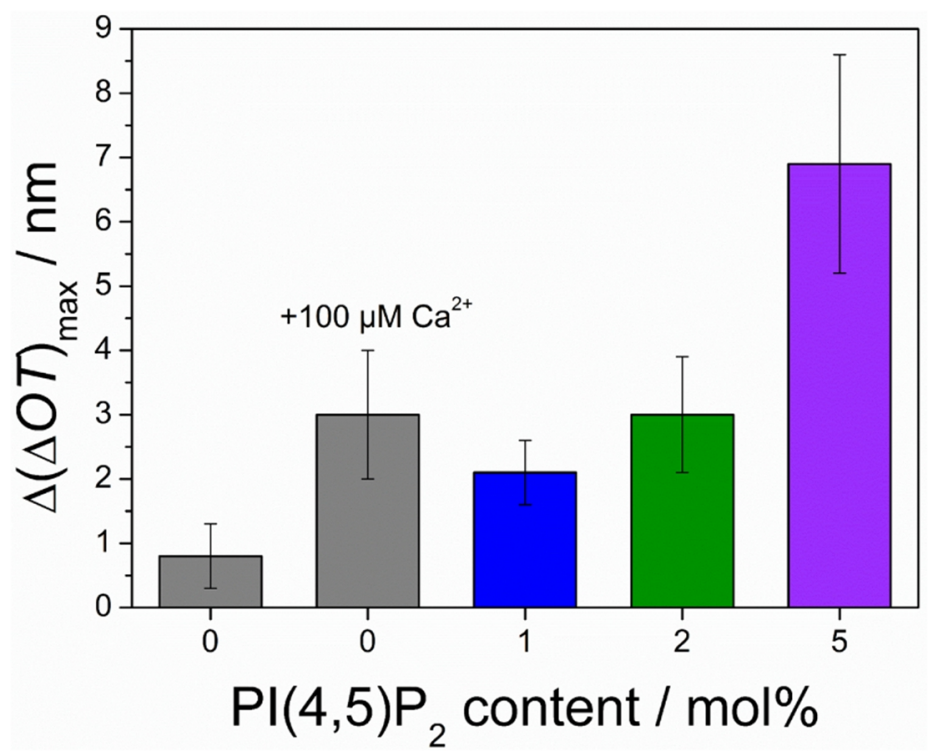

Figure 4-21 Bar diagram of the mean values of $\Delta(\Delta \mathrm{OT})_{\max }$ obtained by binding of syt-1 containing LUVs $(\mathrm{p} / \mathrm{l}=1: 1000)$ to SSMs with different $\mathrm{PI}(4,5) \mathrm{P}_{2}$ contents. For syt-1 reconstitution the direct insertion method was applied. In case of SSMs without PI(4,5) $\mathrm{P}_{2}$ (DOPC/ POPE/ POPS/ Chol (50:20:10:20)), additional measurements $(n=3)$ in the presence of $100 \mu \mathrm{M} \mathrm{Ca}^{2+}$ were conducted. All the other measurements with $\mathrm{PI}(4,5) \mathrm{P}_{2}$ contents $0 \mathrm{~mol} \%(\mathrm{n}=3), 1 \mathrm{~mol} \%(\mathrm{n}=3), 2 \mathrm{~mol} \%(\mathrm{n}=5)$ and $5 \mathrm{~mol} \%(\mathrm{n}=3)$ were performed under $\mathrm{Ca}^{2+}$-free conditions. 
Moreover, the functional reconstitution of syt-1 into LUVs was corroborated by a control experiment in which syt-1 doped proteo-LUVs were monitored by CLSM to bind to proteinfree PSMs containing $2 \mathrm{~mol} \% \mathrm{PI}(4,5) \mathrm{P}_{2}$ and $10 \mathrm{~mol} \%$ PS in the absence of $\mathrm{Ca}^{2+}$. For LUVs without syt-1, however, docking to the protein-free PSM was not observed.

As a next step, after the verification of functional reconstitution of syt-1 into LUVs by means of RIfS and CLSM, the direct insertion method was used to co-reconstitute syt-1 and syb 2 into the same LUV population. The successful co-reconstitution of syt-1 and syb 2 was confirmed by density centrifugation in a Nycodenz gradient, the resulting SDS-PAGE analysis is depicted in Figure 4-22.

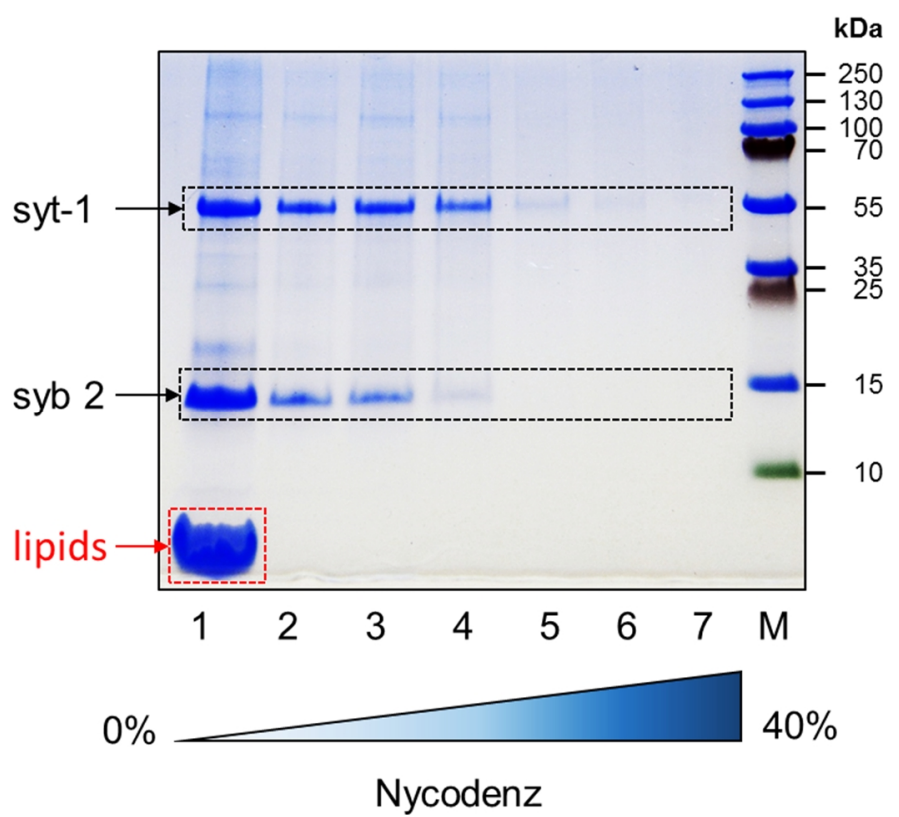

Figure 4-22 Nycodenz assay to analyse the co-reconstitution of syt-1 ( $p / l=1: 1000)$ and syb $2(p / l=1: 500)$ into LUVs by the direct insertion method. Fractions (1-7) were taken from top down after ultracentrifugation of the syb 2/ syt-1/ LUV-suspension in a Nycodenz gradient (0-40\% (w/v)) to segregate proteoliposomes from unreconstituted protein. SDS-PAGE analysis of the taken samples (1-7) verified successful co-reconstitution of syt-1 and syb 2 into LUVs by showing the most prominent bands in fraction 1 with the lowest Nycodenz density and highest lipid content.

However, a successful co-reconstitution of syt-1 and syb 2 into LUVs does not imply that syb 2 is also reconstituted functionally by this method. To confirm functional reconstitution of syb 2 by the direct insertion method, vesicle-vesicle bulk fusion experiments were performed as detailed in Chapter 3.3.3. The time-resolved curve of the FRET-acceptor's fluorescence intensity of an exemplary bulk fusion experiment between SUVs doped with $\triangle$ N49 $(\mathrm{p} / \mathrm{l}=1: 500)$ and LUVs containing syb $2(\mathrm{p} / \mathrm{l}=1: 500)$ and syt- $1(\mathrm{p} / \mathrm{l}=1: 1000)$ as well as the corresponding control experiment are shown in Figure 4-23. For all fusion experiments that were performed in the presence of syt-1, the membranes harboring syt-1 and syb 2 were composed of PC/PE/PS/Chol/TxR-DPPE (55:19:5:20:1), whereas the membranes containing 
the counterpart, $\Delta \mathrm{N} 49$, were composed of $\mathrm{PC} / \mathrm{PE} / \mathrm{PS} / \mathrm{PI}(4,5) \mathrm{P}_{2} / \mathrm{Chol} /$ Atto488-DPPE $(48: 19: 10: 2: 20: 1)$.

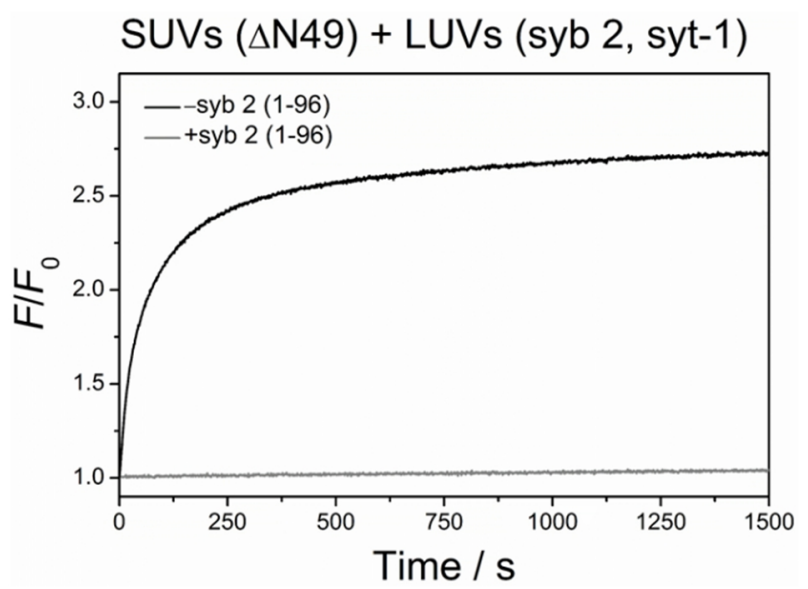

Figure 4-23 Time-resolved fluorescence intensity curve of a vesicle-vesicle bulk fusion experiment of SUVs ( $\triangle$ N49) with LUVs (syb 2, syt-t) (black) and the corresponding control experiment, for which the $\Delta$ N49 doped SUVs were incubated with syb 2 (1-96) prior to fusion (grey). The acceptor complex $\Delta$ N49 was reconstituted in a ratio of $\mathrm{p} / \mathrm{l}=1: 500$ using the co-micellization method, whereas the co-reconstitution of syb $2(\mathrm{p} / \mathrm{l}=1: 500)$ and syt-1 $(\mathrm{p} / \mathrm{l}=1: 1000)$ into LUVs was achieved by applying the direct insertion method.

The bulk fusion experiment and the corresponding blind experiment, in which the $\Delta \mathrm{N} 49$ doped SUVs were incubated with the $\Delta$ N49-blocking fragment syb 2(1-96) prior to the fusion experiment, clearly demonstrate SNARE-specific fusion and therefore functional reconstitution of syb 2 into LUVs by the direct insertion method.

After verification of functional co-reconstitution of syb 2 and syt-1, bulk fusion experiments under three different conditions were performed to investigate the impact of the $\mathrm{Ca}^{2+}$-sensor syt-1 on SNARE-mediated fusion. Firstly, as a standard, vesicle-vesicle fusion in the absence of $\mathrm{Ca}^{2+}$ and ATP was analyzed. Secondly, bulk fusion experiments in the presence of $\mathrm{Ca}^{2+}$ $\left(c\left(\mathrm{Ca}^{2+}\right)=100 \mu \mathrm{M}\right)$ were carried out and thirdly, bulk fusion experiments in the presence of $\mathrm{Ca}^{2+}\left(c\left(\mathrm{Ca}^{2+}\right)=100 \mu \mathrm{M}\right)$ and ATP $(c($ ATP $)=5 \mathrm{mM})$. Normalized, time-resolved courses of the FRET-acceptor's fluorescence intensity during SNARE-mediated bulk fusion under these three different conditions are shown in Figure 4-24. In all depicted cases, the $\Delta$ N49 complex was reconstituted in a protein to lipid ratio of $\mathrm{p} / 1=1: 500$ using the co-micellization method, whereas syb $2(\mathrm{p} / 1=1: 500)$ and syt-1 $(\mathrm{p} / 1=1000)$ were co-reconstituted by the direct insertion method. From the bulk fusion measurements under standard conditions $\left(-\mathrm{Ca}^{2+},-\mathrm{ATP}\right)$, it became obvious that the presence of syt-1 increases the kinetics of vesicle-vesicle bulk fusion by the factor of 2 in comparison to SNARE-mediated SUV-SUV bulk fusion in the absence of syt-1 (cf. Chapter 4.2). However, this syt-1 induced increase in the kinetics was already observed under $\mathrm{Ca}^{2+}$-free conditions and was not enhanced by the addition of $\mathrm{Ca}^{2+}$. Quite contrary to the 
expectation, the kinetics of vesicle-vesicle bulk fusion were even decreased in the presence of $\mathrm{Ca}^{2+}$. Here, the $\mathrm{Ca}^{2+}$ evoked reduction in the bulk fusion kinetics was more pronounced in case of fusion between GUVs and LUVs (Figure 4-24B) than between SUVs and LUVs (Figure 4-24A) where only a negligible difference was observed.

A

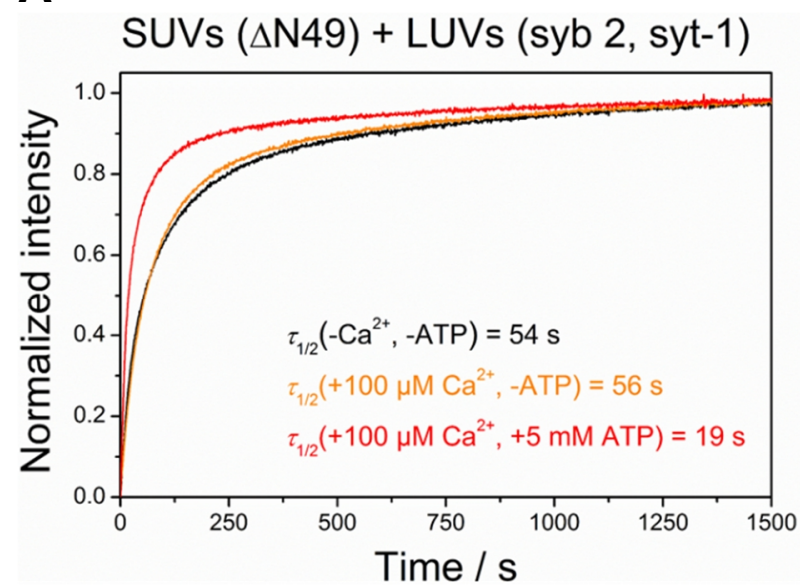

B

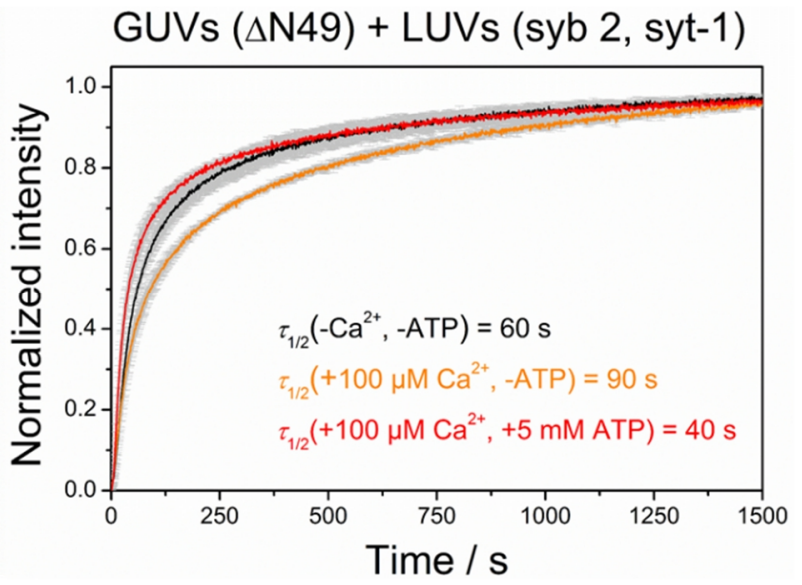

Figure 4-24 Normalized and time-resolved courses of the FRET-acceptor's (TxR-DPPE) fluorescence intensity in SNARE-mediated vesicle-vesicle bulk fusion experiments. (A) Bulk fusion experiments of proteo-SUVs $\left(\Delta\right.$ N49) with proteo-LUVs (syb 2, syt-1) under different conditions leading to values for $\tau_{1 / 2}$ of

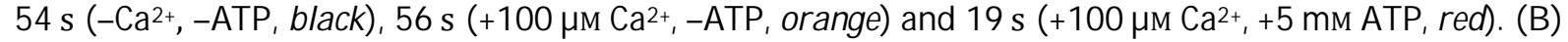
Average curves of bulk fusion experiments of proteo-GUVs $(\Delta N 49)$ with proteo-LUVs (syb 2, syt-1) under different conditions leading to values for $\tau_{1 / 2}$ of $60 \mathrm{~s}$ (-Ca ${ }^{2+},-\mathrm{ATP}$, black), $90 \mathrm{~s}$ ( $+100 \mu \mathrm{m} \mathrm{Ca}{ }^{2+},-\mathrm{ATP}$, orange) and $40 \mathrm{~s}\left(+100 \mu \mathrm{m} \mathrm{Ca}{ }^{2+},+5 \mathrm{~mm}\right.$ ATP, red). Two experiments were performed under each condition, the error bars of the average curves are depicted in grey.

Only by adding ATP additionally to $\mathrm{Ca}^{2+}$, a further increase in vesicle-vesicle fusion kinetics was observed. The addition of ATP and $\mathrm{Ca}^{2+}$ in combination increased the bulk fusion kinetics by a factor of 2.8 in case of SUV-LUV fusion and by a factor of 1.5 in case of GUV-LUV fusion in comparison to the standard conditions.

As a significant increase in bulk fusion kinetics induced by syt-1 was observed, the next challenge was to confirm this fusion behavior on the single vesicle level. To this end, PSMs with reconstituted $\Delta \mathrm{N} 49$ and a $\mathrm{PI}(4,5) \mathrm{P}_{2}$ content of $2 \mathrm{~mol} \%$ were prepared (cf. Chapter 3.2.6) as well as LUVs doped with syb 2 and syt-1 using the direct insertion method. The fusion between these two distinct membrane systems on the single vesicle level was analyzed by means of dual color confocal laser scanning fluorescence microscopy under the same three different conditions as applied for bulk fusion experiments. The statistical analysis of the recorded single vesicle events under each condition are detailed in the following. 


\section{Statistical analysis of single vesicle fusion in the absence of $\mathrm{Ca}^{2+}$}

Firstly, the impact of syt- 1 on single vesicle fusion under standard conditions $\left(-\mathrm{Ca}^{2+},-\mathrm{ATP}\right)$ was investigated. In total, 241 single vesicle events were detected in the recorded time series and evaluated. The analysis resulted in a fusion efficiency of $46 \pm 5 \%$ which in turn means that $54 \pm 6 \%$ of the vesicles that docked to the PSM were stalled in the docking state and did not proceed to fusion within the time window of observation. Among the $46 \%$ of the vesicles that fused with the PSM, $74 \%$ proceeded to full fusion, whereas $26 \%$ remained in an intermediate fusion state. The underlying data for this analysis was extracted from 6 recorded time series on different PSM patches and the resulting statistics are depicted in Figure 4-25A. Furthermore, 65 docking lifetimes were obtained from the recorded time traces which are shown in the histogram in Figure 4-25B.

A

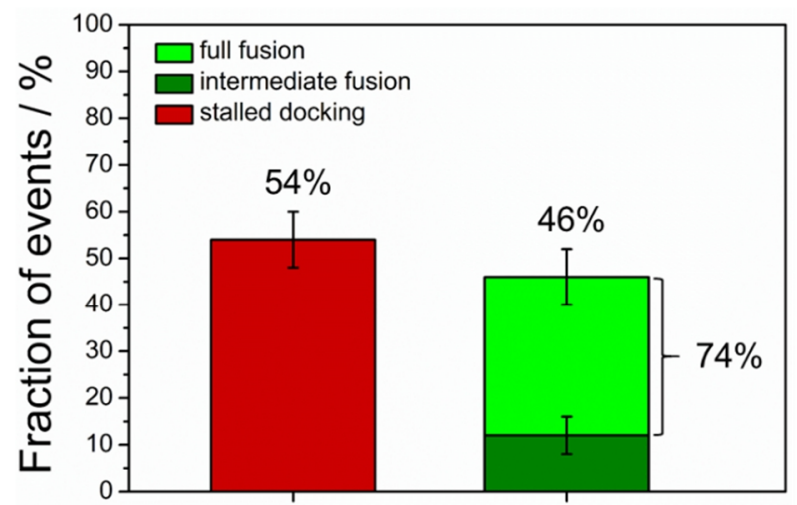

Docking

Fusion
B

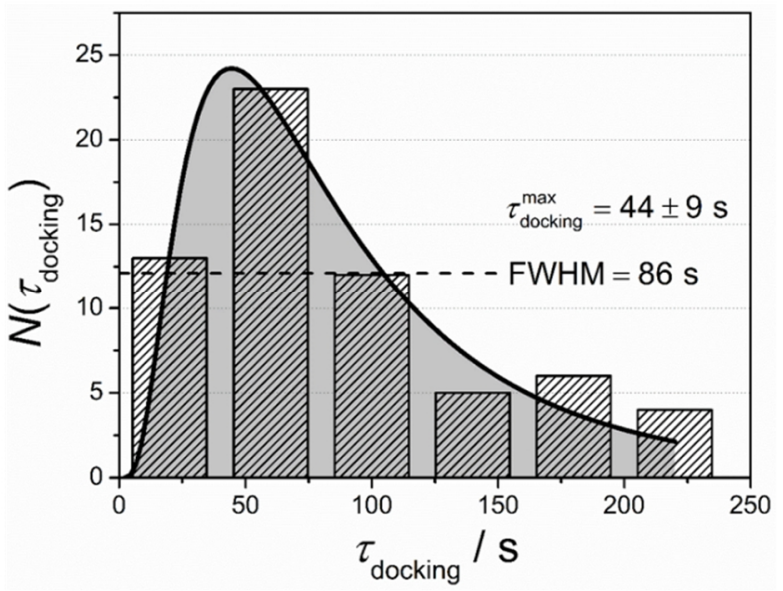

Figure 4-25 Analysis of the fusion behavior of syt- 1 and syb 2 containing LUVs docked to $\Delta$ N49 doped PSMs with a PI $(4,5) \mathrm{P}_{2}$ content of $2 \mathrm{~mol} \%$ under standard conditions (-Ca ${ }^{2+}$, -ATP). (A) The statistical analysis of 241 detected single vesicle events resulted in a mean fusion efficiency of $46 \%$. Among the $46 \%$ of the vesicles that fused with the PSM, $74 \%$ proceeded to full fusion, whereas $26 \%$ remained in an intermediate fusion state. (B) Histogram of lifetimes of the docking state $\tau_{\text {docking }}$ of 65 proteo-LUVs on PSMs. Fitting of a log-normal distribution (Equation (4-1)) to the data resulted in a maximum value for the docking lifetime of $44 \mathrm{~s}$ and a FWHM of $86 \mathrm{~s}$.

A syt-1 induced increase in kinetics as observed in the vesicle-vesicle bulk fusion experiments under standard conditions $\left(-\mathrm{Ca}^{2+},-\mathrm{ATP}\right)$ was not monitored on the single vesicle level. The average lifetimes of the docking state of SNARE-bound LUVs on PSMs with a PI(4,5) $\mathrm{P}_{2}$ content of $2 \mathrm{~mol} \%$ in the presence and absence of syt-1 were determined to be equal with $44 \mathrm{~s}$ (cf. Figure 4-9A). In addition, the fusion efficiency was found to be significantly reduced in the presence of syt-1 (44\%) in comparison to the absence (93\%, cf. Figure 4-8) under $\mathrm{Ca}^{2+}$-free conditions. This raises the question of whether this would be different in the presence of $\mathrm{Ca}^{2+}$ as syt-1 is proposed to be the main $\mathrm{Ca}^{2+}$-sensor of neuronal exocytosis and it is assumed that in 
the presence of $\mathrm{Ca}^{2+}$ syt-1's C2A and C2B domains are able to amplify the tethering between two negatively charged membranes (cf. Chapter 1.4).

\section{Statistical analysis of single vesicle fusion in the presence of $\mathrm{Ca}^{2+}$}

Under $\mathrm{Ca}^{2+}$-free conditions no impact of syt-1 on SNARE-mediated single vesicle fusion was detected concerning the average lifetime of the docking state of SNARE-bound LUVs on PSMs. Further, the integration of syt-1 into the LUVs even resulted in a decreased fusion efficiency by the factor of $\sim 2$. Therefore, the influence of syt-1 on SNARE-mediated single vesicle fusion on PSMs was investigated in the presence of $100 \mu \mathrm{M} \mathrm{Ca}^{2+}$.

In total, 443 single vesicle events were detected in 5 recorded time series. The analysis and evaluation of these time series resulted in a mean fusion efficiency of $38 \pm 5 \%$. Hence, $62 \pm 5 \%$ of the proteoliposomes that docked to the PSM were stalled in the docking state and did not proceed to fusion within the time window of observation. Among the $38 \%$ of the vesicles that fused with the PSM, $99 \%$ proceeded to full fusion, whereas only $1 \%$ remained in an intermediate fusion state. The resulting mean percentages of the fractions of events obtained by a separate analysis of each recorded time series are depicted in Figure 4-26A. Furthermore, 130 docking lifetimes were extracted from the recorded time traces which are shown in the histogram in Figure 4-26B.

A

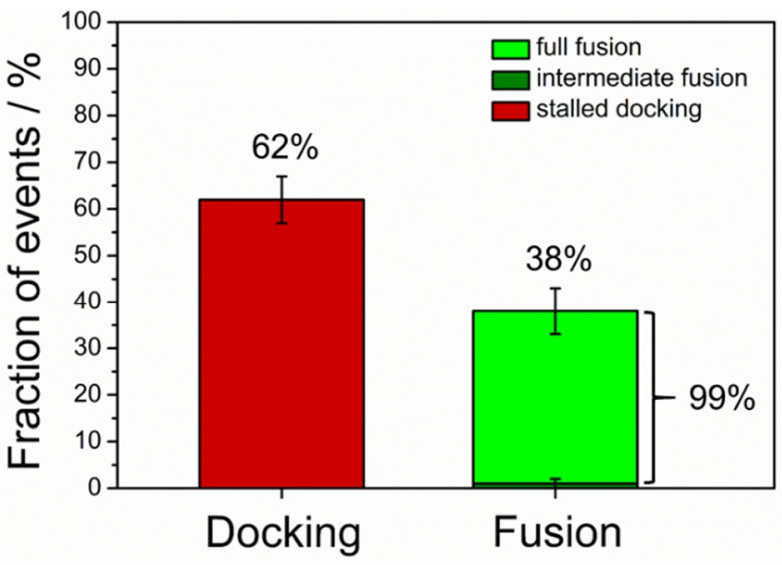

B

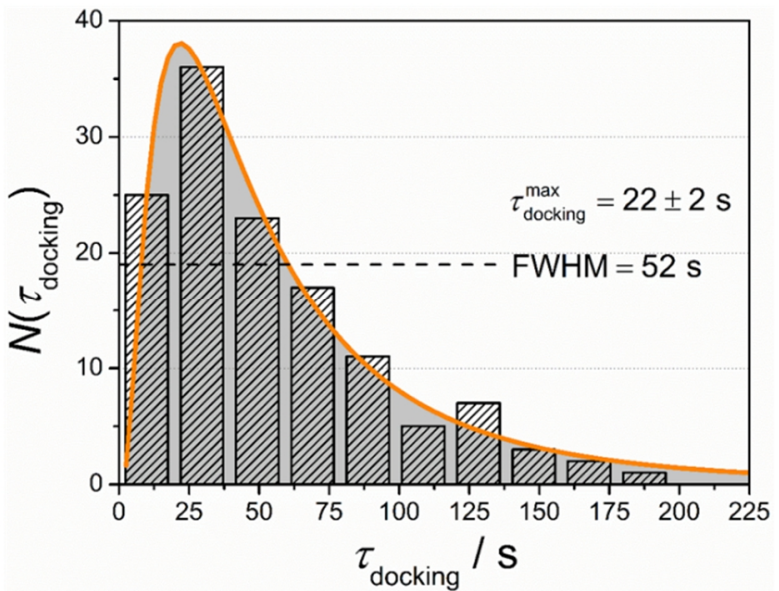

Figure 4-26 Analysis of the fusion behavior of syt- 1 and syb 2 containing LUVs docked to $\Delta$ N49 doped PSMs with a $\mathrm{PI}(4,5) \mathrm{P}_{2}$ content of $2 \mathrm{~mol} \%$ in the presence of $100 \mu \mathrm{M} \mathrm{Ca}{ }^{2+}$. (A) The statistical analysis of 443 detected single vesicle events resulted in a mean fusion efficiency of $38 \%$. Among the $38 \%$ of the vesicles that fused with the PSM, $99 \%$ proceeded to full fusion, whereas only $1 \%$ remained in an intermediate fusion state. (B) Histogram of lifetimes of the docking state $\tau$ docking of 130 proteo-LUVs on PSMs. Fitting of a log-normal distribution (Equation (4-1)) to the data resulted in a maximum value for the docking lifetime of $22 \mathrm{~s}$ and a FWHM of $52 \mathrm{~s}$. 
In the presence of $100 \mu \mathrm{M} \mathrm{Ca}{ }^{2+}$ a clear impact of syt- 1 on the average lifetime of the docking

state was observed. The mode $\tau_{\text {docking }}^{\text {max }}$ of the log-normal distribution (Equation (4-1)) that was fitted to the data shifted to a value of $22 \mathrm{~s}$ which is exactly half of the value obtained in the absence of $\mathrm{Ca}^{2+}$ (cf. Figure 4-25B). The fusion efficiency, however, was further reduced to $35 \%$ but in the presence of $\mathrm{Ca}^{2+}, 99 \%$ of the vesicles that showed fusion proceeded to full fusion - intermediate fusion was nearly non-existent. Most of the detected fusion events (99\%) were categorized as fast full fusion events owing to the complete decay of the $\Omega$-shaped postfusion structure (cf. Figure 4-3) into the PSM within 366 ms or less.

\section{Statistical analysis of single vesicle fusion in the presence of $\mathrm{Ca}^{2+}$ and ATP}

Inspired by the finding that $\mathrm{Ca}^{2+}$-triggered exocytosis in permeabilized neuroendocrine cells was dependent on the presence of ATP, ${ }^{[124]}$ PARK et al. analyzed $\mathrm{Ca}^{2+}$-triggered bulk fusion of purified bovine chromaffin granules with t-SNAREs containing liposomes and observed a significant increase of fusion kinetics and fusion efficiency only in the presence of ATP. They interpreted this observation with an electrostatic mechanism by which the presence of ATP prevents the inactivating cis binding of syt-1 to its own membrane without affecting the activating trans binding to the target membrane. ${ }^{[103]}$ The study of PARK et al. encouraged to investigate the impact of syt-1 on SNARE-mediated single vesicle fusion not only in the presence of $\mathrm{Ca}^{2+}$ but in the presence of $\mathrm{Ca}^{2+}$ and ATP. For this purpose, fusion between syb 2 and syt- 1 containing LUVs and $\triangle \mathrm{N} 49$ doped PSMs were monitored on the single particle level in the presence of $100 \mu \mathrm{M} \mathrm{Ca}^{2+}$ and $5 \mathrm{mM}$ ATP.

Overall, 390 single vesicle events were monitored in 3 recorded time series and evaluated. The separate analysis of each recorded time series led to a mean fusion efficiency of $82 \pm 4 \%$. Consequently, $18 \pm 3 \%$ of the proteo-LUVs that docked to the PSM were observed to be stalled in the docking state and did not proceed to fusion within the time window of observation. Among the $82 \%$ of the vesicles that fused with the PSM, $99 \%$ proceeded to full fusion, whereas only $1 \%$ remained in an intermediate fusion state. The statistical results concerning the mean percentages of the fraction of observed fusion states are depicted in Figure 4-27A. Furthermore, 305 docking lifetimes were extracted from the recorded time traces, the binned numbers are shown in the histogram in Figure 4-27B. 
A

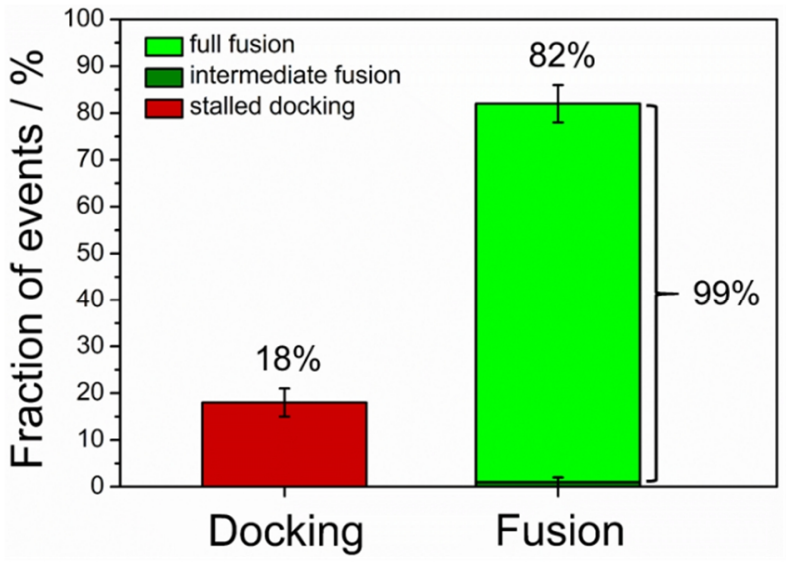

B

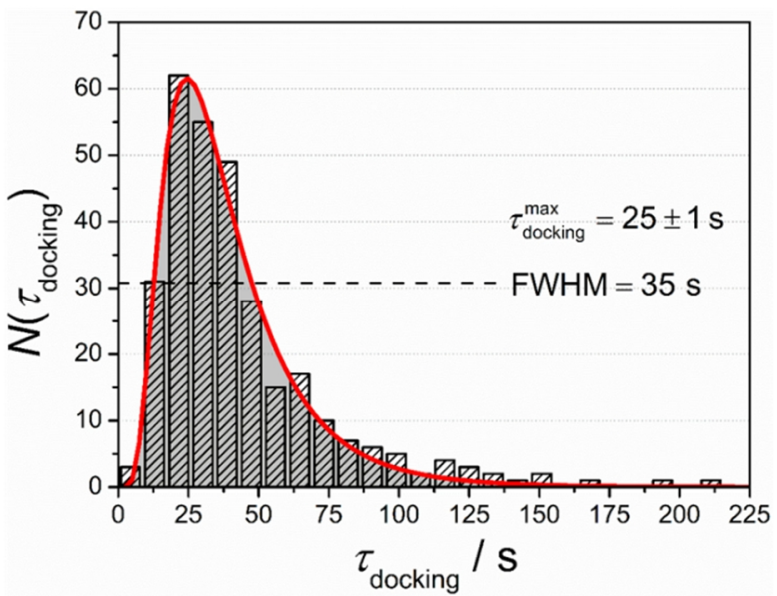

Figure 4-27 Analysis of the fusion behavior of syt- 1 and syb 2 containing LUVs docked to $\Delta$ N49 doped PSMs with a PI $(4,5) \mathrm{P}_{2}$ content of $2 \mathrm{~mol} \%$ in the presence of $100 \mu \mathrm{M} \mathrm{Ca}{ }^{2+}$ and $5 \mathrm{mMATP}$. (A) The statistical analysis of 390 detected single vesicle events resulted in a mean fusion efficiency of $82 \%$. Among the $82 \%$ of the vesicles that fused with the PSM, $99 \%$ proceeded to full fusion, whereas only $1 \%$ remained in an intermediate fusion state. (B) Histogram of lifetimes of the docking state $\tau_{\text {docking }}$ of 305 proteo-LUVs on PSMs. Fitting of a log-normal distribution (Equation (4-1)) to the data resulted in a maximum value for the docking lifetime of $22 \mathrm{~s}$ and a FWHM of $52 \mathrm{~s}$.

The average docking lifetime determined for SNARE-mediated single vesicle fusion in the presence of $\mathrm{Ca}^{2+}$ and ATP remained nearly the same as monitored in the presence of $\mathrm{Ca}^{2+}$ alone, which still means half of the average docking lifetime under standard conditions $\left(-\mathrm{Ca}^{2+},-\mathrm{ATP}\right)$. But with regard to the fusion efficiency, the addition of ATP aside from $\mathrm{Ca}^{2+}$ had a substantial impact on SNARE-mediated single vesicle fusion in the presence of syt-1. An increased fusion efficiency from $38 \%\left(+\mathrm{Ca}^{2+},-\mathrm{ATP}\right)$ to $82 \%\left(+\mathrm{Ca}^{2+},+\right.$ ATP $)$ was observed. The stalled intermediate fusion state was also under these conditions nearly non-existent $(1 \%)$ and the remaining $99 \%$ were classed as fast full fusion events.

\subsection{Verification of fusion pore formation by content labeling}

The assay to analyze SNARE-mediated fusion on the single vesicle level applied in the context of this work is based on the detection of lipid mixing between proteoliposomes and PSMs by means of dual color confocal fluorescence microscopy. In order to respond to justified criticism that fusion assays based on lipid mixing not clearly provide evidence for a fusion pore formation, ${ }^{[17],[125]}$ control fusion experiments of content labeled proteoliposomes with PSMs were performed. In this variant of the assay, the $\Delta \mathrm{N} 49$ doped PSMs were prepared as usual (cf. Chapter 3.2.6) containing Atto488-DPPE as fluorescent marker. The proteo-LUVs with reconstituted syb 2, however, were filled with a Sulforhodamine B containing buffer as a fluorescent content label instead of integrating the membrane marker TxR-DPPE (cf. Chapter 
3.2.5). Time-resolved fluorescence intensity traces of five types of single vesicle fusion events observed in these experiments are shown in Figure 4-28. A docking event was characterized by a sharp increase in the magenta channel (Sulforhodamine B intensity) to a constant intensity level until the end of the time window of observation, whereas the green channel (Atto488DPPE intensity) remained unaffected (Figure 4-28A). While monitoring lipid mixing and content release simultaneously, three different kinds of intermediate fusion were detected. The first kind (Figure 4-28B), most probably a hemifusion, starts with the docking of the proteoLUV followed by a sharp increase in the green channel but lacking the synchronous decrease in the magenta channel. Such a kind of intensity course can be explained by the merger of only the proximal leaflets leading to an Atto488-DPPE influx into the outer leaflet of the LUV and as no content is released during this stage, the magenta channel remains on a constant level. The second kind of intermediate fusion (Figure 4-28C) shows a synchronous increase in the green channel and a decrease in the magenta channel. Here, the green intensity rapidly reaches a saturation level and stays constant until the end of the recording. Exactly the opposite is detected in the magenta channel, which sharply decreases to an intensity between the docking and the baseline level and remains constant afterwards. The constant intensity in the green channel indicates a stable $\Omega$-shaped post-fusion structure of the proteo-LUV and the remaining intensity in the magenta channel could be the consequence of a rapid closure of the fusion pore. An exemplary time trace for a third kind of intermediate fusion is depicted in Figure 4-28D. The only difference to the stalled intermediate fusion in Figure 4-28C is the subsequent incomplete collapse of the proteo-LUV's post-fusion structure into the PSM, characterized by the intensity decrease in the green channel to an intensity level higher than the baseline level. Further, the intensity in the magenta channel does not drop down to the baseline level providing evidence for a residual amount of the content marker Sulforhodamine B in the post-fusion structure. This can again be a result of the rapid closure of the fusion pore or can be caused by a very small vesicular enclosure in the proteo-LUV. The last time-resolved intensity trace shown in Figure 4-28 depicts an exemplary course of a full fusion event between a content labeled proteo-LUV and a membrane marked PSM. Here, the docking of $\sim 45 \mathrm{~s}$ is followed by a rapid decrease to the baseline intensity in the magenta channel at the onset of fusion. The intensity in the green channel, at the same time, shows a sharp in- and decrease. This intensity course in the green channel is the result of two overlapping processes, namely the Atto488DPPE influx into the membrane of the LUV and the synchronous collapse of the $\Omega$-shaped post-fusion structure into the PSM within $2 \mathrm{~s}$. 

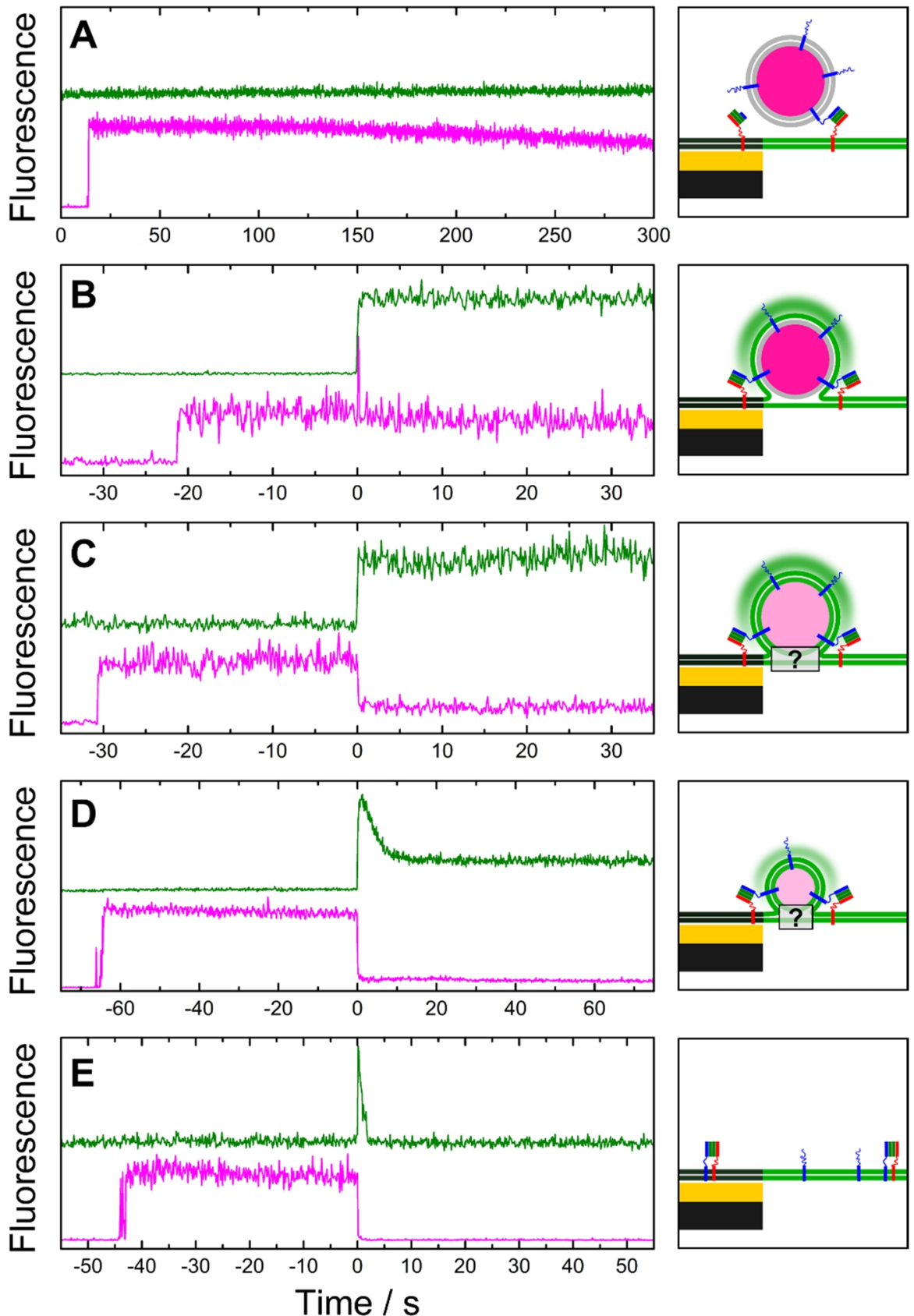

Figure 4-28 (left) Five types of time-resolved intensity traces monitored for SNARE-mediated fusion of content labeled proteo-LUVs (magenta) with membrane labeled PSMs (green) on the single particle level. (right) Corresponding schematic illustrations of the final fusion state the proteo-LUV reached at the end of the recorded time series. (A) Intensity trace of a proteo-LUV stalled in the docking state. (B)-(D) Intensity traces of three types of intermediate fusion. (E) Intensity trace of a full fusion event.

To monitor both lipid mixing and content release upon SNARE-mediated membrane fusion of proteo-LUVs with PSMs was conducted as a control experiment providing evidence that a fusion pore is formed during the fusion process. This proof was necessary to validate the classification of the detected single vesicle fusion events observed only by lipid mixing into the fusion states docking, intermediate fusion and full fusion. However, the combination of detecting content release and lipid mixing simultaneously allows to differ in more detail between distinct intermediate fusion states and should be the way to go for future experiments. 


\subsubsection{Analysis of the influx of Atto488-DPPE into the vesicle}

As in case of content labeled proteo-LUVs no TxR-DPPE was integrated into the vesicle membrane, the increase in the green channel, representing the Atto488-influx from the PSM into the vesicle membrane, was not influenced by FRET. This fact made it worthwhile to analyse the time-dependent intensity traces of the green channel in more detail to gain information about the diffusion behavior of the fluorescent dye Atto488-DPPE through the fusion stalk into the vesicle. Time traces of single vesicle fusion events were selected which showed an intermediate fusion state with a stable $\Omega$-shape for a time interval of at least $5 \mathrm{~s} \hat{=} 40$ frames (cf. Figure 4-28 B+C). A first order exponential equation (Equation (4-5)) was determined to fit the time-resolved intensity courses very good, indicating a one-step mechanism of this process with a specific time constant $t_{1}$. The fluorescence intensity was normalized $(0 \rightarrow 1)$ prior to the analysis, resulting in $y_{0}=1$, whereas $B_{1}$ remained a variable parameter.

$$
f(x)=B_{1} \cdot \exp \left(-\frac{x}{t_{1}}\right)+y_{0}
$$

The fit derived time constant $t_{1}$ was multiplied with $\ln (2)$ to obtain the respective half-life $\tau_{1 / 2}^{1}$ for the Atto488-DPPE influx through the stalk into the vesicle membrane. The evaluation of 22 time traces resulted in a mean half-life for this process of $\bar{\tau}_{1 / 2}^{1}=0.121 \pm 0.065 \mathrm{~s}$ indicating a fast and unhindered lipid diffusion through the stalk. An exemplary time trace fitted with Equation (4-5) as well as a boxplot of the obtained half-lives are depicted in Figure 4-29.

A

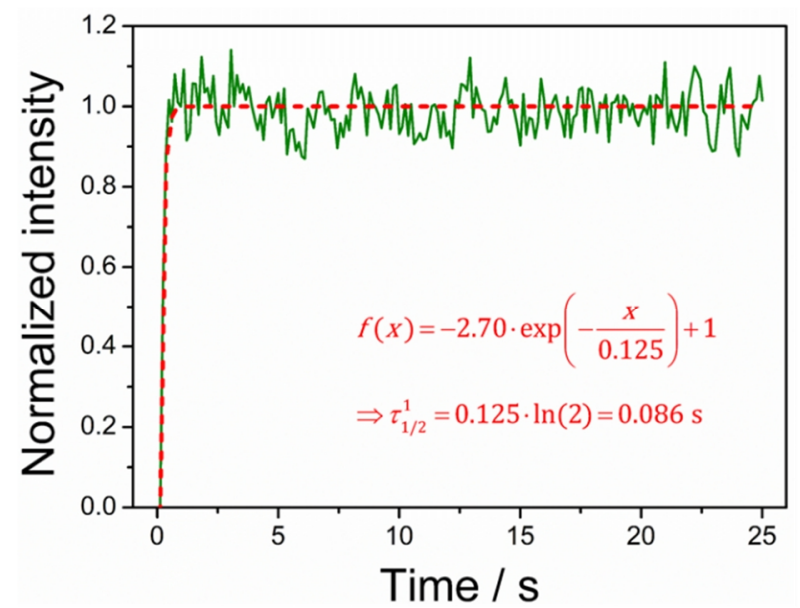

B

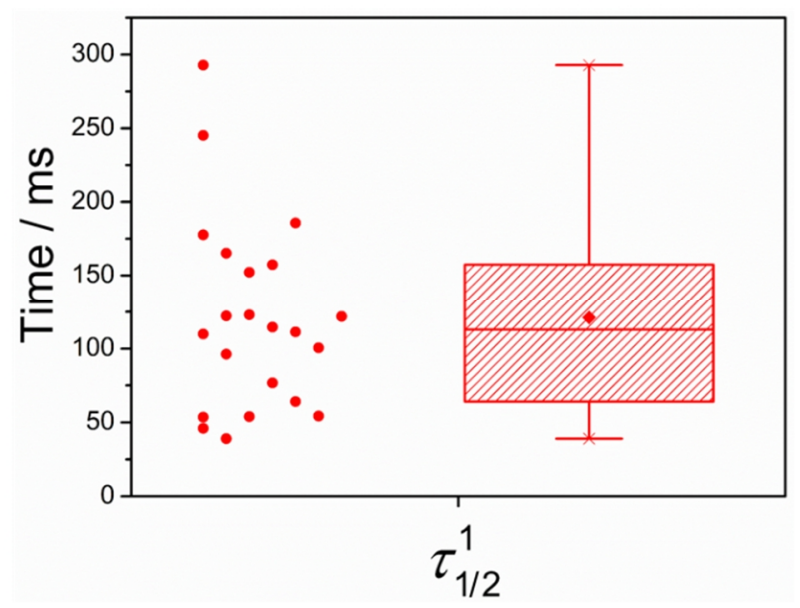

Figure 4-29 (A) Normalized time trace of the green channel (Atto488-DPPE intensity) of a single vesicle fusion event with a stable $\Omega$-shape, stalled in the intermediate fusion state (green line). A first order exponential Equation (4-5) was fitted to the data (red dashed line) and resulted in a half-life of $\boldsymbol{\tau}_{\mathbf{1} / \mathbf{2}}^{\mathbf{1}}=0.086 \mathrm{~s}$ for the Atto488-DPPE influx. (B) Boxplot of 22 obtained half-lives for this one-step process yielded a mean half-life for the Atto488-DPPE influx from the PSM into the vesicle membrane of $\overline{\boldsymbol{\tau}}_{\mathbf{1} / \mathbf{2}}^{\mathbf{1}}=0.121 \pm 0.065 \mathrm{~s}$. 


\subsection{Characterization of the fusion of chromaffin granules with pore-spanning membranes}

In addition to the analysis of SNARE-mediated fusion of synthetic vesicles with PSMs, the fusion behavior of natural chromaffin granules (CGs) with PSMs was investigated. CGs are dense-core secretory granules that are located in the medulla of adrenal glands. They are filled with neuroactive peptides and hormones - like adrenaline and noradrenaline - and play a central role in the fight-or-flight response (cf. Chapter 1.5). Under conditions of fear or stress, CGs fuse with the plasma membrane of chromaffin cells to release their neuroactive content into the bloodstream. For this fusion process, CGs own the same set of SNAREs as synaptic vesicles do and they are known to exhibit a great variety of distinct fusion modes depending on stimulus intensities (cf. Figure 1-12). ${ }^{[79],[86],[126]}$ SNARE-mediated fusion of these natural secretory granules with synthetic PSMs was analyzed to work out similarities and differences of the fusion process in comparison to the synthetic vesicle fusion and to explore the different modes of CG fusion (kiss-and-run, cavicapture and full fusion) in vitro. For this purpose, CGs were isolated from bovine adrenal glands as detailed in Chapter 3.2.8 and labeled with the lipophilic fluorophore $\mathrm{DiD}-\mathrm{C}_{18}$ which inserts into the outer leaflet of the $\mathrm{CG}$ membrane and makes them accessible for the investigation by confocal fluorescence microscopy. $\triangle \mathrm{N} 49$ containing PSMs were prepared as described in Chapter 3.2.6 - fluorescently marked with Atto488-DPPE and with a PI(4,5) $\mathrm{P}_{2}$ content of $2 \mathrm{~mol} \%$. Single particle fusion events between CGs and PSMs were monitored by dual color CLSM. Figure 4-30 shows schematic fluorescence time traces and cross sections of a CG fusion event on the solid supported part of the PSM (s-PSM) to provide guidance for the following interpretation of the recorded time traces of single CG fusion events. The event starts with the docking of the CG to the PSM (1). This leads to a rapid increase in the red channel as the outer leaflet of the CG membrane is labeled with DiD- $\mathrm{C}_{18}$. At the onset of fusion between the CG and the PSM (2), the lipids and therefore the fluorescent markers of the distinct membranes start to mix. This leads to a decrease of the fluorescence intensity in the red channel as DiD- $\mathrm{C}_{18}$ diffuses out of the CG membrane into the PSM and is quenched by the underlying gold layer. At the same time, Atto488-DPPE diffuses into the CG membrane until a saturation is reached (3) and is de-quenched due to an increased distance to the gold layer. Unfortunately, the CGs were not provided with a fluorescent content marker, therefore no reliable conclusions can be made regarding the time of the merger of the distal leaflets leading to the formation of a fusion pore. However, content release - a consequence of fusion pore formation - is accompanied by shrinking of the $\Omega$-shaped post fusion structure of the CG. This collapse of the $\Omega$-shape upon merging of the CG membrane into the PSM results in a decrease 
in the green channel to a certain level $(4+5)$ as the de-quenching effect of the underlying gold layer is height-dependent. ${ }^{[127],[128]}$
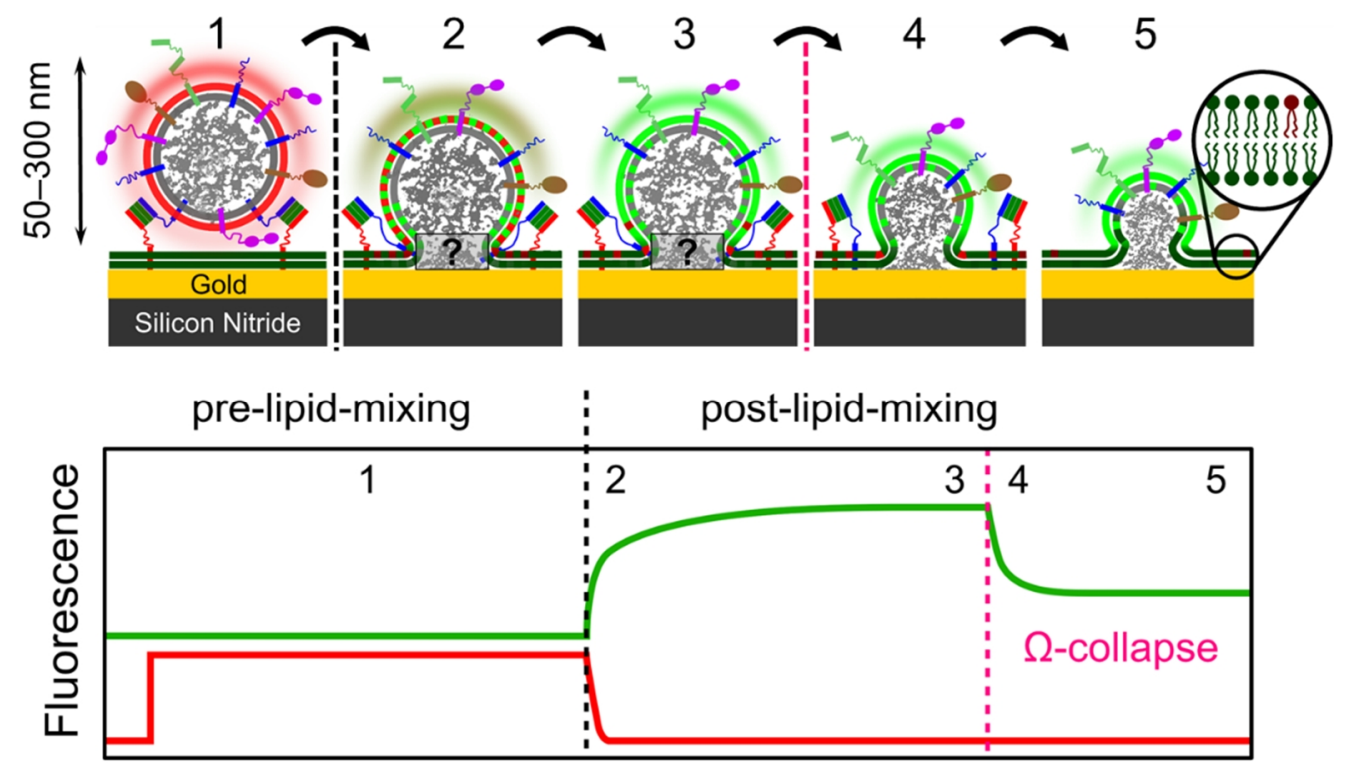

Time

Figure 4-30 Schematic illustration of fluorescent time traces (bottom) and cross sections (top) of a hypothetic CG fusion event on the s-PSM part. The event starts with a DiD-C 18 labeled CG docked to an Atto488-DPPE marked PSM (1). Upon fusion, DiD-C 18 diffuses out of the CG membrane into the PSM and is quenched while Atto488-DPPE simultaneously diffuses into the CG membrane and is de-quenched $(2+3)$. Due to the lack of a CG content marker, no reliable conclusion can be made regarding the time of the merger of the distal leaflets leading to fusion pore formation. A subsequent decrease in the green channel, however, is caused by a content release induced collapse of the $\Omega$-shaped CG post fusion structure $(\mathbf{4 + 5})$ clearly indicating fusion pore formation.

In the following, three types of fluorescence intensity traces that were mainly observed for single CG fusion events on $\Delta \mathrm{N} 49$ containing PSMs with a PI(4,5) $\mathrm{P}_{2}$ content of $2 \mathrm{~mol} \%$ will be presented and evaluated. Figure 4-31 shows exemplary, time-resolved fluorescence intensity traces for these three types of fusion between CGs and PSMs, which mainly differ in the time when the $\Omega$-shaped CG post fusion structure starts to collapse into the PSM. In order to verify that the monitored fusion between CGs and PSMs is SNARE-specific, a control experiment was conducted in which the $\Delta \mathrm{N} 49$ complex was blocked with the v-SNARE fragment syb 2 (1-96) prior to the reconstitution - resulting in PSMs with inactive t-SNARE complexes. In this experiment neither fusion nor docking of the CGs to the PSMs was observed, confirming SNARE-specificity. 

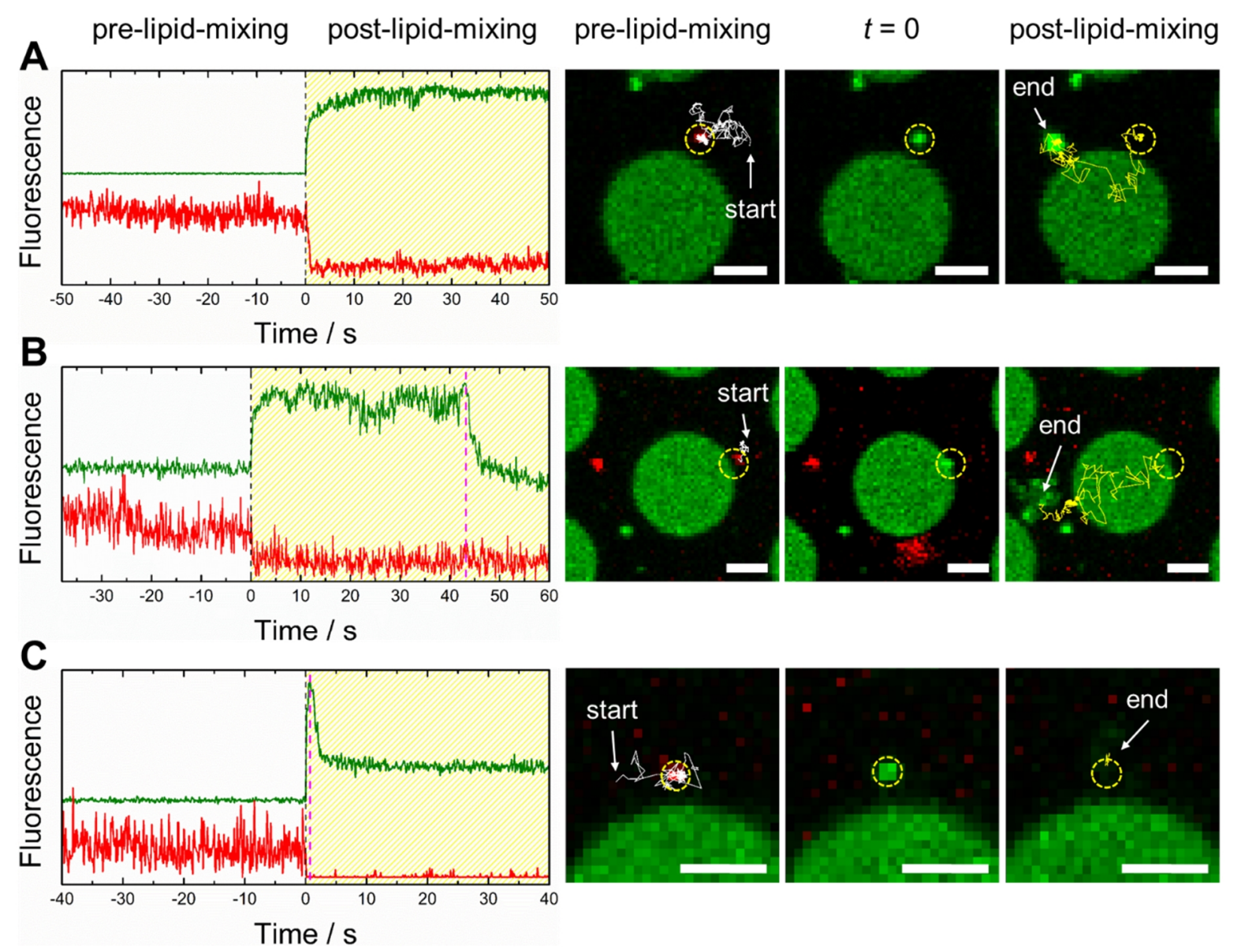

Figure 4-31 (left) Time-resolved fluorescence intensity traces of DiD-C $\mathrm{C}_{18}$ marked CGs (red) fusing with Atto488-DPPE labeled PSMs (green) with reconstituted $\Delta$ N49. (right) Fluorescence micrographs of the corresponding intensity curves on the left, showing the last frame before the onset of lipid mixing (pre-lipidmixing), the frame when lipid mixing starts $(t=0)$ as well as the last frame of the time series (post-lipidmixing). The diffusion trajectories of the CGs before and after lipid mixing are depicted in white and yellow, respectively. The location where lipid mixing between the two membrane systems starts, is highlighted with a dashed, yellow circle. (scale bars: $2 \mu \mathrm{m}$ ) (A) Fusion event with a stable post-lipid-mixing $\Omega$-shape. (B) Fusion event with a delayed collapse of the $\Omega$-shape after lipid mixing (onset marked with a dashed, magenta line). (C) Fusion event with an immediate collapse of the $\Omega$-shape after the onset of lipid mixing.

In 20 recorded time series from 4 independent experiments $290 \mathrm{CG}$ fusion events were detected and evaluated. 170 (59\%) of these events exhibited a stable $\Omega$-shape after the onset of lipid mixing, that did not collapse until the end of the recorded time series, leading to fluorescence intensity traces as shown in Figure 4-31A. 100 (34\%) of the detected CG fusion events showed a delayed collapse of the $\Omega$-shape as depicted exemplarily in Figure 4-31B, whereas in the remaining 20 (7\%) CG fusion events the $\Omega$-shape started to collapse immediately (within $610 \mathrm{~ms} \triangleq 5$ frames) after the onset of lipid mixing as shown in Figure 4-31C. To gather information about CG fusion kinetics, the lifetime of the docking state $\left(\tau_{\text {docking }}\right)$ as well as the lifetime of the $\Omega$-shape before the onset of collapse $\left(\tau_{\Omega 1}\right)$ were extracted from the fluorescence intensity traces. Time-resolved fluorescence intensity traces of an exemplary CG fusion event that captures these steps is shown in Figure 4-32. 


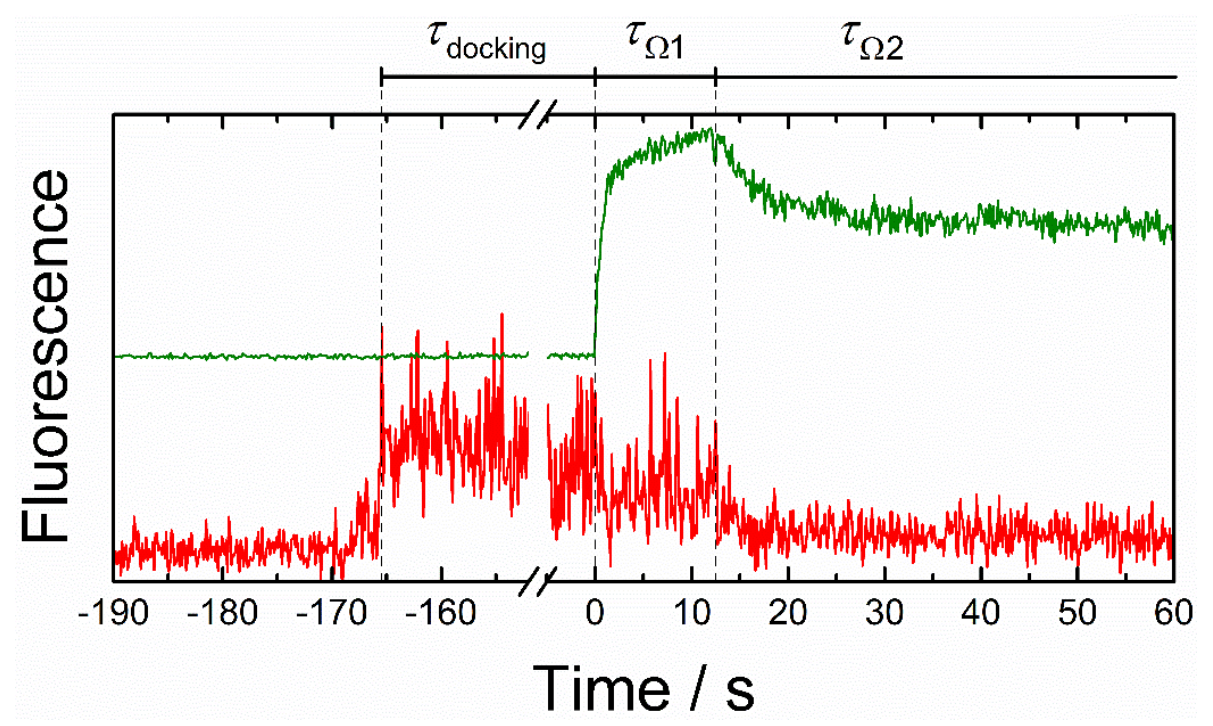

Figure 4-32 Exemplary time-resolved fluorescence intensity traces of a CG fusion event from which the lifetime of the docking state ( $\tau_{\text {docking}}$ ) and the lifetime of the $\Omega$-shape before the onset of collapse $\left(\tau_{\Omega 1}\right)$ were extracted. In this case, the fluorescence intensity in the green channel does not drop down to the baseline level during collapse of the $\Omega$-shape. For this reason, the time for the complete collapse of the vesicle ghost into the PSM $\left(\tau_{\Omega 1}+\tau_{\Omega 2}\right)$ cannot be determined.

Histograms of the determined values for $\tau_{\text {docking }}$ and $\tau_{\Omega 1}$ are depicted in Figure 4-33. A lognormal distribution (Equation (4-1)) was fitted to the data of the obtained docking lifetimes. The evaluation of 104 extracted docking lifetimes resulted in a value for $\tau_{\text {docking }}^{\max } 48 \mathrm{~s}$ and in a FWHM of the log-normal distribution of $85 \mathrm{~s}$ (Figure 4-33A). To the histogram of the determined lifetimes of the $\Omega$-shape before the onset of fusion, the mono exponential Equation (4-6) was fitted.

$$
N\left(\tau_{\Omega 1}\right)=N_{0} \cdot \exp \left(-k_{\Omega 1} \cdot \tau_{\Omega 1}\right)
$$

with $N_{0}$, the total number of CG post fusion structures where the $\Omega$-shape started to decay and $k_{\Omega 1}$, the rate constant for the onset of decay of the $\Omega$-shape. The average lifetime of the $\Omega$-shape before the onset of decay $\bar{\tau}_{\Omega 1}$ is defined as the reciprocal of $k_{\Omega 1}$. The analysis of 98 determined values for $\tau_{\Omega 1}$ led to a rate constant $k_{\Omega 1}$ for the vesicle ghost to start collapsing into the PSM of $0.24 \mathrm{~s}^{-1}$ and an average lifetime of the intact $\Omega$-shape $\bar{\tau}_{\Omega 1}$ of $4.2 \mathrm{~s}$ (Figure 4-33B). A total collapse of the $\Omega$-shape, leading to finite values for $\tau_{\Omega 2}$, was only observed in 10 cases and either took place at the pore edge or within the pore (f-PSM). On the s-PSM, however, the vesicle ghost never collapsed completely i.e., the Atto488-DPPE intensity did not return to the baseline level before lipid mixing. 
A

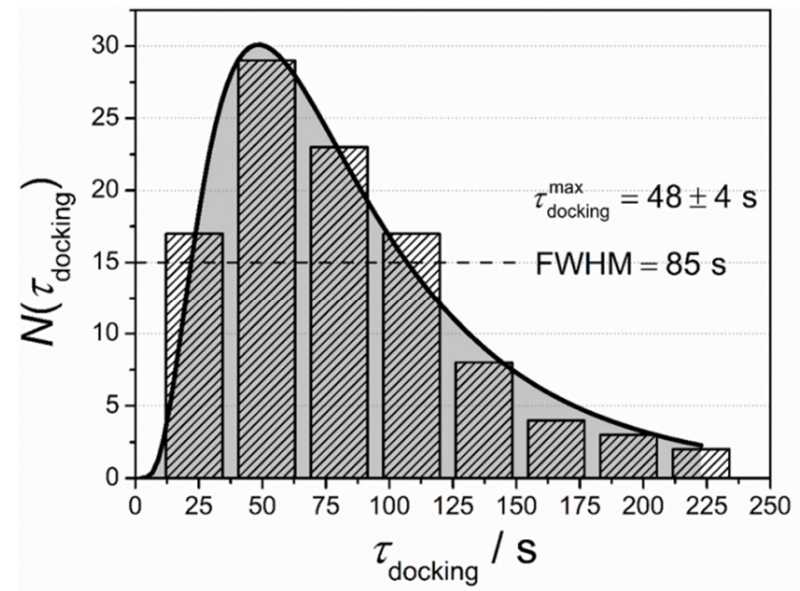

B

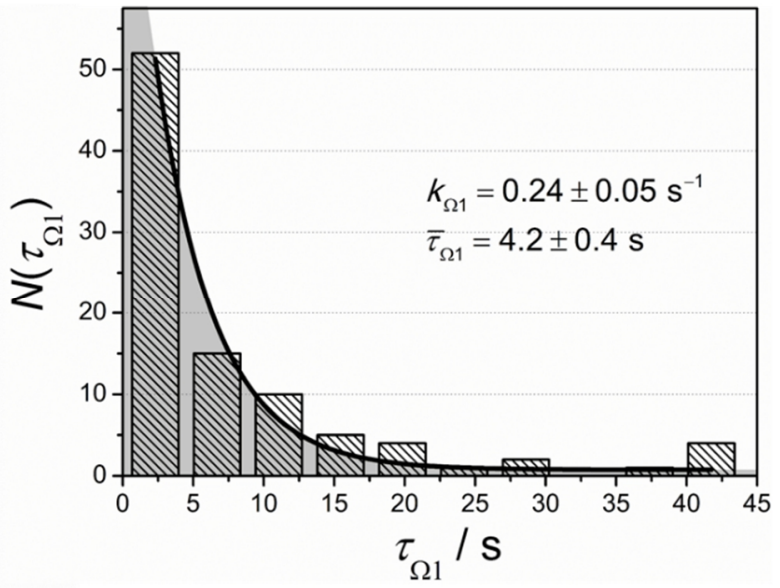

Figure 4-33 (A) Histogram of lifetimes of the docking state ( $\tau_{\text {docking}}$ ) of 104 CGs on PSMs with reconstituted $\Delta \mathrm{N} 49$ and a $\mathrm{PI}(4,5) \mathrm{P}_{2}$ content of $2 \mathrm{~mol} \%$. Fitting of a log-normal distribution (Equation (4-1)) to the data resulted in a maximum value for the docking lifetime of $48 \mathrm{~s}$ and a FWHM of $85 \mathrm{~s}$. (B) Histogram of observed lifetimes of the $\Omega$-shape before the onset of collapse $\left(\tau_{\Omega 1}\right)$ of a total number of 98 detected CG fusion events on PSMs with reconstituted $\Delta \mathrm{N} 49$ and a PI $(4,5) \mathrm{P}_{2}$ content of $2 \mathrm{~mol} \%$. Fitting of a mono exponential decay (Equation (4-6)) to the data resulted in a rate constant $\mathrm{k}_{\Omega 1}$ for the CG post fusion structure starting to collapse into the PSM of $0.24 \mathrm{~s}^{-1}$ and an average lifetime of the intact $\Omega$-shape $\left(\overline{\mathbf{\tau}}_{\mathbf{\Omega 1}}\right)$ of $4.2 \mathrm{~s}$.

\subsubsection{Diffusion behavior of chromaffin granules on pore-spanning membranes}

As set out in the introductory part of the results (Chapter 4.1.2) the mobility of lipids and SNARE-proteins in both parts of the PSM is a significant requirement of the model system to serve for the investigation of SNARE-mediated fusion and it was fulfilled for both. The next question that arises is whether CGs that are docked to the PSM by the formation of one or more SNARE-complexes can retain the mobility on this artificial membrane system as it was observed for plasma membrane bound CGs in living chromaffin cells. ${ }^{[80],[129]-[132]}$ Indeed, as already hinted in Figure 4-31 on the right, part of the docked CGs were monitored to be mobile on the PSM and in contrast to the artificial ones not only on the free-standing part (f-PSM) but also on the solid supported part (s-PSM). Moreover, mobility was not only observed in case of docked CGs before the onset of fusion (pre-lipid-mixing, pre-lm) but even for CGs that already fused with the PSM (post-lipid-mixing, post-lm). Diffusion coefficients for CGs on the s-PSM as well as on the f-PSM before and after lipid mixing were determined with the Matlab-based software TrackNTrace which was developed by STEIN and THIART. ${ }^{[13]}$ Figure 4-34 shows a boxplot of the derived diffusion coefficients. On the s-PSM, mean diffusion coefficients of $\bar{D}_{\text {s-PSM }}\left(\right.$ pre-lm) $=0.12 \pm 0.05 \mu \mathrm{m}^{2} / \mathrm{s}$ and $\bar{D}_{\text {s-PSM }}($ post- $\mathrm{lm})=0.04 \pm 0.03 \mu \mathrm{m}^{2} / \mathrm{s}$ were obtained, whereas on the f-PSM, the diffusion coefficients were determined to be larger with values for the mean of $\bar{D}_{\text {f-PSM }}($ pre- $\mathrm{lm})=0.34 \pm 0.06 \mu \mathrm{m}^{2} / \mathrm{s}$ and $\bar{D}_{\text {f-PSM }}($ post- $\mathrm{lm})=0.40 \pm 0.13 \mu \mathrm{m}^{2} / \mathrm{s}$. 


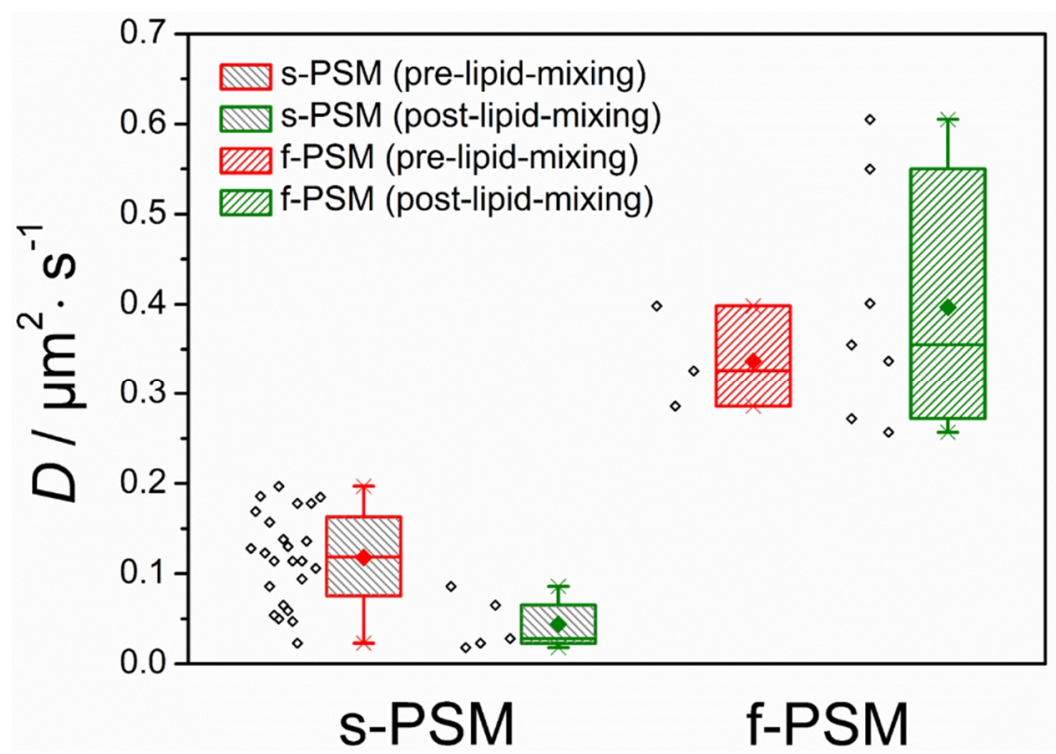

Figure 4-34 Boxplot of diffusion coefficients of CGs pre and post-lipid-mixing with diffusion trajectories separated on both parts of the PSM, the solid supported part (s-PSM) and the free-standing part (f-PSM).

The diffusion coefficients presented in Figure 4-34 were obtained from diffusion trajectories separated on the two distinct parts of the PSM. But in fact, the CG diffusion was not necessarily restricted by the pore boundaries. In many cases, pre- as well as post-lipid-mixing, the CGs were able to move across the pore boundaries as exemplarily shown in Figure 4-35 and Figure 4-36. Caused by the fact that CGs were able to cross pore boundaries, trajectories with a sufficient length to determine reliable diffusion coefficients on the distinct parts of the PSMs were rare (the only exception being pre-lipid-mixing CG diffusion on the s-PSM). The tracking and diffusion analysis of docked CGs before lipid mixing was executed in the red channel utilizing the fluorescent probe DiD- $\mathrm{C}_{18}$ in the outer leaflet of the $\mathrm{CG}$ membrane. Due to DiD-C 18 entry into the PSM upon CG fusion and a slight crosstalk of the Atto488-DPPE into the red channel, the segmentation into f-PSM and s-PSM was also visible in the red channel and got the more pronounced the more fusion occurred. An example for unconfined diffusion of a SNARE-bound CG on a PSM before lipid mixing is shown in Figure 4-35. The mean square displacement (MSD) of the CG plotted against time intervals of up to $36.6 \mathrm{~s}$ is depicted in Figure 4-35B and highlights the unrestricted diffusion behavior in this case. In Figure 4-35C the MSD of the CG is plotted against time intervals of up to $1342 \mathrm{~ms}$. A linear regression was fitted to the data to obtain the CG diffusion coefficient $D=0.088 \mu \mathrm{m}^{2} / \mathrm{s}$ from the slope $(4 D)$. 


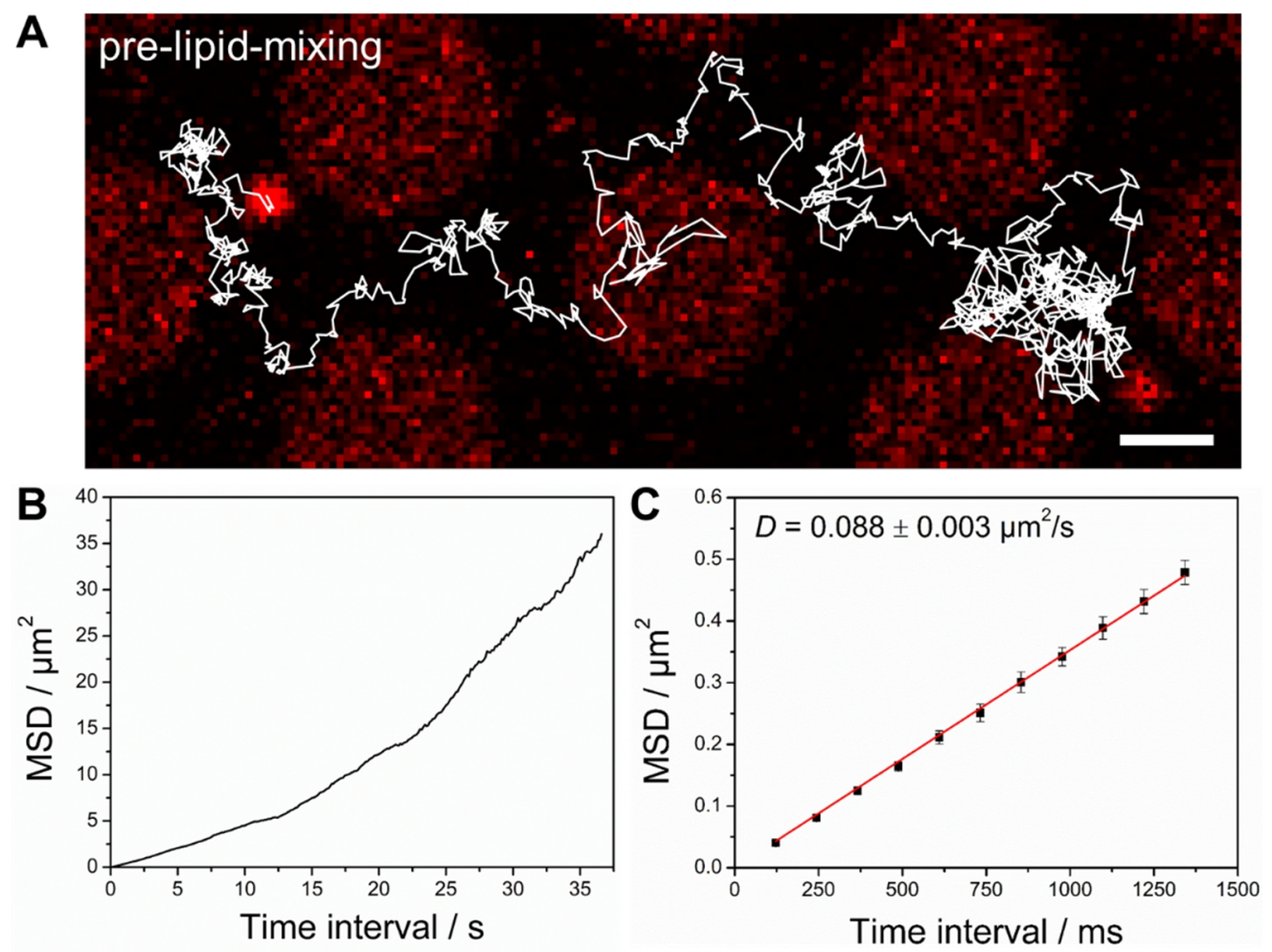

Figure 4-35 (A) Fluorescence micrograph of the last frame of an exemplary pre-lipid-mixing CG diffusion on a PSM across pore boundaries. In case of CG diffusion before lipid mixing, the CGs were tracked in the red channel based on the DiD- $\mathrm{C}_{18}$ fluorescence intensity. The diffusion trajectory is depicted as a white line. (scale bar: $2 \mu \mathrm{m}$ ) (B) Plot of the mean square displacement (MSD) of the CG against time intervals of up to $36.6 \mathrm{~s}$ showing an unconfined diffusion behavior. (C) Plot of the MSD of the CG against time intervals of up to $1342 \mathrm{~ms}$. The diffusion coefficient $\mathrm{D}=0.088 \mu \mathrm{m}^{2} / \mathrm{s}$ was obtained from the slope (4D) of the linear regression line (red).

It should be noted however that unrestricted CG diffusion before lipid mixing was not observed in every case but only in some, giving proof of the principle possibility. For tracking and diffusion analysis of diffusing CGs on PSMs after lipid mixing, the green channel was utilized as the PSM marker Atto488-DPPE flowed into the CG membrane upon fusion. If the CG fused with the PSM within a pore (f-PSM), high mobilities with reliable diffusion coefficients of up to $0.6 \mu \mathrm{m}^{2} / \mathrm{s}$ were observed for post-lipid-mixing CG diffusion as long as the CG kept distance from the pore edge. At the pore edge, the CG post-fusion structure often got stuck and remained there. However, in some cases the CG post-fusion structure was able to detach from the pore edge to move again through the pore to the next pinning point at the pore edge or even to cross it at a certain position to continue diffusion on the s-PSM part. An example for such a diffusion behavior of a CG after lipid mixing which scans the pore edge for an exit to get on the s-PSM part is shown in Figure 4-36. 

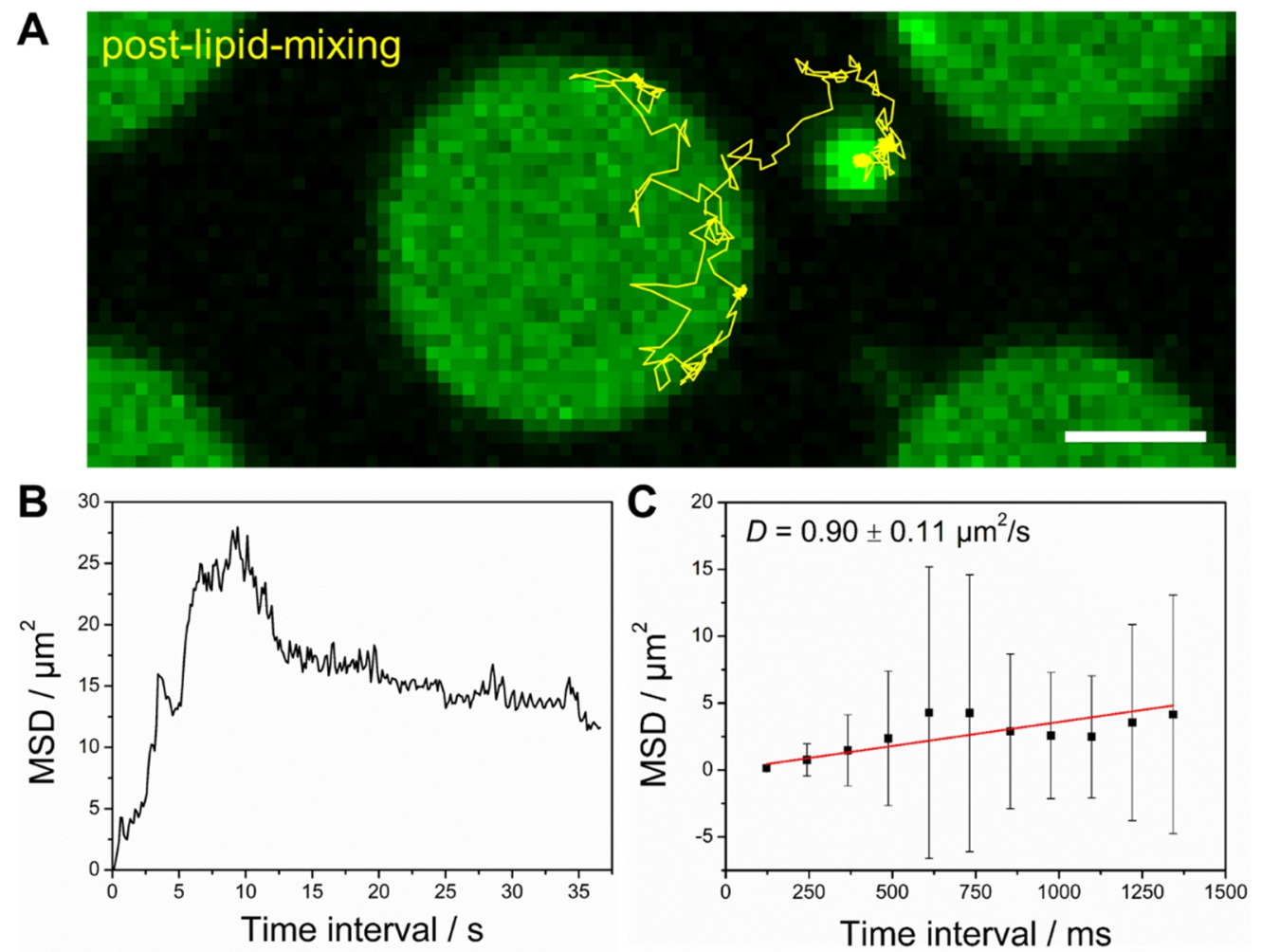

Figure 4-36 (A) Fluorescence micrograph of the last frame of an exemplary post-lipid-mixing CG diffusion on a PSM scanning the pore edge for an exit to continue diffusion on the s-PSM. In case of CG diffusion after lipid mixing, the CGs were tracked in the green channel based on the Atto488-DPPE fluorescence intensity. The diffusion trajectory is depicted as a yellow line. (scale bar: $2 \mu \mathrm{m}$ ) (B) Plot of the mean square displacement (MSD) of the CG against time intervals of up to $36.6 \mathrm{~s}$ showing a confined diffusion behavior and ultimately got stuck on the s-PSM. (C) Plot of the MSD of the CG against time intervals of up to $1342 \mathrm{~ms}$. The diffusion coefficient $\mathrm{D}=0.90 \mathrm{\mu m}^{2} / \mathrm{s}$ was obtained from the slope (4D) of the linear regression line (red), however, it is not reliable due to an unsteady diffusion getting stuck at pinning points at the pore edge and on the s-PSM.

The property to be able to move across the pore boundaries of a PSM is a unique feature of CG diffusion and was not observed for synthetic vesicles on PSMs, not in the docked state nor in the intermediate fusion state. Moreover, synthetic vesicles were monitored to be completely immobile on the s-PSM part in a SNARE-bound state as detailed in studies of KUHLMANN ${ }^{[45],[90]}$ and also observed in this work. This unique diffusion behavior of CGs on PSMs made it worthwhile to analyze it in more detail, especially with the focus on the probability density of the CG's location on the distinct parts of the PSM in case of pre- as well as post-lipid-mixing diffusion. To tackle this issue, the PSM was divided into trigonal unit cells with the centers of three pores at each edge of the triangle (Figure 4-37). The pore centers were taken as a reference location to determine the CG's location as the shortest distance to the next pore center. This results in a CG catchment area $A$ of $1 / 3$ of the whole unit cell area for each pore center as schematically depicted in Figure 4-37A. 
A

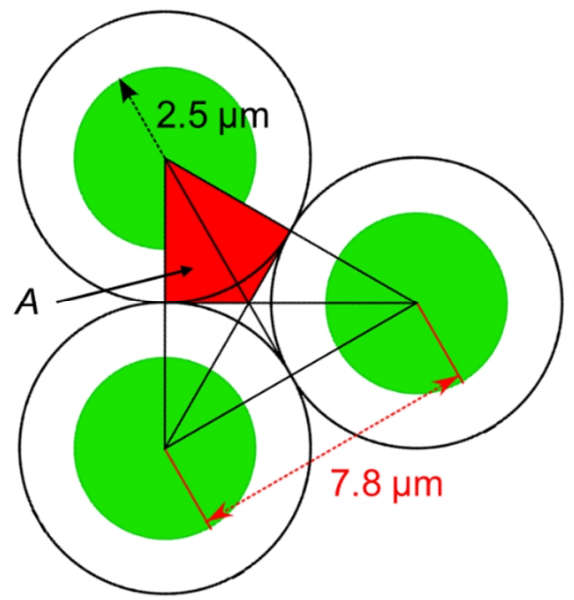

B

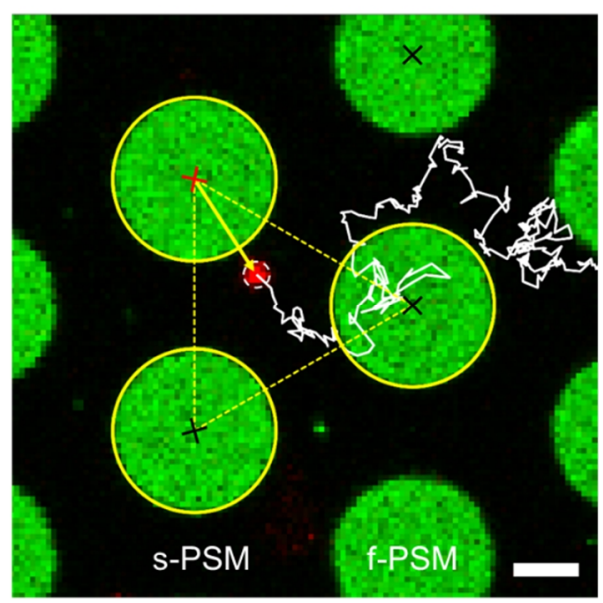

Figure 4-37 (A) Schematic illustration of the triangular unit cell used for CG-pore center distance determinations (pore radius: $2.5 \mu \mathrm{m}$; pore center distance (pcd): $7.8 \mu \mathrm{m}$ ). $1 / 3$ of the unit cell, corresponding to the catchment area A of one pore center in this unit cell is depicted in red. (B) Fluorescence micrograph of a CG (red) diffusing on a PSM (green) with the diffusion trajectory depicted as a white line and the relevant unit cell as a dashed, yellow triangle. The pore center with the shortest distance to the CG is highlighted in red. (scale bar: $2 \mu \mathrm{m}$ ).

With a Matlab-based software written by I. MEY, all extracted CG diffusion trajectories were analyzed regarding their distance of every single location to the next pore center. The determined CG-pore center distances $r_{\mathrm{x}}$ were divided by the pore radius $\left(r_{\text {pore }}=2.5 \mu \mathrm{m}\right)$, leading to relative distances. Given by the highly regular geometry of the porous substrate, the maximum distance between a CG and the next pore center that can be achieved - marked by the center of the triangular unit cell - is $r_{\max }=3.9 \mu \mathrm{m} / \cos \left(30^{\circ}\right)=4.5 \mu \mathrm{m}$. This results in a maximum relative distance of $r_{\max } / r_{\text {pore }}=1.8$. All relative distances with values for $r_{\mathrm{x}} / r_{\text {pore }} \leq 1$ were therefore assigned to CG locations inside the pore (f-PSM) and all distances with values $1<r_{\mathrm{x}} / r_{\text {pore }} \leq 1.8$ to CG locations on the rim (s-PSM). For pre-lipid-mixing CG diffusion, 218 diffusion trajectories from 8 different time series were evaluated, resulting in a total number of 92358 determined CG-pore center distances. A histogram of the obtained relative CG-pore center distances counted in bin sizes of 0.04 is shown in Figure 4-38A. In case of post-lipidmixing CG diffusion, 45 trajectories from 9 different time series were analyzed, leading to a total number of $43466 \mathrm{CG}$-pore center distances. Figure 4-38B shows the histogram of the relative $\mathrm{CG}$-pore center distances post-lipid-mixing counted in bin sizes of 0.04 . 
A

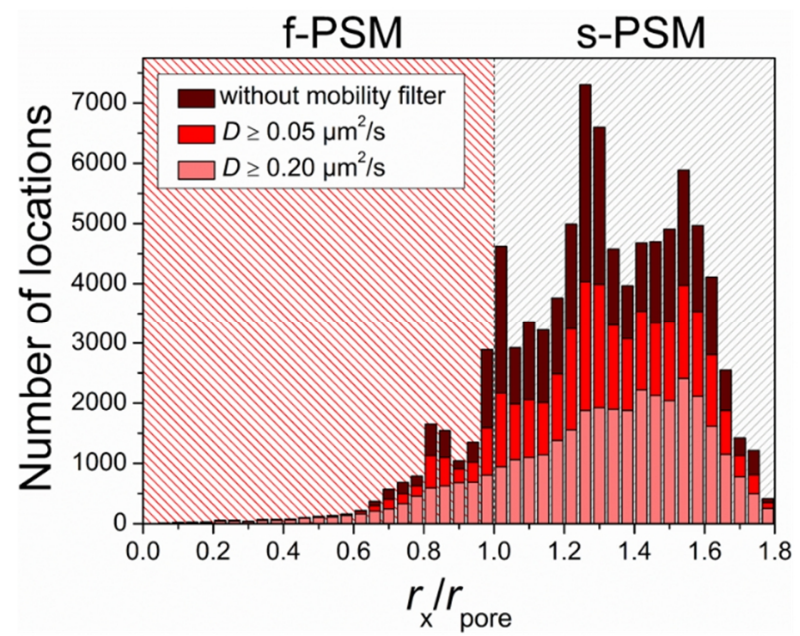

B

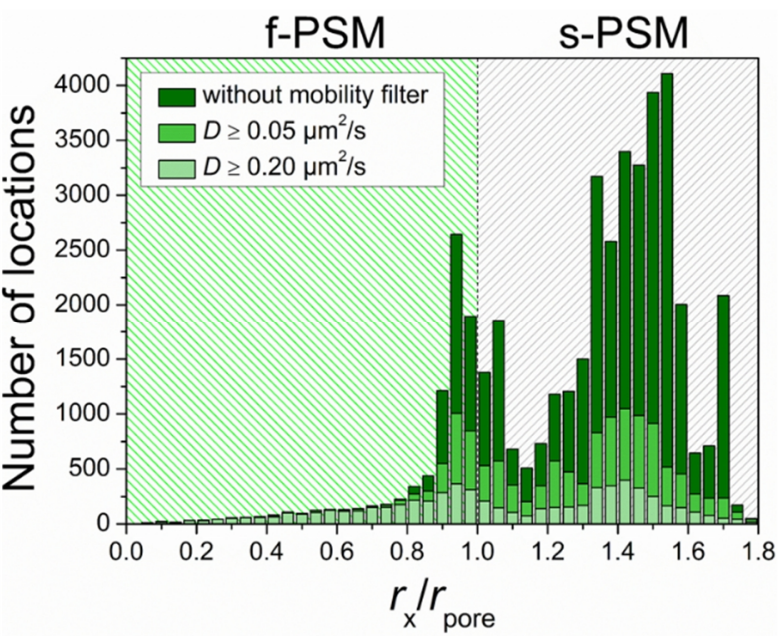

Figure 4-38 Histograms of relative pore center distances of CGs diffusing on a PSM counted in bin sizes of 0.04. (A) Pore center distances of diffusing CGs pre-lipid-mixing. The dark red bars account for the total number of 92358 determined CG-pore center distances, whereas the red and the light red bars represent pore center distances of CGs with a mobility greater than or equal to $0.05 \mu \mathrm{m}^{2} / \mathrm{s}$ and $0.20 \mathrm{\mu m}^{2} / \mathrm{s}$, respectively. (B) Pore center distances of diffusing CGs post-lipid-mixing. The dark green bars account for the total number of 43466 determined CG-pore center distances, whereas the green and the light green bars represent pore center distances of CGs with a mobility greater than or equal to $0.05 \mu \mathrm{m}^{2} / \mathrm{s}$ and $0.20 \mathrm{~mm}^{2} / \mathrm{s}$, respectively.

Diffusing CGs - particular on the s-PSM part but also at the edges of pores - tended to get stuck at certain positions, remained there for a while to than continue to diffuse on the PSM to the next pinning point. Therefore, some CG-pore distances were overdetermined, leading to peaks in the histograms in Figure 4-38 (dark red and dark green bars). The tendency to get stuck was more pronounced in case of $\mathrm{CG}$ diffusion post-lipid-mixing. To correct for the overdetermination of $\mathrm{CG}$-pore center distances caused by stuck CGs, a mobility filter was applied. By determining the distances between consecutive localizations of every extracted CG diffusion trajectory, followed by the calculation of the diffusion coefficient of the CG between these two localizations, all distances that resulted in a CG diffusion coefficient $D<0.05 \mu \mathrm{m}^{2} / \mathrm{s}$ were excluded. The remaining CG-pore center distances were classified as mobile fraction and are depicted as red and green bars in Figure 4-38. For the highly mobile fraction (light red and light green bars in Figure 4-38), the mobility filter was adjusted to exclude all CG-pore center distances with a CG diffusion coefficient $D<0.20 \mu \mathrm{m}^{2} / \mathrm{s}$. The percentages of the different mobility states of diffusing CGs on PSMs pre and post-lipid-mixing are compiled in Table 4-7. 
Table 4-7 Overview of the percentages of mobility states of diffusing CGs on PSMs pre and post-lipidmixing.

\begin{tabular}{lcc}
\hline CG mobility state & Pre-lipid-mixing & Post-lipid-mixing \\
\hline $\mathbf{D}<\mathbf{0 . 0 5} \mathbf{~ m}^{2} / \mathbf{s}$ & $33 \%$ & $66 \%$ \\
$\mathbf{0 . 0 5} \mathbf{~ m}^{2} / \mathbf{s} \leq \mathbf{D}<\mathbf{0 . 2 0} \mathbf{~ m}^{2} / \mathbf{s}$ & $29 \%$ & $19 \%$ \\
$\mathbf{D} \geq \mathbf{0 . 2 0} \mathbf{~ m}^{2} / \mathbf{s}$ & $38 \%$ & $15 \%$ \\
\hline
\end{tabular}

As the distribution of CG mobility states is quite homogeneous pre-lipid-mixing, the tendency to low mobilities is very pronounced in case of CG diffusion post-lipid-mixing.

As a next step, the probability density of CG locations (PD) on PSMs was calculated by dividing the counts of the determined CG-pore center distances $r_{\mathrm{x}}$ through the value of the area of the corresponding catchment segment $A_{\mathrm{x}}$ (cf. Figure 4-39). Starting at the pore center, the first catchment segment was defined as a circular sector with a central angle of $60^{\circ}$ and a radius of $0.1 \mu \mathrm{m}$, followed by circular segments with a gradually increasing radius by steps of $0.1 \mu \mathrm{m}$ as described in Equation (4-7).

$$
A_{\mathrm{x}}\left(r_{\mathrm{x}}\right)=1 / 6 \cdot\left[\pi \cdot r_{\mathrm{x}}^{2}-\pi \cdot\left(r_{\mathrm{x}}-0.1 \mu \mathrm{m}\right)^{2}\right]
$$

Equation (4-7) was used for the calculation of $A_{\mathrm{x}}$ until $r_{\text {crit }}=3.9 \mu \mathrm{m}$ (half of the pore center distance) was reached (cf. Figure 4-37). For CG-pore center distances greater than $r_{\text {crit, }}$ the calculation of $A_{\mathrm{x}}$ was more complex as, in this case, the circular segments of the three pores making up a unit cell, start to overlap. A detailed derivation of the calculation of $A_{\mathrm{x}}$ for $r_{\mathrm{x}}>r_{\text {crit }}$ is shown in the appendix in Chapter 7.2.
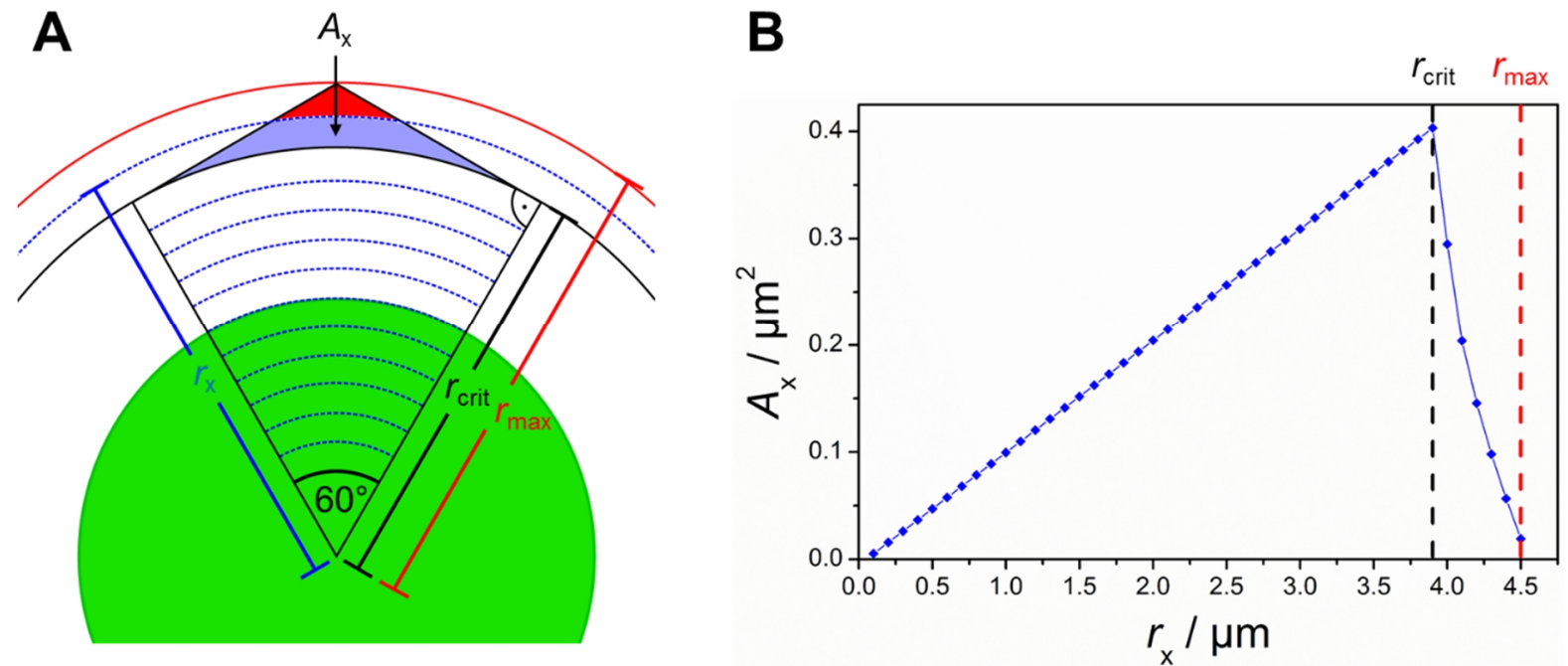

Figure 4-39 (A) Schematic illustration of the catchment area of one pore center of a unit cell subdivided into catchment segments $A_{x}\left(r_{x}\right)$ (marked by the dashed blue lines) needed for the calculation of the probability density of CG locations on PSMs. (B) Plot of the calculated values for $A_{x}\left(r_{x}\right)$ from $r_{x}=0.1-4.5 \mu m$. 
The normalized counts of the relative CG-pore center distances determined for the three different mobility states of a CG diffusing on a PSM as well as the resulting probability densities are shown in Figure 4-40 (pre-lipid-mixing) and Figure 4-41 (post-lipid-mixing). Normalization was carried out by setting the integral of all counts to 1 and $A_{\mathrm{x}}$ was normalized likewise.
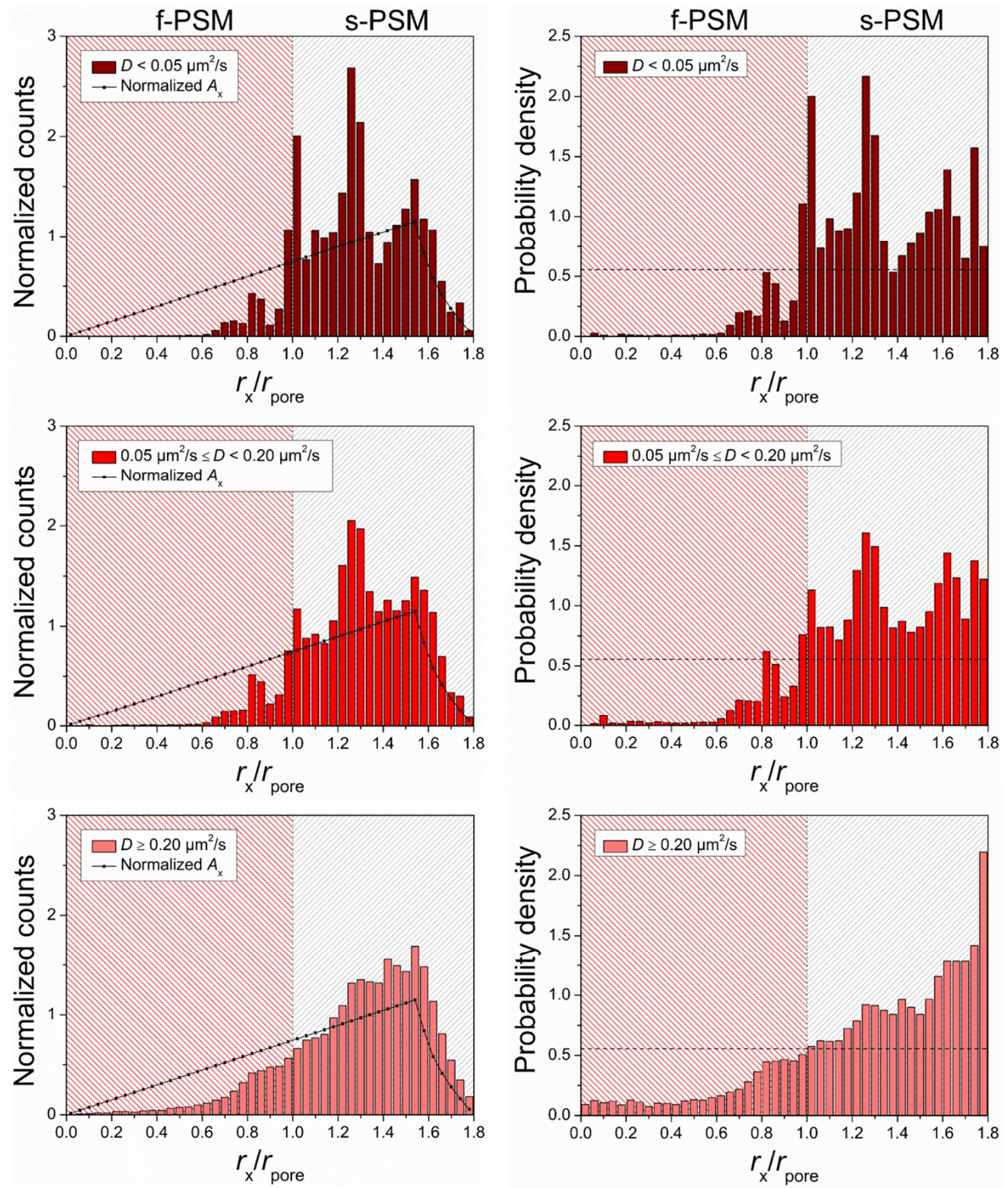

Figure 4-40 (left) Normalized counts of the relative CG-pore center distances for the three different mobility states of diffusing CGs on PSMs pre-lipid-mixing. The normalized catchment area $A_{x}$ for the corresponding bars is plotted as a guide for the eye. (right) Probability densities (PDs) for the three different mobility states of diffusing CGs on PSMs pre-lipid-mixing. The integral under the dashed line from 0 to 1.8 equals 1 as does the sum of the area of all bars. 

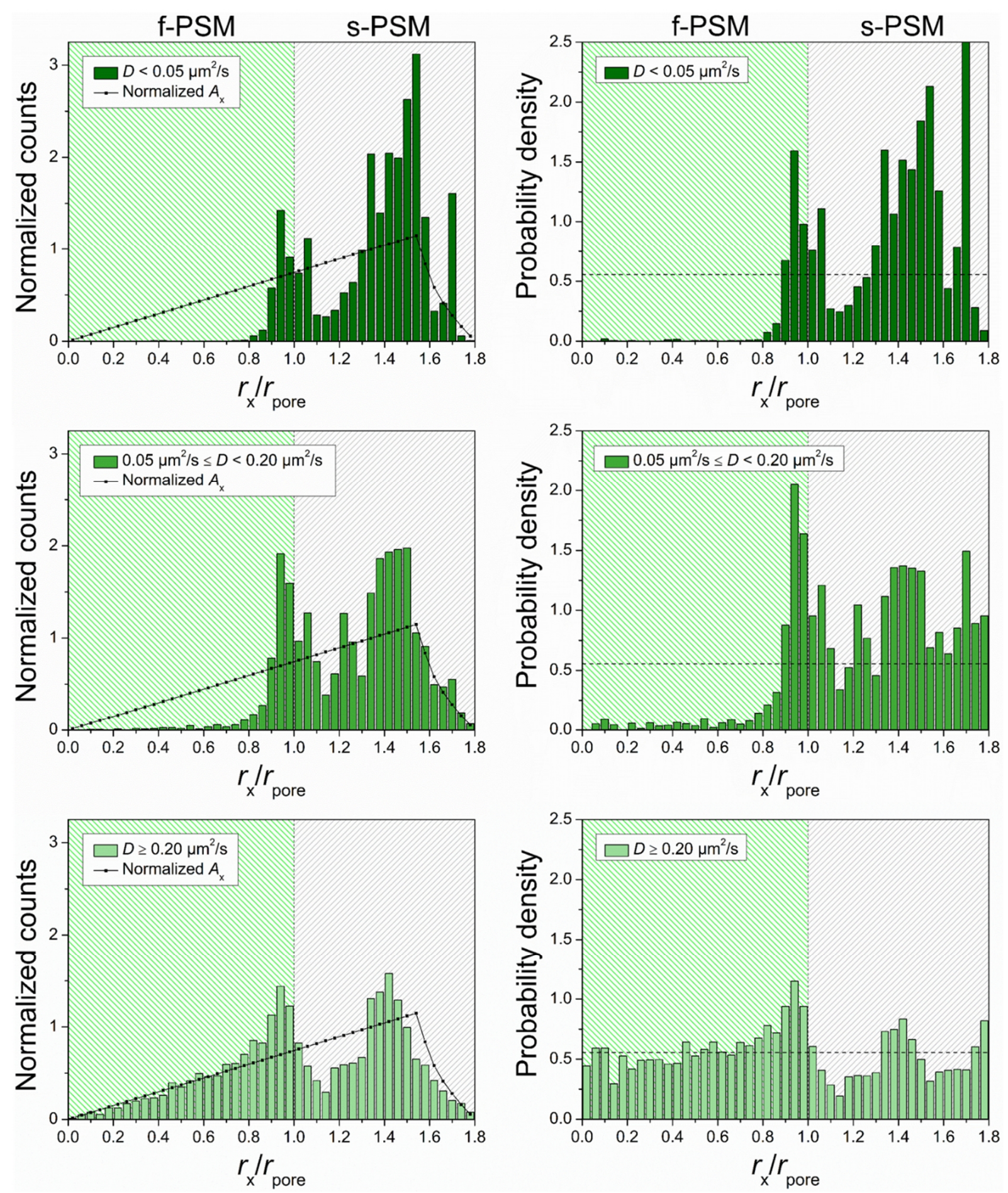

Figure 4-41 (left) Normalized counts of the relative CG-pore center distances for the three different mobility states of diffusing CGs on PSMs post-lipid-mixing. The normalized catchment area $A_{x}$ for the corresponding bars is plotted as a guide for the eye. (right) Probability densities (PDs) for the three different mobility states of diffusing CGs on PSMs post-lipid-mixing. The integral under the dashed line from 0 to 1.8 equals 1 as does the sum of the area of all bars.

To provide an overview on the distribution of the probability density of diffusing CGs on the distinct parts of the PSM, the area under the bars in the f-PSM region $\left(r_{\mathrm{x}} / r_{\text {pore }}=0.0-1.0\right)$ as well as in the s-PSM region $\left(r_{\mathrm{x}} / r_{\text {pore }}=1.0-1.8\right)$ were integrated. The obtained percentages of the CG's PD on the distinct parts of a PSM pre and post-lipid-mixing are shown in Table 4-8. 
Table 4-8 Percentages of the probability density (PD) on the two distinct parts of a PSM (f-PSM and s-PSM) for CG diffusion - pre as well as post-lipid-mixing - in three different mobility states.

\begin{tabular}{lcccc}
\hline \multirow{2}{*}{ CG mobility state } & \multicolumn{2}{c}{ PD (pre-lipid-mixing) } & \multicolumn{2}{c}{ PD (post-lipid-mixing) } \\
& f-PSM & s-PSM & f-PSM & s-PSM \\
\hline $\mathbf{D}<\mathbf{0 . 0 5} \mathbf{~ m m}^{2} / \mathbf{s}$ & $14 \%$ & $86 \%$ & $14 \%$ & $86 \%$ \\
$\mathbf{0 . 0 5} \mathbf{~ m}^{2} / \mathbf{s} \leq \mathbf{D}<\mathbf{0 . 2 0} \mathbf{~ m}^{2} / \mathbf{s}$ & $15 \%$ & $85 \%$ & $25 \%$ & $75 \%$ \\
$\mathbf{D} \geq \mathbf{0 . 2 0} \mathbf{~ m}^{2} / \mathbf{s}$ & $22 \%$ & $78 \%$ & $61 \%$ & $39 \%$ \\
\hline
\end{tabular}

In both cases, pre- and post-lipid-mixing, the trend to increasing percentages of the PD on the f-PSM part can be observed for higher mobility states. While the distribution of the PD on the two distinct parts of a PSM is the same for CG diffusion pre and post-lipid-mixing in the lowest mobility state, it changes considerably with increasing mobility in case of CG diffusion postlipid-mixing. Whereas, in case of CG diffusion pre-lipid-mixing, the changes in PD percentages on both parts of the PSM are very low among the different mobility states (cf. Table 4-8).

\subsubsection{Analysis of the influx of Atto488-DPPE into the CG membrane}

Upon SNARE-mediated fusion between a CG and the PSM it comes to lipid mixing between the two distinct membranes. This includes the diffusion of the PSM marker Atto488-DPPE through the fusion stalk into the CG membrane (cf. Figure 4-30). A closer view and a detailed analysis of the course of influx of Atto488-DPPE into the CG membrane revealed another striking property of SNARE-mediated fusion between CGs and PSMs. The remarkable finding here is the occurrence of a two-step process, starting with a fast influx, represented by a sharp increase in the green channel, which is followed by a second, significantly slower influx leading to a saturation level of the fluorescence intensity in the green channel. Figure 4-42A shows an exemplary, time-resolved fluorescence intensity trace of a CG fusion event on the s-PSM part, exhibiting a stable $\Omega$-shape until the end of the recorded time series. The increase of the fluorescence intensity is induced by the influx of Atto488-DPPE into the CG membrane and a second order exponential Equation (4-8) was fitted to the data to extract the time constants $t_{1}$ and $t_{2}$ for the two steps.

$$
f(x)=B_{1} \cdot \exp \left(-\frac{x}{t_{1}}\right)+B_{2} \cdot \exp \left(-\frac{x}{t_{2}}\right)+y_{0}
$$


A

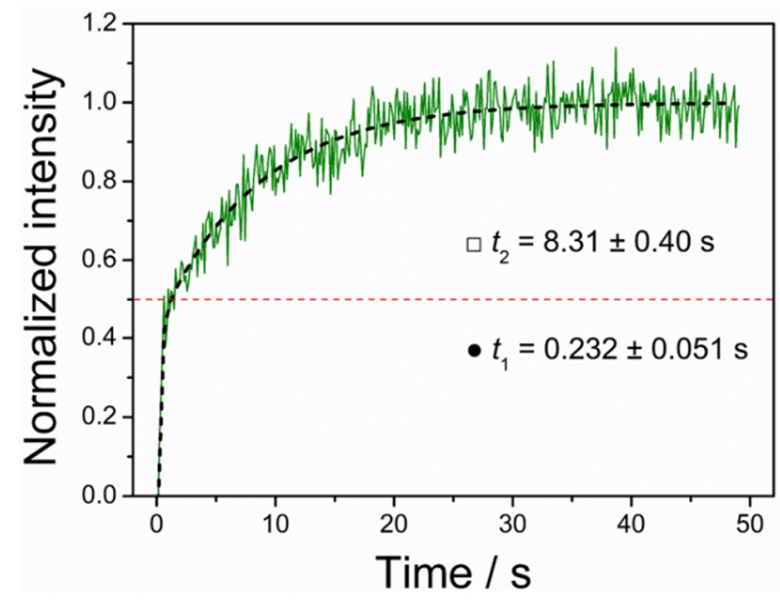

B

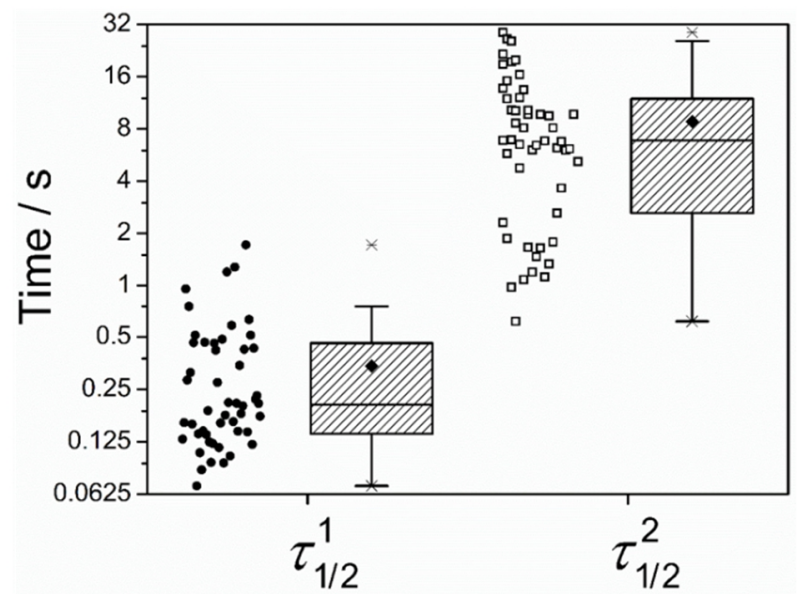

Figure 4-42 (A) Normalized, time-resolved fluorescence intensity trace of a CG fusion event on the s-PSM part (green line). The increase in fluorescence intensity is induced by the influx of the PSM-marker Atto488DPPE into the CG-membrane upon lipid mixing between the two membrane systems. By fitting the timeresolved course of the fluorescence intensity with the second order exponential Equation (4-8) (black dashed line), the time constants $t_{1}$ and $t_{2}$ for this two-step process were determined to be $t_{1}=232 \mathrm{~ms}$ and $t_{2}=8.31 \mathrm{~s}$. (B) Boxplots of the obtained half-lives for the two-step process from 50 time-resolved fluorescence intensity traces. For the first, fast increase a mean half-life of $\overline{\boldsymbol{\tau}}_{\mathbf{1} / \mathbf{2}}^{\mathbf{1}}=0.340 \pm 0.335 \mathrm{~s}$ was determined, whereas the mean half-life of the second, slow process resulted in a value of $\overline{\boldsymbol{\tau}}_{\mathbf{1} / \mathbf{2}}^{\mathbf{2}}=8.83 \pm 7.12 \mathrm{~s}$.

The resulting values for $t_{1}$ and $t_{2}$ were multiplied with $\ln (2)$ to obtain the respective half-lives $\tau_{1 / 2}^{1}$ and $\tau_{1 / 2}^{2}$ of the two steps (Figure 4-42B). The mean values of the 50 extracted half-lives for each step were determined to be $\bar{\tau}_{1 / 2}^{1}=0.340 \pm 0.335 \mathrm{~s}$ and $\bar{\tau}_{1 / 2}^{2}=8.83 \pm 7.12 \mathrm{~s}$. The second order exponential Equation (4-8) was not selected arbitrarily, but resulted in the best fit of the data in comparison to first or third order exponential equations of the same type. In Figure 4-43A the exemplary intensity course depicted in Figure 4-42A was fitted with exponential equations with an increasing order of exponential terms from 1 to 3 . The inset shows the reduced $\chi^{2}$ values derived from the different fits, confirming the second order exponential Equation (4-8) to fit the data best. To highlight the two-step mechanism behind this Atto488-DPPE influx into the CG membrane, the parameters $B_{1}$ and $B_{2}$ as well as $t_{1}$ and $t_{2}$ derived from the second order exponential fit function were used to model the intensity trace with two mono exponential functions as depicted in Figure 4-43B. By specifying $y_{0}=0.5$ for the fast process, using $B_{1}$ and $t_{1}$, and $y_{0}=1$ for the slow process, applying $B_{2}$ and $t_{2}$, the two fit functions perfectly cross on the time trace. 
A

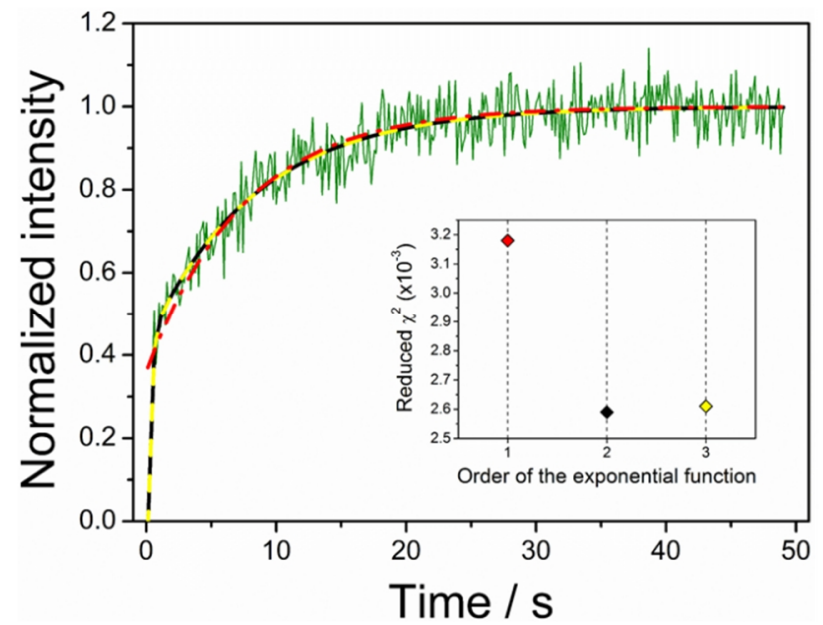

B

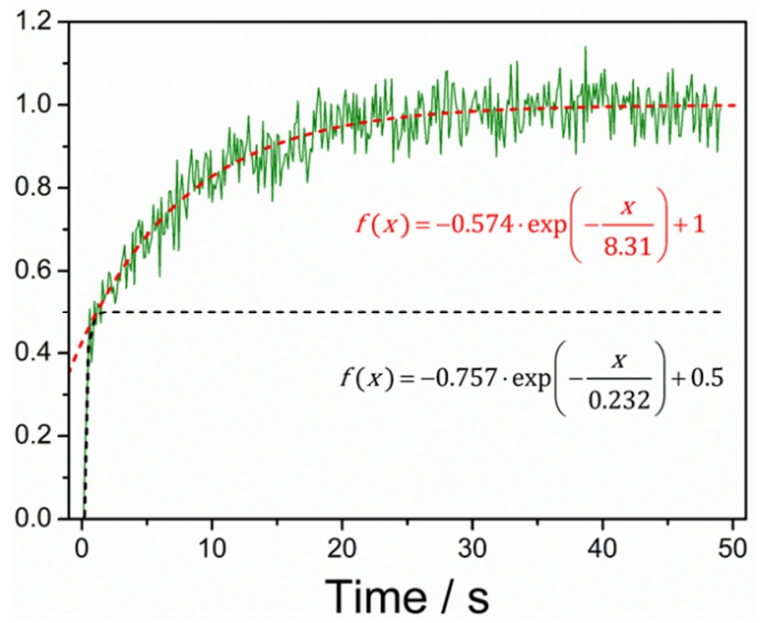

Figure 4-43 (A) Time-resolved fluorescence intensity curve of an Atto488-DPPE influx into the CG membrane fitted with exponential equations of the type of Equation (4-8) with an increasing order of exponential terms ( $1^{\text {st }}$ order: red, $2^{\text {nd }}$ order: black, $3^{\text {rd }}$ order: yellow). The inset depicts the reduced $\chi^{2}$ values derived from the different fits, confirming the $2^{\text {nd }}$ order exponential Equation (4-8) to fit the data best. (B) The same time trace as shown in (A) fitted by two first order exponential functions with parameters derived from the second order exponential fit in (A) specifying $\mathrm{y}_{0}=0.5$ for the fast process (black dashed line) and $\mathrm{y}_{0}=1$ for the slow one (red dashed line). 



\section{Discussion}

The scope of this thesis was to increase the complexity of the assay - introduced by SCHWENEN et al. - for the investigation of SNARE-mediated single vesicle fusion on pore-spanning membranes (PSMs) towards a more physiological model. ${ }^{[44]}$ To this end, two key components of $\mathrm{Ca}^{2+}$-triggered exocytosis, namely $\mathrm{PI}(4,5) \mathrm{P}_{2}$ and synaptotagmin-1 (syt-1) were stepwise integrated into the model system to detect and analyze their impact on SNARE-mediated single vesicle fusion in vitro. Moreover, SNARE-mediated fusion of natural secretory chromaffin granules was analyzed on PSMs on the single particle level to compare their fusion behavior with the characteristics of synthetic single vesicle fusion.

\subsection{Impact of PI $(4,5) P_{2}$ on SNARE-mediated single vesicle fusion}

Biological membranes are frequently found to show significant heterogeneities in their compositions as well as high asymmetries concerning their lipid distribution in the extra- and intracellular leaflet raising the question of whether and how certain lipids affect membrane associated processes. Phosphoinositides, such as $\mathrm{PI}(4,5) \mathrm{P}_{2}$ for example, are found to have a host of crucial functions in plasma membrane associated processes like membrane trafficking, attachment of the cytoskeleton, activation of enzymes as well as in endo- and exocytosis. ${ }^{[47],[133]-[135]}$ With regard to the function in exocytosis, numerous recent studies attribute $\mathrm{PI}(4,5) \mathrm{P}_{2}$ to play an important role in the formation of nano- and mesoscale domains of the t-SNARE syntaxin-1A (syx-1A). ${ }^{[52],[54]}$ Nanodomains of syx-1A with sizes of $\sim 70 \mathrm{~nm}$ in diameter were visualized by high resolution fluorescence microscopy in inverted sheets of PC12 cell-derived plasma membranes and $\mathrm{PI}(4,5) \mathrm{P}_{2}$ was found to be the predominant inner leaflet lipid in these syx-1A domains accumulating to $\sim 82 \%$ of the total surface area. ${ }^{[16],[51]}$ The mechanism of syx-1A sequestering by $\mathrm{PI}(4,5) \mathrm{P}_{2}$ is most probably based on an electrostatic interaction between the juxtamembrane polybasic region (260-KARRKK-265) of the protein and the polyanionic headgroup of the lipid (cf. Chapter 1.3). This property of $\mathrm{PI}(4,5) \mathrm{P}_{2}$ to preorganize t-SNAREs in $\sim 70 \mathrm{~nm}$ sized nanodomains may facilitate SNARE interactions leading to an increase in fusion efficiency. ${ }^{[51],[136]}$ To shed some light on this hypothesis, SNARE-mediated fusion between proteoliposomes and PSMs was investigated on the single particle level with a stepwise increase of the $\mathrm{PI}(4,5) \mathrm{P}_{2}$ content in the t-SNARE containing PSM. 
Indeed, a significantly increased fusion efficiency was observed for SNARE-mediated single vesicle fusion in the presence of $\mathrm{PI}(4,5) \mathrm{P}_{2}$ compared to that in its absence. For proteo-LUVs on PSMs without $\mathrm{PI}(4,5) \mathrm{P}_{2}$ a fusion efficiency of $\sim 50 \%$ was observed, ${ }^{[44],[101]}$ whereas the integration of only $1 \mathrm{~mol} \% \mathrm{PI}(4,5) \mathrm{P}_{2}$ into the lipid composition of the t-SNARE containing $\mathrm{PSM}$ led to an increase of the fusion efficiency to $\sim 79 \%$. A further raise of the $\mathrm{PI}(4,5) \mathrm{P}_{2}$ content in the PSMs to 2 and 5 mol\% resulted in fusion efficiencies for docked proteo-LUVs of $\sim 92 \%$ and $\sim 83 \%$, respectively. It can therefore be concluded that the integration of $\mathrm{PI}(4,5) \mathrm{P}_{2}$ into the lipid composition of the PSM significantly enhanced the fusion efficiency of docked proteoLUVs which may be traced back to the presence of $\mathrm{PI}(4,5) \mathrm{P}_{2}$ induced clustering of the t-SNARE complex $\Delta \mathrm{N} 49$ serving as hot spots for docking and efficient fusion.

But does a PI(4,5) $\mathrm{P}_{2}$ evoked increase in fusion efficiency go hand in hand with an increased fusion kinetics? To answer this question, average lifetimes of the docking state and the intermediate fusion state of single vesicle fusion events on PSMs containing different amounts of $\mathrm{PI}(4,5) \mathrm{P}_{2}$ were analyzed in detail to compare them with the data obtained by SCHWENEN for single vesicle fusion on PSMs without $\mathrm{PI}(4,5) \mathrm{P}_{2} \cdot{ }^{[44],[101]}$ By fitting the kinetic model introduced by FLOYD et al. ${ }^{[122]}$ to the obtained docking time distributions for proteo-LUVs on PSMs with 1,2 and $5 \mathrm{~mol} \% \mathrm{PI}(4,5) \mathrm{P}_{2}$ (cf. Chapter 4.2.4), rate constants for the transition from docking to fusion of $k_{1 \mathrm{~mol} \%}=0.075 \pm 0.003 \mathrm{~s}^{-1}, k_{2 \mathrm{~mol} \%}=0.065 \pm 0.001 \mathrm{~s}^{-1}$ and $k_{5 \mathrm{~mol} \%}=0.084 \pm 0.003 \mathrm{~s}^{-1}$ were achieved corresponding to a number of hidden steps during transition of $N=4$. The reciprocals of the determined rate constants multiplied by the number of steps resulted in typical docking lifetimes on PI(4,5)P2 containing PSMs in the range of 47.6-61.5 s which matches exactly the typical docking lifetime monitored on PSMs without PI(4,5) $\mathrm{P}_{2}$ of $57.6 \mathrm{~s}$. Here, SCHWENEN yielded a lower rate constant of $k_{0 \mathrm{~mol} \%}=0.033 \pm 0.003 \mathrm{~s}^{-1}$ but also a reduced number of hidden steps with $N=1.9 \pm 0.2 .{ }^{[101]}$ The values for the average lifetimes of the docking state were found to be equal in the presence and absence of $\mathrm{PI}(4,5) \mathrm{P}_{2}$, however, the applied kinetic model that was fitted to the distinct docking time distributions definitely demonstrates differences in the underlying mechanism concerning the rate constants and the number of hidden steps. Under the assumption that the number of hidden steps correlates with the number of SNARE complexes that are formed during transition from docking to fusion, the comparison shows that in the presence of $\mathrm{PI}(4,5) \mathrm{P}_{2}(N=4)$ maybe more complexes are involved which work in a cooperative manner as indicated by the higher rate constants. Another explanation for the higher rate constants in the presence of $\mathrm{PI}(4,5) \mathrm{P}_{2}$ could be that even multiple incompletely formed SNARE complexes are sufficient to induce fusion. Concerning the significantly higher fusion efficiency observed in the presence of $\mathrm{PI}(4,5) \mathrm{P}_{2}$, a larger number of 
SNARE complexes at the site of fusion would be a reasonable assumption, yet the average docking lifetime was not found to be reduced in comparison to the absence of $\mathrm{PI}(4,5) \mathrm{P}_{2}$. The reason for this might be the increased negative surface potential of the PSM by introducing the polyanionic phosphoinositide with a negative charge between -3 and -5 under physiological conditions. ${ }^{[137],[138]}$ As the membrane of the proteo-LUV also exhibits a net negative charge (10 mol\% PS), a stronger electrostatic repulsion has to be overcome during spatial approach of the two membranes prior to fusion stalk formation. The assumption that the number of hidden steps might correlate with the number of SNARE complexes formed at the fusion site is with $N=4$ quite reasonable as the estimated number of SNAREs needed to efficiently catalyze fusion was determined in several in vivo and in vitro experiments to range from 3 to $15 .^{[42],[136],[139]-[142]}$

However, the docking lifetimes for SNARE-mediated single vesicle fusion observed in this work are roughly 1000 times slower than those monitored by DOMANSKA et al. for small syb 2 containing vesicles on solid supported membranes (SSMs) with reconstituted $\Delta N 49 .{ }^{[42]}$ The fact that they used exactly the same fusion machinery raises the question what causes this significant difference in the observed docking lifetimes. Besides the different types of target membranes, SSMs vs. PSMs, the two assays significantly differ in the size of syb 2 containing vesicles, the protein to lipid ratio (p/l) used for syb 2 reconstitution as well as in the lipid composition (cf. Table 5-1).

Table 5-1 Comparison of critical parameters of model systems used for the analysis of SNARE-mediated single vesicle fusion.

\begin{tabular}{|c|c|c|c|}
\hline & Model membranes & Initial p/ 1 & Vesicle size \\
\hline \multirow{6}{*}{ This work } & PSMs ( $\triangle \mathrm{N} 49)$ & & \\
\hline & $\mathrm{PC} / \mathrm{PE} / \mathrm{PS} / \mathrm{Chol} / \mathrm{PIP}_{2}$ & $1: 500$ & -- \\
\hline & $(45-49: 20: 10: 20: 1-5)$ & & \\
\hline & LUVs (syb 2); & & \\
\hline & PC/PE/PS/Chol & $1: 500$ & $\varnothing=325 \mathrm{~nm}$ \\
\hline & $(50: 20: 10: 20)$ & & \\
\hline \multirow{4}{*}{ DOMANSKA et al.[42] } & SSMs ( $\Delta \mathrm{N} 49)$ & 1:3000 & -- \\
\hline & PC/Chol (80:20) & & \\
\hline & SUVs (syb 2); & $1: 200$ & $\varnothing=50 \mathrm{~nm}$ \\
\hline & PC/Chol (80:20) & & \\
\hline
\end{tabular}


Comparing only the syb 2 containing vesicles revealed significant differences. The vesicles used in the assay on SSMs were significantly smaller with an average diameter of $50 \mathrm{~nm}$ and contained more than twice as much syb 2. Further, the target membranes and therefore the preparation procedures differ drastically. In our assay, the target membranes - PSMs - were prepared by spreading proteo-GUVs on hydrophilically functionalized porous substrates. With regard to the t-SNARE complex $\triangle \mathrm{N} 49$, we start the reconstitution with a doubled $\mathrm{p} / \mathrm{l}$ ratio. However, it was previously estimated that the reconstitution into GUVs yields a very low efficiency of $\sim 1 \%{ }^{[90]}$ Therefore, the final $\mathrm{p} / 1$ in the PSM would result in $\sim 1: 100.000$ as only half of the reconstituted $\Delta \mathrm{N} 49$ exhibits the right orientation. A more recent and detailed analysis, however, conducted by VouNG concerning the $\triangle \mathrm{N} 49$ reconstitution into GUVs (bachelor thesis in the group of C. STEINEM) revealed a significantly higher mean reconstitution efficiency of $39 \pm 33 \%$ resulting in a mean final $\mathrm{p} / 1$ in the PSM of $\sim 1: 3900$. Yet, the large standard deviation from the mean reflects the strong heterogeneity of the protein to lipid ratio obtained in the proteo-GUVs prepared via electroformation. The target membrane in the assay introduced by DOMANSKA et al. - a solid supported membrane - is prepared by a combined Langmuir-Blodgett/vesicle fusion technique, forming a monolayer in the first step, followed by the spreading of $\Delta \mathrm{N} 49$ containing SUVs on top. There is ample evidence that the efficiency for the reconstitution of $\Delta \mathrm{N} 49$ into SUVs is nearly quantitative, ${ }^{[00],[102]}$ leading to a $\mathrm{p} / \mathrm{l}$ in the SSM of $\sim 1: 3000$. This comparison concerning the $\mathrm{p} / 1$ ratios in the distinct membrane systems shows clear advantages for the assay on SSMs in contrast to our assay, however, there are facts that are still not consistent. DOMANSKA et al., for example, observed a fusion efficiency of only $43 \%$ in their assay, although the SNARE concentrations are supposed to be higher in both membrane systems and the mean vesicle diameter is significantly smaller, resulting in higher curvature stress, known to enhance the fusion efficiency. Another question concerns the formation of a complete SNARE-core complex while using the stabilized $\Delta N 49$ complex as a t-SNARE acceptor complex. Here, it was found that the displacement of the syb 2 fragment (residues 49-96) by the entire SNARE domain of syb 2 (residues 1-96) exhibits a half-life of $19-20 \mathrm{~s}$ as determined by fluorescence anisotropy measurements in bulk solution. ${ }^{[21],[143],[144]}$ As the displacement is prerequisite for the formation of a complete SNARE-core complex, docking lifetimes in the millisecond regime might indicate SNARE specific docking but not a SNARE-induced fusion. This hypothesis is further supported by current studies of KREUTZBERGER et al. in which two different variants of 1:1 (syx-1A/SNAP25) t-SNARE acceptor complexes were introduced and compared with the fusion behavior found on SSMs doped with $\Delta$ N49. ${ }^{[145]}$ They highlighted their results with the finding that the two new $1: 1$ 
complexes showed fusion kinetics/docking lifetimes almost as fast as found for $\Delta \mathrm{N} 49$ and also comparable fusion efficiencies of $\sim 40 \%$. Since in these 1:1 complexes a replacement of the stabilizing fragment is not necessary, the question emerges why SNARE-complex formation and therefore fusion kinetics are not increased but even slightly decreased. The hypothesis that they might observe SNARE specific docking followed by an unknown fusion inducing process is corroborated by the finding of PINCET and coworkers. Their investigation of SNAREcomplex formation by isothermal titration calorimetry revealed that the binding of syb 2's SNARE-domain to the $N$-terminal region of a pre-organized t-SNARE acceptor complex, as it is the case for $\Delta \mathrm{N} 49$, is much faster in comparison to the binding to a $1: 1$ complex of syx-1A and SNAP25. ${ }^{[20]}$ In case of a 1:1 complex, a three helix bundle comprised of two SNARE motifs from SNAP25 and one SNARE motif from syx-1A has to be reorganized prior to the $N$-terminal binding of syb 2's SNARE motif followed by $N$ - to $C$-directional zippering. This would support the theory that a faster $N$-terminal binding of syb 2 containing vesicles to membranes doped with the preorganized $\Delta \mathrm{N} 49$ in contrast to membranes containing 1:1 complexes of syx-1A and SNAP25 would be reasonable but the subsequent zippering process and therefore the formation of the complete SNARE-core has to be significantly faster in case of 1:1 complexes. With regard to the fusion inducing process, many scenarios are conceivable. If one presumes SNARE-specific fusion after an average docking lifetime in the millisecond regime by utilizing $\triangle \mathrm{N} 49$ as the t-SNARE acceptor complex, the only possible explanation would be that a multitude of partially zippered SNARE-core complexes are sufficient to induce fusion what might be enhanced by an adhesive force from the underlying solid support. Indeed, by analyzing the data with different kinetic models, TAMM and coworkers supposed that $7-8$ SNARE complexes are needed to drive fusion. ${ }^{[42],[145]}$ This would additionally explain the lower fusion efficiency of around $40 \%$ that they observed in contrary to the up to $92 \%$ monitored in our assay in the presence of $2 \mathrm{~mol} \% \mathrm{PI}(4,5) \mathrm{P}_{2}$ in the PSM. Further, the protein mobility is drastically reduced in SSMs which may lead to an insufficient number of $\Delta \mathrm{N} 49$ complexes available at the sites of vesicle docking.

As many studies reliably revealed that the energy consuming displacement of the stabilizing fragment in the $\Delta$ N49 complex by the entire SNARE-motif of syb 2 takes place in the order of tens of seconds, ${ }^{[21],[143],[144]}$ the lifetimes of the docking state monitored for single vesicle fusion events in this work appear reasonable assuming SNARE-complex formation between $\triangle \mathrm{N} 49$ and syb 2 to be the main driving force for membrane fusion.

Another characteristic lifetime that was monitored in time traces of single vesicle fusion events on PSMs with different amounts of $\mathrm{PI}(4,5) \mathrm{P}_{2}$ is the lifetime of the intermediate fusion state (cf. 
Figure 4-3). It represents the time interval between the onset of fusion and the onset of collapse of the $\Omega$-shaped post-fusion structure of the proteo-LUV into the PSM. The distributions of the lifetimes of this semi-stable intermediate fusion state showed a single decrease indicating a one-step mechanism. Fitting of a mono exponential decay (Equation (4-3)) to the obtained distributions (cf. Chapter 4.2) resulted in rate constants for the decay of $k_{1 \mathrm{~mol} \%}=0.162 \pm 0.005 \mathrm{~s}^{-1}, k_{2 \mathrm{~mol} \%}=0.147 \pm 0.018 \mathrm{~s}^{-1}$ and $k_{5 \mathrm{~mol} \%}=0.248 \pm 0.014 \mathrm{~s}^{-1}$. As the rate constants for the decay of the intermediate fusion state on PSMs with different $\mathrm{PI}(4,5) \mathrm{P}_{2}$ contents do not vary considerably, it can be concluded that this decay of the semi-stable intermediate fusion state is not significantly dependent on the PI(4,5) $\mathrm{P}_{2}$ content in the PSM. A comparison with the rate constant of decay found by SCHWENEN for proteo-LUVs on PSMs without $\mathrm{PI}(4,5) \mathrm{P}_{2}$ supports this hypothesis. It exhibits a value of $k_{0 \mathrm{~mol} \%}=0.21 \pm 0.02 \mathrm{~s}^{-1}$ which is also similar to the values determined in this work. ${ }^{[44],[101]}$

\subsection{Impact of syt-1 on SNARE-mediated single vesicle fusion}

$\mathrm{Ca}^{2+}$-triggered exocytosis of synaptic vesicles is at the heart of neuronal signal transmission. At present, several proteins and lipids with crucial functions in $\mathrm{Ca}^{2+}$-evoked synchronous neurotransmitter release have been identified. Key players among these are the two anionic phospholipids phosphatidylserine (PS) and phosphatidylinositol-4,5-bisphosphate $\left(\mathrm{PI}(4,5) \mathrm{P}_{2}\right)$ as well as the main candidate for a $\mathrm{Ca}^{2+}$-sensor in neuronal exocytosis - synaptotagmin-1 (syt-1). ${ }^{[66],[146],[147]}$ The mechanism of how syt-1, which is embedded in the synaptic vesicle membrane via a single transmembrane domain, triggers $\mathrm{Ca}^{2+}$-induced exocytosis is debated controversially. Yet, many studies consistently suggest significant interactions between syt-1's $\mathrm{Ca}^{2+}$-binding $\mathrm{C} 2$ domains and the anionic phospholipids PS and $\mathrm{PI}(4,5) \mathrm{P}_{2}$ in the opposing membranes resulting in a $\mathrm{Ca}^{2+}$-independent vesicle tethering followed by a $\mathrm{Ca}^{2+}$-dependent enhanced penetration of the C2 domains into the membranes (cf. Figure 1-10). ${ }^{[16],[103],[143],[147]-}$ ${ }^{[149]}$ In this context, the balance between an inactivating back binding of syt-1 to the host vesicle membrane (cis-interaction) and an activating tethering of two distinct membranes (transinteraction) is supposed to be of major importance. ${ }^{[143],[150]}$ Additionally, recent studies on the fusion behavior of secretory chromaffin granules performed in the JAHN group resulted in the finding that a $\mathrm{Ca}^{2+}$-dependent enhancement was only monitored if the target liposomes contained $\mathrm{PI}(4,5) \mathrm{P}_{2}$ and if polyphosphate anions, such as adenosine triphosphate (ATP) were present. ${ }^{[103]}$ They concluded from these results that polyphosphates might operate by an electrostatic mechanism that reverses the inactivating cis-interaction of syt-1 with its own 
membrane without reducing the activating trans-interaction leading to syt-1 induced tethering ready to react to the $\mathrm{Ca}^{2+}$-trigger.

To illuminate syt-1's mode of action in $\mathrm{Ca}^{2+}$-triggered exocytosis, the model system of porespanning membranes (PSMs) doped with the t-SNARE complex $\triangle N 49$ was utilized in this work to analyze SNARE-mediated single vesicle fusion of proteoliposomes with co-reconstituted syb 2 and syt-1 under different conditions. The lipid mixture of the proteo-LUVs contained $5 \mathrm{~mol} \%$ PS and no PI(4,5) $\mathrm{P}_{2}$, whereas the PSMs contained $10 \mathrm{~mol} \%$ PS and 2 mol\% PI(4,5) $\mathrm{P}_{2}$ (cf. Chapter 4.3.3). The fusion behavior on the single particle level was investigated under three different conditions: firstly, under $\mathrm{Ca}^{2+}-$ and ATP-free conditions $\left(-\mathrm{Ca}^{2+},-\mathrm{ATP}\right)$, referred to as standard conditions, secondly, in the presence of $100 \mu \mathrm{M} \mathrm{Ca}^{2+}$ but without ATP $\left(+\mathrm{Ca}^{2+},-\mathrm{ATP}\right)$ and thirdly, in the presence of both $100 \mu \mathrm{M} \mathrm{Ca}{ }^{2+}$ and $5 \mathrm{mM} \mathrm{ATP}\left(+\mathrm{Ca}^{2+},+\mathrm{ATP}\right)$.

Under standard conditions $\left(-\mathrm{Ca}^{2+},-\mathrm{ATP}\right)$, the fusion efficiency was found to be drastically reduced in the presence of syt-1 (+syt-1: $46 \pm 5 \%)$ in contrast to the fusion efficiency monitored for SNARE-mediated single vesicle fusion in the absence of syt-1 (-syt-1: $92 \pm 4 \%$ ) under equal conditions. The distributions of the observed docking lifetimes, however, were determined to be very similar in both cases ( \pm syt-1) with a maximum of the log-normal distribution (Equation (4-1)) that was fitted to the data at $\tau_{\text {docking }}^{\max }=44 \mathrm{~s}$. However, it should be noted that the broadness of the docking time distribution obtained in the presence of syt-1 with a FWHM of 86 s reflects quite a large inhomogeneity, whereas the FWHM of the docking time distribution in the absence of syt-1 is considerably narrower with 55 s (cf. Figure 4-9A). From these results, it is concluded that syt-1 inhibits SNARE-mediated fusion at least in the absence of $\mathrm{Ca}^{2+}$. This finding is consistent with that of CHAPMAN and coworkers demonstrating that syt-1 probably arrests the SNARE-complex before triggering fast $\mathrm{Ca}^{2+}$-evoked exocytosis. ${ }^{[63]}$ Further, in vivo experiments on synaptic vesicle release in Aplysia neurons showed a significant increase in spontaneous fusion events of 50-75\% while blocking syt-1 expression with antisense oligonucleotides that also provides evidence for syt-1 acting as an inhibitory clamp under $\mathrm{Ca}^{2+}$-free conditions. ${ }^{[151]}$

But how does syt-1 arrest exocytosis in the absence of $\mathrm{Ca}^{2+}$ ? Many studies suggest that syt-1 interacts with both, the SNARE proteins and anionic phospho-lipids in the target membrane in a $\mathrm{Ca}^{2+}$-independent manner. ${ }^{[16],[152]-[157]}$ Therefore, in the absence of $\mathrm{Ca}^{2+}$, syt-1 presumably binds to the target membrane as well as to the SNARE-complex and prevents the complete zippering. Only upon $\mathrm{Ca}^{2+}$-binding, the syt-1/membrane-interaction becomes predominant leading to a deeper penetration of the $\mathrm{C} 2$ domains into the target membrane and, at the same 
time, the syt-1/SNARE-interaction is reduced so that a complete SNARE-complex formation can take place to induce fusion. This hypothesis is corroborated by the finding of PARK et al., who observed that the syt-1/SNARE-interaction is strongly dependent on the ionic strength and can be completely abolished in the presence of polyvalent ions and in particular by pyrophosphates such as ATP. On the contrary, $\mathrm{Ca}^{2+}$ was found to significantly enhance syt-1/membrane-interactions, especially in the presence of $\mathrm{PI}(4,5) \mathrm{P}_{2}$ and $\mathrm{PS}$ in the target membrane. ${ }^{[146]-[148]}$ Thus, the impact of syt-1 on SNARE-mediated single vesicle fusion was analyzed on $\triangle \mathrm{N} 49$ doped PSMs containing $2 \mathrm{~mol} \% \mathrm{PI}(4,5) \mathrm{P}_{2}$ and $10 \mathrm{~mol} \%$ PS in the presence of $100 \mu \mathrm{M} \mathrm{Ca}^{2+}$. Under this condition $\left(+\mathrm{Ca}^{2+},-\mathrm{ATP}\right)$, surprisingly, the fusion efficiency was monitored to be even slightly lower $(38 \pm 4 \%)$ than observed under standard conditions $\left(46 \pm 5 \%\right.$ ). But for those proteo-LUVs that fused with the PSM in the presence of $100 \mu \mathrm{M} \mathrm{Ca}^{2+}$, the docking lifetimes were determined to be significantly shorter with $\tau_{\text {docking }}^{\max }=22 \pm 2 \mathrm{~s}$ $\left(+\mathrm{Ca}^{2+},-\mathrm{ATP}\right)$ in contrast to $\tau_{\text {docking }}^{\max }=44 \pm 9 \mathrm{~s}$ as obtained under standard conditions $\left(-\mathrm{Ca}^{2+}\right.$, -ATP). Moreover, in the presence of $\mathrm{Ca}^{2+}$ the intermediate fusion state was monitored to be nearly non-existent accounting for only $1 \pm 1 \%$ of the observed fusion events. The remaining fusion events $(37 \pm 5 \%)$ were all classified as full fusion events leading to the assumption that a syt-1/Ca ${ }^{2+}$-induced disturbance at the site of fusion promotes a fusion behavior of "all-or-nothing". However, the question remains why the fusion efficiency is slightly lowered in comparison to the standard conditions and what is the reason for the still considerable difference to the fusion efficiency of $92 \pm 4 \%$ monitored in the absence of syt-1. One possible explanation is that part of the detected proteo-LUVs docked to the PSM were adhered by a syt-1/PI(4,5) $\mathrm{P}_{2}$-dominated interaction and not by SNARE complexes. To prove this hypothesis, CLSM measurements were performed to analyze whether docking of syt-1 containing LUVs occurs on PSMs without $\Delta \mathrm{N} 49$ but with $2 \mathrm{~mol} \% \mathrm{PI}(4,5) \mathrm{P}_{2}$ and $10 \mathrm{~mol} \% \mathrm{PS}$ (cf. Chapter 4.3.3). It was found that, even in the absence of $\mathrm{Ca}^{2+}$, proteo-LUVs containing only syt- 1 docked to protein-free PSMs doped with anionic phospholipids supporting the hypothesis that part of the docked proteo-LUVs in the presence of SNARE-proteins are docked to the PSM by a syt-1/membrane-interaction. This assumption would result in a decreased fusion efficiency as the syt-1/membrane-interaction is not able to induce fusion. Another possibility that could be a reason for the reduced fusion efficiency in the presence of syt- 1 is that syt- 1 has to take the right position at the site of fusion to either promote or inhibit fusion. In this context, the balance between the two binding modes cis- (inactivating) and trans- (activating) which are frequently discussed in literature might be important. Here, JAHN and coworkers observed that a $\mathrm{Ca}^{2+}$ enhanced binding of the C2 domains to PS that is localized in the host vesicle membrane (cis- 
interaction) can be reversed in the presence of ATP without affecting the activating transinteraction between the target and the vesicle membrane. ${ }^{[103],[148]}$

To examine whether the balance between cis- and trans-interactions has an influence on SNARE-mediated fusion of syt-1 containing proteo-LUVs on PSMs, measurements were conducted in the presence of both $100 \mu \mathrm{M} \mathrm{Ca}^{2+}$ and $5 \mathrm{mM}$ ATP $\left(+\mathrm{Ca}^{2+},+\mathrm{ATP}\right)$. Under these conditions, a fusion efficiency of $82 \pm 4 \%$ was monitored, which nearly reached the fusion efficiency observed in the absence of syt-1 of $92 \pm 4 \%$. The still slightly lowered fusion efficiency might be caused by proteo-LUVs docked to the PSM via a syt-1/membraneinteraction as already mentioned above. The docking lifetimes in the presence of $\mathrm{Ca}^{2+}$ and ATP were determined to be very similar as observed in the presence of $\mathrm{Ca}^{2+}$ only. However, in the presence of both, $\mathrm{Ca}^{2+}$ and ATP, the distribution of docking lifetimes was found to be narrower providing evidence for a more homogeneous process (cf. Table 5-2). Another observation was that the stalled intermediate fusion state was almost abolished in the presence of $\mathrm{Ca}^{2+}$ and ATP $(1 \pm 1 \%)$ similar to the fraction observed under $\mathrm{Ca}^{2+}$-only conditions. This finding underlines the "all-or-nothing" hypothesis. Further, all detected full fusion events under these conditions $\left(+\mathrm{Ca}^{2+},+\mathrm{ATP}\right)$ were classified as fast full fusion, which is characterized by the decrease of the TxR-DPPE intensity (proteo-LUV marker) to the baseline level within $244 \mathrm{~ms}$ and a collapse of the $\Omega$-shaped post-fusion structure between $0-2$ s (cf. Figure 5-1).
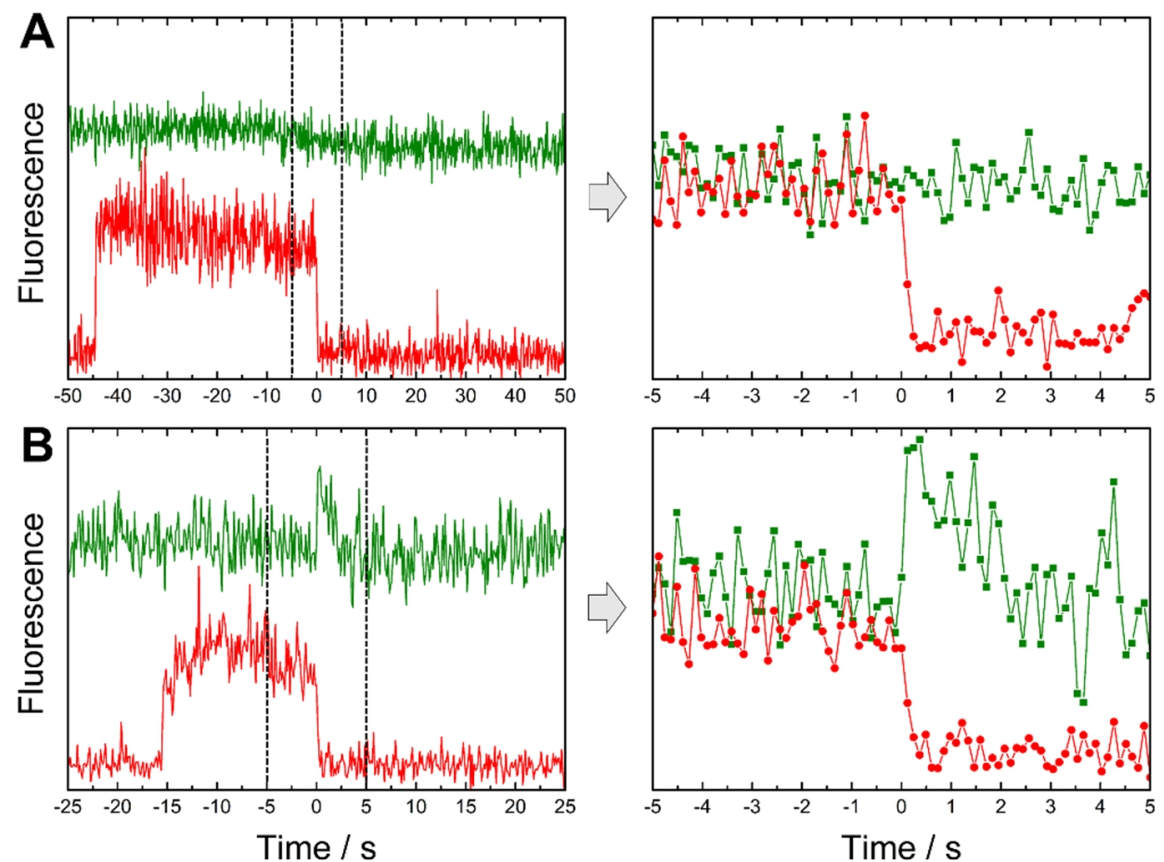

Figure 5-1 Exemplary time-resolved fluorescence intensity traces for fast full fusion events. The red channel corresponds to the TxR-DPPE intensity (proteo-LUV marker) and the green channel to the Atto488DPPE intensity (PSM marker). (A) Fast full fusion event with a decay of the $\Omega$-shaped post-fusion structure that is faster than the time resolution of $122 \mathrm{~ms}$ as no response is observed in the green channel. (B) Fast full fusion event with a collapse of the $\Omega$-shaped post-fusion structure within $\sim 2 \mathrm{~s}$, characterized by the inand decrease in the green channel. 
Unfortunately, the time resolution was not sufficient to analyze the lifetime of the intermediate fusion state in more detail, because it was found to be shorter than $244 \mathrm{~ms}$ ( 2 frames) in most of the full fusion events under these conditions $\left(+\mathrm{Ca}^{2+},+\mathrm{ATP}\right)$. To have a closer look on that, a time-resolution of $\sim 10-20 \mathrm{~ms}$ would be beneficial as achievable by spinning disc fluorescence microscopy.

Table 5-2 Comparison of the proportions of detected events and docking lifetimes monitored for SNAREmediated single vesicle fusion of proteo-LUVs on $\Delta \mathrm{N} 49$ doped PSMs with a PI $(4,5) \mathrm{P}_{2}$ content of $2 \mathrm{~mol} \%$. In the presence of syt-1, co-reconstituted with syb 2 into the proteo-LUVs, the fusion behavior was investigated under three different conditions (1: $-\mathrm{Ca}^{2+}$, -ATP; 2: $+100 \mu \mathrm{M} \mathrm{Ca}{ }^{2+}$, -ATP; 3: $+100 \mu \mathrm{M} \mathrm{Ca}{ }^{2+}$, +5 mM ATP), whereas in the absence of syt- 1 the fusion behavior was analyzed only under standard conditions $\left(-\mathrm{Ca}^{2+},-\mathrm{ATP}\right)$.

\begin{tabular}{|c|c|c|c|c|}
\hline & $\begin{array}{c}\text {-syt-1 } \\
\left(-\mathrm{Ca}^{2+},-\mathrm{ATP}\right)\end{array}$ & $\left(-\mathrm{Ca}^{2+},-\mathrm{ATP}\right)$ & $\begin{array}{c}\text { +syt-1 } \\
\left(+\mathrm{Ca}^{2+},-\mathrm{ATP}\right)\end{array}$ & $\left(+\mathrm{Ca}^{2+},+\mathrm{ATP}\right)$ \\
\hline Docking & $8 \pm 2 \%$ & $54 \pm 6 \%$ & $62 \pm 4 \%$ & $18 \pm 3 \%$ \\
\hline Intermediate fusion & $48 \pm 4 \%$ & $12 \pm 4 \%$ & $1 \pm 1 \%$ & $1 \pm 1 \%$ \\
\hline Full fusion & $44 \pm 4 \%$ & $34 \pm 6 \%$ & $37 \pm 5 \%$ & $81 \pm 4 \%$ \\
\hline$\tau_{\text {docking }}^{\max }$ & $44 \pm 1 \mathrm{~s}$ & $44 \pm 9 s$ & $22 \pm 2 s$ & $25 \pm 1 \mathrm{~s}$ \\
\hline FWHM & $55 \mathrm{~s}$ & $86 \mathrm{~s}$ & $52 \mathrm{~s}$ & $35 \mathrm{~s}$ \\
\hline
\end{tabular}

From the findings made for SNARE-mediated fusion on the single particle level in the absence and presence of the $\mathrm{Ca}^{2+}$-sensor syt-1, a model of different modes of action can be concluded in which syt-1 interacts differently with membranes and SNARE-proteins in response to variation of ionic conditions in the measuring buffer (cf. Figure 5-2). Under standard conditions (20 mM HEPES, 100 mM KCl, 1 mM DTT, 0.1 mM EGTA, pH 7.4), syt-1 was found to inhibit SNARE-mediated fusion. The reason for this might be that syt-1 binds to the t-SNAREs via the polybasic region in the $\mathrm{C} 2 \mathrm{~B}$ domain acting like a fusion clamp that prevents complete zippering of the SNARE-core complex. This fusion inhibiting property of syt-1 was monitored to be partially reversed by adjusting a $\mathrm{Ca}^{2+}$ concentration of $100 \mu \mathrm{M}$. An explanation for this observation might be that $\mathrm{Ca}^{2+}$-binding results in a strongly increased affinity of syt-1's $\mathrm{C} 2$ domains to anionic phospholipids in the target membrane (trans-interaction) and the host vesicle membrane (cis-interaction) leading to a reduced interaction with the SNAREs. ${ }^{[147],[148]}$ This would loosen the clamp on the SNARE-complex and additionally would result in a stronger disturbance of the target membrane, provided that trans-interaction takes place. A cisinteraction, the $\mathrm{Ca}^{2+}$-mediated binding of syt-1's $\mathrm{C} 2$ domains to its host vesicle membrane, however, was determined to be inactivating. ${ }^{[148],[150]}$ Polyvalent anions like ATP were found to 
completely abrogate the electrostatic interaction between syt-1 and the t-SNAREs and beyond that it was observed that also the balance between trans- and cis-interaction is dependent on the presence of polyvalent ions. Here, it was concluded that ATP shifts the balance between transand cis-interaction depending on the kind and amount of anionic lipids in the corresponding membranes. The trans-interaction was found to be favored if the target membrane contains considerable amounts of $\mathrm{PI}(4,5) \mathrm{P}_{2}$ and PS, whereas the host vesicle membrane should contain no $\mathrm{PI}(4,5) \mathrm{P}_{2}$ and no or significantly less $\mathrm{PS} .{ }^{[148],[150]}$ In this work, the syt-1 containing vesicles were prepared without $\mathrm{PI}(4,5) \mathrm{P}_{2}$ and with a PS content of $5 \mathrm{~mol} \%$. The PSMs, on the contrary, contained $2 \mathrm{~mol} \% \mathrm{PI}(4,5) \mathrm{P}_{2}$ and $10 \mathrm{~mol} \%$ PS. Due to the clear difference in the proportion of acidic phospholipids in the respective membranes, the addition of ATP alongside $\mathrm{Ca}^{2+}$ might have shifted all syt-1 interactions in the direction of fusion promoting ones as schematically depicted in Figure 5-2 resulting in the high fusion efficiency of $\sim 82 \%$ and the increased fusion kinetics. Moreover, the $\mathrm{Ca}^{2+}$-promoted insertion of the $\mathrm{C} 2$ domains into the target membrane seems to abolish the intermediate fusion state which was observed to be substantially diminished in the presence of $\mathrm{Ca}^{2+}(\sim 1 \%)$ in comparison to $\mathrm{Ca}^{2+}$-free conditions $(\sim 12 \%)$.

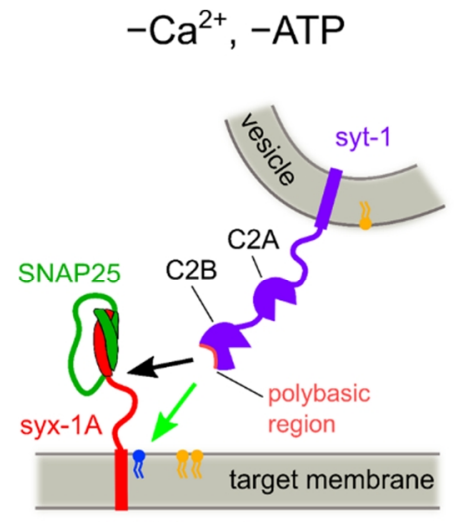

$\mathrm{Pl}(4,5) \mathrm{P}_{2} \mathrm{PS}$
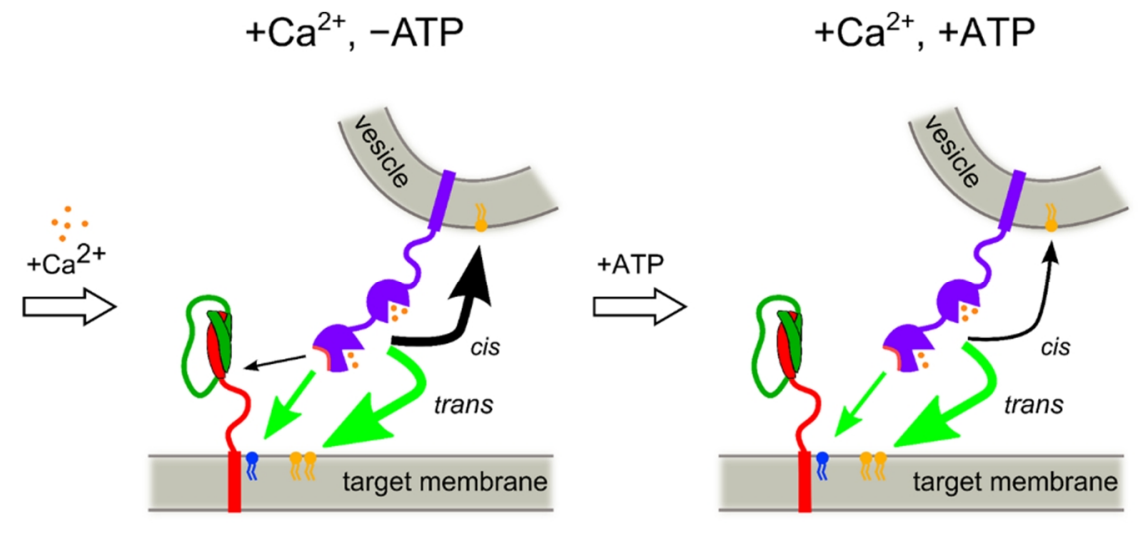

Figure 5-2 Schematic illustration summarizing the interactions of synaptotagmin-1 (syt-1) with t-SNAREs and acidic phospholipid-containing membranes under different ionic conditions as interpreted from the observations made in the context of this work. The green arrows depict fusion promoting interactions, whereas the black arrows represent fusion inhibiting interactions. The widths of the arrows represent the assumed strength of the respective interaction. Under standard conditions $\left(-\mathrm{Ca}^{2+},-\mathrm{ATP}\right), \mathrm{Ca}^{2+}$-independent interactions between the polybasic region and t-SNAREs as well as $\mathrm{PI}(4,5) \mathrm{P}_{2}$ might take place resulting in an overall inhibition of SNARE-mediated fusion. This is partially reversed by introducing $100 \mu \mathrm{MCa}^{2+}\left(+\mathrm{Ca}^{2+}\right.$, -ATP) resulting in a significantly increased affinity of syt-1's C2 domains to anionic phospholipid containing membranes. However, an inactivating cis-interaction as well as a remaining interaction with the t-SNAREs might be present under these conditions still acting partially inhibiting. Only by addition of $5 \mathrm{mM}$ ATP alongside $\mathrm{Ca}^{2+}\left(+\mathrm{Ca}^{2+},+\mathrm{ATP}\right)$, the syt- 1 induced electrostatic interactions might be shifted towards fusion promoting ones by abrogating the syt-1/ SNARE-interaction and enhancing the trans-interaction. 


\subsection{SNARE-mediated fusion of chromaffin granules with pore-spanning membranes}

The regulated exocytosis of secretory granules in neuroendocrine cells such as chromaffin cells is the result of a cascade of events consisting of docking, priming and finally $\mathrm{Ca}^{2+}$-triggered fusion. Several in vivo experiments have shown that the final step of secretion in neuroendocrine cells can occur through different modes of exocytosis classified as "kiss-andrun", "cavicapture" and "full collapse" depending on the physiological demand (cf. Chapter 1.5). ${ }^{[86],[158],[159]}$ The finding that chromaffin granules (CGs) utilize the same set of SNAREs to undergo exocytosis as synaptic vesicles $\mathrm{do}^{[79]}$ as well as the fact that they exhibit similar large diameters in the range of $100-500 \mathrm{~nm},{ }^{[103],[160]}$ makes them a very interesting matter of investigation to monitor the fusion behavior of these natural granules with $\Delta$ N49 doped PSMs on the single particle level and to compare it with the characteristics of synthetic vesicle fusion.

For a fluorescence microscopy based analysis of single CG fusion events on PSMs, the natural secretory granules were isolated from bovine adrenal glands and marked with the fluorescent probe $\mathrm{DiD}-\mathrm{C}_{18}$ - a lipophilic dye that inserts into the outer leaflet of the $\mathrm{CG}$ membrane. Atto488-DPPE labeled PSMs doped with the t-SNARE acceptor complex $\Delta \mathrm{N} 49$ and a PI(4,5) $\mathrm{P}_{2}$ content of $2 \mathrm{~mol} \%$ were prepared as detailed in Chapter 3.2.6. After injecting a suspension of fluorescently labeled CGs above $\triangle$ N49 containing PSM patches, SNARE-specific docking and fusion of these natural secretory granules with PSMs was monitored by confocal laser scanning fluorescence microscopy.

Due to the fact that only the outer leaflet of the CG membrane was fluorescently labeled, a reliable conclusion concerning the appearance and lifetime of an intermediate fusion state, or more precisely a hemifusion state, was not possible. Lifetimes of the docking state, however, were extractable from 104 recorded intensity time traces of single CG fusion events. The evaluation of the docking lifetimes resulted in a very similar distribution (cf. Figure 4-33) in comparison to the one observed for synthetic vesicles containing syb 2 and syt- 1 under the same conditions $\left(-\mathrm{Ca}^{2+},-\mathrm{ATP}\right)$. The log-normal distribution that was fitted to the data led to a maximum at $\tau_{\text {docking }}^{\max }=48 \pm 4 \mathrm{~s}$ and exhibits a FWHM of $85 \mathrm{~s}$. In case of synthetic vesicles doped with syb 2 and syt-1, values for $\tau_{\text {docking }}^{\max }=44 \pm 9$ s and FWHM $=86$ s were obtained. This seems to be a consistent finding, since CGs are not only equipped with the same set of SNAREs as synaptic vesicles but also harbor syt- 1 as a $\mathrm{Ca}^{2+}{ }_{-}$sensor in their membrane. ${ }^{[64]}$ 
Recent studies conducted by TAMM and coworkers, who analyzed SNARE-mediated fusion between PC12-derived dense core vesicles and SSMs, resulted in a mean docking lifetime of $\sim 5 \mathrm{~s}$ before the onset of fusion. ${ }^{[161],[162]}$ In these studies a 1:1 complex of syx-1A and SNAP25 was reconstituted into the SSMs which was attributed to obtain similar fusion kinetics and efficiencies as the $\Delta \mathrm{N} 49$ complex. ${ }^{[145]}$ However, the comparison with this single vesicle fusion assay is limited as firstly: different kinds of dense core vesicles were investigated (the average diameter was not stated), secondly: the preparation and properties of the utilized planar target membranes differ drastically (SSMs vs. PSMs, cf. Chapter 5.1) and thirdly: KREUTZBERGER et al. monitored content release on the single particle level instead of lipid mixing. Since content release requires fusion pore formation, the lifetimes between docking and the onset of lipid mixing might be even shorter in their studies what again raises the question why the docking lifetimes are $10-1000$-fold longer in our assay. The answer to this question remains unclear. However, in comparison to single vesicle fusion observed with our assay, ${ }^{[44],[45],[90],[101]}$ the docking lifetimes monitored for single CG fusion are in good agreement. Moreover, with regard to the observed half-life for the displacement of the stabilizing fragment syb 2 (49-96) in the $\Delta \mathrm{N} 49$ complex by the complete syb 2 SNARE motif of $\sim 20 \mathrm{~s}^{[21],[143],[144]}$, the monitored docking time is in a reasonable range to be the consequence of SNARE complex formation. In addition, SNARE-specific CG fusion was proven by a control experiment in which a blocked t-SNARE complex was reconstituted into the PSM (cf. Chapter 4.5). In this case, neither fusion nor docking of the CGs was determined.

By monitoring lipid mixing between single CGs and PSMs upon SNARE-mediated fusion, three types of CG fusion events were observed which mainly differ in the time when the $\Omega$-shaped post-fusion structure of the CG starts to collapse into the PSM (cf. Figure 4-31). The first type of events (59\%) is characterized by a stable CG post-fusion structure until the end of the time window of observation. A stable $\Omega$-shaped post-fusion structure was interpreted from the Atto488-DPPE influx from the PSM into the CG membrane that reaches a constant saturation level without a subsequent decrease until the end of the recorded time series. The second type of events (34\%) starts similar to the first kind, reaching a saturation level in Atto488-DPPE intensity for a certain time but, in this case, it is followed by a decrease to a random level between the baseline level and the saturation level. A direct collapse was observed for the third type of events ( $7 \%$ ). Here, a sharp increase in Atto488-DPPE intensity is instantly followed by a decrease within $610 \mathrm{~ms}$ (5 frames). Figure 5-3 shows exemplary time-resolved intensity traces of the green channel (Atto488-DPPE intensity) as well as fluorescence 
micrographs of selected frames of the corresponding time series for each type of events. Moreover, line scans were performed to highlight changes in the CG's post-fusion structure.
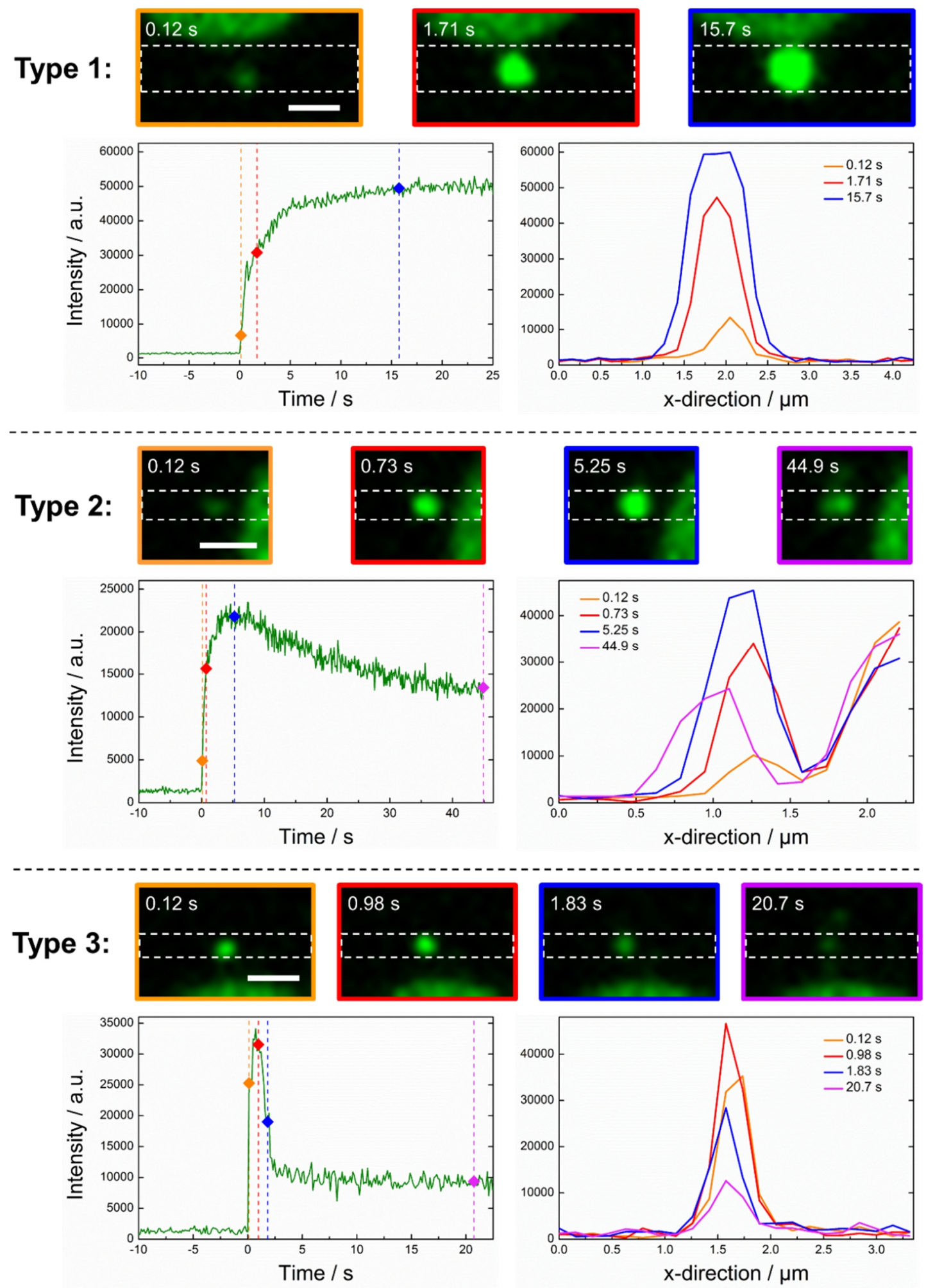

Figure 5-3 Exemplary time-resolved intensity traces of the green channel (Atto488-DPPE intensity, PSM marker) for the three types of single CG fusion events on PSMs. Moreover, fluorescence micrographs of selected frames of the corresponding time series are depicted that were analyzed by line scans to highlight the changes of the $\Omega$-shaped CG post-fusion structure. Type 1 represents a CG fusion event with a stable $\Omega$-profile, whereas in type 2 a delayed shrinking of the $\Omega$-shape occurs and in type 3 a fast collapse. (scale bars: $1 \mu \mathrm{m})$. 
To gain information about the kinetics of the decay of the $\Omega$-shape and to derive the average lifetime of a stable CG post-fusion structure on PSMs, the interval between the onset of fusion and the onset of $\Omega$-collapse $\tau_{\Omega 1}$ (cf. Figure 4-32) was extracted from 98 time traces. The histogram of the binned numbers of $\tau_{\Omega 1}$ (Figure 4-33B) showed a single decrease indicating a one-step mechanism for the collapse of the $\Omega$-shape similar to the observation made for the decay of the intermediate fusion state in synthetic vesicle fusion. By fitting a mono exponential decay (Equation (4-6)) to the data, a rate constant for the decay of $k_{\Omega 1}=0.24 \pm 0.05 \mathrm{~s}^{-1}$ and a corresponding average lifetime of the CG post fusion structure of $\bar{\tau}_{\Omega 1}=4.2 \pm 0.4 \mathrm{~s}$ were determined. The observation of stable or shrinking $\Omega$-shaped post-fusion structures of CGs is in good agreement with recent studies of WU and coworkers who investigated fusion generated $\Omega$-profiles of single CGs in live chromaffin cells by means of STED-microscopy. ${ }^{[163],[164]}$ From these studies, a highly dynamic model for changes of the CG post-fusion structure was concluded referred to as $\Omega$-exo-endocytosis. Their model suggests to redefine the fusion modes of "kiss-and-run" and "full collapse" as rapid or slow closures of the $\Omega$-shaped profile, during which this profile can undergo changes in shape, resulting in various sizes of post-fusion structures (cf. Figure 5-4 left).

Wu \& coworkers:

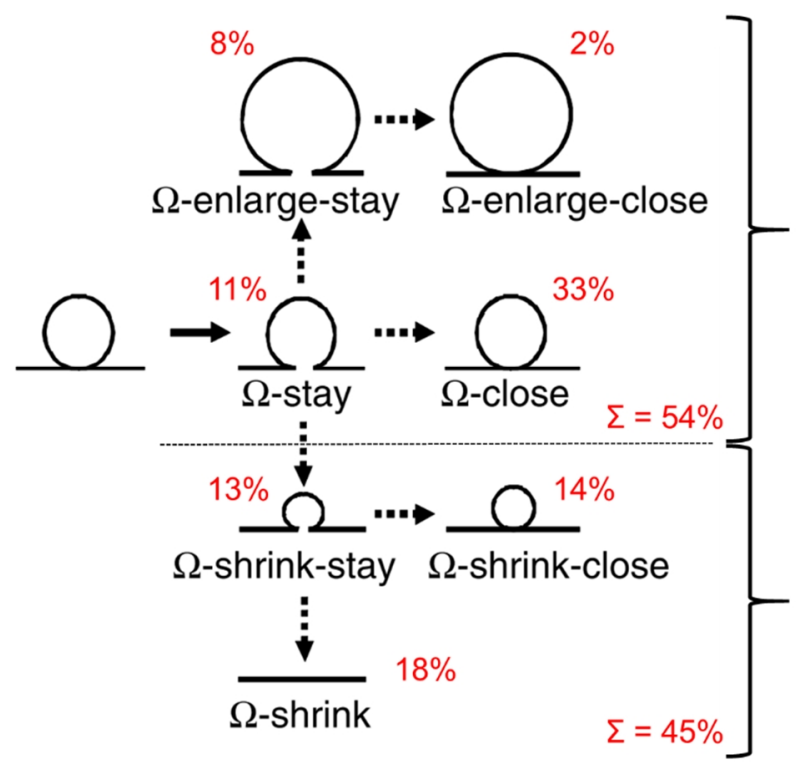

This work:

Type 1:

$(59 \%)$

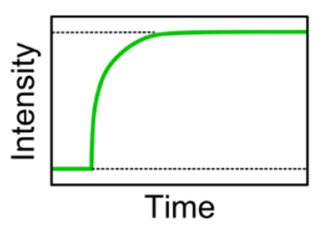

Type 2:

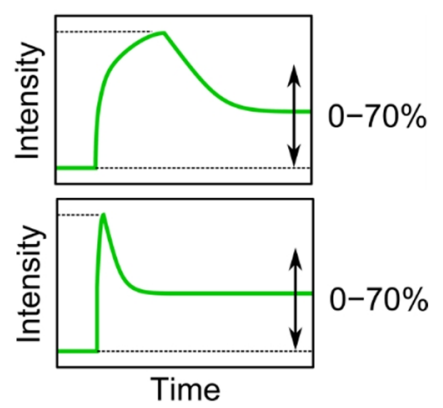

Figure 5-4 Comparison of single CG fusion events with regard to changes in the $\Omega$-shaped post-fusion structure. (left) Schematic drawing of the $\Omega$-exo-endocytosis model introduced by WU and coworkers.[163] They monitored content in- and outflux of single CGs as well as the distribution of fluorescently labeled syb 2 from the CG into the plasma membrane in live chromaffin cells by STED-microscopy. The observed single CG fusion events were classified into the 7 modes that are depicted with the respective percentages (red). (right) Three types of single CG fusion events observed on PSMs in the context of this work by monitoring lipid mixing. The schematic drawings illustrate the Atto488-DPPE influx ( green) from the PSM into a CG with a stable $\Omega$-shaped post-fusion structure (Type 1), with a semi-stable post-fusion structure that starts to shrink after a certain time (Type 2) or with an unstable $\Omega$-shape that collapses immediately after the onset of fusion (Type 3). 
By monitoring content in- and outflux as well as the distribution of fluorescently labeled syb 2 from the CG into the plasma membrane upon fusion in live chromaffin cells, WU and coworkers were able to classify single CG fusion events into 7 different modes that are depicted in Figure 5-4 on the left. Their findings clearly demonstrate that CG post-fusion structures can undergo a great variety of changes in their $\Omega$-profile from enlargement through staying constant to shrinking and ultimately complete collapse which can be related to the observations for in vitro single CG fusion made in this work. Since only lipid mixing between single CGs and PSMs was monitored, the classification into three types was not as detailed, but the determined percentages for the three types are similar to the sum of percentages of the corresponding modes detected by CHIANG et al. (cf. Figure 5-4). However, a complete collapse of CG post-fusion structures on PSMs was only observed in very rare cases (4 events $\hat{=} 1 \%)$ in the free-standing area (f-PSM) or at the pore edge. The reason for this might be the dense core vesicle content mainly comprised of neuroactive peptides and a multitude of proteins. A translocation of this content after full collapse of a CG on the s-PSM might be very slow in the thin water layer between the hydrophilically functionalized surface and the lipid bilayer. For synthetic vesicles on PSMs, a full collapse of the $\Omega$-shaped post-fusion structure was monitored in a significantly higher percentage (30-80\%, depending on the conditions) as they are filled with buffer solution instead of proteins and peptides.

Another striking difference between the fusion behavior of natural CGs on PSMs and that of synthetic vesicles was identified in the time traces of the green channel, representing the influx of Atto488-DPPE from the PSM into the vesicle or rather CG membrane. In case of synthetic vesicle fusion, as long as remaining in the intermediate fusion state, the time-resolved increase in the green channel was found to be fitted best by a first order exponential equation (cf. Chapter 4.4.1). The analysis of 22 time traces yielded a mean half-life for the diffusion of Atto488-DPPE from the PSM into the synthetic vesicle membrane of $\bar{\tau}_{1 / 2}^{1}=0.121 \pm 0.065 \mathrm{~s}$. For single CG fusion, however, as long as a stable $\Omega$-shape existed, the time-dependent increase in Atto488-DPPE intensity was modeled best by a second order exponential equation (cf. Chapter 4.5.2) indicating a two-step process with a fast and a slow time constant. The evaluation of 50 time traces resulted in a mean half-life for the first, fast process of $\bar{\tau}_{1 / 2}^{1}=0.340 \pm 0.335 \mathrm{~s}$ and for the second, slow process of $\bar{\tau}_{1 / 2}^{2}=8.83 \pm 7.12 \mathrm{~s}$. The large standard deviations in case of CG fusion clearly demonstrate a more pronounced inhomogeneity in the fusion process in comparison to synthetic vesicle fusion. Boxplots of the determined half-lives for both, CG and synthetic vesicle fusion are shown in Figure 5-5B. Moreover, exemplary Atto488-DPPE intensity time traces for a synthetic vesicle fusion event stalled in the intermediate fusion state 
and for a CG fusion event with a stable $\Omega$-shape are depicted. This direct comparison of timedependent intensity courses of the Atto488-DPPE influx into the respective membrane - natural CG vs. synthetic vesicle - unambiguously reveals the different nature of this process for the two distinct fusion systems.

A

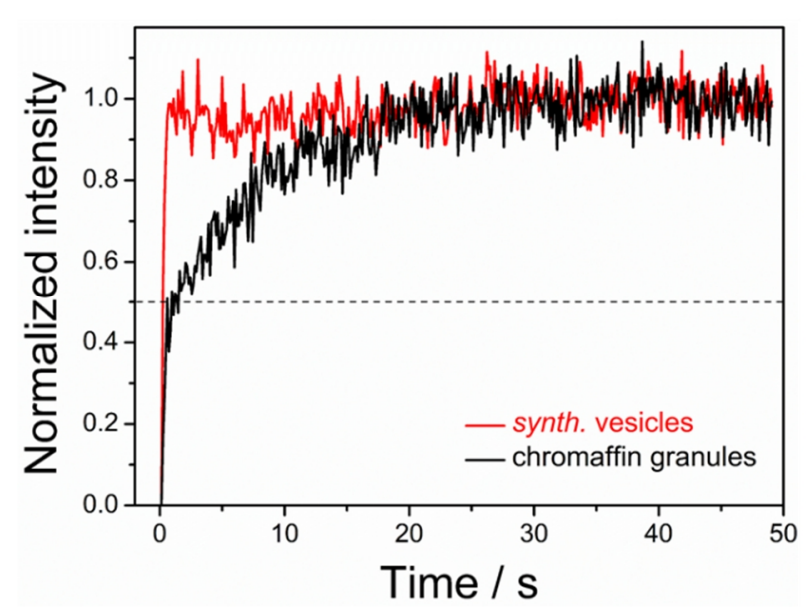

B

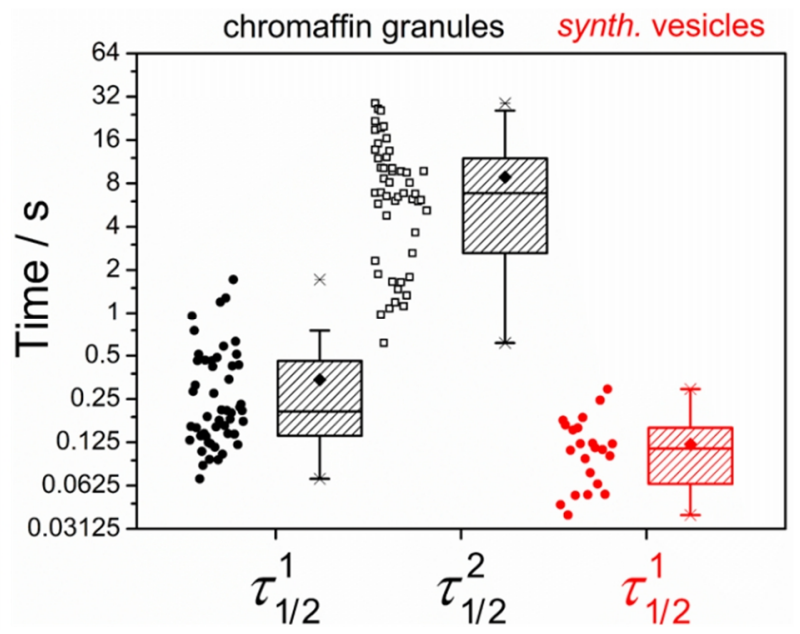

Figure 5-5 (A) Comparison of normalized, time-resolved fluorescence intensity traces of a CG fusion event (black) with a stable $\Omega$-shape and a synthetic vesicle fusion event (red) stalled in the intermediate fusion state. The intensity courses result from an Atto488-DPPE influx from the PSM into the respective membrane. The CG time traces indicate a two-step process, whereas the synthetic vesicle time traces show a one-step behavior. (B) Boxplots of half-lives for the two-step process of CGs (black; $n=50$ ) and the onestep process observed for synthetic vesicles (red; $n=22$ ). The time constants were derived by fitting a first order exponential equation (Equation (4-5)) to the synthetic vesicle data and a second order exponential equation (Equation (4-8)) to the CG data. For the two-step process of the CGs, mean half-lives of $\bar{\tau}_{1 / 2}^{1}=0.340 \pm 0.335 \mathrm{~s}$ and $\bar{\tau}_{1 / 2}^{2}=8.83 \pm 7.12 \mathrm{~s}$ were obtained, whereas the half-life for the one-step process of synthetic vesicle fusion resulted in a mean of $\overline{\boldsymbol{\tau}}_{\mathbf{1 / 2}}^{\mathbf{1}}=0.121 \pm 0.065 \mathrm{~s}$.

What could be the reason for the different diffusion behavior of Atto488-DPPE into the distinct membrane systems? One explanation could be that the Atto488-DPPE diffusion is more hindered in the densely crowded CG membrane in comparison to that of synthetic vesicles. For that scenario, however, a one-step process for the Atto488-DPPE influx into the CG membrane with a lager half-life would be explored. Since this is not the case, another explanation is required that includes the two-step behavior found for CG fusion. Many indications suggest a hemifusion intermediate followed by a flickering pore to induce such an influx behavior. The fast first increase to $\sim 50 \%$ of the final intensity would reflect the formation of a hemifusion intermediate, leading to a saturation of the outer CG leaflet with Atto488-DPPE within several hundreds of milliseconds. This hypothesis is supported by the finding that the half-life of the Atto488-DPPE influx into synthetic vesicles, stalled in the intermediate fusion state, is in a comparable time range as the fast process of CG fusion. The shift to a $\sim 3$ times larger mean half-life for the fast process in case of CG fusion might be caused by a narrower hemifusion 
stalk or the densely crowded CG membrane that may impede the Atto488-DPPE influx. In a recent study of ZHAO et al., where high resolution STED microscopy was applied to analyze cross sections of single CG $\Omega$-profiles in live chromaffin cells, $\Omega$-structures were resolved that exhibit characteristics of a hemifusion intermediate on the pathway to fusion pore formation. They corroborated their findings by electron microscopy derived tomographs of presumable hemifusion structures. ${ }^{[164]}$

Instantly after the formation of a hemifusion intermediate, the distal leaflets of the CG and the PSM might form a flickering pore by which Atto488-DPPE diffusion into the inner leaflet of the CG is significantly slowed down resulting in the second process with the larger half-life. The occurrence of flickering pores in combination with the "kiss-and-run" mode of exocytosis is often used to interpret CG secretion behavior monitored in vitro. ${ }^{[76],[84],[165],[166]}$ CABEZA et $a l$. , for example, studied endo- and exocytosis of secretory granules in live mast cells with different patch clamp techniques and observed transient capacitance flickers with an average half-life of $5.6 \mathrm{~s} .{ }^{[167]}$ They concluded from their observation that these flickers might be induced by the flickering of a dilated fusion pore.

Beyond this very complex declaration of the origin of the two-step mechanism including a hemifusion intermediate and a subsequent flickering pore, another very simple explanation is left. As mentioned above, WU and coworkers impressively proved that $\Omega$-profiles of CG postfusion structures are very dynamic structures that can undergo drastic changes in size. ${ }^{[163]}$ So, the monitored two-step mechanism might be caused by a first, fast opening of the fusion pore, allowing Atto488-DPPE to diffuse into the CG membrane, followed by a second, slow enlargement of the CG post-fusion structure leading to a growing distance to the underlying, fluorescence quenching gold layer. This enlargement can be induced by buffer influx through a slowly dilating fusion pore to balance the osmotic difference originating from the dense core granule nature of a highly concentrated content. Nevertheless, to provide a more reliable statement for the origin of the two-step process, single CG fusion needs to be analyzed by monitoring both content mixing and lipid mixing. In that case, hemifusion could unambiguously be discriminated from other intermediate fusion states evoked by pore opening and closure steps. With regard to the system of PSMs, an elegant variant would be to enclose a water soluble dye in cavities sealed by a cavity-spanning lipid bilayer and to label a membrane associated protein in the CG with a fluorescent antibody. By applying such a strategy, fusion pore formation can be monitored by influx of the fluorescent dye into the granule lumen. Further, the distribution of the labeled granule membrane associated protein upon collapse of 
the CG post-fusion structure into the PSM might provide interesting insights about the fate of the $\mathrm{CG}$ membrane.

\subsubsection{Diffusion behavior of chromaffin granules on PSMs}

For the investigation of SNARE-mediated single CG fusion in vitro, pore-spanning membranes (PSMs) doped with the t-SNARE acceptor complex $\triangle \mathrm{N} 49$ (cf. Chapter 3.2.6) were utilized as a model system to mimic the plasma membrane of a chromaffin cell. PSMs are comprised of a free-standing lipid bilayer part (f-PSM) and a solid supported one (s-PSM) as shown in Figure 4-2. During the investigation of SNARE-mediated single vesicle fusion on PSMs, KUHLMANN observed that synthetic vesicles, docked to the f-PSM, were fully mobile with a mean diffusion coefficient of $0.42 \pm 0.15 \mu \mathrm{m}^{2} / \mathrm{s}$, while docking to the s-PSM resulted in an immediate immobility of the vesicle. ${ }^{[45],[90]}$ The same diffusion behavior of synthetic vesicles on PSMs was monitored in the context of this work but since the focus was on the impact of $\mathrm{PI}(4,5) \mathrm{P}_{2}$ and syt-1 on SNARE-mediated single vesicle fusion, the diffusion behavior was not described in here. For single CG fusion on PSMs, however, the diffusion behavior was analyzed in detail (cf. Chapter 4.5.1). In contrast to synthetic vesicles, SNARE-bound CGs were found to be mobile on both parts of the PSM. However, the mean diffusion coefficient of a CG docked to the s-PSM was determined to be about 3 times lower $\left(0.12 \pm 0.05 \mu \mathrm{m}^{2} / \mathrm{s}\right)$ in comparison to a CG docked to the f-PSM $\left(0.34 \pm 0.06 \mu \mathrm{m}^{2} / \mathrm{s}\right)$. This difference in diffusivity by the factor of $\sim 3$ was also found for the diffusion of a fluorescently labeled syx-1A transmembrane domain (TMD) in the two distinct parts of PSMs. In this case, mean diffusion coefficients for the syx-1A TMD of $1.0 \pm 0.3 \mu \mathrm{m}^{2} / \mathrm{s}^{[90]}$ in the s-PSM and $3.4 \pm 0.2^{[44]}$ in the f-PSM were determined. This reduction in mobility on the s-PSM in both cases might be caused by a frictional coupling to the solid support or by an interaction with the hydrophilic, self-assembled monolayer (SAM) of 6-mercaptohexan-1-ol (6-MH) on the gold surface, as the syx-1A TMD, which also anchors the CGs in the PSM, penetrates both leaflets.

In a recent study by SPINDLER, the diffusion behavior of single gold nanoparticle (GNP) labeled lipids on PSMs was analyzed by means of interferometric scattering microscopy (iSCAT). Interestingly, it was found that the mean diffusion coefficients of these GNP labeled lipids do not differ on the distinct parts of the PSM, at least when the same functionalization of the solid support was applied as in this work (gold layer $\rightarrow 6-\mathrm{MH}$ SAM). For lipids labeled with $40 \mathrm{~nm}$ GNPs, mean diffusion coefficients of $2.0 \pm 0.6 \mu \mathrm{m}^{2} / \mathrm{s}$ in the s-PSM and $1.8 \pm 0.6 \mu \mathrm{m}^{2} / \mathrm{s}$ in the f-PSM were determined. ${ }^{[168]}$ This observation, however, does not contradict the finding of a 
reduced CG diffusion on the s-PSM made in this work, since the GNP labeled lipid is only embedded in the upper leaflet of the PSM which diffusivity is, as indicated by the results of SPINDLER, not considerably influenced by the properties of the underlying leaflet. This finding, however, was unique for PSMs prepared on the above mentioned gold $\rightarrow$ 6-MH surface functionalization of the solid support. If preparing PSMs directly on an oxygen plasma cleaned silicon nitride surface, significantly different mean diffusion coefficients were determined for GNP labeled lipids in the distinct parts of the PSM. In this case, a mean diffusion coefficient of $0.69 \pm 0.03 \mu \mathrm{m}^{2} / \mathrm{s}$ was monitored in the s-PSM, whereas a considerably higher mean diffusion coefficient of $2.65 \pm 0.33 \mu \mathrm{m}^{2} / \mathrm{s}$ was observed in the f-PSM indicating a strong membrane adhesion of s-PSMs directly applied on $\mathrm{S} \mathrm{SiO}_{\mathrm{x}}$ surface. This result is in line with the observation made by TAMM and coworkers who monitored immediate immobility of CGs docked to a tSNARE doped SSM. ${ }^{[161],[162]}$ Since CG mobility was found in various in vivo studies to be an important characteristic to draw conclusions on their fusion state (docked, primed) or the fusion mode ("kiss-and-run", full collapse), ${ }^{[80],[129],[130],[132],[169]}$ the model system of PSMs on a hydrophilic, 6-MH-SAM-functionalized surface exhibits a valuable approach to analyse CG fusion in vitro.

For CGs docked to PSMs, different kinds of diffusion were monitored, ranging from a confined diffusion in a single pore (rare cases) (cf. Figure 5-6A) to a free diffusion on the rim and across the pore boundaries (most cases) (cf. Figure 5-6B). Some CG diffusion trajectories even showed a directed diffusion behavior in the time window of observation (cf. Figure 5-6C). In most cases, a free diffusion of docked CGs on both parts of the PSM was observed with the ability to cross the pore boundaries (cf. Figure 4-35). However, the analysis of the probability density (PD) of CG locations clearly demonstrated a preference of the diffusing CGs to stay on the s-PSM (PDs-PSM $\sim 80 \%$ ) (cf. Figure 4-40 and Table 4-8). This preference might have resulted in some cases in a directed diffusion and might be caused by an adhesive force originating from the solid support.

Prior to fusion, indicated by lipid mixing between a CG and the PSM, the diffusing CGs had been stuck for at least $2 \mathrm{~s}$ ( $\hat{=} 16$ frames) at the site of fusion before lipid mixing started. In some cases, CGs even remained stuck for more than $60 \mathrm{~s}$ before the onset of fusion or did not fuse until the end of the recorded time series. Another diffusion behavior monitored for CGs in the docking state was that they frequently got stuck at certain positions on the s-PSM or at the pore edge, stayed there or restarted moving after varying times. Surprisingly, a similar behavior was observed by SPINDLER for GNP labeled lipids on PSMs despite the fact that the lipids were only embedded in the upper leaflet. ${ }^{[168]}$ The observed transient immobilization might be caused by 
defects on the support or in the lipid bilayer. For CG diffusion, however, immobile t-SNARE cluster on the s-PSM would also be a reasonable assumption to induce such pinning points. In the f-PSM, CG immobilization was only observed at the pore edge, underlining the significant impact of the solid support in this process.
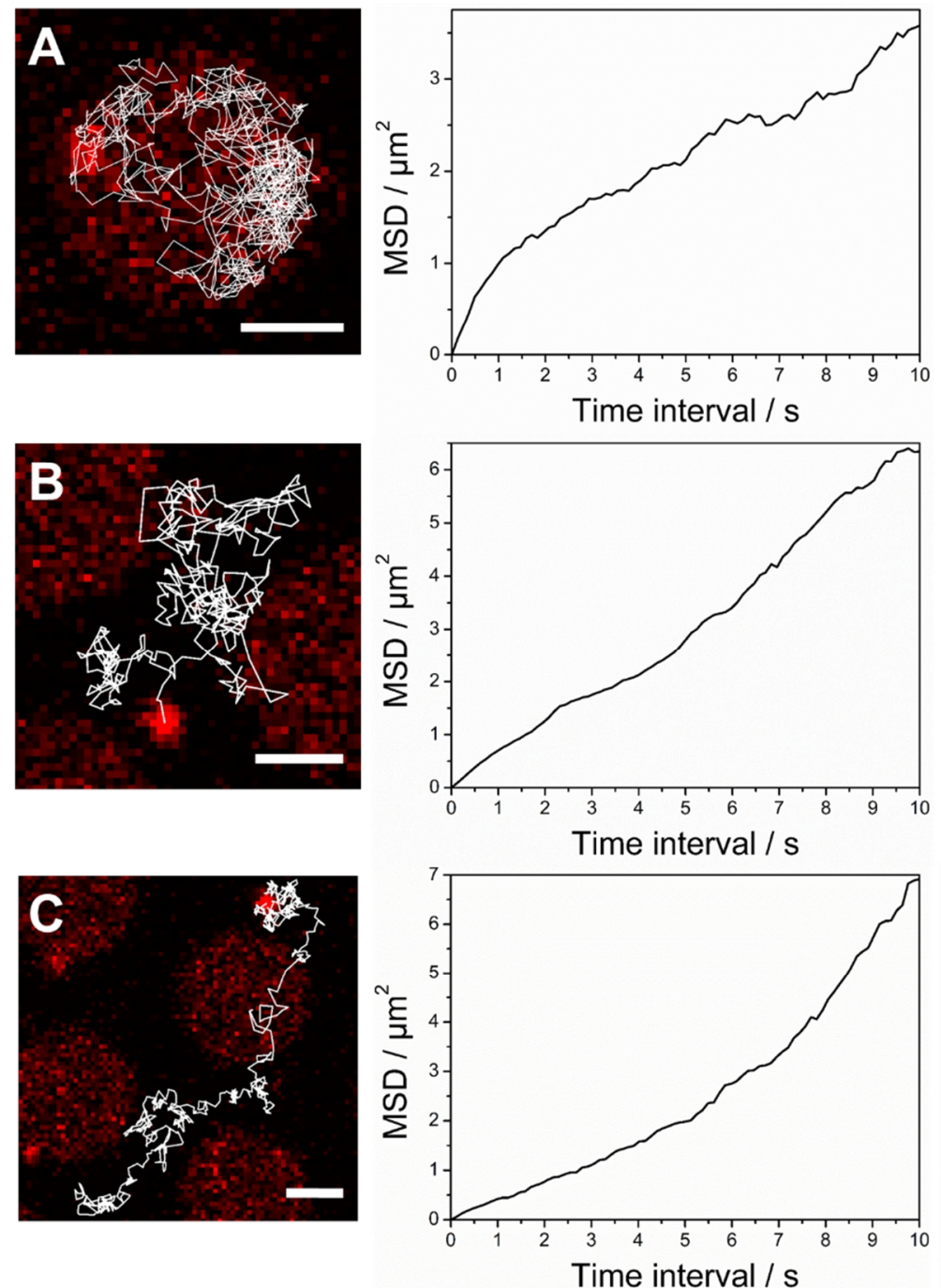
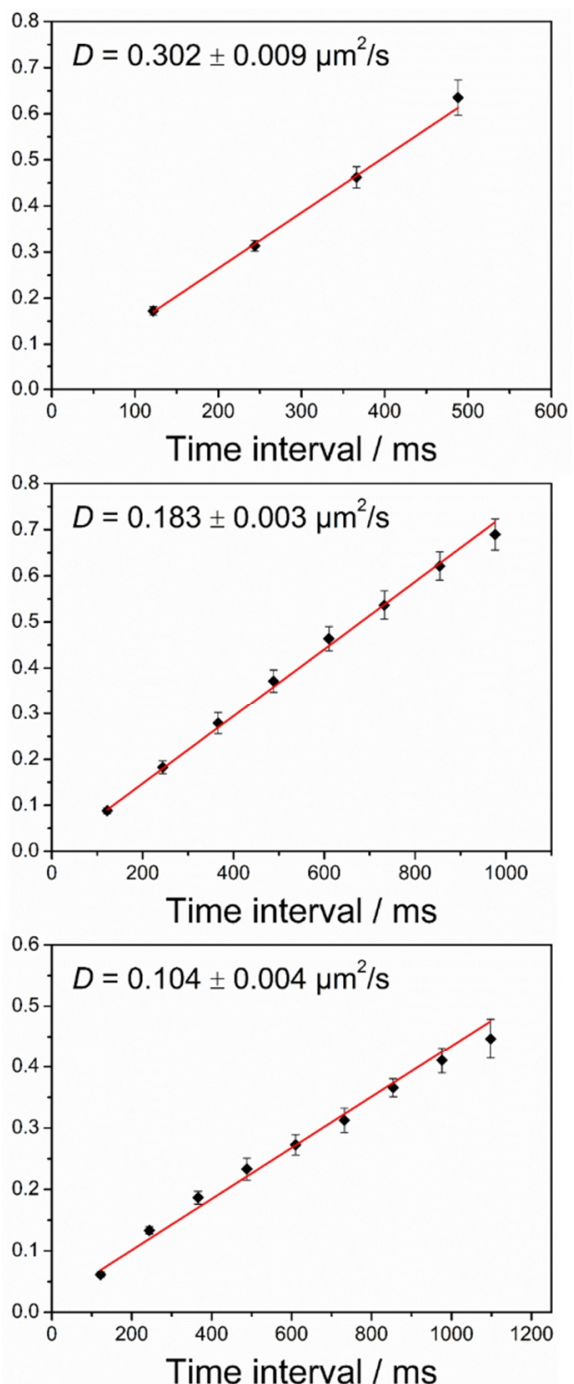

Figure 5-6 (left) Fluorescence micrographs of exemplary diffusion trajectories (white lines) of CGs (red) docked to PSMs representing a confined diffusion (A), a free diffusion (B) and a directed diffusion (C). (scale bars: $2 \mu \mathrm{m}$ ) A confined diffusion occurs if the CG cannot cross the pore boundary. As a result, the plot of the mean square displacement (MSD) against increasing time intervals of up to $10 \mathrm{~s}$ (middle) shows a negative curvature. The diffusion coefficient was obtained from the slope (4D) of a MSD plot against short time intervals (right). For free diffusion, representing a random walker, the MSD is a linear function of time intervals and in case of a directed diffusion, the MSD vs. time interval plot shows a positive curvature.

Interestingly, NOFAL et al. observed a very similar diffusion behavior of single CGs in live chromaffin cells as monitored for single CGs on PSMs. ${ }^{[169]}$ In their study, CGs were stained by overexpressing neuropeptide Y (NPY) fused to monomeric red fluorescent protein (mRFP) and their mobility in live chromaffin cells was visualized by total internal reflection fluorescence microscopy (TIRFM). With this method, they were able to identify three different types of CG 
mobility - namely immobility, restricted caged mobility and free or directed mobility. They referred these different types of motion to the distinct pools of primed CGs, docked CGs and CGs in depot, respectively. Further, an evaluation strategy called caging diameter (CD) was applied to each $\mathrm{CG}$ diffusion trajectory which allowed a quantitative measure of the dynamic changes of mobility. The CD was defined as the maximal distance a CG travels within a fixed time window of $6 \mathrm{~s}$. Figure 5-7 summarizes the essential observations made by NoFAL et al. for CG diffusion in live chromaffin cells.

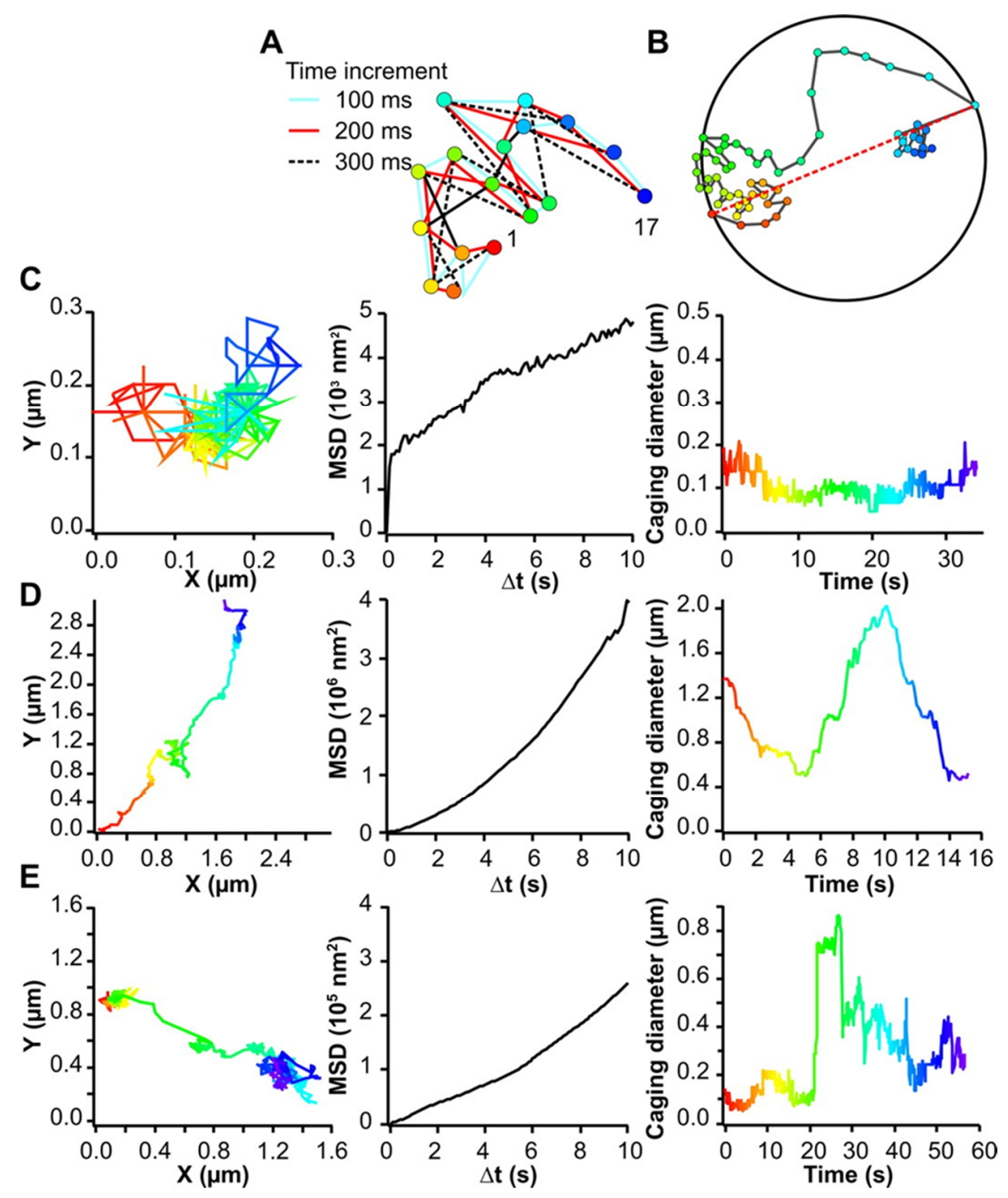

Figure 5-7 This graphic was derived from NoFAL et al.[169] and summarizes their observations made for CG diffusion in live chromaffin cells. (A+B) Schematic illustrations to highlight the difference in the mobility evaluation methods of MSD vs. $\Delta t(\mathrm{~A})$ and CD vs. $\mathrm{t}(\mathrm{B})$. In contrast to the MSD vs. $\Delta \mathrm{t}$ method, which reflects an average diffusion behavior, the $\mathrm{CD}$ vs. t method allows to identify changes in mobility over time. (C-E) Exemplary CG diffusion trajectories with distinct types of motion monitored in live chromaffin cells. The middle represents the MSD vs. $\Delta t$ analysis of the corresponding trajectory on the left and on the right the result of the CD vs. $t$ analysis is depicted. In case of a restricted caged CG diffusion (C), the MSD vs. $\Delta t$ plot shows a negative curvature and the caging diameter remains very small over time. The CG diffusion trajectory depicted in (D) represents an active directed motion which is characterized by a positive curvature in the MSD vs. $\Delta t$ plot and also by strong fluctuations in the CD vs. t. The MSD vs. $\Delta t$ plot for the CG diffusion trajectory depicted in (E) indicates on average a constant and free diffusion while the CD vs. $t$ analysis describes the diffusion behavior more detailed, subdividing the CG motion into an immobile start $(0-20 \mathrm{~s})$ followed by a mobile section ( 20-30 s) and finally a reduced mobility. 
Another in vivo study performed by HENRY and coworkers focused on the analysis of secretory vesicle motion in human carcinoid BON cells. ${ }^{[131]}$ Also for this different kind of secretory vesicles and cell line, a very similar diffusion behavior was observed by TIRFM. The monitored diffusion trajectories of the secretory vesicles again showed a very transient behavior and were subdivided into the motion states of stalled, constrained, free and directed diffusion. ${ }^{[131]}$ Apart from the strong similarities observed in vivo in comparison to the diffusion behavior monitored for CGs on PSMs, the determined diffusion coefficients in vivo were found to be significantly lower by an order of magnitude $\left.\left((2.07 \pm 0.06) \cdot 10^{-3} \mu \mathrm{m}^{2} / \mathrm{s}^{[170]},(2.2 \pm 0.8) \cdot 10^{-3} \mu \mathrm{m}^{2} / \mathrm{s}^{[131]}\right)\right)$. The reason for this might be a higher viscosity within the cytosol as well as the fact that the cells were plated on glass cover slips which most probably results in a reduced mobility of the SNAREs and other membrane component in the plasma membrane.

In the afore-referenced in vivo studies ${ }^{[131],[169]}$, secretory vesicle diffusion was characterized only prior to fusion owing to the fact that they were based on content labeling lacking a membrane marker. The analysis of CG diffusion on PSMs, however, was also conducted after fusion. The onset of fusion was indicated by lipid mixing between the two opposing membranes resulting in an Atto488-DPPE influx from the PSM into the CG membrane and an outflux of DiD- $\mathrm{C}_{18}$ from the CG membrane into the PSM. As mentioned before, the CGs were monitored to immobilize at least $2 \mathrm{~s}$ prior to the onset of fusion. As a result, most of the CGs on the s-PSM remained in the immobile state after the onset of fusion. However, in rare cases, CGs on the sPSM were observed to loosen from their fusion site and start to diffuse again (cf. Figure 5-8B). Nevertheless, the determined mobilities on the s-PSM of CGs post-lipid-mixing were observed to be 3 times lower than for CG diffusion pre-lipid-mixing (cf. Chapter 4.5.1). This might be a result of a larger contact area between the CG and the PSM after fusion inducing a stronger frictional coupling and adhesion to the solid support. For CG diffusion on the f-PSM, however, similar mean diffusion coefficients were determined for both the pre- and post-lipid-mixing states (cf. Figure 4-34). Although the average mobility was found to be equal on the f-PSM, a significant difference in the diffusion behavior was observed regarding the ability to cross the pore boundary. Interestingly, for CGs in the docking state the pore edge was not observed to be a significant barrier. Taking the CG location analysis into account (cf. Figure 4-40), it seems as if docked CGs, especially in the lower mobility state $\left(D<0.05 \mu \mathrm{m}^{2} / \mathrm{s}\right)$, tend to avoid to enter the pore. This corroborates the hypothesis of an adhesive force originating from the solid support as exiting the pore was monitored to be an almost unhindered transition for docked CGs. This was found to be different for CG diffusion post-lipid-mixing. In cases of CG fusion sites at the edge of a pore or in the vicinity of a pore on the s-PSM, the CG post-fusion structure 
was sometimes monitored to enter the pore in which it was fully mobile. To exit the pore again, however, was observed to be rare resulting in a confined diffusion of the CG post-fusion structure as shown in Figure 5-8A.
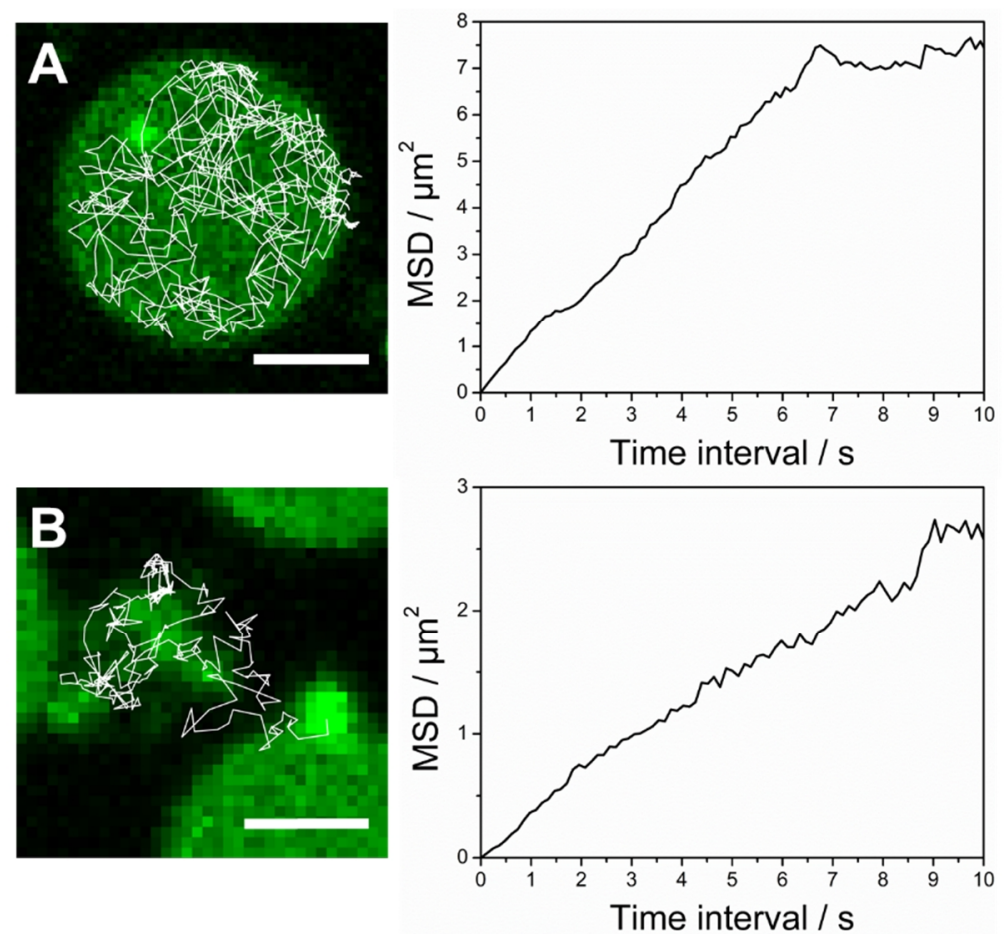
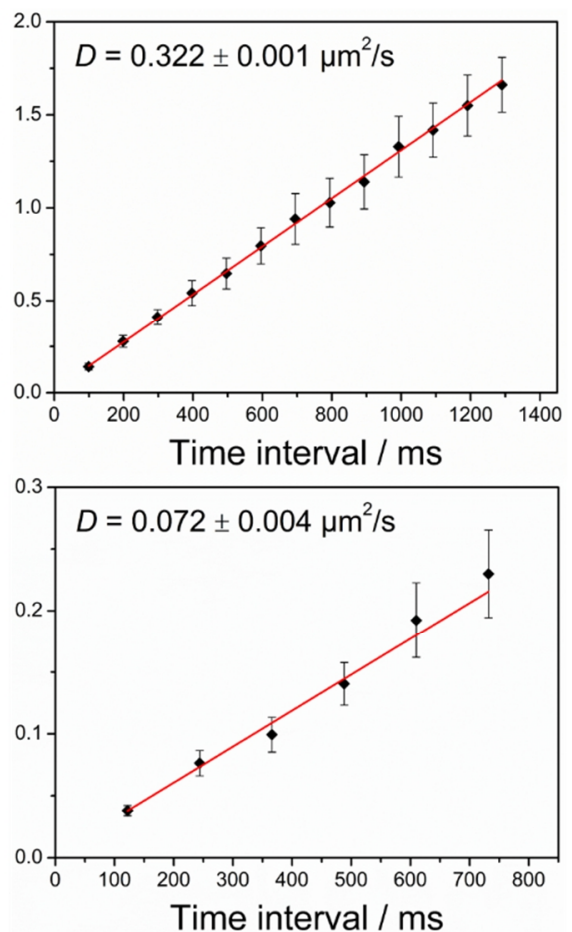

Figure 5-8 Exemplary diffusion trajectories of CG post-fusion structures on both parts of the PSM. (A) Fluorescence micrograph with diffusion trajectory (white line) of a diffusing CG post-fusion on the f-PSM. (scale bar: $2 \mu \mathrm{m}$ ) The MSD vs. $\Delta \mathrm{t}$ plot for time interval of up to $10 \mathrm{~s}$ (middle) with a negative curvature proves the confined diffusion induced by the pore boundary. The diffusion coefficient was obtained from the slope (4D) of a MSD plot against short time intervals (right). (B) Fluorescence micrograph with diffusion trajectory (white line) of a diffusing CG post-fusion on the s-PSM. (scale bar: $2 \mu \mathrm{m}$ ) The MSD vs. $\Delta \mathrm{t}$ plot for time interval of up to $10 \mathrm{~s}$ (middle) shows a linear increase indicating a free diffusion on the s-PSM. The diffusion coefficient was obtained from the slope (4D) of a MSD plot against short time intervals (right).

Concerning the results of the location analysis for CG diffusion post-lipid-mixing (cf. Figure 4-41), the barrier at the pore edge is pronounced in all three mobility states. In the rare cases, in which the CG post-fusion structure managed to leave the pore, the diffusion trajectory seemed to be the result of a scanning process to find the exit (cf. Figure 4-36). The impeded pore exit for CG post-fusion structures might be caused by an enlarged, stalk-like contact area between the CG membrane and the PSM in contrary to only one SNARE-complex or more for a CG in the docking state. However, the pore edge was not observed to be a repelling barrier which would be energy costly to overcome but rather a location with an attractive force. The finding of a higher probability density at the pore edge for CG post-fusion structures, particular in the two low mobility states, supports the hypothesis of an attractive force resulting in energy gain for the CG post-fusion structure at the pore edge. A possible explanation for this observation could be drawn from the topology of the PSM at the pore edge. As monitored by 
atomic force microscopy (AFM) and scanning ion conductance microscopy (SICM), PSMs are not totally flat but exhibit a height difference between the s-PSM and the f-PSM. ${ }^{[171]}$ This height difference of $\sim 100 \mathrm{~nm}$ is most probably the result of the membrane partially following the hydrophilically functionalized solid support, thus bending into the pore (cf. Figure 5-9).

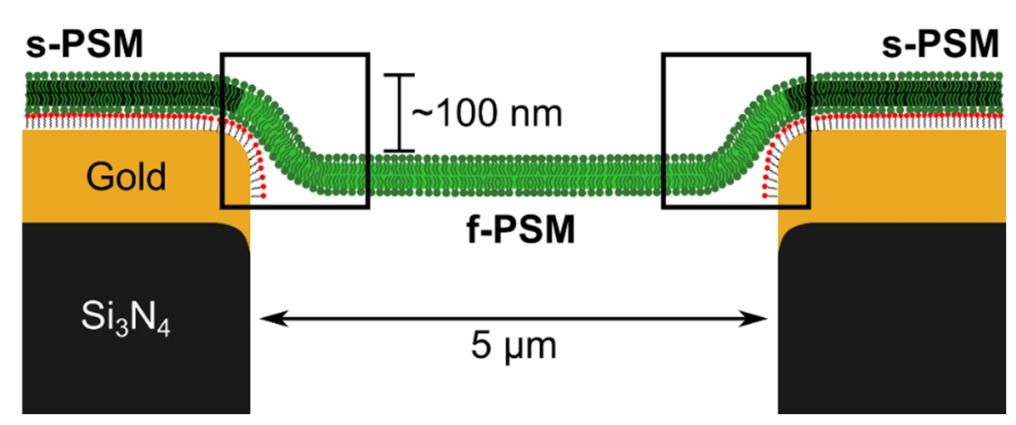

Figure 5-9 Schematic illustration of a cross section of a single pore-spanning membrane. The lipid bilayer partly follows the hydrophilic, 6-MH-functionalized solid support at the edge of the pore before it starts to freely span the pore. The bending of the membrane into the pore results in a height difference of $\sim 100 \mathrm{~nm}$ and leads to a curvature at the edge of the pore highlighted by black boxes.

The curvature at the edge of the f-PSM might lead to a relaxation of the stalk-like contact area of the CG post-fusion structure and further, an adhesive force originating from the solid support could be the reason for an increased probability density at this location. 



\section{Conclusion}

SNARE-mediated membrane fusion is a fundamental cellular process where two initially distinct membranes merge to result in one interconnected structure. In this work, planar porespanning membranes (PSMs) were used to analyze SNARE-mediated membrane fusion on the single particle level by means of confocal laser scanning fluorescence microscopy. The major goal was to investigate the impact of $\mathrm{PI}(4,5) \mathrm{P}_{2}$ and synaptotagmin-1 (syt-1) on SNAREmediated fusion in vitro. The motivation for this study originated from in vivo observations that $\mathrm{PI}(4,5) \mathrm{P}_{2}$ is highly accumulated at sites of fusion and is supposed to sequester the t-SNARE syntaxin-1A (syx-1A) in nanodomains of $\sim 70 \mathrm{~nm}$ in size. ${ }^{[51],[54]}$ Moreover, $\mathrm{PI}(4,5) \mathrm{P}_{2}$ was found to specifically interact with syt- 1 even in the absence of $\mathrm{Ca}^{2+}$, which indicates $\mathrm{PI}(4,5) \mathrm{P}_{2}$ to act as a guide for the $\mathrm{Ca}^{2+}$-sensor to sites of fusion. ${ }^{[16],[103],[148]}$

In a first step, the impact of $\mathrm{PI}(4,5) \mathrm{P}_{2}$ on SNARE-mediated fusion was investigated. For this purpose, the fusion behavior of proteoliposomes with reconstituted synaptobrevin 2 (syb 2) was analyzed on $\triangle \mathrm{N} 49$ doped PSMs varying in their PI(4,5) $\mathrm{P}_{2}$ content of 1,2 and $5 \mathrm{~mol} \%$. For single vesicle fusion on PSMs with these different amounts of $\mathrm{PI}(4,5) \mathrm{P}_{2}$, fusion efficiencies of $\sim 79 \%(1 \mathrm{~mol} \%), \sim 92 \%(2 \mathrm{~mol} \%)$ and $~ 83 \%$ (5 mol\%) were determined, which constitutes a significant increase in comparison to the fusion efficiency of $\sim 50 \%{ }^{[44],[101]}$ monitored on PSMs without $\mathrm{PI}(4,5) \mathrm{P}_{2}$. The average docking lifetime, however, was not found to be considerably affected by the $\mathrm{PI}(4,5) \mathrm{P}_{2}$ content in the PSM. Yet, fitting the obtained docking lifetime distributions with a kinetic model introduced by FLOYD et al. ${ }^{[122]}$, an increased number of hidden steps $N$ for the transition from docking to fusion was determined on PSMs with PI $(4,5) \mathrm{P}_{2}$ $(N=4)$ in comparison to vesicle fusion on PSMs without $\mathrm{PI}(4,5) \mathrm{P}_{2}(N=2)^{[44],[101]}$. These two findings, the increased fusion efficiency and the larger number of hidden steps, are strong indications that SNARE complexes are abundant at sites of vesicle fusion on PSMs, which could be the result of $\mathrm{PI}(4,5) \mathrm{P}_{2}$ induced t-SNARE clustering as observed in vivo.

Since a remarkable fusion efficiency of $\sim 92 \%$ was observed on PSMs containing 2 mol\% $\mathrm{PI}(4,5) \mathrm{P}_{2}$, this content was used for the subsequent analysis of SNARE-mediated single vesicle fusion in the presence of syt-1. Inspired by the observation of JAHN and coworkers ${ }^{[103],[148]}$ that syt-1's mode of interaction with SNAREs and anionic phospholipids is strongly influenced by the ionic strength and the presence of polyphosphates such as ATP, SNARE-mediated single vesicle fusion in the presence of syt-1 was investigated under three different conditions: firstly, as a standard, under $\mathrm{Ca}^{2+}$ - and ATP-free conditions $\left(-\mathrm{Ca}^{2+},-\right.$ ATP), secondly, in the presence 
of $100 \mu \mathrm{M} \mathrm{Ca}^{2+}\left(+\mathrm{Ca}^{2+},-\mathrm{ATP}\right)$ and thirdly, in the presence of both $100 \mu \mathrm{M} \mathrm{Ca}^{2+}$ and $5 \mathrm{mM}$ ATP $\left(+\mathrm{Ca}^{2+},+\mathrm{ATP}\right)$. Under standard conditions $\left(-\mathrm{Ca}^{2+},-\mathrm{ATP}\right)$ syt-1 was found to inhibit SNAREmediated fusion, which was concluded from the determined fusion efficiency of only $\sim 46 \%$. The monitored docking lifetimes, however, were similar to those obtained in the absence of syt-1 under equal conditions. A different behavior was monitored in the presence of $100 \mu \mathrm{M}$ $\mathrm{Ca}^{2+}$. Here, the presence of syt-1 resulted in an increased fusion kinetics as revealed by significantly shorter docking lifetimes (+syt-1: $\tau_{\text {docking }}^{\max }=22 \pm 2 \mathrm{~s},-$ syt $-1: \tau_{\text {docking }}^{\max }=44 \pm 1 \mathrm{~s}$ ). For the fusion efficiency under these conditions $\left(+\mathrm{Ca}^{2+},-\mathrm{ATP}\right)$, however, a slight decrease to $\sim 38 \%$ was determined. In the presence of both $100 \mu \mathrm{M} \mathrm{Ca}^{2+}$ and $5 \mathrm{mM}$ ATP, the syt- 1 induced decrease in fusion efficiency was nearly compensated with a determined percentage of $\sim 82 \%$. The fusion kinetics under these conditions $\left(+\mathrm{Ca}^{2+},+\right.$ ATP $)$ were found to be similar to that observed under $\mathrm{Ca}^{2+}$-only conditions, suggesting a syt-1/Ca ${ }^{2+}$-evoked acceleration of SNAREmediated single vesicle fusion in vitro. Notable is also the observation that the stalled intermediate fusion state was nearly non-existent in the presence of $100 \mu \mathrm{M} \mathrm{Ca}^{2+}$. Under both conditions, $\left(+\mathrm{Ca}^{2+},-\mathrm{ATP}\right)$ and $\left(+\mathrm{Ca}^{2+}+\mathrm{ATP}\right)$, only $\sim 1 \%$ of the fusing vesicles were found to be stalled in the intermediated fusion state, whereas under standard conditions a percentage of $\sim 12 \%$ was determined in the presence of syt- 1 and even $\sim 52 \%$ in its absence.

Aside from analyzing synthetic vesicle fusion, $\Delta \mathrm{N} 49$ doped PSMs with a $\mathrm{PI}(4,5) \mathrm{P}_{2}$ content of $2 \mathrm{~mol} \%$ were also used to investigate the fusion behavior of natural dense core vesicles - the chromaffin granules (CGs). Similar docking lifetimes for CGs on PSMs were observed under standard conditions as for synthetic vesicle fusion, however, by monitoring lipid mixing between CGs and PSMs, a striking difference was found to synthetic vesicles. Whereas the influx of the PSM marker Atto488-DPPE into the vesicle membrane was observed to proceed in a fast, one-step mechanism in case of synthetic vesicle fusion, for CG fusion a two-step mechanism was monitored with a fast first and a slow second process. This difference in synthetic and natural vesicle fusion might originate from a different kind of fusion stalk formation and control of fusion pore dilation. Further, PSMs were observed to be the first planar artificial membrane on which SNARE-bound CGs were not immediately immobilized. For CGs in the docking state, a mean diffusion coefficient of $0.12 \mu \mathrm{m}^{2} / \mathrm{s}$ was determined on the solid supported part (s-PSM), whereas a 3 times higher mobility of $0.34 \mu \mathrm{m}^{2} / \mathrm{s}$ was found on the freestanding part (f-PSM). Interestingly, the observed diverse diffusion behavior of CGs on PSMs is in very good agreement with that, monitored in live chromaffin cells which highlights PSMs to be a valuable model of a plasma membrane. 


\section{$7 \quad$ Appendix}

\subsection{Amino acid sequences of SNAREs and synaptotagmin-1}

\subsubsection{Amino acid sequence of synaptobrevin 2 (syb 2, aa 1-116)}

$\begin{array}{rrrrr}1 \underline{0} & 2 \underline{0} & 3 \underline{0} & 4 \underline{0} & 5 \underline{0} \\ \text { MSATAATVPP } & \text { AAPAGEGGPP } & \text { APPPNLTSNR } & \text { RLQQTQAQVD } & \text { EVVDIMRVNV }\end{array}$

$\begin{array}{rrrrr}\underline{6} \underline{0} & 7 \underline{0} & 8 \underline{0} & 9 \underline{0} & 10 \underline{0} \\ \text { DKVLERDQKL } & \text { SELDDRADAL } & \text { QAGASQFETS } & \text { AAKLKRKYWW } & \text { KNLKMMIILG }\end{array}$

110

VICAIILIII IVYFST

\subsubsection{Amino acid sequence of syntaxin-1A (syx-1A, aa 183-288)}

$\begin{array}{rrrrr}19 \underline{0} & 20 \underline{0} & 21 \underline{0} & 22 \underline{0} & 23 \underline{0} \\ \text { MDSSISKQ } & \text { ALSEIETRHS } & \text { EIIKLENSIR } & \text { ELHDMFMDMA } & \text { MLVESQGEMI } \\ 24 \underline{0} & 25 \underline{0} & 26 \underline{0} & 27 \underline{0} & 28 \underline{0} \\ \text { RIEYNVEHA } & \text { VDYVERAVSD } & \text { TKKAVKYQSK } & \text { ARRKKIMIII } & \text { CCVILGIIIA }\end{array}$

STIGGIFG

\subsubsection{Amino acid sequence of SNAP25a (aa 1-206)}

$\begin{array}{rrrrr}1 \underline{10} & 2 \underline{0} & 3 \underline{0} & 4 \underline{0} & 5 \underline{0} \\ \text { MAEDADMRNE } & \text { LEEMQRRADQ } & \text { LADESLESTR } & \text { RMLQLVEESK } & \text { DAGIRTLVML } \\ \frac{6 \underline{0}}{1} & 7 \underline{0} & 8 \underline{0} & 9 \underline{0} & 10 \underline{0} \\ \text { DEQGEQLERI } & \text { EEGMDQINKD } & \text { MKEAEKNLTD } & \text { LGKFAGLAVA } & \text { PANKLKSSDA } \\ 11 \underline{0} & 12 \underline{0} & 13 \underline{0} & 14 \underline{0} & 15 \underline{0} \\ \text { YKKAWGNNQD } & \text { GVVASQPARV } & \text { VDEREQMAIS } & \text { GGFIRRVTND } & \text { ARENEMDENL } \\ 16 \underline{0} & 17 \underline{0} & 18 \underline{0} & 19 \underline{0} & 20 \underline{0} \\ \text { EQVSGIIGNL } & \text { RHMALDMGNE } & \text { IDTQNRQIDR } & \text { IMEKADSNKT } & \text { RIDEANQRAT }\end{array}$

KMLGSG 


\subsubsection{Amino acid sequence of synaptotagmin-1 (syt-1, aa 1-421)}

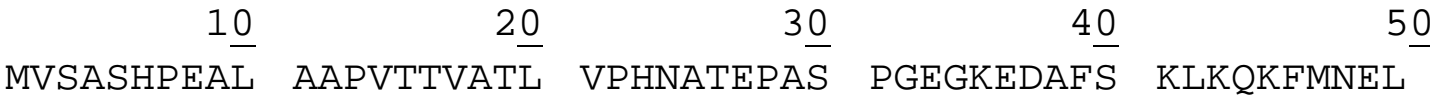

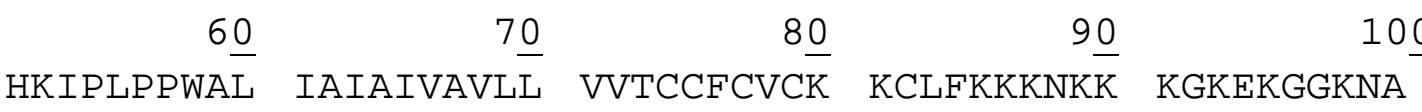

$\begin{array}{rrrrr}11 \underline{0} & 12 \underline{0} & 13 \underline{0} & 14 \underline{0} & 15 \underline{0} \\ \text { INMKDVKDLG } & \text { KTMKDQALKD } & \text { DDAETGLTDG } & \text { EEKEEPKEEE } & \text { KLGKLQYSLD }\end{array}$

$\begin{array}{rrrrr}16 \underline{0} & 17 \underline{0} & 18 \underline{0} & 19 \underline{0} & 20 \underline{0} \\ \text { YDFQNNQLLV } & \text { GIIQAAELPA } & \text { LDMGGTSDPY } & \text { VKVFLLPDKK } & \text { KKFETKVHRK }\end{array}$

$\begin{array}{rrrrr}21 \underline{0} & 22 \underline{0} & 23 \underline{0} & 24 \underline{0} & 25 \underline{0} \\ \text { TLNPVFNEQF } & \text { TFKVPYSELG } & \text { GKTLVMAVYD } & \text { FDRFSKHDI I } & \text { GEFKVPMNTV }\end{array}$

$\begin{array}{lllll}26 \underline{0} & 27 \underline{0} & 28 \underline{0} & 29 \underline{0} & 30 \underline{0}\end{array}$

DFGHVTEEWR DLQSAEKEEQ EKLGDICFSL RYVPTAGKLT VVILEAKNLK

$\begin{array}{lllll}31 \underline{0} & 32 \underline{0} & 33 \underline{0} & 34 \underline{0} & 35 \underline{0}\end{array}$

KMDVGGLSDP YVKIHLMQNG KRLKKKKTTI KKNTLNPYYN ESFSFEVPFE

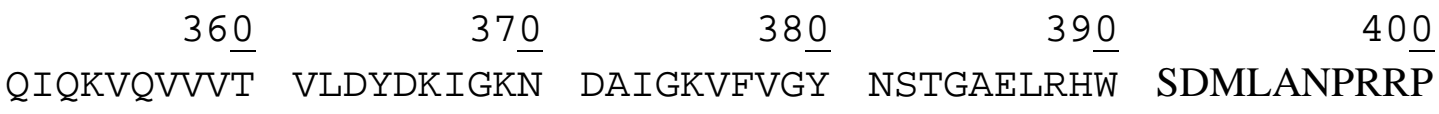

$\begin{array}{rr}410 & 420 \\ \text { IAQWHTLQVE } & \text { EEVDAMLAVK }\end{array}$ 


\subsection{Calculation of the areas of catchment segments Ax for distances $\mathbf{r}_{\mathbf{x}}>\mathbf{r}_{\text {crit }}$}

For CG-pore center distances greater than $r_{\text {crit }}$, the calculation of $A_{\mathrm{x}}$ was more complex as, in this case, the circular segments of the three pores making up a unit cell, start to overlap. A schematic illustration to provide guidance for the following derivation of the catchment area calculations for $r_{\mathrm{x}}>r_{\text {crit }}$ is shown in Figure 7-1.
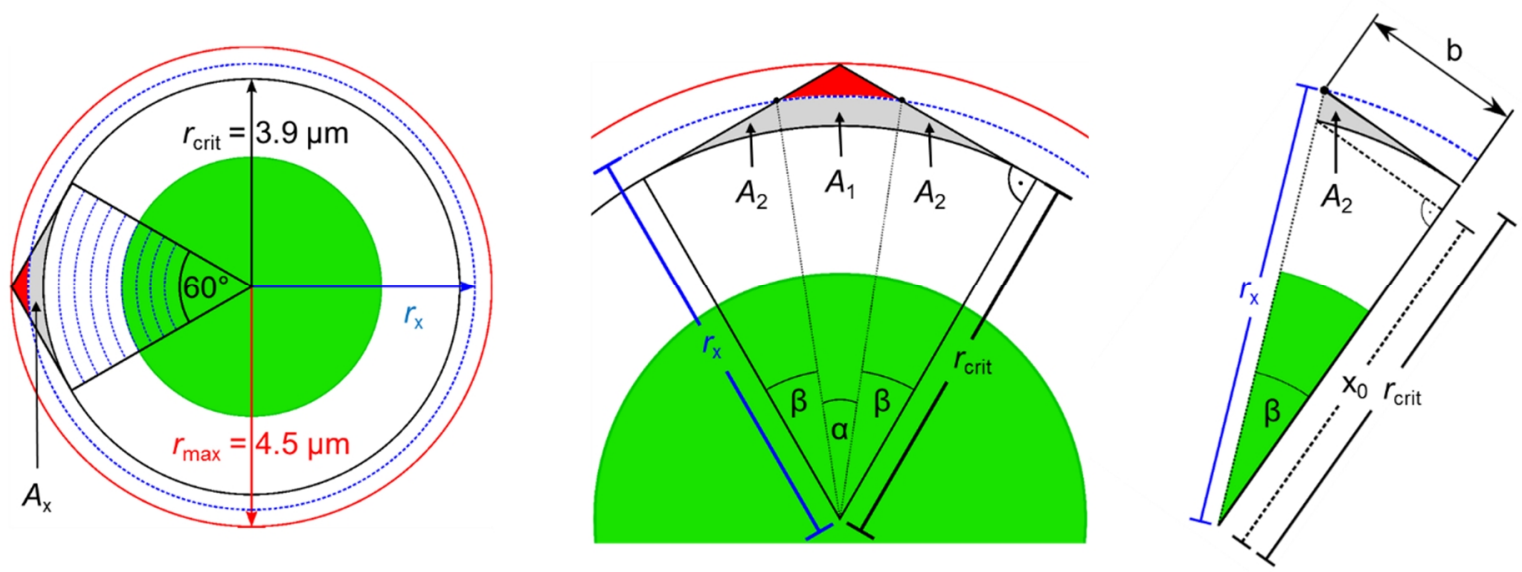

Figure 7-1 Schematic illustrations to provide guidance for the derivation of the calculation of catchment area segments $A_{x}$ for CG-pore center distances $r_{x}>r_{\text {crit. }}$

As the area $A_{1}$ is a type of a circular segment, it was calculated using Equation (7-1).

$$
A_{1}=\pi\left(r_{\mathrm{x}}^{2}-r_{\text {crit }}^{2}\right) \cdot \frac{60^{\circ}-2 \cdot \arccos \left(\frac{r_{\text {crit }}}{r_{\mathrm{x}}}\right)}{360^{\circ}}
$$

For the calculation of $A_{2}$, the following integral was solved

$$
A_{2}=\int_{x_{0}}^{r_{\text {crit }}} \frac{b}{r_{\text {crit }}} \cdot x-\sqrt{r_{\text {crit }}^{2}-x^{2}} d x
$$

with $b=\sqrt{r_{\mathrm{x}}^{2}-r_{\text {crit }}^{2}}$ and $x_{0}=r_{\text {crit }}^{2} / r_{\mathrm{x}}$. For $r_{\mathrm{x}}=r_{\text {crit }}+0.1 \mu \mathrm{m}$, the total area of $A_{\mathrm{x}}$ was calculated as the sum of $A_{1}$ plus two times $A_{2}$. The following segments with an increasing radius in steps of $0.1 \mu \mathrm{m}$ were calculated as shown in Equation (7-3) until the maximum CG-pore center distance of $r_{\text {max }}=r_{\text {crit }} / \cos \left(30^{\circ}\right)=4.5 \mu \mathrm{m}$ was reached.

$$
A_{\mathrm{x}}\left(r_{\mathrm{x}}\right)=A_{1}+2 A_{2}-\left[A_{1}\left(r_{\mathrm{x}}-0.1 \mu \mathrm{m}\right)+2 A_{2}\left(r_{\mathrm{x}}-0.1 \mu \mathrm{m}\right)\right]
$$




\subsection{List of symbols and abbreviations}

\begin{tabular}{|c|c|}
\hline 6-MH & 6-mercaptohexan-1-ol \\
\hline$A$ & area \\
\hline aa & amino acid \\
\hline AFM & atomic force microscope \\
\hline ATP & adenosine triphosphate \\
\hline$c$ & concentration \\
\hline $\mathrm{CG}$ & chromaffin granule \\
\hline CHAPS & 3-[(3-cholamidopropyl)-dimethylammonio]-propanesulfonate \\
\hline Chol & cholesterol \\
\hline CLSM & confocal laser scanning microscopy \\
\hline$D$ & diffusion coefficient \\
\hline$d$ & physical thickness \\
\hline DOPC & 1,2-dioleoyl-sn-glycero-3-phosphocholine \\
\hline DTT & dithiolthreitol \\
\hline E. coli & Escherichia coli \\
\hline FCS & fluorescence correlation spectroscopy \\
\hline f-PSM & freestanding pore spanning membrane \\
\hline FRAP & fluorescence recovery after photobleaching \\
\hline FRET & Förster-Resonance-Energy-Transfer \\
\hline FWHM & full width at half maximum \\
\hline GNP & gold nanoparticle \\
\hline GUV & giant unilamellar vesicle \\
\hline h & hour \\
\hline HEPES & 2-[4-(2-hydroxyethyl)piperazin-1-yl]ethanesulfonic acid \\
\hline His & histidine \\
\hline ITO & indium tin oxide \\
\hline$k$ & rate constant \\
\hline $\mathrm{KCl}$ & sodium chloride \\
\hline $\mathrm{kDa}$ & kilo Dalton \\
\hline LUV & large unilamellar vesicle \\
\hline$M$ & molar mass \\
\hline M & molar \\
\hline $\min$ & minute \\
\hline
\end{tabular}




\begin{tabular}{|c|c|}
\hline ms & milliseconds \\
\hline MSD & mean square displacement \\
\hline MWCO & molecular weight cutoff \\
\hline$n$ & refractive index \\
\hline NSF & N-ethylmaleimide-sensitive factor \\
\hline OT & optical thickness \\
\hline PD & probability density \\
\hline $\mathrm{pI}$ & isoelectric point \\
\hline $\mathrm{PI}(4,5) \mathrm{P}_{2}$ & phosphatidylinositol-4,5-bisphosphate \\
\hline POPE & 1-Palmitoyl-2-oleoyl-sn-glycero-3-phosphoethanolamine \\
\hline POPS & 1-Palmitoyl-2-oleoyl-sn-glycero-3-phospho-1-serine \\
\hline PSM & pore-spanning membrane \\
\hline$r$ & radius \\
\hline$R_{0}$ & FÖRSTER-radius \\
\hline RIfS & reflectrometric interference spectroscopy \\
\hline ROI & region of interest \\
\hline rpm & revolutions per minute \\
\hline RT & room temperature \\
\hline s & second \\
\hline SDCM & spinning disc confocal microscopy \\
\hline SDS-PAGE & sodium dodecyl sulfate polyacrylamide gel electrophoresis \\
\hline SNAP25 & synaptosome associated protein of $25 \mathrm{kDa}$ \\
\hline SNARE & Soluble NSF receptor \\
\hline s-PSM & supported pore-spanning membrane \\
\hline SSM & solid supported membrane \\
\hline SUV & small unilamellar vesicle \\
\hline syb 2 & synaptobrevin 2 \\
\hline syt-1 & synaptotagmin-1 \\
\hline syx-1A & syntaxin-1A \\
\hline$t$ & time \\
\hline TIRF & total internal reflection fluorescence \\
\hline TMD & transmembrane domain \\
\hline TxR-DPPE & TexasRed-DPPE \\
\hline UV & ultraviolett \\
\hline
\end{tabular}




$\begin{array}{ll}\text { vis } & \text { visible } \\ \alpha & \text { incidence angle } \\ \varepsilon & \text { extinction coefficient } \\ \lambda & \text { wave length } \\ \sigma^{2} & \text { variance } \\ \tau_{1 / 2} & \text { half-life } \\ \tau & \text { lifetime }\end{array}$

\subsection{List of chemicals and consumables}

\begin{tabular}{|c|c|}
\hline APS & Sigma-Aldrich (Taufkirchen) \\
\hline Argon & Linde (München) \\
\hline ATP & Sigma-Aldrich (Taufkirchen) \\
\hline Atto488-DPPE & Atto-tec (Siegen) \\
\hline $\mathrm{CaCl}_{2}$ & Merck (Darmstadt) \\
\hline Chloroform & VWR International (Darmstadt) \\
\hline Cholesterol & Sigma-Aldrich (Taufkirchen) \\
\hline Copperband & Präzisions Glas \& Optik GmbH (Iserlohn) \\
\hline DiD-C 18 & $\begin{array}{l}\text { 2-((1E,3E)-5-((Z)-3,3-dimethyl-1-octadecylindolin-2- } \\
\text { ylidene)penta-1,3-dien-1-yl)-3,3-dimethyl-1-octadecyl-3H- } \\
\text { indol-1-ium perchlorate }\end{array}$ \\
\hline DOPC & Avanti Polar Lipids (Alabaster, USA) \\
\hline DTT & Sigma-Aldrich (Taufkirchen) \\
\hline EGTA & Sigma-Aldrich (Taufkirchen) \\
\hline Eppendorf-Cups & Eppendorf (Hamburg) \\
\hline Eppendorfpipettes & Eppendorf (Hamburg) \\
\hline Ethanol & Fluka (Neu-Ulm) \\
\hline Gold, $99.99 \%$ & Allgemeine Gold- und Silberscheideanstalt (Pforzheim) \\
\hline HEPES & Carl Roth GmbH (Karlsruhe) \\
\hline ITO-Slides & Präzisions Glas \& Optik GmbH (Iserlohn) \\
\hline $\mathrm{KCl}$ & Carl Roth GmbH (Karlsruhe) \\
\hline Malachite green oxalate & Alfa Aesar (Karlsruhe) \\
\hline Mucasol@ & Merck (Darmstadt) \\
\hline OG-syx-1A-TMD & Provided by Prof. Dr. Ulf Diederichsen \\
\hline
\end{tabular}




$\begin{array}{ll}\text { Parafilm } & \text { Pechiney Plastik Packaging (Chicago, USA) } \\ \text { Petridishes } & \text { VWR International GmbH, (Nürnberg) } \\ \text { PI(4,5)P } 2 & \text { Echelon Biosciences Inc. (Salt Lake City, USA) } \\ \text { Polycarbonatemembrane } & \text { Avestin (Ontario, Canada) } \\ (400 \mathrm{~nm}+1000 \mathrm{~nm}) & \\ \text { POPE } & \text { Avanti Polar Lipids (Alabaster, USA) } \\ \text { POPS } & \text { Avanti Polar Lipids (Alabaster, USA) } \\ \text { Porous substrates }(1.2 \mu \mathrm{m}) & \text { fluXXion (Eindhofen, The Netherlands) } \\ \text { Porous substrates }(5.0 \mu \mathrm{m}) & \text { Aquamarijn (Zutphen, The Netherlands) } \\ \text { Sucrose } & \text { Carl Roth GmbH (Karlsruhe) } \\ \text { Sulforhodamine B } & \text { Sigma-Adrich (Taufkirchen) } \\ \text { TexasRed-DDPE } & \text { Sigma-Aldrich (Taufkirchen) } \\ \text { Thrombine } & \text { MP Biomedicals (Illkirch, FR) } \\ \text { Titan } & \text { Elektronen Optik Service GmbH (Dortmund) }\end{array}$

\subsection{List of devices and software}

\subsubsection{Devices}

\section{Fluorescence spectroscopy}

JASCO FP 6500

JASCO (Easton, USA)

\section{Fluorescence microscopy}

Olympus BX51

Olympus (Tokio, Japan)

objective:

LumPlan FLN 40x/0.80 W

Olympus (Tokio, Japan)

LSM 710 Examiner

Carl Zeiss GmbH (Jena)

objective:

WPlan-APOChromat 63x/1.0

Carl Zeiss GmbH (Jena)

\section{UV/ vis-spectroscopy}

$\begin{array}{ll}\text { Varian Cary Scan } 50 & \text { Varian (Darmstadt) } \\ \text { Quartz cuvette } & \text { Hellma (Mühlheim) }\end{array}$




\section{Reflectometric interference spectroscopy}

NanoCalc-2000, SD2000

Ocean Optics (Dunedin, USA)

Ismatec $795 \mathrm{C}$

IDEX Health \& Science (Wertheim)

\section{Surface coating}

Zepto plasma cleaner

Diener Electronic, (Ebbhausen)

Coating System MED20

Bal-Tec (Wetzlar)

Sputtercoater 108 auto

Cressington (Watford, UK)

Thickness Controller mtm 20

Cressington (Watfort, UK)

\section{Miscelaneous}

Frequency generator Aglient 333220A

Aglient Technology (Santa Clara, USA)

LiposoFast $^{\circledR}$

Avestin (Ontario, Kanada)

MilliQ Gradient A10

Millipore (Eschborn)

Osmomat 030

Gonotec (Berlin)

Micro scales CP225D

Satorius (Göttingen)

pH meter Calimatic 766

Knick (Berlin)

Galaxy mini

VWR International (Darmstadt)

Concentrator 5301

Eppendorf (Hamburg)

\subsubsection{Software}

Zen 2012

Origin $8.5 \mathrm{G}$

ImageJ $1.45 \mathrm{~s}$

Matlab2014a

Spectra Suite
Carl Zeiss GmbH (Jena)

OriginLab Cooperation (Northahmpton, USA)

http://rsbweb.nih.gov/ij

Math Works (Natick, USA)

Ocean Optics (Dunedin, USA) 


\section{Bibliography}

[1] S.J. Singer \& G.L. Nicolson, The Fluid Mosaic Model of the Structure of Cell Membranes. Science 1972. 175, 720-731.

[2] B. Alberts. Molecular biology of the cell. 5th ed. (Garland Science, New York, 2008).

[3] R. Jahn \& D. Fasshauer, Molecular machines governing exocytosis of synaptic vesicles. Nature 2012. 490, 201-207.

[4] V. Malhotra, L. Orci, B.S. Glick, M.R. Block \& J.E. Rothman, Role of an Nethylmaleimide-sensitive transport component in promoting fusion of transport vesicles with cisternae of the Golgi stack. Cell 1988. 54, 221-227.

[5] M.R. Block, B.S. Glick, C.A. Wilcox, F.T. Wieland \& J.E. Rothman, Purification of an N-ethylmaleimide-sensitive protein catalyzing vesicular transport. Proc Natl Acad Sci USA 1988. 85, 7852-7856.

[6] D.O. Clary, I.C. Griff \& J.E. Rothman, SNAPs, a family of NSF attachment proteins involved in intracellular membrane fusion in animals and yeast. Cell 1990. 61, 709-721.

[7] T. Söllner et al., SNAP receptors implicated in vesicle targeting and fusion. Nature 1993. $362,318-324$.

[8] T. Weimbs et al., A conserved domain is present in different families of vesicular fusion proteins. A new superfamily. Proc Natl Acad Sci USA 1997. 94, 3046-3051.

[9] D. Fasshauer, R.B. Sutton, A.T. Brunger \& R. Jahn, Conserved structural features of the synaptic fusion complex. SNARE proteins reclassified as Q- and R-SNAREs. Proc Natl Acad Sci USA 1998. 95, 15781-15786.

[10] R.B. Sutton, D. Fasshauer, R. Jahn \& A.T. Brunger, Crystal structure of a SNARE complex involved in synaptic exocytosis at 2.4 A resolution. Nature 1998. 395, 347353.

[11] P.I. Hanson, J.E. Heuser \& R. Jahn, Neurotransmitter release — four years of SNARE complexes. Curr Opin Neurobiol 1997. 7, 310-315.

[12] K. Wiederhold \& D. Fasshauer, Is assembly of the SNARE complex enough to fuel membrane fusion? J Biol Chem 2009. 284, 13143-13152.

[13] B.G. Wilhelm et al., Composition of isolated synaptic boutons reveals the amounts of vesicle trafficking proteins. Science 2014. 344, 1023-1028.

[14] J.B. Sørensen, Conflicting views on the membrane fusion machinery and the fusion pore. Annu Rev Cell Dev Biol 2009. 25, 513-537. 
[15] T.C. Südhof, Calcium control of neurotransmitter release. Cold Spring Harb Perspect Biol 2012. 4, a011353.

[16] A. Honigmann et al., Phosphatidylinositol 4,5-bisphosphate clusters act as molecular beacons for vesicle recruitment. Nat Struct Mol Biol 2013. 20, 679-686.

[17] A.T. Brunger, D.J. Cipriano \& J. Diao, Towards reconstitution of membrane fusion mediated by SNAREs and other synaptic proteins. Crit Rev Biochem Mol Biol 2015. 50, 231-241.

[18] D.K. Struck, D. Hoekstra \& R.E. Pagano, Use of resonance energy transfer to monitor membrane fusion. Biochemistry 1981. 20, 4093-4099.

[19] T. Weber et al., SNAREpins. Minimal machinery for membrane fusion. Cell 1998. 92, 759-772.

[20] F. Li, N. Tiwari, J.E. Rothman \& F. Pincet, Kinetic barriers to SNAREpin assembly in the regulation of membrane docking/priming and fusion. Proc Natl Acad Sci USA 2016. 113, 10536-10541.

[21] A.V. Pobbati, A. Stein \& D. Fasshauer, N- to C-terminal SNARE complex assembly promotes rapid membrane fusion. Science 2006. 313, 673-676.

[22] J.M. Hernandez et al., Membrane fusion intermediates via directional and full assembly of the SNARE complex. Science 2012. 336, 1581-1584.

[23] D. Fasshauer \& M. Margittai, A transient N-terminal interaction of SNAP-25 and syntaxin nucleates SNARE assembly. J Biol Chem 2004. 279, 7613-7621.

[24] A. Bhalla, M.C. Chicka, W.C. Tucker \& E.R. Chapman, Ca(2+)-synaptotagmin directly regulates t-SNARE function during reconstituted membrane fusion. Nat Struct Mol Biol 2006. 13, 323-330.

[25] J. Mima, C.M. Hickey, H. Xu, Y. Jun \& W. Wickner, Reconstituted membrane fusion requires regulatory lipids, SNAREs and synergistic SNARE chaperones. EMBO J 2008. 27, 2031-2042.

[26] W. Nickel et al., Content mixing and membrane integrity during membrane fusion driven by pairing of isolated v-SNAREs and t-SNAREs. Proc Natl Acad Sci USA 1999. 96, 12571-12576.

[27] C. Ma, L. Su, A.B. Seven, Y. Xu \& J. Rizo, Reconstitution of the vital functions of Munc18 and Munc13 in neurotransmitter release. Science 2013. 339, 421-425.

[28] J.-Y. Kim et al., Solution single-vesicle assay reveals PIP2-mediated sequential actions of synaptotagmin-1 on SNAREs. EMBO J 2012. 31, 2144-2155. 
[29] B.-K. Choi et al., Large $\alpha$-synuclein oligomers inhibit neuronal SNARE-mediated vesicle docking. Proc Natl Acad Sci USA 2013. 110, 4087-4092.

[30] A. Cypionka et al., Discrimination between docking and fusion of liposomes reconstituted with neuronal SNARE-proteins using FCS. Proc Natl Acad Sci USA 2009. 106, 18575-18580.

[31] M. Kyoung et al., In vitro system capable of differentiating fast Ca2+-triggered content mixing from lipid exchange for mechanistic studies of neurotransmitter release. Proc Natl Acad Sci USA 2011. 108, E304-13.

[32] Y. Lai et al., Complexin inhibits spontaneous release and synchronizes Ca2+-triggered synaptic vesicle fusion by distinct mechanisms. eLife 2014. 3, e03756.

[33] J. Malsam et al., Complexin arrests a pool of docked vesicles for fast Ca2+-dependent release. EMBO J 2012. 31, 3270-3281.

[34] D. Tareste, J. Shen, T.J. Melia \& J.E. Rothman, SNAREpin/Munc18 promotes adhesion and fusion of large vesicles to giant membranes. Proc Natl Acad Sci USA 2008. 105, 2380-2385.

[35] A. Witkowska \& R. Jahn, Rapid SNARE-Mediated Fusion of Liposomes and Chromaffin Granules with Giant Unilamellar Vesicles. Biophys J 2017. 113, 12511259.

[36] M.E. Bowen, K. Weninger, A.T. Brunger \& S. Chu, Single molecule observation of liposome-bilayer fusion thermally induced by soluble N-ethyl maleimide sensitivefactor attachment protein receptors (SNAREs). Biophys J 2004. 87, 3569-3584.

[37] M. Fix et al., Imaging single membrane fusion events mediated by SNARE proteins. Proc Natl Acad Sci USA 2004. 101, 7311-7316.

[38] T. Liu, W.C. Tucker, A. Bhalla, E.R. Chapman \& J.C. Weisshaar, SNARE-driven, 25millisecond vesicle fusion in vitro. Biophys $J$ 2005. 89, 2458-2472.

[39] A.J.B. Kreutzberger, V. Kiessling \& L.K. Tamm, High cholesterol obviates a prolonged hemifusion intermediate in fast SNARE-mediated membrane fusion. Biophys $J \mathbf{2 0 1 5}$. 109, 319-329.

[40] R.J. Rawle, B. van Lengerich, M. Chung, P.M. Bendix \& S.G. Boxer, Vesicle fusion observed by content transfer across a tethered lipid bilayer. Biophys $J$ 2011. 101, L379.

[41] E. Karatekin \& J.E. Rothman, Fusion of single proteoliposomes with planar, cushioned bilayers in microfluidic flow cells. Nat Protoc 2012. 7, 903-920. 
[42] M.K. Domanska, V. Kiessling, A. Stein, D. Fasshauer \& L.K. Tamm, Single vesicle millisecond fusion kinetics reveals number of SNARE complexes optimal for fast SNARE-mediated membrane fusion. J Biol Chem 2009. 284, 32158-32166.

[43] I. Höfer \& C. Steinem, A membrane fusion assay based on pore-spanning lipid bilayers. Soft Matter 2011. 7, 1644.

[44] L.L.G. Schwenen et al., Resolving single membrane fusion events on planar porespanning membranes. Sci Rep 2015. 5, 12006.

[45] J.W. Kuhlmann, M. Junius, U. Diederichsen \& C. Steinem, SNARE-Mediated SingleVesicle Fusion Events with Supported and Freestanding Lipid Membranes. Biophys $J$ 2017. 112, 2348-2356.

[46] hebbery.wordpress.com, Electron micrograph of a chemical synapse. Available at https://hebbery.files.wordpress.com/2014/11/c983c-synapse.jpg, [Online: 28.11.2017].

[47] S. McLaughlin, J. Wang, A. Gambhir \& D. Murray, PIP(2) and proteins. Interactions, organization, and information flow. Апnи Rev Biophys Biomol Struct 2002. 31, 151175.

[48] M.J. Berridge \& R.F. Irvine, Inositol trisphosphate, a novel second messenger in cellular signal transduction. Nature 1984. 312, 315-321.

[49] G. Kabachinski, M. Yamaga, D.M. Kielar-Grevstad, S. Bruinsma \& T.F.J. Martin, CAPS and Munc13 utilize distinct PIP2-linked mechanisms to promote vesicle exocytosis. Mol Biol Cell 2014. 25, 508-521.

[50] D.J. James, C. Khodthong, J.A. Kowalchyk \& T.F.J. Martin, Phosphatidylinositol 4,5bisphosphate regulates SNARE-dependent membrane fusion. J Cell Biol 2008. 182, 355-366.

[51] G. van den Bogaart et al., Membrane protein sequestering by ionic protein-lipid interactions. Nature 2011. 479, 552-555.

[52] D. Milovanovic et al., Hydrophobic mismatch sorts SNARE proteins into distinct membrane domains. Nat Commun 2015. 6, 5984.

[53] D.H. Murray \& L.K. Tamm, Clustering of syntaxin-1A in model membranes is modulated by phosphatidylinositol 4,5-bisphosphate and cholesterol. Biochemistry 2009. 48, 4617-4625.

[54] D. Milovanovic et al., Calcium Promotes the Formation of Syntaxin 1 Mesoscale Domains through Phosphatidylinositol 4,5-Bisphosphate. J Biol Chem 2016. 291, 78687876. 
[55] J.E. Heuser \& T.S. Reese, Evidence for recycling of synaptic vesicle membrane during transmitter release at the frog neuromuscular junction. J Cell Biol 1973. 57, 315-344.

[56] B. Katz \& R. Miledi, The timing of calcium action during neuromuscular transmission. J Physiol 1967. 189, 535-544.

[57] R.W. Tsien, P.T. Ellinor \& W.A. Horne, Molecular diversity of voltage-dependent Ca2+ channels. Trends Pharmacol Sci 1991. 12, 349-354.

[58] S. Hefft \& P. Jonas, Asynchronous GABA release generates long-lasting inhibition at a hippocampal interneuron-principal neuron synapse. Nat Neurosci 2005. 8, 1319-1328.

[59] D.E. Knight, H. von Grafenstein \& C.M. Athayde, Calcium-dependent and calciumindependent exocytosis. Trends Neurosci 1989. 12, 451-458.

[60] J. Sun et al., A dual-Ca2+-sensor model for neurotransmitter release in a central synapse. Nature 2007. 450, 676-682.

[61] M. Geppert et al., Synaptotagmin I. A major Ca2+ sensor for transmitter release at a central synapse. Cell 1994. 79, 717-727.

[62] R. Fernández-Chacón et al., Synaptotagmin I functions as a calcium regulator of release probability. Nature 2001. 410, 41-49.

[63] M.C. Chicka, E. Hui, H. Liu \& E.R. Chapman, Synaptotagmin arrests the SNARE complex before triggering fast, efficient membrane fusion in response to $\mathrm{Ca} 2+$. Nat Struct Mol Biol 2008. 15, 827-835.

[64] T.C. Rao et al., Synaptotagmin isoforms confer distinct activation kinetics and dynamics to chromaffin cell granules. J Gen Physiol 2017. 149, 763-780.

[65] W.D. Matthew, L. Tsavaler \& L.F. Reichardt, Identification of a synaptic vesiclespecific membrane protein with a wide distribution in neuronal and neurosecretory tissue. J Cell Biol 1981. 91, 257-269.

[66] A. Radhakrishnan, A. Stein, R. Jahn \& D. Fasshauer, The Ca2+ affinity of synaptotagmin 1 is markedly increased by a specific interaction of its $\mathrm{C} 2 \mathrm{~B}$ domain with phosphatidylinositol-4,5-bisphosphate. J Biol Chem 2009. 284, 25749-25760.

[67] X. Shao, I. Fernandez, T.C. Südhof \& J. Rizo, Solution structures of the Ca2+-free and $\mathrm{Ca} 2+-$ bound C2A domain of synaptotagmin I. Does Ca2+ induce a conformational change? Biochemistry 1998. 37, 16106-16115.

[68] Y. Cheng et al., Crystallographic identification of $\mathrm{Ca} 2+$ and $\mathrm{Sr} 2+$ coordination sites in synaptotagmin I C2B domain. Protein Sci 2004. 13, 2665-2672.

[69] E.R. Chapman, Synaptotagmin. A Ca(2+) sensor that triggers exocytosis? Nat Rev Mol Cell Biol 2002. 3, 498-508. 
[70] N. Brose, A.G. Petrenko, T.C. Südhof \& R. Jahn, Synaptotagmin. A calcium sensor on the synaptic vesicle surface. Science 1992. 256, 1021-1025.

[71] X. Shao et al., Synaptotagmin-Syntaxin Interaction. The C2 Domain as a Ca2+Dependent Electrostatic Switch. Neuron 1997. 18, 133-142.

[72] S. Martens \& H.T. McMahon, Mechanisms of membrane fusion. Disparate players and common principles. Nat Rev Mol Cell Biol 2008. 9, 543-556.

[73] W.B. Cannon. Bodily changes in pain, hunger, fear and rage. An account of recent researches into the function of emotional excitement (D. Appleton \& Co., New York and London, 1915).

[74] W.B. Cannon. The wisdom of the body (Norton, New York, 1967).

[75] A.S.P. Jansen, X.V. Nguyen, V. Karpitskiy, T.C. Mettenleiter \& A.D. Loewy, Central Command Neurons of the Sympathetic Nervous System. Basis of the Fight-or-Flight Response. Science 1995. 270, 644-646.

[76] T. Fulop, S. Radabaugh \& C. Smith, Activity-dependent differential transmitter release in mouse adrenal chromaffin cells. J Neurosci 2005. 25, 7324-7332.

[77] H.S. Bennett, Cytological manifestations of secretion in the adrenal medulla of the cat. Am. J. Anat. 1941. 69, 333-381.

[78] E. Crivellato, B. Nico \& D. Ribatti, The chromaffin vesicle: advances in understanding the composition of a versatile, multifunctional secretory organelle. Anat Rec (Hoboken) 2008. 291, 1587-1602.

[79] A. Hodel, T. Schäfer, D. Gerosa \& M.M. Burger, In chromaffin cells, the mammalian Sec1p homologue is a syntaxin 1A-binding protein associated with chromaffin granules. J Biol Chem 1994. 269, 8623-8626.

[80] I. López et al., Vesicle motion and fusion are altered in chromaffin cells with increased SNARE cluster dynamics. Traffic 2009. 10, 172-185.

[81] I. López-Font, C.J. Torregrosa-Hetland, J. Villanueva \& L.M. Gutiérrez, t-SNARE cluster organization and dynamics in chromaffin cells. J Neurochem 2010. 114, 15501556.

[82] M. Ceridono et al., Selective recapture of secretory granule components after full collapse exocytosis in neuroendocrine chromaffin cells. Traffic 2011. 12, 72-88.

[83] S.-A. Chan, B. Doreian \& C. Smith, Dynamin and myosin regulate differential exocytosis from mouse adrenal chromaffin cells. Cell Mol Neurobiol 2010. 30, 13511357. 
[84] A. Llobet, M. Wu \& L. Lagnado, The mouth of a dense-core vesicle opens and closes in a concerted action regulated by calcium and amphiphysin. J Cell Biol 2008. 182, $1017-1028$.

[85] D. Perrais, I.C. Kleppe, J.W. Taraska \& W. Almers, Recapture after exocytosis causes differential retention of protein in granules of bovine chromaffin cells. J Physiol 2004. $560,413-428$.

[86] S. Houy et al., Exocytosis and endocytosis in neuroendocrine cells: inseparable membranes! Front Endocrinol 2013. 4, 135.

[87] K. Diba, A. Amarasingham, K. Mizuseki \& G. Buzsáki, Millisecond timescale synchrony among hippocampal neurons. J Neurosci 2014. 34, 14984-14994.

[88] G.J. Augustine, F. Santamaria \& K. Tanaka, Local Calcium Signaling in Neurons. Neuron 2003. 40, 331-346.

[89] J. Lee \& J.T. Littleton, Transmembrane tethering of synaptotagmin to synaptic vesicles controls multiple modes of neurotransmitter release. Proc Natl Acad Sci USA 2015. 112, 3793-3798.

[90] J.W. Kuhlmann, Dissertation, Modulation of lateral membrane tension and SNAREmediated single vesicle fusion on pore-spanning membranes, Georg-August-Universität 2017.

[91] W.C. Breckenridge, I.G. Morgan, J.P. Zanetta \& G. Vincendon, Adult rat brain synaptic vesicles II. Lipid composition. Biochim Biophys Acta 1973. 320, 681-686.

[92] C.W. Cotman, M.L. Blank, A. Moehl \& F. Snyder, Lipid composition of synaptic plasma membranes isolated from rat brain by zonal centrifugation. Biochemistry 2002. 8, 4606-4612.

[93] UniProt, secondary structure SNAP25a (1-206). Available at http://www.uniprot.org/uniprot/P60881, [Online: 28.11.2017].

[94] I. Dulubova et al., A conformational switch in syntaxin during exocytosis: role of munc18. EMBO J 1999. 18, 4372-4382.

[95] M. Margittai et al., Single-molecule fluorescence resonance energy transfer reveals a dynamic equilibrium between closed and open conformations of syntaxin 1. Proc Natl Acad Sci USA 2003. 100, 15516-15521.

[96] UniProt, secondary structure syntaxin-1A (183-288). Available at http://www.uniprot.org/uniprot/P32851, [Online: 28.11.2017].

[97] UniProt, secondary structure synaptobrevin 2 (1-116). Available at http://www.uniprot.org/uniprot/P63045, [Online: 28.11.2017]. 
[98] UniProt, secondary structure of syt-1 (1-421). Available at http://www.uniprot.org/uniprot/P21707, [Online: 28.11.2017].

[99] H. Schägger \& G. von Jagow, Tricine-sodium dodecyl sulfate-polyacrylamide gel electrophoresis for the separation of proteins in the range from 1 to $100 \mathrm{kDa}$. Anal Biochem 1987. 166, 368-379.

[100] H. Schägger, Tricine-SDS-PAGE. Nat Protoc 2006. 1, 16-22.

[101] L.L.G. Schwenen, Dissertation, Untersuchung einzelner SNARE-vermittelter Membranfusionsereignisse auf planaren porenüberspannenden Membranen, GeorgAugust-Universität 2015.

[102] J.M. Hernandez, Dissertation, Reconstituted SNARE-mediated fusion: towards a mechanistic understanding, Georg-August-Universität 2011.

[103] Y. Park et al., Controlling synaptotagmin activity by electrostatic screening. Nat Struct Mol Biol 2012. 19, 991-997.

[104] T. Attin, K. Becker, C. Hannig, W. Buchalla \& A. Wiegand, Suitability of a malachite green procedure to detect minimal amounts of phosphate dissolved in acidic solutions. Clin Oral Investig 2005. 9, 203-207.

[105] J. Feng et al., An improved malachite green assay of phosphate: mechanism and application. Anal Biochem 2011. 409, 144-149.

[106] G. Wedler \& H.-J. Freund eds. Lehrbuch der physikalischen Chemie (Wiley-VCH, Weinheim, 2012).

[107] M. Nič, J. Jirát, B. Košata, A. Jenkins \& A. McNaught. IUPAC Compendium of Chemical Terminology (IUPAC, Research Triagle Park, NC, 2009).

[108] M. Schwamborn, Dissertation, Establishment of a fluorescence assay for characterization of protein-mediated vesicle fusion and acidification, Georg-AugustUniversität 2017.

[109] J.R. Lakowicz. Principles of Fluorescence Spectroscopy (Springer US, Boston, MA, 2006).

[110] J.W. Lichtman \& J.-A. Conchello, Fluorescence microscopy. Nat Methods 2005. 2, 910-919.

[111] O.M. Schütte, Dissertation, Structure and dynamics of artificial lipid membranes containing the glycosphingolipid Gb3, Georg-August-Universität 2015.

[112] Wilhelm, S., Gröbler, B., Gluch, M. \& Heinz, H., Confocal Laser Scanning Microscopy. Principles. 
campus.magnet.fsu.edu/referencelibrary/pdfs/ZeissConfocalPrinciples.pdf, [Online: 28.11.2017].

[113] S.C. Stein \& J. Thiart, TrackNTrace: A simple and extendable open-source framework for developing single-molecule localization and tracking algorithms. Sci Rep 2016. 6, 37947.

[114] G. Gauglitz, Direct optical sensors: principles and selected applications. Anal Bioanal Chem 2005. 381, 141-155.

[115] F. Baldini. Optical chemical sensors (Springer, Dordrecht, 2006).

[116] O.S. Heavens, Optical properties of thin films. Rep Prog Phys 1960. 23, 1-65.

[117] J. Vörös, The density and refractive index of adsorbing protein layers. Biophys $J \mathbf{2 0 0 4}$. 87, 553-561.

[118] K. Tawa \& K. Morigaki, Substrate-supported phospholipid membranes studied by surface plasmon resonance and surface plasmon fluorescence spectroscopy. Biophys $J$ 2005. 89, 2750-2758.

[119] R. Krick et al., Structural and functional characterization of the two phosphoinositide binding sites of PROPPINs, a $\beta$-propeller protein family. Proc Natl Acad Sci USA 2012. 109, E2042-9.

[120] F. Benfenati, Electrostatic and hydrophobic interactions of synapsin I and synapsin I fragments with phospholipid bilayers. J Cell Biol 1989. 108, 1851-1862.

[121] D. Freedman \& P. Diaconis, On the histogram as a density estimator: L2 theory. Z. Wahrscheinlichkeitstheorie verw Gebiete 1981. 57, 453-476.

[122] D.L. Floyd, S.C. Harrison \& A.M. van Oijen, Analysis of kinetic intermediates in singleparticle dwell-time distributions. Biophys $J$ 2010. 99, 360-366.

[123] J.A. Braunger, C. Kramer, D. Morick \& C. Steinem, Solid supported membranes doped with PIP2 - influence of ionic strength and $\mathrm{pH}$ on bilayer formation and membrane organization. Langmuir 2013. 29, 14204-14213.

[124] P.F. Baker \& D.E. Knight, Calcium-dependent exocytosis in bovine adrenal medullary cells with leaky plasma membranes. Nature 1978. 276, 620-622.

[125] A.T. Brunger, K. Weninger, M. Bowen \& S. Chu, Single-molecule studies of the neuronal SNARE fusion machinery. Annual review of biochemistry 2009. 78, 903-928.

[126] M. Tagaya, T. Genma, A. Yamamoto, S. Kozaki \& S. Mizushima, SNAP-25 is present on chromaffin granules and acts as a SNAP receptor. FEBS Letters 1996. 394, 83-86. 
[127] G. Schneider et al., Distance-dependent fluorescence quenching on gold nanoparticles ensheathed with layer-by-layer assembled polyelectrolytes. Nano letters 2006. 6, 530536.

[128] C. Chen et al., Size and distance dependent fluorescence enhancement of nanoporous gold. Optics express 2017. 25, 9901-9910.

[129] J.A. Steyer \& W. Almers, Tracking Single Secretory Granules in Live Chromaffin Cells by Evanescent-Field Fluorescence Microscopy. Biophys J 1999. 76, 2262-2271.

[130] M. Oheim \& W. Stühmer, Tracking chromaffin granules on their way through the actin cortex. European biophysics journal : EBJ 2000. 29, 67-89.

[131] S. Huet et al., Analysis of transient behavior in complex trajectories. Application to secretory vesicle dynamics. Biophys J 2006. 91, 3542-3559.

[132] M.W. Allersma, M.A. Bittner, D. Axelrod \& R.W. Holz, Motion matters. Secretory granule motion adjacent to the plasma membrane and exocytosis. Mol Biol Cell 2006. 17, 2424-2438.

[133] T.F.J. Martin, PI(4,5)P2 regulation of surface membrane traffic. Current Opinion in Cell Biology 2001. 13, 493-499.

[134] V. Shabardina et al., Mode of Ezrin-Membrane Interaction as a Function of PIP2 Binding and Pseudophosphorylation. Biophys J 2016. 110, 2710-2719.

[135] J.A. Braunger et al., Phosphatidylinositol 4,5-bisphosphate alters the number of attachment sites between ezrin and actin filaments. A colloidal probe study. J Biol Chem 2014. 289, 9833-9843.

[136] G. van den Bogaart \& R. Jahn, Counting the SNAREs needed for membrane fusion. Journal of molecular cell biology 2011. 3, 204-205.

[137] M. Toner, G. Vaio, A. McLaughlin \& S. McLaughlin, Adsorption of cations to phosphatidylinositol 4,5-bisphosphate. Biochemistry 1988. 27, 7435-7443.

[138] D. Lupyan, M. Mezei, D.E. Logothetis \& R. Osman, A molecular dynamics investigation of lipid bilayer perturbation by PIP2. Biophys J 2010. 98, 240-247.

[139] E. Karatekin et al., A fast, single-vesicle fusion assay mimics physiological SNARE requirements. Proc Natl Acad Sci USA 2010. 107, 3517-3521.

[140] Y. Hua \& R.H. Scheller, Three SNARE complexes cooperate to mediate membrane fusion. Proc Natl Acad Sci USA 2001. 98, 8065-8070.

[141] X. Han, C.-T. Wang, J. Bai, E.R. Chapman \& M.B. Jackson, Transmembrane segments of syntaxin line the fusion pore of Ca2+-triggered exocytosis. Science 2004. 304, 289292. 
[142] R. Mohrmann, H. de Wit, M. Verhage, E. Neher \& J.B. Sørensen, Fast vesicle fusion in living cells requires at least three SNARE complexes. Science 2010. 330, 502-505.

[143] A. Stein, Dissertation, Untersuchung zur Rekonstitution der SNARE-vermittelten Membranfusion, Freie Universität Berlin 2008.

[144] A. Cypionka, Dissertation, Neue Einblicke in die SNARE-vermittelte Fusion: Detektion einzelner Proteoliposomen mit einem konfokalen Mikroskop, Georg-AugustUniversität 2009.

[145] A.J.B. Kreutzberger, B. Liang, V. Kiessling \& L.K. Tamm, Assembly and Comparison of Plasma Membrane SNARE Acceptor Complexes. Biophys J 2016. 110, 2147-2150.

[146] J. Bai, W.C. Tucker \& E.R. Chapman, PIP2 increases the speed of response of synaptotagmin and steers its membrane-penetration activity toward the plasma membrane. Nat Struct Mol Biol 2004. 11, 36-44.

[147] Á. Pérez-Lara et al., PtdInsP2 and PtdSer cooperate to trap synaptotagmin-1 to the plasma membrane in the presence of calcium. eLife 2016. 5.

[148] Y. Park et al., Synaptotagmin-1 binds to PIP(2)-containing membrane but not to SNAREs at physiological ionic strength. Nat Struct Mol Biol 2015. 22, 815-823.

[149] C.-C. Lin et al., Control of membrane gaps by synaptotagmin-Ca2+ measured with a novel membrane distance ruler. Nat Commun 2014. 5, 5859.

[150] W. Vennekate et al., Cis- and trans-membrane interactions of synaptotagmin-1. Proc Natl Acad Sci USA 2012. 109, 11037-11042.

[151] K.C. Martin et al., Evidence for synaptotagmin as an inhibitory clamp on synaptic vesicle release in Aplysia neurons. Proc Natl Acad Sci USA 1995. 92, 11307-11311.

[152] G. Schiavo, G. Stenbeck, J.E. Rothman \& T.H. Söllner, Binding of the synaptic vesicle v-SNARE, synaptotagmin, to the plasma membrane t-SNARE, SNAP-25, can explain docked vesicles at neurotoxin-treated synapses. Proc Natl Acad Sci USA 1997. 94, 9971001 .

[153] C. Rickman \& B. Davletov, Mechanism of calcium-independent synaptotagmin binding to target SNAREs. J Biol Chem 2003. 278, 5501-5504.

[154] C. Rickman et al., Conserved prefusion protein assembly in regulated exocytosis. Mol Biol Cell 2006. 17, 283-294.

[155] H. de Wit et al., Synaptotagmin-1 docks secretory vesicles to syntaxin-1/SNAP-25 acceptor complexes. Cell 2009. 138, 935-946.

[156] Q. Zhou et al., Architecture of the synaptotagmin-SNARE machinery for neuronal exocytosis. Nature 2015. 525, 62-67. 
[157] K.D. Brewer et al., Dynamic binding mode of a Synaptotagmin-1-SNARE complex in solution. Nat Struct Mol Biol 2015. 22, 555-564.

[158] A.W. Henkel, G. Kang \& J. Kornhuber, A common molecular machinery for exocytosis and the 'kiss-and-run' mechanism in chromaffin cells is controlled by phosphorylation. J Cell Sci 2001. 114, 4613-4620.

[159] S.T. Hanna et al., Kiss-and-run exocytosis and fusion pores of secretory vesicles in human beta-cells. Pflugers Arch 2009. 457, 1343-1350.

[160] H. Plattner, A.R. Artalejo \& E. Neher, Ultrastructural organization of bovine chromaffin cell cortex-analysis by cryofixation and morphometry of aspects pertinent to exocytosis. J Cell Biol 1997. 139, 1709-1717.

[161] A.J.B. Kreutzberger et al., Asymmetric Phosphatidylethanolamine Distribution Controls Fusion Pore Lifetime and Probability. Biophys J 2017. 113, 1912-1915.

[162] A.J.B. Kreutzberger et al., Reconstitution of calcium-mediated exocytosis of dense-core vesicles. Science advances 2017. 3, e1603208.

[163] H.-C. Chiang et al., Post-fusion structural changes and their roles in exocytosis and endocytosis of dense-core vesicles. Nat Commun 2014. 5, 77.

[164] W.-D. Zhao et al., Hemi-fused structure mediates and controls fusion and fission in live cells. Nature 2016. 534, 548-552.

[165] E. Alés et al., High calcium concentrations shift the mode of exocytosis to the kiss-andrun mechanism. Nature cell biology 1999. 1, 40-44.

[166] A. Albillos et al., The exocytotic event in chromaffin cells revealed by patch amperometry. Nature 1997. 389, 509-512.

[167] J.M. Cabeza, J. Acosta \& E. Alés, Mechanisms of granule membrane recapture following exocytosis in intact mast cells. J Biol Chem 2013. 288, 20293-20305.

[168] S. Spindler, Dissertation, Ultrasensitive detection and tracking of nanoparticles on model lipid membrane systems by interferometric scattering microscopy, FriedrichAlexander Universität Erlangen-Nürnberg 2017.

[169] S. Nofal, U. Becherer, D. Hof, U. Matti \& J. Rettig, Primed vesicles can be distinguished from docked vesicles by analyzing their mobility. J Neurosci 2007. 27, 1386-1395.

[170] K. Berberian, A.J. Torres, Q. Fang, K. Kisler \& M. Lindau, F-actin and myosin II accelerate catecholamine release from chromaffin granules. J Neurosci 2009. 29, 863870. 
[171] M. Böcker, S. Muschter, E.K. Schmitt, C. Steinem \& T.E. Schäffer, Imaging and patterning of pore-suspending membranes with scanning ion conductance microscopy. Langmuir : the ACS journal of surfaces and colloids 2009. 25, 3022-3028. 



\section{Curriculum vitae}

\section{Personal data}

$\begin{array}{ll}\text { Name: } & \text { Raphael Hubrich } \\ \text { Date of birth: } & 23.02 .1989 \\ \text { Place of birth: } & \text { Bremen, Germany } \\ \text { Citizenship: } & \text { German }\end{array}$

\section{Carreer}

since 12/2013 Ph.D. thesis at the Georg-August University in Göttingen, entitled "Pore-spanning membranes - a versatile tool to analyze SNAREmediated single vesicle fusion”, under leadership of PROF. DR. C. STEINEM.

10/2011-10/2013 Graduate studies in chemistry at the Georg-August University in Göttingen, degree: Master of Science, grade: 1.5, title of the master thesis: "Reconstitution of SNARE-proteins into pore-spanning membranes and determination of their lateral mobility”, grade: 1.0

10/2008-09/2011 Undergraduate studies in chemistry at the Georg-August University in Göttingen, degree: Bachelor of Science, grade: 1.9, title of the bachelor thesis: "Quantifying the interaction between c-Polycystin-2-S812D and PIGEA-14 by means of quartz crystal microbalance”, grade: 1.0

08/2001-07/2008 Allgemeine Hochschulreife at Lessing Gymnasium in Uelzen, degree: Abitur (advanced courses: Chemistry, Biology, English), grade: 2.4

\section{Scientific contributions}

\section{Peer-reviewed publications}

07/2015 L.L.G. Schwenen, R. Hubrich, D. Milovanovic, B. Geil, J. Yang, A. Kros, R. Jahn, C. Steinem, Sci. Rep. 2015, 5 (12006).

07/2013 D. Behn, M. Schatz, R. Hubrich, H. Hoffmeister, A. Kreff, R. Witzgall, C. Steinem, Biochim. Biophys. Res. Comm. 2013, 437, 532-537. 


\section{Oral presentation}

10/2015 SFB 803 Autumn School in Altenau, Germany. Title of the presentation: "Synapse on a Chip: SNARE-mediated fusion on planar pore-spanning membranes"

\section{Selected poster presentations}

02/2016 Biophysical Society $60^{\text {th }}$ Annual Meeting in Los Angeles, USA. Title: "Synapse on a Chip: SNARE-mediated fusion on planar pore-spanning membranes"

07/2015 10 $10^{\text {th }}$ European Biophysics Congress in Dresden, Germany. Title: "Synapse on a Chip: SNARE-mediated fusion on planar pore-spanning membranes"

* Winner of the Student Poster Award

04/2015 585. WE-Heraeus-Seminar in Bad Honnef, Germany. Title: "Synapse on a Chip: SNARE-mediated fusion on planar pore-spanning membranes" 


\section{Danksagung}

An erster Stelle möchte ich mich ausdrücklich bei PROF. DR. ClaUdia STEINEM für die gute Betreuung während dieser Arbeit und für die zahlreichen fachlichen Diskussionen bedanken.

Ich danke den Mitgliedern meines Thesis Committees, Prof. Dr. ReInHARd JAHN und Prof. DR. ULF DIEDERICHSEN für fruchtbare Ideen und den guten wissenschaftlichen Austausch.

Ein besonderer Dank geht auch an INGO, ohne dessen Hilfe Matlab mich in den Wahnsinn getrieben hätte! JERRY und JOHANNES seien an dieser Stelle auch erwähnt - vielen Dank!

Für die Unterstützung bei diversen Laborarbeiten möchte ich mich bei JUTTA und MicHA bedanken. Unseren Sekretärinnen DANA, MELANIE und MARIANNE danke ich für die stetige Unterstützung und Engelsgeduld beim Einreichen sämtlicher Formulare sowie für die Hilfe bei vielen anderen organisatorischen Dingen.

Weiterhin möchte ich mich bei meinem Masterstudenten STEFAN und meinen zwei Bachelorstudentinnen FRANZI und KITA bedanken für die Motivation bei ihren Projekten im Rahmen dieser Arbeit. Besonders KITA danke ich für die unglaubliche Unterstützung zum Ende dieser Arbeit!

Ich danke meinen Korrekturlesern FAlKo, Kita, Johannes, Markus, Stefan, IngO, JerRy und PETER für ihre Mühen meine wirren Gedankengänge zu entschlüsseln und mir beim Ordnen zu helfen. Ich hoffe die gröbsten Schnitzer sind ausgewetzt! Insbesondere FALKO und KITA, die den Großteil dieser Arbeit mehrfach unter die Lupe genommen haben, möchte ich ganz herzlich danken!

Bei dem gesamten Arbeitskreis bedanke ich mich für eine sehr schöne Zeit und die immer gute Atmosphäre!

Meiner Familie danke ich für die Unterstützung auf dem Weg hierhin. Da geht auch ein besonderer Dank an HANNA, JONATHAN und DANILO - die Göttinger Banausen. Es war schön einen Teil der Familie immer in der Nähe zu haben!

Zu guter Letzt möchte ich mich beim GGNB Office bedanken, das den, naja sagen wir mal nicht ganz reibungslosen Verlauf der Abgabe gut verkraftet hat. Das System funktioniert, das habe ich selbst getestet! 